\title{
Radiation Hard 3D Diamond Sensors for Vertex Detectors at HL-LHC
}

\author{
Dissertation
}

zur Erlangung des mathematisch-naturwissenschaftlichen Doktorgrades „Doctor rerum naturalium“

der Georg-August-Universität Göttingen

im Promotionsprogramm ProPhys

der Georg-August University School of Science (GAUSS)

\author{
vorgelegt von \\ Lars Graber \\ aus Helmstedt
}

Göttingen, 2015 
Betreuungsausschuss

Prof. Dr. Arnulf Quadt

PD Dr. Jörn Grosse-Knetter

Dr. Jens Weingarten

\section{Mitglieder der Prüfungskommission:}

Referent: Prof. Dr. Arnulf Quadt

II. Physikalisches Institut, Georg-August-Universität Göttingen

Korreferent: PD Dr. Markus Keil

CERN, Genf / Georg-August-Universität Göttingen

Weitere Mitglieder der Prüfungskommission:

PD Dr. Ralf Bernhard

II. Physikalisches Institut, Georg-August-Universität Göttingen

Prof. Dr. Wolfram Kollatschny

Institut für Astrophysik, Georg-August-Universität Göttingen

Prof. Dr. Tim Salditt

Institut für Röntgenphysik, Georg-August-Universität Göttingen

Jun.-Prof. Dr. Steffen Schumann

II. Physikalisches Institut, Georg-August-Universität Göttingen

Tag der mündlichen Prüfung: 21.01.2016

Referenz: II.Physik-UniGö-Diss-2015/04 


\begin{abstract}
Diamond is a good candidate to replace silicon as sensor material in the innermost layer of a tracking detector at HL-LHC, due to its high radiation tolerance. After particle fluences of $10^{16}$ protons $/ \mathrm{cm}^{2}$, diamond sensors are expected to achieve a higher signal to noise ratio than silicon. In order to use low grade polycrystalline diamonds as sensors, electrodes inside the diamond bulk, so called 3D electrodes, are produced. Typically, this kind of diamond material has a lower charge collection distance (CCD) than higher grade diamond, which results in a decreased signal amplitude. With 3D electrodes it is possible to achieve full charge collection even in samples with low CCDs by decoupling the spacing of the electrodes from the thickness of the diamond bulk. The electrodes are produced using a femtosecond laser, which changes the phase of the diamond material. The phase changed material is conductive and identified as nanocrystalline graphite using Raman spectroscopy. Due to a crater like structure of the channels on one side of the diamond, contacting of the channels is difficult. With the femtosecond laser setup conductive channels are produced at a laser power of $150 \mathrm{~mW}$ and a velocity of the diamond sample of $2-10 \mathrm{~mm} / \mathrm{s}$.
\end{abstract}





\section{Zusammenfassung}

Diamant ist aufgrund seiner hohen Toleranz gegenüber Strahlungsschäden ein aussichtsreicher Kandidat, um Silizium als Sensormaterial für die innerste Lage eines Spurdetektors am HL-LHC zu ersetzen. Nach Teilchenfluenzen von $10^{16}$ Protonen $/ \mathrm{cm}^{2}$ wird erwartet, dass Sensoren aus Diamant ein besseres Signal zu Rausch Verhältnis aufweisen, als Sensoren aus Silizium. Um günstigeren polykristallinen Diamanten als Sensor nutzen zu können, werden Elektroden im Diamanten, so genannte 3D Elektroden, produziert. Im Vergleich zu teureren

Diamantmaterialien, hat polykristalliner Diamant eine geringere „charge collection distance" (CCD). Dies führt zu einer geringeren Amplitude des Signals. Mit 3D Elektroden ist es möglich auch in Proben mit kleiner CCD volle Ladungssammlung zu erreichen, da der Abstand der Elektroden voneinander nicht mehr durch die Dicke des Sensors festgelegt ist. Die Elektroden werden mittels eines Femtosekundenlasers erzeugt, welcher eine Phasenumwandlung des Diamantmaterials hervorruft. Das phasenveränderte Material ist elektrisch leitend und wird mittels Ramanspektroskopie als nanokristalliner Diamant identifiziert. Durch eine

Kraterstruktur der Elektroden auf einer Seite des Diamanten ist deren Kontaktierung anspruchsvoll. Mit einer Leistung des Femtosekundenlasers von $150 \mathrm{~mW}$ und einer Geschwindigkeit des Diamanten von $2-10 \mathrm{~mm} / \mathrm{s}$ relativ zum Laserstrahl werden leitende Kanäle erzeugt. 

1. Introduction 1

2. Motivation 3

2.1. The Standard Model of Particle Physics . . . . . . . . . . . . . . . . 3

2.2. Physics beyond the Standard Model . . . . . . . . . . . . . . . . 5

2.3. Physics Implications on the Future of LHC . . . . . . . . . . . . 9

3. LHC \& ATLAS 11

3.1. The LHC Accelerator Complex . . . . . . . . . . . . . . . . . . . 11

3.2. The ATLAS Detector . . . . . . . . . . . . . . . . 14

3.3. Upgrade Plans for LHC and ATLAS . . . . . . . . . . . . . . . . 23

4. Silicon Pixel Detectors 25

4.1. Energy Loss of Particles in Matter . . . . . . . . . . . . . . . . 25

4.2. Signal Generation and Detection in Silicon . . . . . . . . . . . . . . 30

4.3. The ATLAS Pixel Detector . . . . . . . . . . . . . . . . 33

5. Diamond Pixel Detectors 39

5.1. Diamond Properties . . . . . . . . . . . . . . . . . . . 39

5.2. Production of artificial Diamonds . . . . . . . . . . . . . . . 43

5.3. Radiation Damage and Radiation Tolerance . . . . . . . . . . . . . . . 45

5.4. Pixel Electrode Geometries . . . . . . . . . . . . . . . . . . . . . 48

5.5. Production of Electrodes inside the Diamond Bulk . . . . . . . . . . . . 51

5.6. Metallisation of Diamonds . . . . . . . . . . . . . . . . . 56

6. 3D Electrodes in optical grade pCVD Diamond 59

6.1. Optical grade polycrystalline Diamond Samples . . . . . . . . . . . . . . 59

6.2. Femtosecond Laser Setup . . . . . . . . . . . . . . . . . . . . 61

6.3. Observation of phase changed Material . . . . . . . . . . . . . . . 62 
6.4. Measurement of channel induced Mechanical Stress . . . . . . . . . . . . 65

6.5. 3D Writing in Diamond . . . . . . . . . . . . . . . . . . 69

6.6. Raman Spectroscopy . . . . . . . . . . . . . . . . . . . 75

7. 3D Electrodes in detector grade pCVD Diamond $\mathbf{8 5}$

7.1. Detector grade polycrystalline Diamond Sample . . . . . . . . . . . . 85

7.2. Produced 3D Electrodes . . . . . . . . . . . . . . . . . 85

7.3. Raman Spectroscopy . . . . . . . . . . . . . . . . . . . . . . 89

7.4. Atomic Force Microscopy . . . . . . . . . . . . . . . . . . . . 100

7.5. Resistivity Measurements . . . . . . . . . . . . . . . . 105

$\begin{array}{ll}\text { 8. Conclusion \& Outlook } & 111\end{array}$

$\begin{array}{lr}\text { Bibliography } & 115\end{array}$

$\begin{array}{ll}\text { Appendices } & 123\end{array}$

A. Photos of Phase Change in pCVD Diamond $\quad 125$

A.1. Goe-pCVD-01 . . . . . . . . . . . . . . . . . . . . 125

A.2. Goe-pCVD-02 . . . . . . . . . . . . . . . . . . . . . 128

A.3. Goe-pCVD-03 . . . . . . . . . . . . . . . . . . . . . . 129

$\begin{array}{ll}\text { B. Raman Spectra of Phase Changed Material } & 131\end{array}$

B.1. Batch 2 of the detector grade pCVD Diamond Sample . . . . . . . . . . 131

B.2. Batch 3 of the detector grade pCVD Diamond Sample . . . . . . . . . 138 
CHAPTER 1

Introduction

The Standard Model of particle physics describes the composition of the known matter and its fundamental interactions on a particle scale. In the past decades it has performed extremely well. It contains all known particles, including gauge bosons for three of the four fundamental forces. With the discovery of the Higgs boson in 2012 by ATLAS and CMS at the Large Hadron Collider (LHC), all particles of the Standard Model have been discovered.

Still, predictions made by the Standard Model are further evaluated. Deviations from the expected values could give a hint towards physics beyond the Standard Model. For example, this can include candidates for Dark Matter and a gauge boson for the gravitational force. Since no deviations from the Standard Model have been found yet, except the mass of the neutrinos, new levels of precision in the measurement of these values have to be achieved. This means that more statistics has to be acquired and new energy regimes have to be explored. Since upgrading the energy of an accelerator or building a new one is a time consuming and expensive task, higher statistics are easier to achieve. In order to perform the data acquisition in a meaningful time frame, the luminosity of the accelerator has to be increased. Accordingly, an upgrade of the LHC, the High Luminosity Large Hadron Collider (HL-LHC) is planned for 2024.

Higher luminosity causes increased particle flux in the detectors, which in turn increases the radiation damage in the detector components. These components have to be radiation tolerant enough to withstand these levels of radiation for several years, as they cannot be exchanged easily. The innermost layer of the vertex detector system, which is only few centimetres away from the colliding beams, suffers most from the radiation damage. For this harsh environment, diamond sensors are a candidate, as they are more radiation tolerant than silicon sensors, which are used so far. 


\section{Introduction}

In order to successfully operate diamond as a sensor material, special properties of diamond have to be addressed, especially the trapping of charges. The effects of this trapping can be reduced by using $3 \mathrm{D}$ electrodes, which expand through the bulk of the sensor. In this thesis, the fabrication and evaluation of such 3D electrodes in a diamond sensor will be described.

First, in chapter 2 the need for the HL-LHC and accordingly radiation tolerant sensors is motivated. The current LHC and the ATLAS detector are presented in chapter 3, as well as the upgrade plans for both. In order to be qualified as a sensor material for the innermost layer of a future tracking detector, diamond has to outperform the sensor material currently used for vertex detectors, which is silicon. Therefore, the characteristics of silicon sensors are discussed in chapter 4 and the current Pixel Detector of the ATLAS experiment is described. Chapter 5 presents the properties of diamond sensors and their manufacture. Their radiation hardness is discussed, as well as the theoretical background of the production of 3D electrodes in the diamond bulk. Such 3D electrodes in optical grade polycrystalline diamonds and the measurements performed to characterise their properties are presented in chapter 6 . In chapter 7 , the focus is placed on the determination of the material of the electrodes and their structures on the diamond surface. The influence of these two properties on the conductivity of the channels is evaluated. Finally, a summary of the thesis and an outlook towards the production of a full detector layer is given in chapter 8 . 
CHAPTER 2

Motivation

This chapter gives an overview of the basic principles and aims of the scientific measurements at the LHC. First, the Standard Model (SM) of particle physics is described in section 2.1. Searches for new particles and their impact on the Standard Model are presented in section 2.2. Section 2.3 discusses the impact of these searches for the future of LHC.

\subsection{The Standard Model of Particle Physics}

It seemed like the picture of the subatomic particles was complete, after the discovery of the neutron by J. Chadwick in 1932 [1]. All matter around us seemed to consist of atoms which themselves can contain three fundamental particles. These were namely electrons, protons and neutrons.

However, subatomic forces could not be explained and cosmic ray experiments showed that more particles exist. As a result of these experiments and measurements at accelerators, dozens of new particles were discovered until the end of the 1960s. These did not fit into the picture of only three fundamental particles. In 1964 M. Gell-Mann and G. Zweig proposed the concept of quarks [2,3]. Their idea was that the proton and neutron are in fact not fundamental but are composites of other fundamental particles, the so called quarks. Initially, they proposed three quarks namely the up, down and strange quark. With this concept, which lead to the Standard Model of particle physics, it was possible to order the discovered particles and even predict new ones. The amount of quarks was later expanded with the observation of the charm, bottom and top quark [4-8].

Additionally, the Standard Model also includes six leptons. Three of them carry a negative electric charge, which are the electron, muon and tau. The remaining three 


\section{Motivation}

\begin{tabular}{l|rrrr}
\hline Particle & Mass & Electric charge & Weak isospin & Colour charge \\
\hline \multicolumn{4}{c}{ Leptons } \\
\hline Electron & $511 \mathrm{keV}$ & -1 & $-1 / 2$ & none \\
Electron neutrino & $<2 \mathrm{eV}$ & 0 & $1 / 2$ & none \\
\hline Muon & $106 \mathrm{MeV}$ & -1 & $-1 / 2$ & none \\
Muon neutrino & $<0.19 \mathrm{MeV}$ & 0 & $1 / 2$ & none \\
\hline Tau & $1.78 \mathrm{GeV}$ & -1 & $-1 / 2$ & none \\
Tau neutrino & $<18.2 \mathrm{MeV}$ & 0 & $1 / 2$ & none \\
\hline & & Quarks & & \\
\hline Down & $4.8 \mathrm{MeV}$ & $-1 / 3$ & $-1 / 2$ & yes \\
Up & $2.3 \mathrm{MeV}$ & $2 / 3$ & $1 / 2$ & yes \\
\hline Strange & $95 \mathrm{MeV}$ & $-1 / 3$ & $-1 / 2$ & yes \\
Charm & $1.28 \mathrm{GeV}$ & $2 / 3$ & $1 / 2$ & yes \\
\hline Bottom & $4.18 \mathrm{GeV}$ & $-1 / 3$ & $-1 / 2$ & yes \\
Top & $173 \mathrm{GeV}$ & $2 / 3$ & $1 / 2$ & yes \\
\hline
\end{tabular}

Table 2.1.: Properties of the fermions of the Standard Model [10].

leptons are the corresponding neutrinos, one for each charged lepton. They have no electric charge and in the Standard Model no mass. Measurements of the oscillation of the neutrino flavour however require them to have a small mass [9]. Today, only upper limits of their masses are known [10].

The leptons and quarks are grouped into three generations, of which only particles of the first one are stable. The particles of the higher generations have the same characteristics as their corresponding first generation partner, but they have a higher mass ${ }^{1}$ and can therefore decay into lighter particles. This results in a finite lifetime. Quarks and leptons all have a spin of $1 / 2$, therefore they are fermions. For each fermion there exists a corresponding anti-particle, which has the same properties, but different quantum numbers for the charges ${ }^{2}$. A summary of the properties of the fermions is given in table 2.1.

The Standard Model also describes the gauge bosons for three of the four forces in nature. A summary of the properties of the bosons is given in table 2.2. Only a possible particle mediating the gravitational force, which is not included in the Standard Model,

\footnotetext{
${ }^{1}$ As the mass hierarchy of the neutrinos has not been determined yet, this is not necessarily true for neutrinos.

${ }^{2}$ As neutrinos have no electric or colour charge, they could be their own anti-particle, so called Majorana neutrinos [11].
} 
is unknown. The gauge boson of the electro-magnetic force is the photon. It couples to the electric charge of particles. This means that it couples to all fermions except for the neutrinos. As the photon itself does not have an electric charge, it does not couple to itself. It has a mass of $m_{\gamma}=0 \mathrm{eV}$ and propagates with the speed of light.

Only quarks and gluons are affected by the strong force. These particles have a property called colour charge. There are three colours (typically red, green \& blue) and three anti-colours. The gauge bosons of the strong force are the gluons, which like the photon have a mass of zero. The gluons do themselves carry a colour charge and therefore do interact with themselves.

In contrast to the gauge bosons of the other forces, the bosons of the weak force are massive. As they are heavy with $m_{W}=(80.39 \pm 0.02) \mathrm{MeV}$ and $m_{Z}=(91.188 \pm$ $0.002) \mathrm{MeV}$ for the $W^{ \pm}$and $Z$, respectively [10], the strength of the force is small compared to the strong and electro-magnetic force. These gauge bosons couple to every other particle, except gluons ${ }^{3}$.

The final piece of the Standard Model is the Higgs boson. Its coupling strength depends on the mass of the particles involved in the interaction. This means that it couples to all particles except photons and gluons ${ }^{4}$. After being predicted by F. Englert, R. Brout, P. W. Higgs and others in 1964 [12-14] it was finally discovered at the LHC in $2012[15,16]$. Since then its properties and couplings have been measured [17-21]. The current results show no significant deviation from the predicted theoretical particle of the Standard Model.

\subsection{Physics beyond the Standard Model}

With the discovery of the Higgs boson, all particles predicted by the Standard Model have been found. The predictions of the Standard Model on e.g. branching ratios have been remeasured numerous times and no significant deviation from theory prediction has been found, yet. But the Standard Model is incomplete in the sense that it cannot explain everything we are aware of. One major missing ingredient is the exclusion of the gravitational force. While it is highly suppressed due to its weakness on atomic scales, it is the dominant force on the macroscopic scale. The Standard Model does not include its potential gauge boson, the graviton.

Another issue which cannot be explained with the Standard Model is the rotational speed of galaxies. From the amount of mass visible in rotating galaxies, the rotation velocity should decrease with larger radii. This is not the case and the rotational speed stays almost constant for large radii [22]. The measurement indicates that there should

\footnotetext{
${ }^{3}$ As the $Z$ has no electric charge, it does not couple the photon.

${ }^{4}$ As the Higgs does not have a electric or colour charge, the photon and gluon do not couple to it either.
} 


\section{Motivation}

\begin{tabular}{l|crr}
\hline Particle & Mass & Electric charge & Colour charge \\
\hline \multicolumn{4}{c}{ Electro-magnetic force } \\
\hline Photon & none & 0 & none \\
\hline \multicolumn{4}{c}{ Weak force } \\
\hline $\mathrm{W}$ & $80.4 \mathrm{GeV}$ & 0 & none \\
$\mathrm{Z}$ & $91.2 \mathrm{GeV}$ & none \\
\hline \multicolumn{5}{c}{ Strong force } \\
\hline Gluon & none & 0 \\
\hline \multicolumn{5}{c}{ Higgs mechanism } \\
\hline Higgs & $\approx 126 \mathrm{GeV}$ & yes \\
\hline
\end{tabular}

Table 2.2.: Properties of the bosons of the Standard Model [10].

be more matter in the galaxy than visible. This matter is called dark matter, as it does not interact via the electro-magnetic force. Further evidence of the existence of such matter is obtained by measurements of gravitational lensing [23]. Objects with large masses like galaxies bend, according to Einstein, the space-time and therefore are able to deflect even light [24]. The deflection depends on the amount of mass of the lensing object. Comparing the mass needed for the measured deflection to the visible mass yields a discrepancy and hence indicates the presence of dark matter.

Although current measurements indicate that there is more than five times more dark matter than visible matter in the universe [25], the Standard Model does not contain a dark matter particle nor has this particle directly been measured. One theory for such a dark matter particle is the weakly interacting massive particle (WIMP). It postulates a particle which does neither carry an electro-magnetic nor a colour charge. Accordingly, it can only interact via the weak force and gravity. Such a particle should also be stable on cosmic scales, i.e. at least in the order of the age of the universe, as otherwise it would have decayed by now.

A candidate for such a WIMP is the lightest supersymmetric particle (LSP). The theory of supersymmetry (SUSY) in its general form adds to each fermion of the Standard Model a supersymmetric boson and to each boson a supersymmetric fermion. One common postulate in these theories is the conservation of R-parity in an interaction. The $\mathrm{R}$ value for Standard Model particles is 1 , for supersymmetric particles -1 . This means, that supersymmetric particles can only be produced pair-wise from Standard Model particles. Therefore in case of R-parity conservation the lightest supersymmetric particle has to be stable. This makes it a reasonable WIMP candidate. 


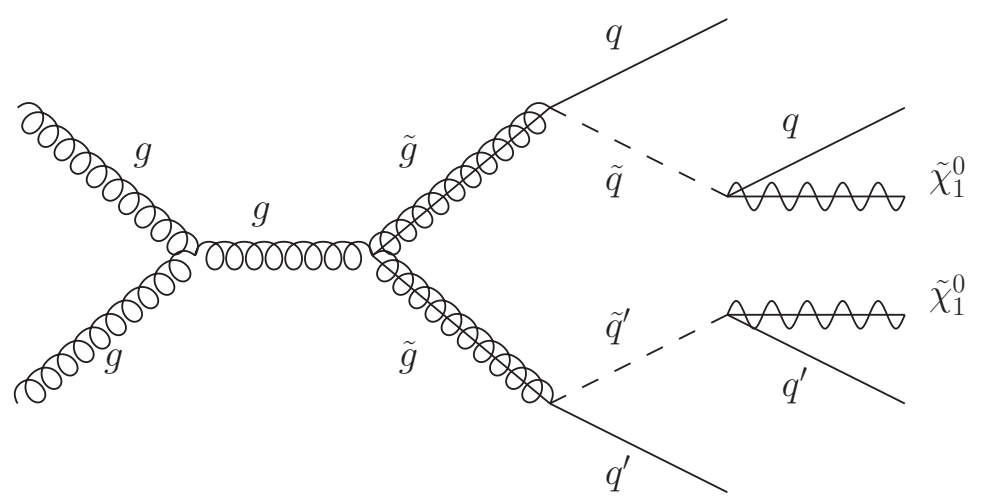

Figure 2.1.: Feynman diagram of a possible production of an LSP pair, which would escape the detector. Therefore, it can be detected by missing transverse energy. Initially, a gluino pair $(\tilde{g})$ is produced which decays via squarks $(\tilde{q})$ into the LSP $\left(\tilde{\chi}_{1}^{0}\right)$.

In case of unbroken SUSY, the supersymmetric partners and Standard Model particles would have the same mass, like particles and anti-particles do. Since no supersymmetric particles have been observed yet, this is not the case. Accordingly, if SUSY is realised, the supersymmetric particles have to be much heavier than their Standard Model counterparts. As the masses of the supersymmetric particles are now free parameters, an unconstrained SUSY model would have more than 100 free parameters [26]. These are far too many parameters to vary at once. Consequently, for promising benchmark models, the number of free parameters is reduced by putting constraints on some of these parameters. For example, additional $\mathrm{CP}$ violating phases are assumed to be zero and no flavour changing neutral currents are allowed. Such models are called minimal supersymmetric models (MSSM).

The cross section of the interaction of a WIMP with normal matter is very small, otherwise it would have already been detected. Direct searches for WIMPs often try to measure their recoil on a target. For such experiments, an ultra low background is required, which cannot realistically be achieved at a particle accelerator. Instead their production is investigated. A pair-wise produced WIMP would escape the detector undetected. This can be measured by the large amount of missing transverse energy in the detector. It is a similar signature to an event with neutrinos, as they do also escape the detector. But in this case the leptons corresponding to the neutrinos are missing. Consequently, events with only hadronic jets and a large amount of missing transverse energy are an indication of SUSY.

If not the LSP but another unstable SUSY particle is produced, this particle could decay inside of the detector. The signature therefore is a displaced vertex, i.e. a vertex seemingly not related to a primary interaction in the detector. The SUSY particle would 


\section{Motivation}

be produced in the primary interaction and propagate through the detector undetected. At some point it would decay into matter and the LSP. The LSP would still escape the detector, but the matter could be detected. An example of such an event is given in figure 2.1 .

SUSY does not only contain a candidate for dark matter but also could possibly solve other problems of the Standard Model of particle physics. One pending issue is the unification of the three forces of the Standard Model at the Planck scale, which starts at particle energies of $10^{19} \mathrm{GeV}$. A reasonable assumption is that, in that regime, the three forces would unify into one general force. For this, the value of their coupling constants has to be equal at some point. The evolution of the strength of the coupling constants was measured at the electroweak scale and theoretically expanded to the Planck scale. Extrapolating from measurements according to the Standard Model, they converge, but do not meet at the exact same point. Applying certain SUSY models, this can be corrected and they meet at the same point, indeed.

If SUSY cannot be measured at the available centre of mass energy at the LHC, there still is the possibility of determining its influence by indirect measurements. One channel which could be sensitive to new physics is the associated production of a Higgs and a top-antitop quark pair. Since the mass of the top is the largest of any SM particle (see table 2.1) the Yukawa coupling of the Higgs to the top quark is larger than to any other particle. In fact, it is expected to be close to unity. Any deviation from the expected value would give hints to other particles with an even larger mass than the mass of the top quark.

A measurement of the coupling strength in the decay of a Higgs boson to a topantitop quark pair is not possible, as this decay is kinematically forbidden. Accordingly, in order to measure this coupling, the Higgs has to be produced in association with the top-antitop quark pair, which is kinematically possible at the centre of mass energies of the current LHC. However, compared to other production modes of the Higgs, its cross section is highly suppressed, see figure 2.2. This means that in order to measure the associated production the background has to be understood very well and a lot of integrated luminosity has to be recorded. This has been done with Run I data (e.g. see reference [27]) and no deviation from the SM prediction was measured.

However, there are other processes like e.g. a top quark decaying into a Higgs and any up-type quark [29]. Such a flavour changing neutral current is highly suppressed in the SM. But it could be allowed in models like SUSY. Still, the branching ratio would be very tiny, so that it might be impossible to measure precisely even with the full data set of Run II data. 


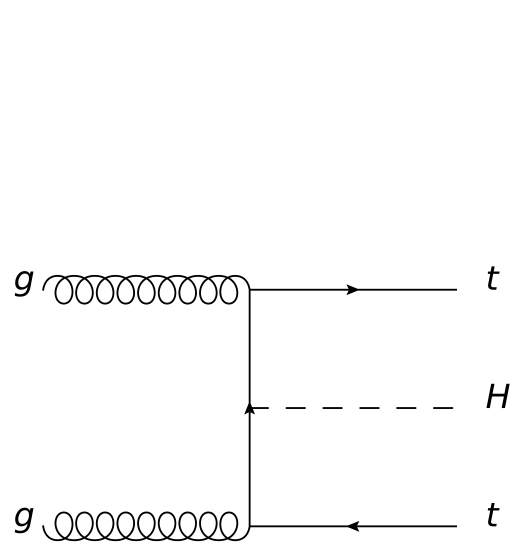

(a)

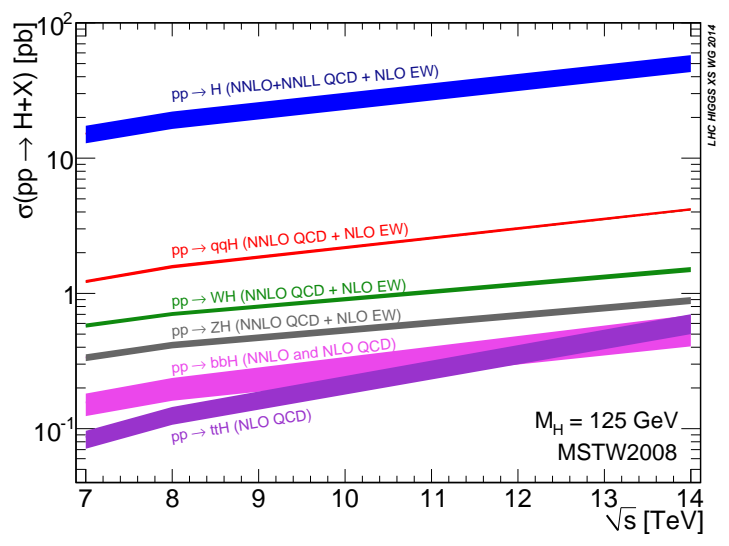

(b)

Figure 2.2.: One of the possible production modes for a Higgs boson in association with a top-antitop pair (a). Cross sections for different production modes of the Higgs boson [28]. The associated production with a top-antitop pair is small compared to almost all other modes (b).

\subsection{Physics Implications on the Future of LHC}

As motivated above, the LHC with Run II offers a wide range of discovering new physics, by measuring it either directly or indirectly. Furthermore, the properties of the current content of the SM, especially the Higgs, can be measured to a new level of precision.

Should no new physics, like e.g. SUSY particles, become directly accessible at the LHC and the couplings are too small to measure their influence even with the full Run II data set, there are two concepts to proceed with the LHC. The first one is by increasing the centre of mass energy. This is not easily feasible, since new dipole magnets with a higher magnetic field are needed to keep the protons on track. As the dipole magnets do make up a good share of the LHC, this basically means building a whole new accelerator. Obviously, this is very expensive and time consuming.

The second alternative is to keep the current centre of mass energy but increase the instantaneous luminosity. This scenario is called High-Luminosity LHC (HL-LHC). As shown in Section 2.2 the measurements of new physics is possible, by measuring the influence of new physics on SM processes. However, for these measurements, a huge number of recorded events is needed. The advantage of just increasing the instantaneous luminosity is that the accelerator itself can nearly be kept as it is. In order to increase the luminosity, the easiest way is to increase the number of particles which collide. The only two major upgrades needed in this case are the cavities which accelerate the particles and the pre-accelerator complex (more details on this in Section 3.3). Both have to be enabled to handle many more particles than for the current LHC operation. 


\section{Motivation}

While in this scenario the accelerator complex is only mildly modified, the detectors face much harder challenges. The increase of instantaneous luminosity also increases the particle flux in the detector. This leads to higher doses of irradiation the detectors have to withstand. For example for the innermost tracking layer, it increases from $2.7 \times 10^{14} \mathrm{n}_{\mathrm{eq}} \mathrm{cm}^{-2}$ in Run I to $1.4 \times 10^{16} \mathrm{n}_{\mathrm{eq}} \mathrm{cm}^{-2}$ for HL-LHC [30,31]. As there are more primary interactions to achieve a higher luminosity, the whole tracking system should also be able to resolve the huge number of tracks. In order to achieve these goals, new sensor concepts and materials have to be investigated. 
CHAPTER 3

\section{LHC \& ATLAS}

The Large Hadron Collider (LHC) is a proton-proton accelerator located at the European Organization for Nuclear Research (CERN) in Geneva, Switzerland. It is currently the most powerful man-made particle accelerator with a nominal centre of mass energy for proton collisions of $14 \mathrm{TeV}$. It hosts four major particle detectors, namely ALICE [32], ATLAS [30], CMS [33] and LHCb [34]. A detailed description of the accelerator and its performance in the past years is given in section 3.1. The work presented in this thesis is geared towards an upgrade of the ATLAS detector, hence this detector is described in section 3.2. Plans for the future upgrade for the LHC as well as for ATLAS are presented in section 3.3 .

\subsection{The LHC Accelerator Complex}

The LHC [35] is a circular particle accelerator, which is about $27 \mathrm{~km}$ long and located in a tunnel between the Lake Geneva and the Jura mountains, straddling the border between Switzerland and France. It is designed to accelerate protons to a nominal final energy of $7 \mathrm{TeV}$ per beam. This yields a centre of mass energy of $14 \mathrm{TeV}$. The acceleration of ions is also possible, as well as a mixed mode with one beam consisting of protons and one of ions.

The LHC is not able to accelerate these particles from zero to the final energy, but it can only handle protons with a minimal energy of $450 \mathrm{GeV}$. Therefore, a chain of preaccelerators is needed, which is shown in figure 3.1. Hydrogen atoms are ionised and accelerated to $50 \mathrm{MeV}$ at the Linac II. Subsequently, they reach the Booster to be accelerated to $1.4 \mathrm{GeV}$ and are then injected into the Proton Synchrotron (PS) where they reach $25 \mathrm{GeV}$. The last stage is the Super Proton Synchrotron (SPS) to accelerate the particles to the minimal LHC energy. The LHC can also accelerate heavy ions, for 


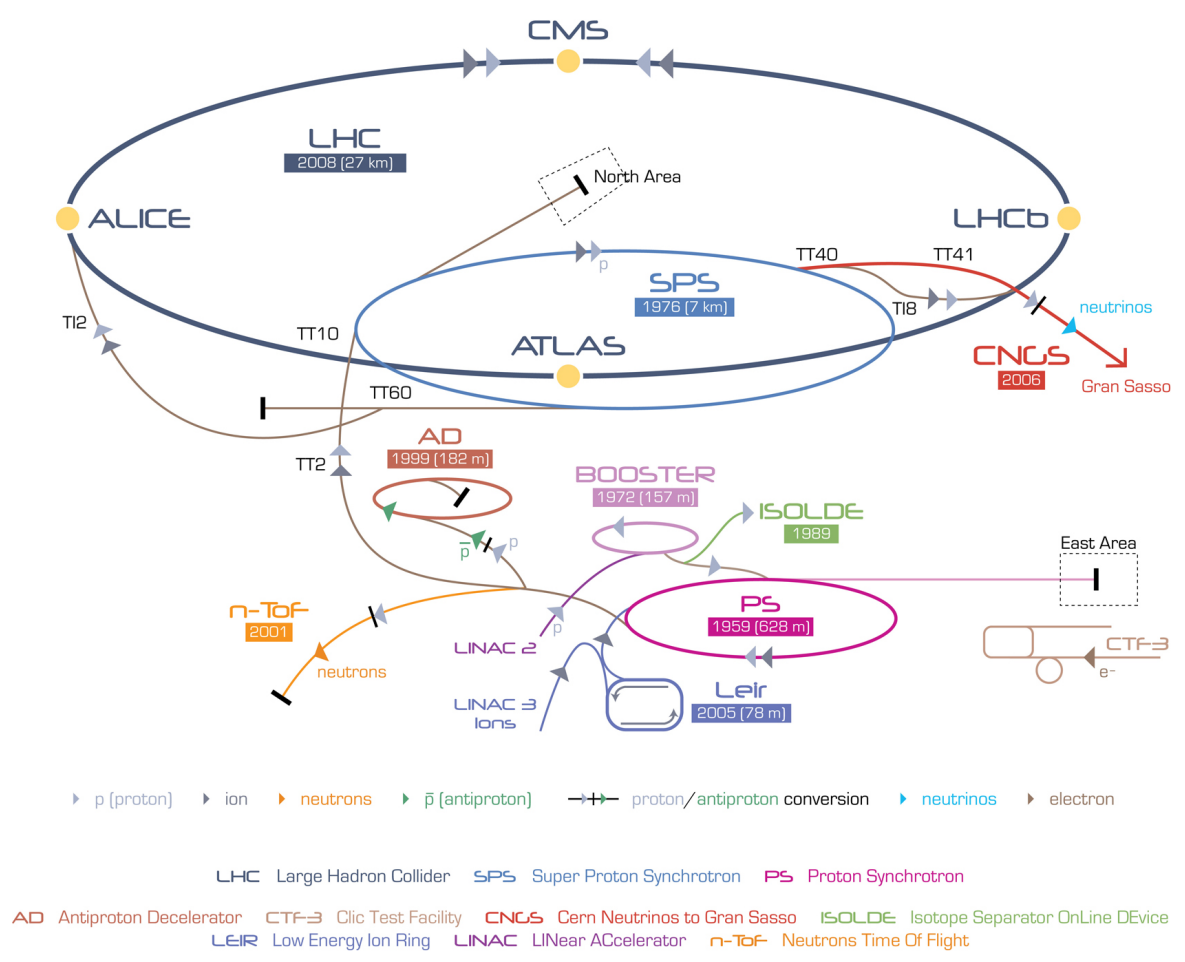

Figure 3.1.: CERN accelerator complex. The LHC and its preaccelerators are illustrated.

which only the start of the cascade is different, since they are first accelerated in the Linac III and the Low Energy Ion Ring (LEIR) before reaching the PS.

The largest share of the circumference of the LHC consists of the 1232 dipole magnets. Each of them is $14.3 \mathrm{~m}$ long and provides a magnetic field with a strength of $8.33 \mathrm{~T}$ [35]. The magnetic field is orientated perpendicular to the beam, so that it can bend it and keep it on track. A schematic cut through such an dipole magnet is shown in figure 3.2. In order to achieve a field strength of $8.33 \mathrm{~T}$, the magnets are superconducting. The utilised material is niobium-titanium which is cooled to $1.9 \mathrm{~K}$ using supra fluid helium. In order to reduce material and cooling costs, the dipoles are designed in such a way that both beam pipes fit into the same cryostat. This is very challenging, as the beam pipes are so close together that they are coupled mechanically, as well as magnetically.

Almost all of the remaining length of the LHC is instrumented with magnets of higher order, such as quadrupoles. These are used to focus the beam. The acceleration is achieved by eight cavities per beam, located at a single point at the LHC. The usage of cavities also prevents a continuous beam structure. Instead, the particles are separated in so called bunches with each bunch containing up to $1.15 \times 10^{11}$ protons. When the LHC is fully filled, it can handle 2808 bunches per beam with a spacing of $25 \mathrm{~ns}$. 


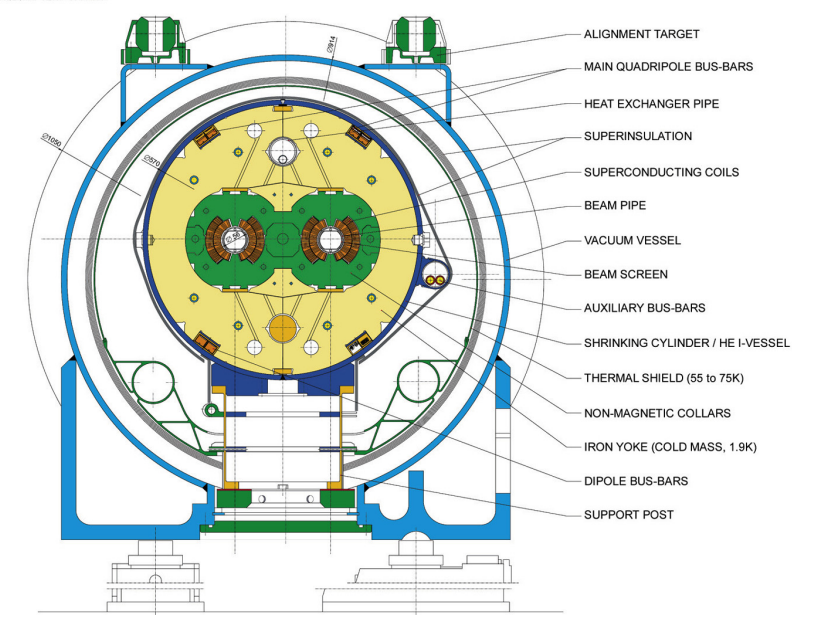

Figure 3.2.: Schematic cut through a dipole magnet of the LHC [35]. The most challenging part of the design is the close proximity of the two beam pipes.

These bunches can collide at four points of the LHC, where the four major experiments are located. Two of these experiments, ATLAS and CMS, are multi-purpose detectors. This means, that they are searching for a wide variety of processes like e.g. the Higgs boson, dark matter or SUSY particles. The detector concept of those two experiments differ so that the experiments can verify each others results. A detailed description of the ATLAS detector can be found in section 3.2. The third experiment is the LHCb detector, which in contrast to the other experiments, has no full coverage of the collision events, but is built asymmetrically in forward direction. Its focus is on B-physics, especially on CP-violation. The fourth large experiment ALICE has its main focus on ion-ion collisions. It aims to measure the properties of quark-gluon plasma.

Two main parameters characterise an accelerator, the energy of the accelerated particles and the luminosity. The luminosity $L$ is the number of particle crossings per time and area, defined as

$$
L=\frac{n \cdot N_{1} \cdot N_{2} \cdot f}{A} .
$$

Here $n$ is the number of colliding bunches, $N_{i}$ is the number of particles in each bunch, $f$ is the revolution frequency of a single bunch in the synchrotron and $A$ the area of the overlapping bunches. In order to gather as much data as possible, the luminosity should be high. For the LHC the design luminosity is $L=10^{34} \mathrm{~cm}^{-2} \mathrm{~s}^{-1}$, which is the world record for hadron colliders. To quantify the amount of total collected data, the luminosity is integrated over time, the so called integrated luminosity.

After a successful start in September 2008, after a few days of operation, unfortunatley an incident due to a failure in a dipole magnet occured. A solder connection 


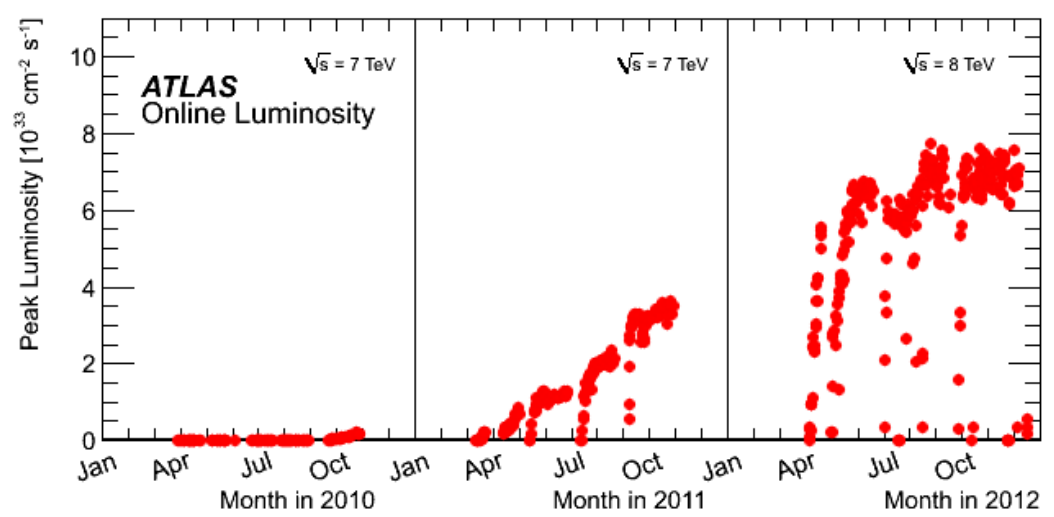

Figure 3.3.: Evolution of the luminosity delivered by the LHC to ATLAS during Run I [36].

in one dipole magnet was faulty and caused the superconducting magnet to heat up. This quench resulted in heavy damage in that section and a shut down of the LHC for one year. During that year, all solder connections were checked. In order to ensure a save operation, it was decided to operate the LHC below its design values, before fully exploiting its power. The operation started again in November 2009 and data taking at a centre of mass energy of $7 \mathrm{TeV}$ was performed during 2010. From 2011 until the beginning of 2013 the LHC took data at a centre of mass energy of $8 \mathrm{TeV}$. This whole period of data taking is referred to as "Run I". The evolution of the luminosity during Run I is shown in figure 3.3, the integrated luminosity in figure 3.4.

From the beginning of 2013 the magnets of the LHC were checked and upgraded to allow operation with design parameters. The experiments also upgraded parts of their detectors. For example ATLAS inserted a new fourth layer into the Pixel detector, the Insertable B-Layer (IBL). Since spring 2015 the LHC operates at a centre of mass energy of $13 \mathrm{TeV}$. For future plans of the LHC see section 3.3.

\subsection{The ATLAS Detector}

ATLAS [30] is one of the main experiments at the LHC and is located at one interaction point (IP) of the LHC. It nearly fills the complete underground cavern it is built in with physical dimensions of $44 \mathrm{~m}$ length and $25 \mathrm{~m}$ width and height. The total weight of the detector is about $7000 \mathrm{t}$. It is intended as a multi purpose detector and covers almost the full solid angle. A schematic view of the detector is shown in figure 3.5. The detector is mainly separated into three parts, each with a different focus on the quantities which can be measured. These three parts are the tracking system, the calorimeters and the muon system. Most of these systems are further divided into layers of different detector types. The tracking system, also called Inner Detector (ID), is enclosed in a solenoid 


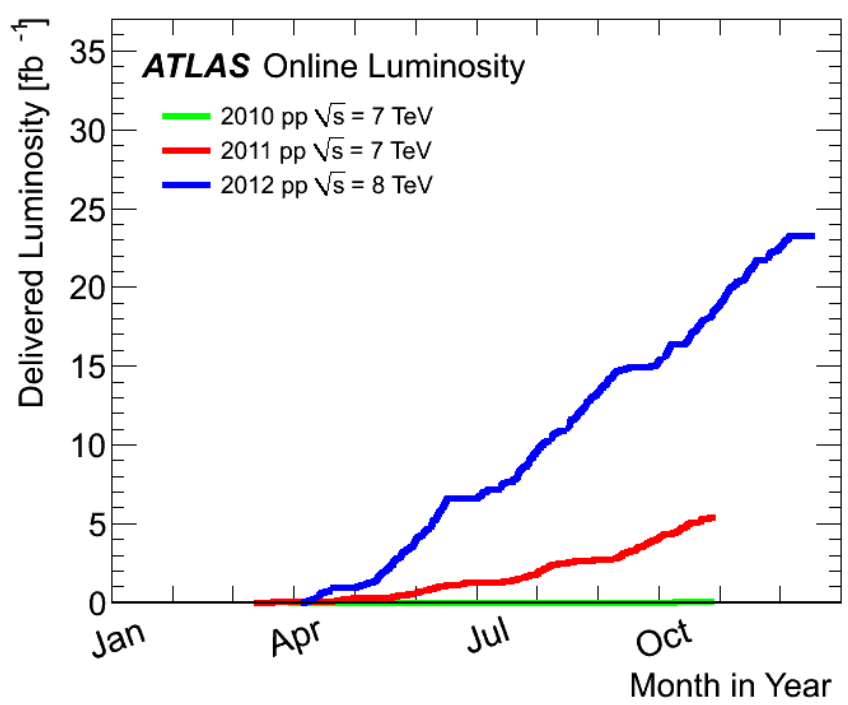

Figure 3.4.: Evolution of the integrated luminosity delivered by the LHC to ATLAS during Run I [36].

magnet generating a magnetic field with a strength of $2 \mathrm{~T}$ parallel to the beam. The outermost system, the muon system, has a toroidal magnetic field.

ATLAS uses a cylindrical coordinate system. The z-axis is oriented along the beam pipe with $z=0$ located in the centre of the detector. The azimuthal angle $\phi$ is measured around the beam pipe, with $\phi=0^{\circ}$ pointing towards the centre of the LHC ring. The polar angle $\theta$ is measured from the beam axis. In particle physics this angle is typically not used but instead the pseudo rapidity $\eta$, defined as $\eta=-\ln \tan (\theta / 2)$, is quoted. The advantage is that differences in the pseudo rapidity are Lorentz-invariant, whereas differences in the polar angle are not. Additionally, the radial distance $R$ from the beam pipe is used.

The different subcomponents are described below, except the innermost detector, the Pixel Detector. As this thesis is about a sensor candidate for an upgrade for this component, this detector is described in more detail in chapter 4.3. Unless indicated otherwise, the numerical values in the following sections are taken from [30].

\subsubsection{The Inner Detector}

The Inner Detector (ID) focuses on tracking the particles from the hard interactions. A schematic view is shown in figure 3.6. It has dimensions of about $6.2 \mathrm{~m}$ in length and $2.1 \mathrm{~m}$ in diameter. The ID consists of three sub detectors. The innermost detector, which has to cope with the highest particle flux, is the Pixel Detector. This part has, due to the pixel geometry of the read out electrodes, the highest granularity of the whole 


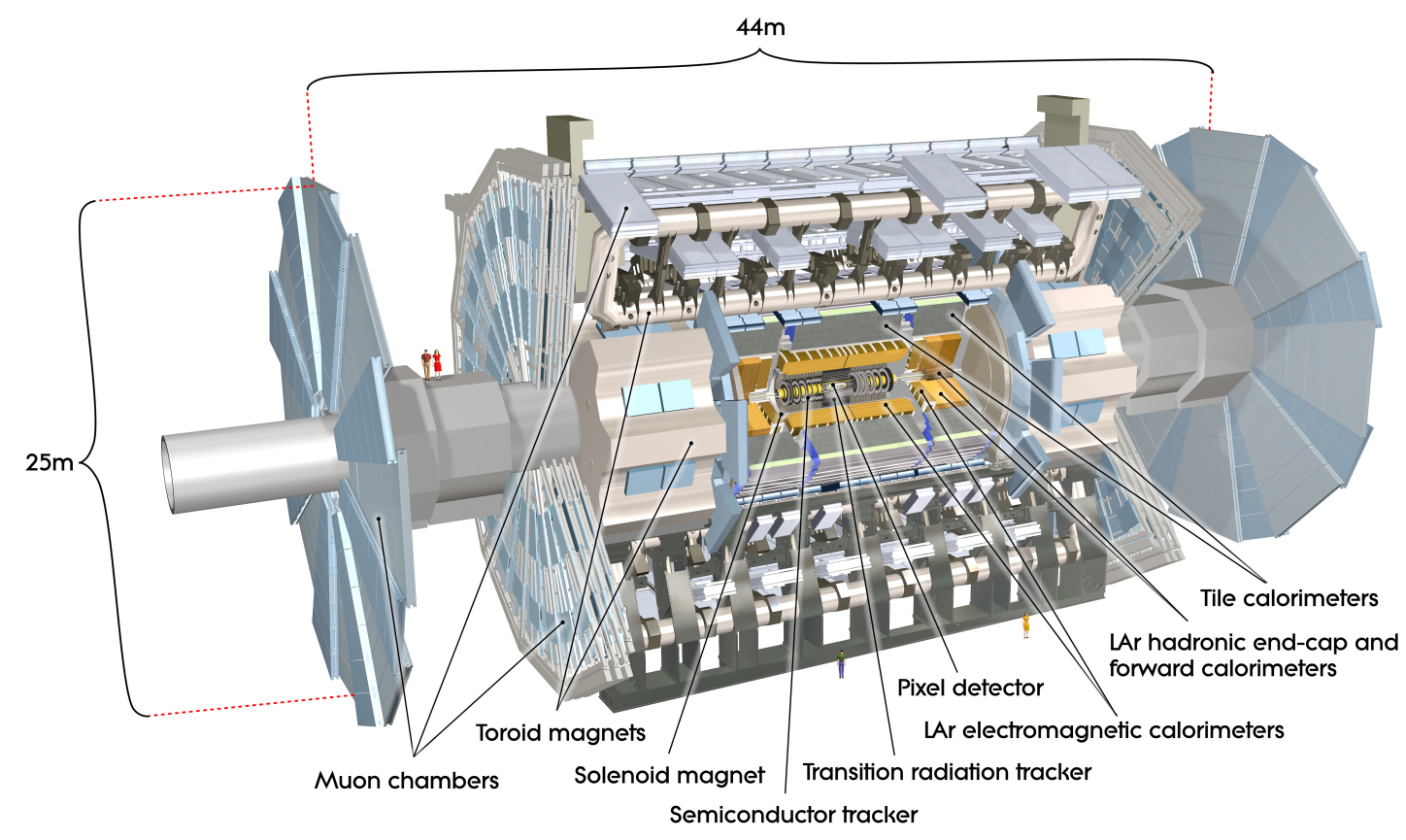

Figure 3.5.: Schematic view of the ATLAS detector [30].

ATLAS detector. The next system is the SemiConductor Tracker (SCT) which uses strip electrodes. The outermost part is the Transition Radiation Tracker (TRT).

\section{The Semiconductor Tracker}

The SemiConductor Tracker (SCT) uses silicon as sensor material. In this case the electrodes are strips. Compared to a pixel electrode, a single strip electrode yields one dimensional tracking information only. Combining two layers of strip electrodes which are slightly tilted with respect to each other, it is possible to retrieve two dimensional tracking information. The position of the particle can be reconstructed at the intersection of the strips, which have been hit. This works as long as just a few particles hit a module, so that no ambiguities for the combination are possible. The advantage of the strip geometry compared to pixel geometry is a reduction in read out channels and thus in material budget for the read out electronics, cables etc. The modules are also considerably cheaper to produce compared to pixel modules.

The SCT consists of 4,088 modules organised in four barrel layers and nine end cap layers on each side. Each of the barrel layers extends $149.8 \mathrm{~cm}$ along the beam pipe. They are mounted at radii of $29.9 \mathrm{~cm}, 37.1 \mathrm{~cm}, 44.3 \mathrm{~cm}$ and $51.4 \mathrm{~cm}$ around the beam pipe. The end cap discs are located between $|z|=85.4 \mathrm{~cm}$ and $|z|=272 \mathrm{~cm}$. Overall the SCT covers a range up to $|\eta|<2.5$ with at least four SCT hits in that range. 


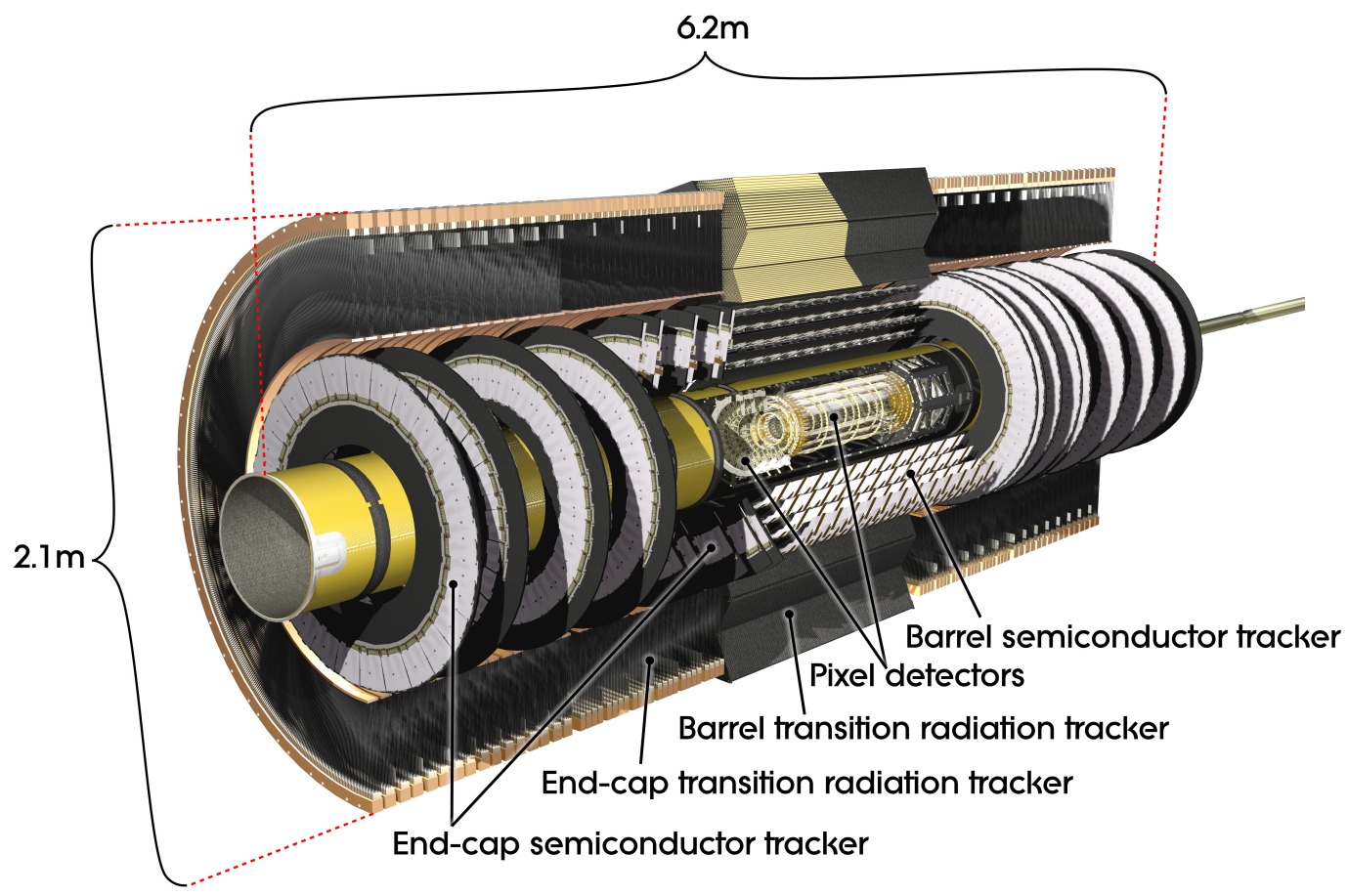

Figure 3.6.: Schematic view of the ATLAS Inner Detector [30]. It consists of, from inner to outer, the Pixel Detector, the Silicon Strip Detector and the Transition Radiation Tracker. In this picture the new innermost layer of the Pixel Detector, the IBL, is missing which was inserted for Run II.

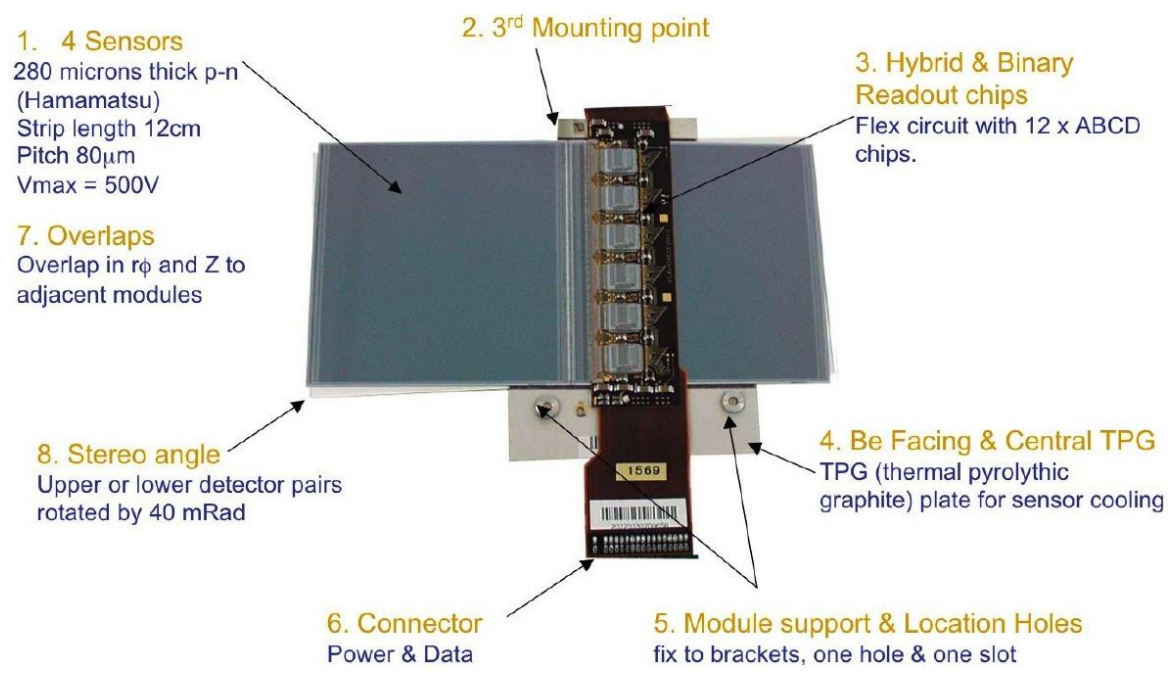

Figure 3.7.: An SCT barrel module [37]. 
The barrel modules consist of two layers of $285 \pm 15 \mu \mathrm{m}$ thick silicon sensors with 768 active strips with a pitch of $80 \mu \mathrm{m}$. Each layer itself consists of two sensors with approximately $6 \mathrm{~cm}$ long strips, which are daisy chained. The two layers are slightly tilted against each other with $40 \mathrm{mrad}$. A picture of such a module is shown in figure 3.7. To cope with the disc structure of the end caps, the strips on these modules are not any more oriented in parallel in one layer on the sensor, but have a slight tilt of $161.5-207.0 \mu \mathrm{rad}$. The sensors are designed to withstand a radiation dose of $3 \times 10^{14} \mathrm{n}_{\mathrm{eq}} \mathrm{cm}^{-2}$.

\section{The Transition Radiation Tracker}

The Transition Radiation Tracker (TRT) consists of gasfilled straw tubes, which are interleaved with a matrix of polypropylene fibres. These fibers serve as transition radiation material. Transition radiation occurs, when a relativistic particle crosses an interface between two materials with different dielectric constants. The tubes have a diameter of $4 \mathrm{~mm}$ and a length of $144 \mathrm{~cm}$ and $37 \mathrm{~cm}$ for barrel and end cap region, respectively. They are filled with a Xenon based gas mixture at $5-10$ mbar overpressure. In the centre of each tube is a wire with a diameter of $31 \mu \mathrm{m}$ made mainly out of tungsten. The straw is operated at $-1530 \mathrm{~V}$ to achieve an amplification of $2.5 \times 10^{4}$ and read out at the end of each tube.

In total, the TRT consists of approximately 351,000 tubes organised in 73 layers in the barrel and 160 layers in the end cap region. These yield a coverage of up to $|\eta|<2$. Compared to the other two components of the ID, the TRT resolves the tracks in $R-\phi$ only. This is enough to enhance the tracking pattern recognition of the ID. Additionally, due to the transition radiation, the TRT gives a complementary electron identification to the calorimeter.

\subsubsection{Calorimeters}

The calorimeters of ATLAS are located next to the ID and its surrounding solenoid. Their purpose is to measure the energy of particles. For this reason, the particles are stopped and the deposited energy is measured. The calorimeters have to be thick enough to absorb almost all high energy particles. The calorimeter system of ATLAS is separated into two parts, an electromagnetic and a hadronic calorimeter. The electromagnetic part is designed to measure the energy of electrons and photons. A good resolution for photons is especially important to e.g. distinguish between two photons originating from a $\pi^{0}$ or a Higgs decay. The hadronic calorimeter has a much higher stopping power, so that all jets should be contained in it. A schematic view is shown in figure 3.8.

\section{The Electromagnetic Calorimeter}

The Electromagnetic Calorimeter is the inner one of the two calorimeter systems of ATLAS and located next to the TRT, only with the solenoid in between. It is a sam- 


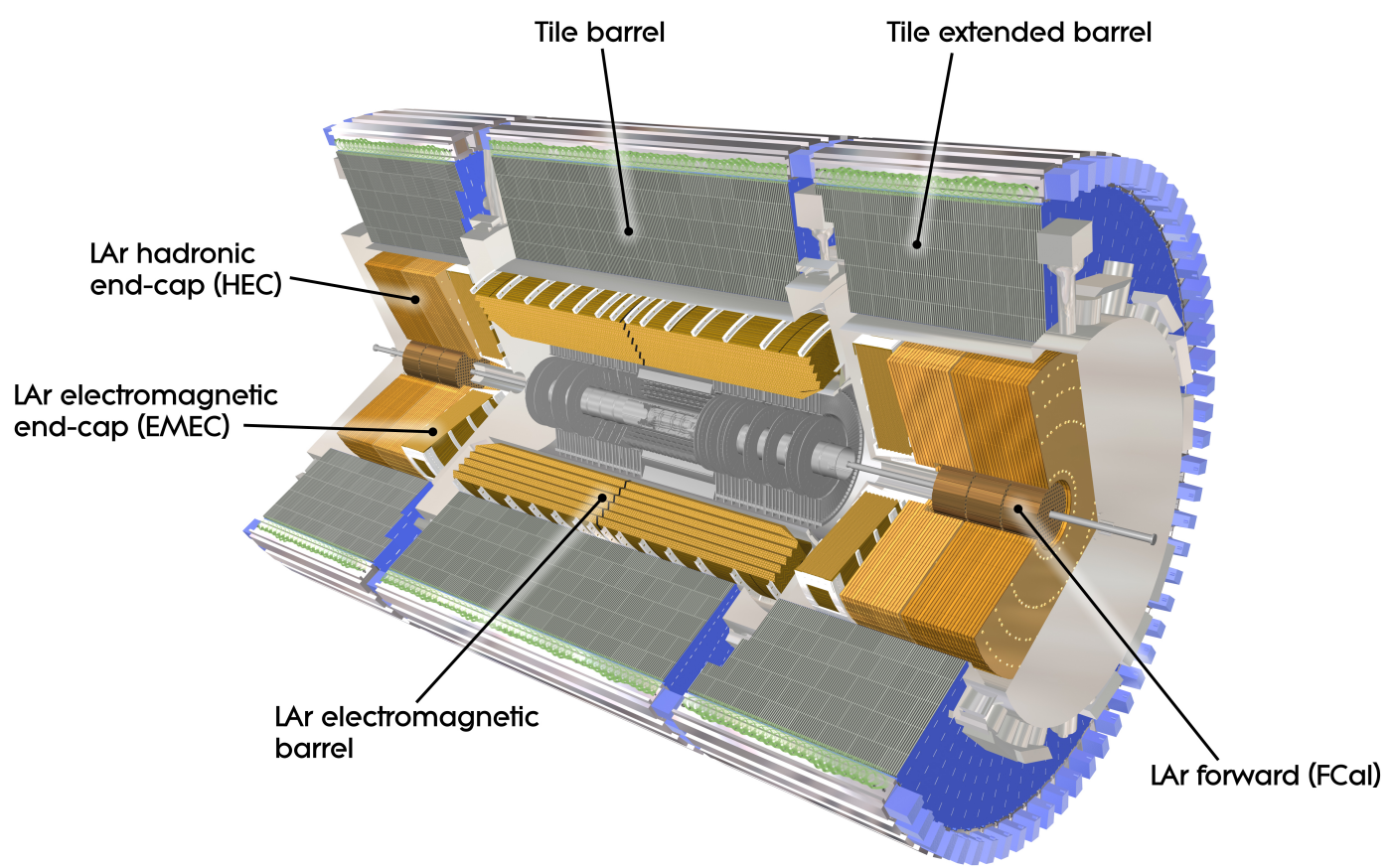

Figure 3.8.: Schematic view of the calorimeters of ATLAS. The Inner Detector is shown in grey in the centre [30].

pling calorimeter, which means that it consists of an absorbing and a detecting - sampling - material. The absorbing material is lead. As a material for signal generation, liquid argon (lAr) is used. The signal is generated by ionisation and it is read out with electrodes on kapton. The electrodes and the absorber material are accordion-shaped. This allows a segmentation in radial direction. The folding angle and spacing of the accordion structure is not constant for the whole detector volume, but changes with distance from the IP. Layers closer to the IP have a higher segmentation than outer layers, to increase the resolution.

Like other detector components, the electromagnetic calorimeter is separated into a barrel and an end cap part yielding a coverage of up to $|\eta|<3.2$. The end caps themselves are further divided into an inner and an outer wheel. They differ in the fact, that the inner wheel has a coarser granularity and only two instead of three active layers in depth.

\section{The Hadronic Calorimeter}

The Hadronic Calorimeter consists of three sub detectors, the Tile calorimeter, the Hadronic End-cap Calorimeter (HEC) and the Forward Calorimeter (FCal). From the 


\section{LHC \& ATLAS}

barrel region the Tile calorimeter extends up to $|\eta|<1.7$, which is the end of the full calorimeter extension. It is divided into a barrel and two extended barrel parts. The Tile calorimeter is a sampling calorimeter using steel plates as an absorber material and scintillator as the active part. In order to read out the scintillation light, wavelength shifting fibres are used, which direct the light to photomultipliers. The three parts of this calorimeter are divided into 64 parts azimuthally and into three parts radially.

The HEC is located directly behind the end cap part of the electromagnetic calorimeter and covers a range of $1.5<|\eta|<3.2$. It consists of four wheels, two for each side, which are segmented in depth into two parts each. The absorbing material are copper plates, interleaved with $\mathrm{Ar}$ as the active medium. The signal is read out via electrodes in the middle of the $8.5 \mathrm{~mm}$ gap between two copper plates.

The FCal is the most forward calorimeter in ATLAS covering an $\eta$ range of $3.1<|\eta|<$ 4.9. As it is only $4.7 \mathrm{~m}$ away from the IP, it is exposed to a very high particle flux. For this reason the active material of this calorimeter, which is lAr, fills only in a small gap of a coaxial cable. These cables are orientated parallel to the beam axis and organised in a metal matrix, which serves as the absorbing material. The absorbing material and the rod of the coaxial cable are either made of copper or of tungsten.

\subsubsection{The Muon System}

The Muon System of ATLAS is the outermost detector layer and also, in terms of physical size, the largest. It is contained in a toroidal magnetic field (see section 3.2.4). Behind the calorimeter the only detectable particles should be muons, as all other detectable particles should have been stopped in the calorimeters. Mouns are important for numerous physics processes and yield a good trigger. This is the reason why the muon system in general consists of a part which measures the trajectory of muons as accurately as possible and a part which generates a fast trigger signal. A schematic view of the muon system is shown in figure 3.9.

Monitored Drift Tubes (MDT) are used in order to track muons up to $|\eta|<2.7$. These are modules which consist of three to eight layers of drift tubes. The aluminium drift tubes have a diameter of nearly $30 \mathrm{~mm}$ and in the centre a $50 \mu \mathrm{m}$ thick tungsten-rhenium wire. They are filled with mostly argon gas and operated at $3080 \mathrm{~V}$. The MDT modules in the barrel region, which extends up to $|\eta|<1.4$, are mounted cylindrically in three layers around the beam pipe, in the end cap region in three disc layers perpendicular to the beam. The only region without MDT modules mounted is the first layer of the end cap discs in the region $2<|\eta|<2.7$. In this case Cathode Strip Chambers (CSC) are used instead. These multi wire proportional chambers are able to keep up with counting rates of up to $1000 \mathrm{~Hz} / \mathrm{cm}^{2}$ compared to the maximum rate of the MDTs of $150 \mathrm{~Hz} / \mathrm{cm}^{2}$.

The detectors for fast trigger generation extend up to $|\eta|<2.4$. Resistive Plate Chambers (RPC) are used for the barrel region until $|\eta|<1.05$. Thin Gap Chambers 


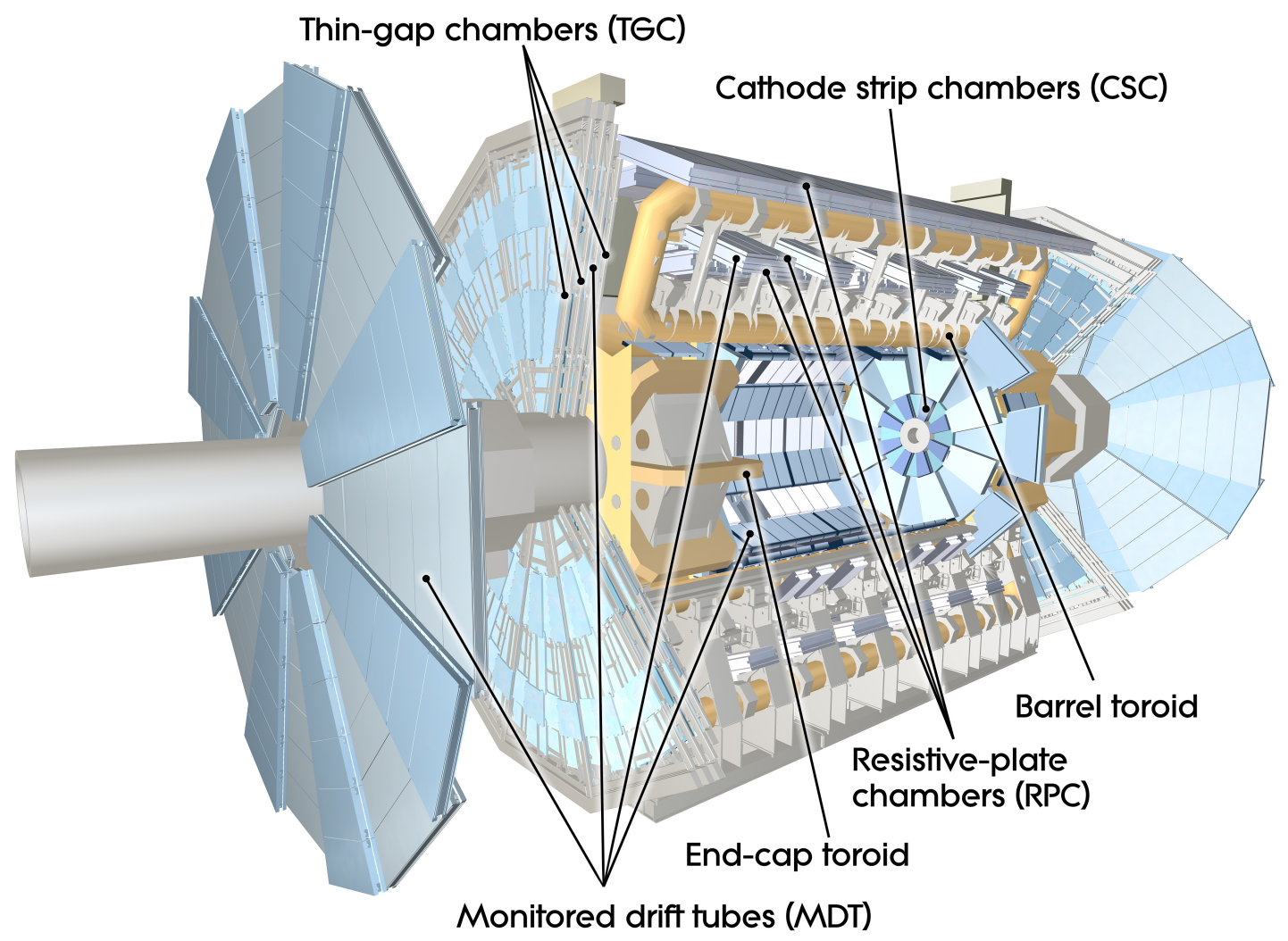

Figure 3.9.: Schematic view of the muon system of ATLAS [30].

(TGC) are located in the end cap region. Both are able to register the incoming muons within $25 \mathrm{~ns}$.

\subsubsection{The Magnet System}

The magnet system of ATLAS consists of an inner solenoid and an outer toroidal magnet. The central solenoid generates a $2 \mathrm{~T}$ magnetic field parallel to the beam pipe. It is located between the ID and the electromagnetic calorimeter. For this reason its material budget of approximately 0.66 radiation lengths has to be kept low. As it is a superconducting magnet made of $\mathrm{NbTi}$ it has to be cooled to $4.5 \mathrm{~K}$ for operation. A current of $7.73 \mathrm{kA}$ is needed to achieve the magnetic field strength of $2 \mathrm{~T}$.

The toroidal magnets enclose the muon system. They are separated into a central barrel part and end caps, which are inserted into the barrel part and line up with the solenoid magnet. Each of those three toroid magnets consists of eight air core coils, which provide a $0.5 \mathrm{~T}$ and $1 \mathrm{~T}$ magnetic field in the barrel and end cap region, respectively. The conducting part of the coils is made of $\mathrm{Nb} / \mathrm{Ti} / \mathrm{Cu}$ and will be supplied in total with 
$41 \mathrm{kA}$. As a consequence of the air core, ATLAS has a relatively low weight for its overall size.

\subsubsection{Data Acquisition and Trigger}

With an event size of approximately 1.3 Mbyte and the LHC running at nominal $40 \mathrm{MHz}$, the ATLAS detector would produce about 50 Tbyte per second. This is far too much to store or even transmit. For this reason not every, but only selected, interesting events are recorded. A trigger system is used in order to decide which event will be stored. This system consists of three levels, namely L1, L2, and the event filter. Each level reduces the event rate further. For example, the L1 trigger only uses information from the muon system and parts of the calorimeter, but no tracking information from the ID. It reduces the rate from $40 \mathrm{MHz}$ to a maximum of $75 \mathrm{kHz}$. Hit information from the ID is read out only if the event passes the L1 filter and then used for the L2 trigger. In the event filter step offline analysis is performed and the event rate is reduced to about $200 \mathrm{~Hz}$.

\subsubsection{Diamond detectors in ATLAS}

Besides the major detection systems described above, there are several small experiments inside of ATLAS and in the far forward region, which are, with exceptions, not used to measure a collision event, but merely to monitor the beam and the luminosity. Two of these monitors use diamond sensors. Installed since construction is the Beam Conditions Monitor (BCM) [38] and since the shut down prior to Run II the Diamond Beam Monitor (DBM) [39]. Both are located in the forward region close to the beam pipe inside the ID package.

The BCM consists of four diamond sensors on each side of the IP which are mounted circularly around the beam pipe. The distance to the beam pipe is only $5.5 \mathrm{~cm}$ so that the diamonds have to withstand radiation doses of $500 \mathrm{kGy}$ during ten years of operation. The diamonds themselves are pCVD diamonds and have a size of $1 \times 1 \mathrm{~cm}^{2}$. They have one large planar electrode, so that only the current of charges induced by the stream of particles can be measured, but not individual particles.

The DBM is located between the BCM and the IP, about one meter away from the collision region. It consists of four telescopes on each side with three sensors each. The sensors have an active region of $20.0 \times 16.8 \mathrm{~mm}^{2}$ segmented into 26,880 pixels. Out of these 24 sensors, 18 are pCVD diamonds and six are planar silicon sensors. Each telescope is equipped with either of these sensor types exclusively. The four telescopes on each side are mounted circularly close to the beam pipe. The three sensors in a telescope are mounted in a projective geometry, pointing at the interaction point. This allows for coincidence tracking of particles and the distinction if the tracks originate from the IP or are part of the beam halo. 


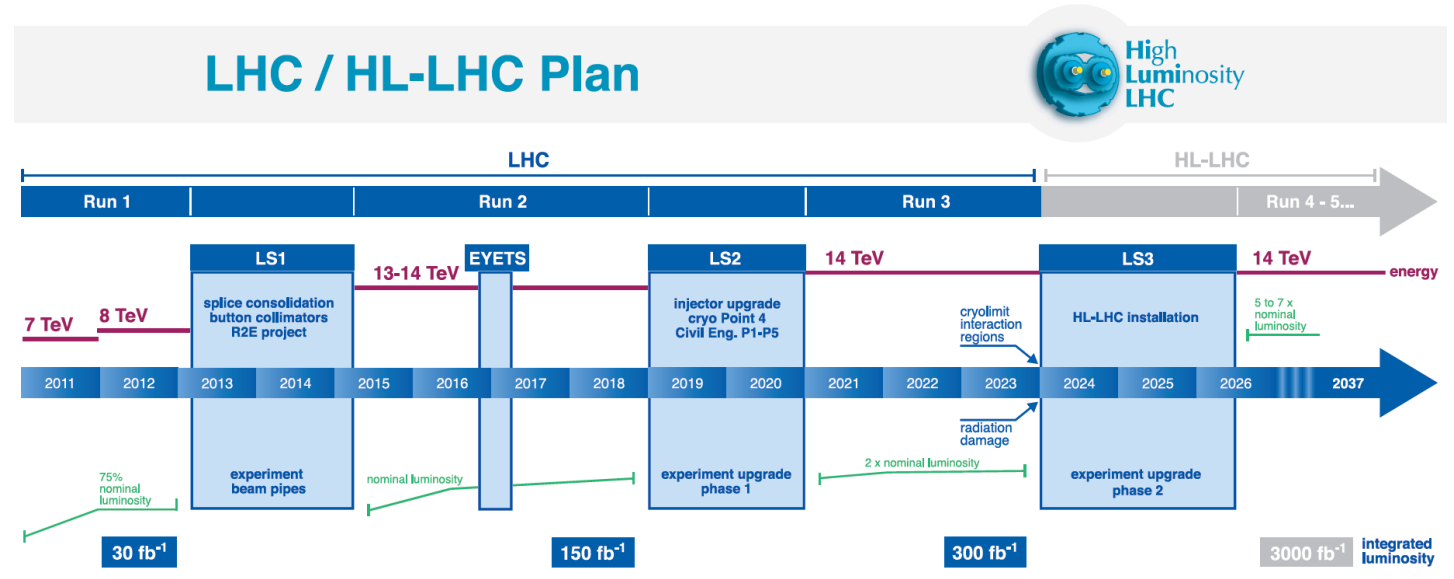

Figure 3.10.: Upgrade and operation schedule of the LHC [40].

\subsection{Upgrade Plans for LHC and ATLAS}

In order to measure even the smallest deviations of processes from the standard model prediction, more data at a higher rate has to be collected. This means increasing the luminosity of the LHC far beyond its design value. This project is called High Luminosity$L H C$ (HL-LHC). The current plans are to increase the luminosity by a factor of five to seven with respect to the design value at a centre of mass energy of $14 \mathrm{TeV}$. Neither the LHC nor all components of the experiments are able to handle such an increase in particles and radiation damage. This means that major parts of them have to be upgraded or replaced. The schedule for this upgrade and operation process is shown in figure 3.10 .

For ATLAS the increased luminosity means a full replacement of the ID [31]. By the time Run II ends, the existing sensors will be at the end of their lifetime. Also an increase in luminosity means more hard interactions per bunch crossing (pile-up). This number will rise from a mean value of 20 at the end of Run I up to 200. To distinguish particles from the different primary vertices, a higher resolution of the tracking is needed. The read out of the sensors has to be able to cope with a higher occupancy per sensor. These requirements, radiation damage and higher resolution, necessitate the full replacement of the ID.

The replacement for the ID is called Inner Tracker (ITk). It will only consist of pixelated and strip sensors, while having the total dimensions of the current ID. For the layout, several scenarios are discussed, with some scenarios reaching a track coverage of up to $|\eta|<4$. For such a detector four and five full barrel layers and twelve and seven end cap discs on each side of pixel and strip sensors are needed, respectively [31]. This yields an active area of $8.2 \mathrm{~m}^{2}$ for pixels and $193 \mathrm{~m}^{2}$ for strips. Combining the large 


\section{LHC \& ATLAS}

active area of the new pixel detector with a minimum fluence of $1.4 \times 10^{16} \mathrm{n}_{\mathrm{eq}} \mathrm{cm}^{-2}$ for the innermost layer after ten years of operation, several sensor technologies have to be used for the detector. For the outer barrel layers, different sensor concepts are considered with potentially lower costs. These are for example four chip planar modules or HV-CMOS sensors which are glued onto a read out chip. For the innermost layer a sensor technology which is very radiation hard has to be chosen. Due to the relatively small active area of this barrel layer, diamond is a sensor candidate for this as it has a high radiation tolerance.

Not only the ID will be fully replaced for the HL-LHC upgrade, but the whole detector will be improved. A new and faster trigger system will be put in place, which then also takes tracking for its first trigger decision into account. The forward calorimeter will be upgraded to have a finer granularity and the trigger systems of the muon spectrometer will also have a higher segmentation and coverage. 


\section{CHAPTER 4}

\section{Silicon Pixel Detectors}

Silicon is the de facto standard sensor material for pixel sensors in high energy physics. Every other potential sensor material has to improve from this in at least one key aspect, like e.g. signal to noise ratio or detection probability of photons. In case of diamond sensors the major advantage is the higher radiation tolerance. Diamond sensors also have the advantage, that they can easily use most of the read out electronics which were originally developed for silicon sensors. Therefore this chapter introduces the concept of pixel detectors with silicon as a sensor material, before in Chapter 5 the differences and advantages of diamond sensors are explained.

First, the energy loss of particles in matter is described in section 4.1, which applies to both diamond and silicon sensors. Then in section 4.2 the signal generation in solid state sensors is explained and the read out of it for silicon sensors. In section 4.3 the Pixel Detector of ATLAS is described as an example of a pixel detector system.

\subsection{Energy Loss of Particles in Matter}

How particles lose energy in matter depends on the type of particle and its energy. For tracking detectors at collider experiments mainly the energy loss of charged particles is relevant. This is described in section 4.1.1 for heavy particles and in section 4.1.2 for electrons. While uncharged particles, such as neutrons, do also lose energy in matter through other processes e.g. scattering at nuclei, which cross sections are small compared to those of charged particles. Consequently, this is typically neglected for pure tracking detectors. The only neutral particle which has to be considered is the photon. Its interactions with matter are described in section 4.1.3. 


\section{Silicon Pixel Detectors}

\subsubsection{Energy Loss of heavy charged Particles in Matter}

In order to calculate the energy loss of charged particles in matter, they have to be separated into two categories. One group consists solely of the electron ${ }^{1}$, while the other bundles all other heavier charged particles up to light nuclei. In general, all charged particles follow the same physical laws, but as the electron is light compared to all other charged particles, other effects than for heavier particles dominate. These are described in section 4.1.2.

Heavy charged particles mainly interact with matter through inelastic collisions with shell electrons or through elastic scattering with nuclei [41]. As the masses of the nuclei are large with respect to masses of the particles, the transferred energy in elastic collisions is very small. Accordingly, almost all of the energy loss can be attributed to the inelastic collisions with shell electrons. Other processes like Cherenkov radiation, nuclear reactions and bremsstrahlung may occur, but are very rare ${ }^{2}$. Out of the two main processes, the inelastic collisions contribute the largest share to the energy loss. In these collisions, the electrons are either excited or, if the transferred energy is large enough, the atom is ionised. The generated free electrons can even have enough energy to excite other shell electrons and are refered to as $\delta$-electrons.

The transmitted energy from one single collision with a shell electron or a nuclei cannot be precisely determined, as it is a statistical process. However, as the density of atoms in solid state matter is very high, many of these processes occur, so that a mean amount of energy loss per distance can be calculated. The formula which describes the energy loss through inelastic collisions is known as the Bethe-Bloch formula which is given below.

$$
-\left\langle\frac{d E}{d x}\right\rangle=2 \pi N_{a} r_{e}^{2} m_{e} c^{2} \rho \frac{Z}{A} \frac{z^{2}}{\beta^{2}}\left[\ln \left(\frac{2 m_{e} \gamma^{2} v^{2} W_{\max }}{I^{2}}\right)-2 \beta^{2}-\delta-2 \frac{C}{Z}\right]
$$

The parameters of this formula are explained in table 4.1. As the energy loss is a statistical process, $\langle d E / d x\rangle$ gives the mean value. This value is not identical with the most probable value, as the energy loss follows a Landau distribution. Therefore the most probable value is lower than the mean value with the median value in between.

Figure 4.1 shows the mean energy loss for anti-muons in copper over a wide momentum range. For momenta of $\beta \gamma<0.1$ the Bethe-Bloch formula does not describe the energy loss any more. In this range the particles are very slow and might even be absorbed by the material. For values of $\beta \gamma>1000$ the formula also does not any more describe the dominant process of energy loss. In this case radiative losses like bremsstrahlung are dominating. Within the momentum range the Bethe-Bloch formula is valid, for low

\footnotetext{
${ }^{1}$ As the positron has the same mass, it behaves in this respect like the electron. Throughout this chapter it is also implicitly meant, if speaking of electrons.

${ }^{2}$ Cherenkov radiation is used by specialised detectors, but merely for particle identification rather than for tracking.
} 


\begin{tabular}{ll}
\hline Symbol & Explanation \\
\hline$N_{a}$ & Avogadro's number \\
$r_{e}$ & classical electron radius \\
$m_{e}$ & electron mass \\
$c$ & speed of light \\
$\rho$ & density of absorbing material \\
$Z$ & atomic number of absorbing material \\
$A$ & atomic weight of absorbing material \\
$z$ & charge of incident particle in units of $e$ \\
$\beta$ & $v / c$ of the incident particle \\
$v$ & velocity of the incident particle \\
$\gamma$ & $1 / \sqrt{1-\beta^{2}}$ \\
$W_{\max }$ & maximum energy transfer in a single collision \\
$I$ & mean excitation potential \\
$\delta$ & density correction \\
$C$ & shell correction \\
\hline
\end{tabular}

Table 4.1.: Explanation of the parameters of the Bethe-Bloch formula given in Equation $4.1[41]$.

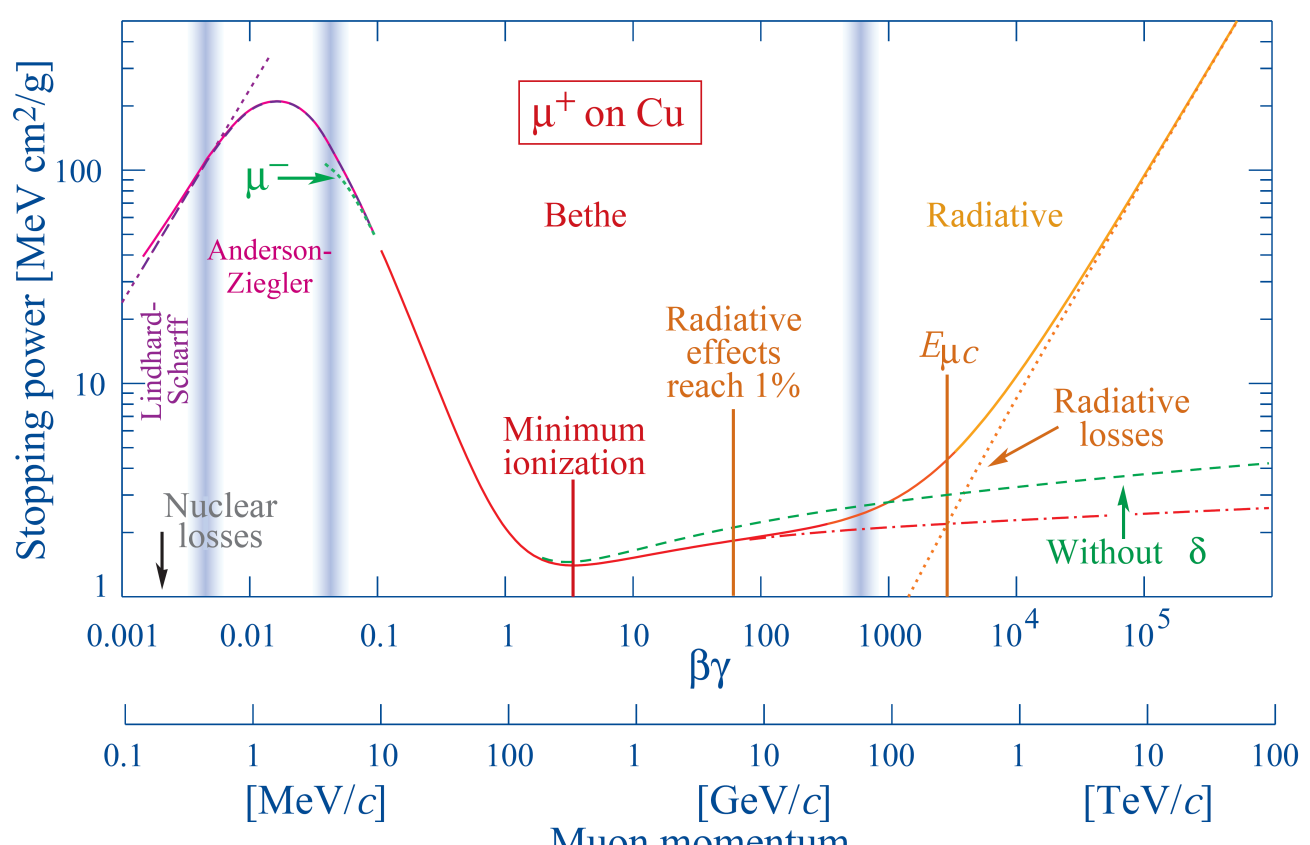

Figure 4.1.: Energy loss of anti-muons in copper over a wide momenta range [10]. 


\section{Silicon Pixel Detectors}

momenta the energy loss decreases with $1 / \beta^{2}$ until a minimum at $\beta \gamma \approx 3-4$ is reached. Particles with such momenta deposit a minimal amount of energy in the detector and are therefore called minimum ionising particles (mip). The energy loss does only slightly change for higher momenta, as it only increases logarithmically. For this reason the en-

ergy loss of a mip of $-\left\langle\frac{d E}{d x}\right\rangle_{\text {mip }} \approx 1.5 \mathrm{MeV}^{\mathrm{cm}} \frac{\mathrm{cm}^{2}}{\mathrm{~g}}$ is a good approximation also for particles with a higher momentum.

The particles also collide elastically with the nucleii and are as a result slightly deflected. For tracking purposes this is an unwanted effect, as it reduces the resolution of a detector. Therefore the sensors of tracking detectors should be as thin as possible, while still obtaining enough signal. The deflection originating from scattering at a single nuclei is described by Rutherford's formula [41]. For a large number of independent scatterings the distribution of the final scattering angle can be approximated with a Gaussian distribution. The mean of the distribution is at zero degrees and the standard deviation, if projected on a plane, is empirically given by [10]

$$
\sigma_{\theta}=\frac{13.6 \mathrm{MeV}}{\beta c p} z \sqrt{x / X_{0}}\left[1+0.038 \ln \left(x / X_{0}\right)\right]
$$

with $x$ being the thickness of the material and $X_{0}$ its radiation length, which is explained in Section 4.1.2.

\subsubsection{Energy Loss of Electrons in Matter}

Electrons have a very low mass compared to other charged particles. This results in much higher momenta for electrons of the same energy. These momenta are that high, that the Bethe-Bloch formula does not describe the major process of energy loss any more. Instead, electrons with an energy of a few 10's of MeV lose their energy in matter predominantly through radiative losses. Due to deflection in the electric field of nuclei, the electron emits a photon. This process is called bremsstrahlung.

In order to quantify the energy loss of electrons through bremsstrahlung in different materials, the quantity radiation length $X_{0}$ is used. For a particle with an initial energy of $E_{0}$, the remaining energy $E$ after traversing a material with a thickness of $x$ can be calculated via [41]

$$
E=E_{0} e^{-x / X_{0}}
$$

Accordingly, $X_{0}$ is the distance in a material after which an incoming electron has only $1 / e$ of its initial energy left. The radiation length is material dependent and used to quantify the amount of material of a detector. Since more material means more scattering of the particles, tracking detectors, including the support structures, should have a low $X_{0}$, while calorimeters should have a high enough $X_{0}$ to ensure that all particles can be stopped inside the calorimeter. 


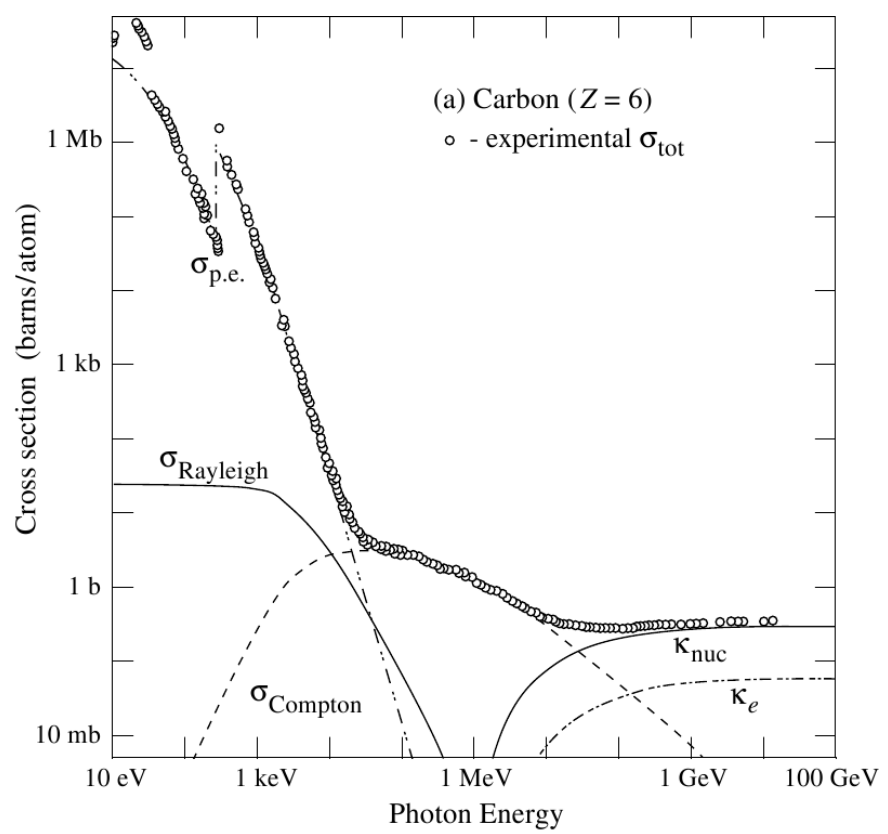

Figure 4.2.: Photon cross section in carbon [10]. $\sigma_{\text {e.p. }}$ is the cross section for the photoelectric effect, $\sigma_{\text {Compton }}$ for Compton scattering, and $\kappa_{\text {nuc }}$ and $\kappa_{e}$ for pair production in the field of the nuclei or electrons, respectively. $\sigma_{\text {Rayleigh }}$ is the cross section for Rayleigh scattering, where the energy of the photon is not altered.

\subsubsection{Energy Loss of Photons in Matter}

Photons interact with matter in mainly three ways, which are, ordered by the minimum photon energy needed:

1. Photoelectric effect

2. Compton scattering

3. Pair production.

The cross sections with respect to the energy are shown in figure 4.2.

One distinct feature of these interactions is that two of these do not attenuate the energy of the photon but absorb it entirely. Only Compton scattering changes the energy and the direction of the photon. Consequently, for a photon beam not the change in energy, but for intensity with respect to the distance in matter is quoted. It can be calculated via the formula

$$
I(x)=I_{0} e^{-\mu x}
$$




\section{Silicon Pixel Detectors}

with $I(x)$ being the intensity after a distance of $x$ through a material with absorption coefficient $\mu$ for a beam with an initial intensity of $I_{0}$.

Photons originating from collisions at high energy colliders are high energetic. Consequently, the most relevant process is pair production. In this case the photon decays in the electric field of a nucleus into an electron-positron pair. For this reason the minimum energy of the photon for this process is two times the mass of an electron. At photon energies in the order of 10 's of $\mathrm{MeV}$ pair production becomes the dominating process.

In a tracking detector a signal from photons is typically unwanted, because, as stated above, in most cases the photon is absorbed in this process. This renders tracking of photons impossible as it can be only measured at one point. This is typically not considered as a problem for a detector at a high energy particle accelerator, as the energy of the photon is the interesting quantity. Accordingly, the tracking system and its support structures should be as transparent as possible for photons, meaning a low total $\mu$, so that the photons can reach the electromagnetic calorimeter.

\subsection{Signal Generation and Detection in Silicon}

This section focuses on signal generation in the detector and how it can be read out, especially in the case of silicon. For this reason in section 4.2 .1 basic solid state physics for the relevant sensor materials, i.e. silicon and diamond, is explained. In section 4.2.2 the read out of the deposited signal is discussed.

\subsubsection{Semiconductor Basics}

Diamond and silicon have the same crystal structure ${ }^{3}$. Both atoms, carbon in case of diamond, have four electrons in the outermost shell. The preferred electron configuration for the outermost shell is eight electrons. With each electron on the outermost shell it is possible to form a covalent electron bond with an outer electron of a neighbouring atom, so that the two electrons are shared between the atoms. Forming such a bonding with all four outer electrons, the atoms are able to achieve the ideal configuration. For this reason, carbon and silicon can build stable crystals with atoms of the same kind.

The allowed energy levels for the outer electrons are equal for atoms of the same kind. But if the atoms are very close, like in a crystal structure, the energy levels are slightly shifted due to the potential shift caused by neighbouring atoms. The shift is so small, that single energy levels cannot be distinguished any more. Instead they form energy bands of allowed states. The origin of this can be understood by assuming the spacing of the crystal being first relatively large. In this case every atom has the exact same energy levels, as the atoms do not interfere with each other. Bringing the atoms closer together, they cannot be treated as independent atoms any more, but as a single

\footnotetext{
${ }^{3}$ This is also true for germanium.
} 
quantum mechanical system. With the same energy levels for every atom, they violate Pauli's principle. According to this, two electrons cannot be in the exact same state in a system. But as the energy levels degenerate due the influence of the neighbouring atoms on the potential, Pauli's principle is not violated [42].

In the ground state, i.e. at $T=0 \mathrm{~K}$, the highest filled energy band is called valence band, the lowest unfilled band conduction band. Electrons in the valence band are tightly bound to their atoms and cannot move freely in the crystal. Electrons in the conduction band are able to do this. Depending on the crystal structure, there might be an energy gap between these two bands, where no states for electrons exist. In general, crystals can be categorised depending on the size of this energy gap. If the valence and conduction band overlap, i.e. there is no energy gap, the electrons can move from one band to the other without extra energy. Such a material is called a conductor. Materials with only a partially filled valence band belong also in this category. If the energy gap is greater than $4 \mathrm{eV}$, the electrons need more energy than they would normally get through thermal excitation to cross the gap [43]. As a result these materials are not conductive and are called insulators. For materials with a small energy gap of $4 \mathrm{eV}$ or less, thermal excitation of an electron in the conduction band is possible, yielding a conductivity between conductors and insulators. Such materials are called semiconductors.

Silicon has a band gap of $1.12 \mathrm{eV}$ and is therefore a semiconductor. But the mean energy needed to excite an electron from the valence to the conduction band is $3.6 \mathrm{eV}$. The reason for this is that silicon is an indirect semiconductor. Indirect means, that the highest energy states of the valence band and the lowest energy states of the conduction band do not have the same momenta. Therefore, the electrons either need more energy to change bands without changing their momenta or they do need additional energy to actually alter their momenta.

When the electrons change from the valence to the conduction band, they can move like free charge carriers. In the valence band they leave an unfilled state behind. This state is known as a hole. These holes can also move in the crystal, as electrons from neighbouring atoms fill the hole and therefore change its position. Holes act as positive charge carriers, whereas electrons act as negative charge carriers.

\subsubsection{Operating Silicon as a Solid State Detector}

In a pure silicon crystal at a temperature of $300 \mathrm{~K}$ there are about $1.5 \times 10^{10} \mathrm{~cm}^{-3}$ electrons in the conduction band and as many holes in the valence band [41]. Considering a mip particle hitting only one pixel with the size of $250 \times 50 \mu \mathrm{m}^{2}$ and a sensor thickness of $250 \mu \mathrm{m}$ it creates approximately 24.300 electron hole pairs. Comparing that to approximately 46, 900 electron hole pairs from thermal excitation in the same volume, such a signal is not easy to detect, even though the electron hole pairs from thermal excitation can be reduced by cooling the sensor. Nevertheless, this calculation assumes that every pixel has the exact same potential. Due to the fabrication process of the electrodes, 


\section{Silicon Pixel Detectors}

this assumption is not valid. Instead, the slightly different potential for individual pixels leads to an increase of current for some pixels and as a result to an increase of noise.

For this reason a detector consisting of a pure silicon crystal is difficult to operate. A solution is to dope the silicon and create a pn-junction. Doping a semiconductor means adding atoms which have one more or one less electron in the outer shell into the material. This can either be done during the growth of the semiconductor or later using ion beams. Atoms with one electron more in the outer shell, i.e. in total five electrons, are named donor, the resulting material n-type semiconductor. The energy level of the extra electron is very close to the conduction band. Therefore, this electron is easily excited into the conduction band. Semiconductors doped with with atoms which have one electron less in the outer shell are named $p$-type semiconductors. The doped atom acts as an acceptor, as it has a energy level just over the valence band. For this reason, an electron is easily excited into this level and leaves a hole behind.

Putting an n-type and a p-type semiconductor together creates a pn-junction. In the n-type semiconductor the concentration of electrons is higher. The same is true for holes in a p-type semiconductor. Consequently, at the interface between these two semiconductors there is a concentration gradient of electrons and holes. This is compensated by diffusion of electrons to the p-type material and of holes to the n-type material. The diffusion leads to a negative space charge in the p-type semiconductor. Accordingly, the n-type becomes positively charged. Due to this charge, a potential builds up, which eventually stops the diffusion of charges. This region is named depletion region and is free of mobile charge carriers. If electron hole pairs are created by ionising radiation, these carriers are dragged out of the region by the electric field. Placing electrodes at the edges of the depletion region, makes it possible to measure the resulting current. The size of such an intrinsic depletion region is typically not as large as a sensor is thick. In order to enlarge this region, a reverse bias voltage can be applied. The reverse bias voltage reduces the potential originating from the space charges and thus the electric field. This allows charge carriers to diffuse further into the opposing material and thus enlarges the depleted region. Assuming, that one doping concentration is much higher than the other, the depleted region predominantly extends into the lesser doped material. Its thickness $x$ can be approximated as [44]

$$
x \approx \sqrt{\frac{2 \epsilon_{0} U}{e N}}
$$

with $\epsilon_{0}$ being the dielectric constant, $U$ the applied voltage and $N$ the doping concentration of the lesser doped material.

The electron hole pairs, created by traversing radiation, drift towards the electrodes, due to the electric field. According to the Shockley-Ramo theorem, the charges induce a current on the electrode while drifting towards it $[45,46]$. The induced current can be calculated via

$$
i=q \vec{v} \vec{E}_{W}
$$


where $i$ is the instantaneous induced current, $q$ the total charge of the charge cloud, $\vec{v}$ its velocity and $\vec{E}_{W}$ the weighting field. The weighting field depends only on the geometry of the electrodes and due to its normalisation, the integral of the induced current over the drift time equals the total charge of the charge cloud [44].

As the recorded signal is typically small, i.e. in the order of a few thousand electrons, it needs to be amplified. For this reason the read out electrode is connected to an amplifier. The amplifier converts the charge signal into a voltage signal, which is much easier to propagate in modern electronics. In order to prevent that the amplifier amplifies the charge of multiple particles, it has to be reset. This is either done via a resistance or a current source. While the current source results in a linear decrease of the voltage signal, the resistor results in an exponential decrease.

In order to suppress signals originating from noise, the voltage signal usually has to pass a discriminator. This is done in order to reduce the number of signals, as in a typical pixel detector the number of channels is too large to read out every single channel for an event. The discriminator can also be used to further compress the information from the full signal shape by converting it to the time the signal stays over the threshold. This time over threshold (ToT) is proportional to the amplitude of the signal, if the decrease of the voltage signal is linear.

\subsection{The ATLAS Pixel Detector}

The ATLAS Pixel Detector is the innermost subdetector of ATLAS [47]. It is separated into three barrel layers and three end cap layers on each end of the barrel. During the LHC shutdown in 2014, an additional innermost fourth barrel layer, the Insertable $B$-Layer (IBL), was installed [48]. A drawing of the Pixel Detector without the IBL is shown in figure 4.3.

The Pixel Detector is a tracking detector, used to resolve the primary vertices of the tracks. At the end of Run I, up to 40 primary interactions per event had to be resolved. A good resolution of the vertices is particularly important for the identification of bquarks in a decay. These b-quarks live long enough to move away from their primary vertex before decaying. Its decay products originate from a secondary vertex, which is in the order of several hundred micrometres apart from the primary vertex [49]. The identification of b-quarks, called b-tagging, is particularly important for all analysis involving a top-quark. These decay almost exclusively into a b-quark and a W-boson. A good b-tagging reduces the complexity of the reconstruction of such an event and reduces the background.

In order to achieve a high resolution, the Pixel Detector consists of more than 80 million single pixels covering an active area of approximately $1.7 \mathrm{~m}^{2}$. They cover a pseudo rapidity range of up to $|\eta|<2.5$. The IBL further adds about six million pixels 


\section{Silicon Pixel Detectors}

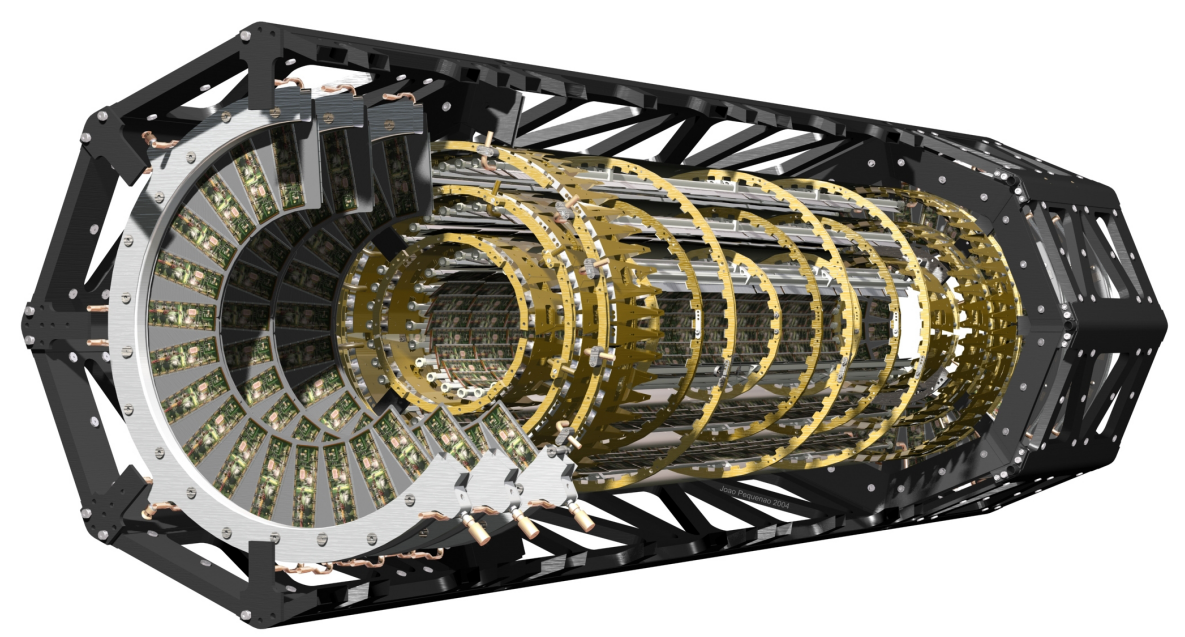

Figure 4.3.: Schematic view of the ATLAS Pixel Detector during Run I. The IBL, which was inserted for Run II, is not shown [47].

and $0.15 \mathrm{~m}^{2}$ of active area up to a pseudo rapidity of $|\eta|<3$.

\subsubsection{Sensor}

The ATLAS Pixel Detector uses n-in-n diffusion oxygenated silicon sensors. This means that the silicon bulk is slightly n-doped and read out via a heavily n-doped electrode on one sensor surface. In order to create a pn-junction and to deplete the sensor, a depletion voltage is applied via a p-doped electrode on the other surface. Accordingly, the depletion region grows from the back- to the frontside. This means that the sensor has to be always fully depleted for operation.

During data taking at the LHC, the sensors will suffer from radiation damage. This results for silicon in an enrichment of p-type defects up to the point, where the whole sensor bulk changes its effective doping from n-type to "p"-type. Such a process is called type inversion. Further irradiation increases the effective p-doping and consequently the potential needed to fully deplete the sensor. The dependency of the absolute depletion voltage on the radiation dose is shown in figure 4.4. This voltage can increase beyond the limits of the power supplies, which results in not fully depleted sensors. Unlike unirradiated n-in-n type sensors, operation of partially depleted type inverted sensors is still possible, as the depletion region grows from the read out n-type electrodes inside the "p"-type sensor.

As long as the sensor bulk is not type inverted, a good isolation between the n-doped pixels is crucial. For this reason, the region between the pixel implants, is slightly pdoped $[47,51]$. This doped area ensures, that the resistance between two pixel implants 


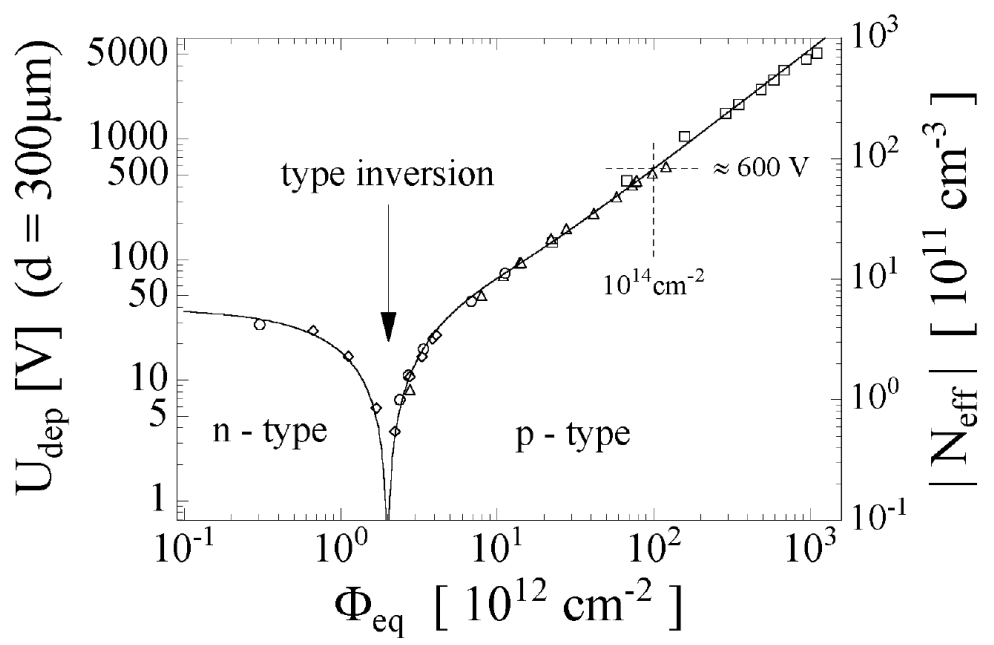

Figure 4.4.: Depletion voltage of ATLAS Pixel silicon sensors with respect to the accumulated fluence. With irradiation the effective doping concentration and type of the sensor bulk changes [50].

is high, so that they are not shortend. The edges of a planar silicon sensor need also special treatment. The drop of the potential on the p-doped side has to be moderated or otherwise discharges at the cutting edge of the sensor can occur [42]. Accordingly, 16 guard rings are implemented on the p-electrode side. These guard rings have a width of $10 \mu \mathrm{m}$ and consist of a p-implant and an aluminium contact. They surround the whole sensor area and have a distance between each other of $8 \mu \mathrm{m}$ to $15 \mu \mathrm{m}$ [52]. The rings are not interconnected and ensure a controlled drop of the potential towards the edge of the sensor. Due to the width of this structure and a safety margin for cutting the sensor, an inactive area of $1100 \mu \mathrm{m}$ width surrounds the sensor [53].

The sensor has an active area of $60.8 \times 16.4 \mathrm{~mm}^{2}$ and a thickness of about $250 \mu \mathrm{m}$. The n-type electrode is separated into 47,232 pixels, organised in 144 columns and 328 rows. Most of these have a size of $400 \times 50 \mu \mathrm{m}^{2}$. The only exceptions are pixels in 16 columns, which have a size of $600 \times 50 \mu \mathrm{m}^{2}$. The reason for this is, that the sensor is not read out with a single but with 16 front end chips. At the interface between two of these, the pixels have to be slightly larger. The maximum possible depletion voltage for the sensor is $600 \mathrm{~V}$. During operation, the sensor is cooled down to $-10^{\circ} \mathrm{C}$ in order to reduce the leakage current.

\subsubsection{Read out}

Each of the 1,744 silicon sensors is read out by 16 FE-I3 front end chips, which are grouped in two rows with eight FE-I3 each. Every FE-I3 is able to read out 2880 pixels individually, which are organised in 16 columns and 80 rows. 


\section{Silicon Pixel Detectors}

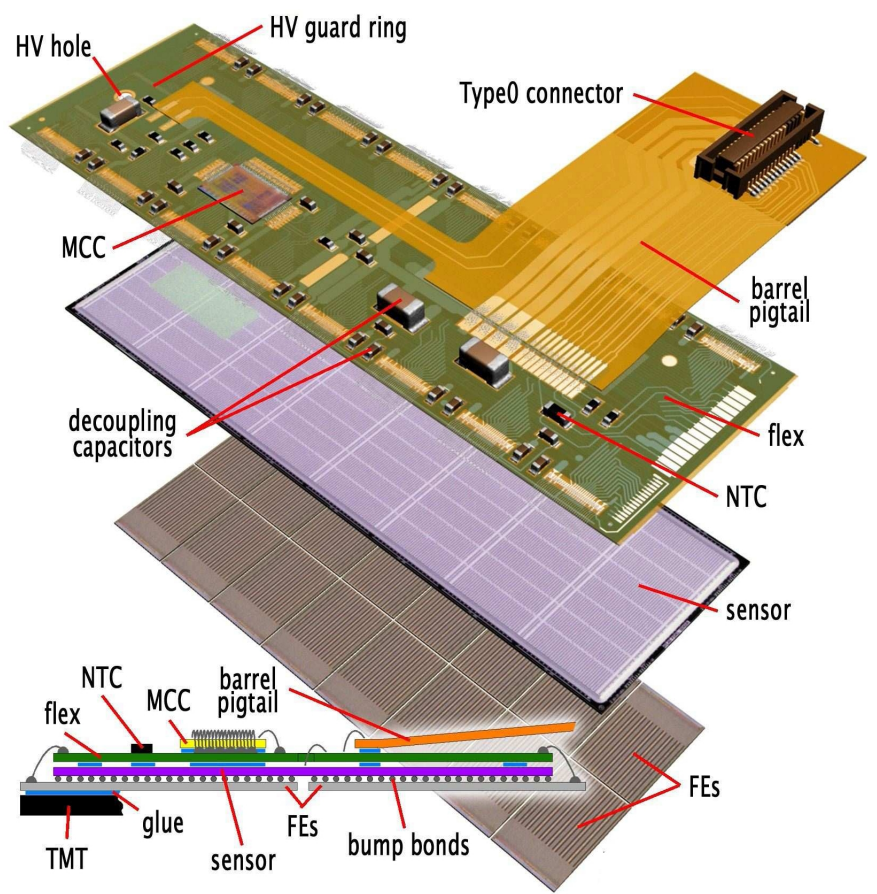

Figure 4.5.: Schematic view of a Pixel Detector module [47].

A full pixel module is completed with the Module Control Chip (MCC). It manages the communication with the front ends of a module, distributes trigger signals and processes the collected data. A schematic view of a complete pixel module is shown in figure 4.5.

\subsubsection{The Insertable B-Layer}

Originally, it was planned to replace the innermost layer of the Pixel Detector every three years of operation, as the performance decreases due to radiation damage [48]. During the construction of the Pixel Detector, this changed so that no layer can be replaced. Instead, during the shut down in 2014, an additional innermost pixel layer was installed, the Insertable B-Layer (IBL). It uses a new read out chip, the FE-I4, which has a smaller pixel size of $250 \times 50 \mu \mathrm{m}^{2}$ and is able to cope with the expected high occupancy. One FEI4 can read out 26,880 individual pixels, which are organised in 80 columns and 336 rows.

The IBL consists of 14 staves, which are mounted around the new, smaller beam pipe. Each stave contains 32 FE-I4. These are grouped into modules, which either consist of one or two front ends, depending on the sensor. Two different types of silicon sensors are used. The inner 24 front ends are connected to planar n-in-n sensors, with two front ends forming a module. At both ends of the staves, four $3 \mathrm{D}$ sensors are connected to one front end each. 
The planar n-in-n sensors are refined versions of the sensors used for the existing pixel detector [54]. These are $200 \mu \mathrm{m}$ thick, which yields a higher electric field for the same bias voltage. This is possible due to the higher resistivity of the bulk material, which is now commercially available. The width of the inactive area around the sensor is also reduced to $350 \mu \mathrm{m}$. It is achieved by reducing the number of guard rings to 13 and having an overlap of $250 \mu \mathrm{m}$ of the inner rings with the pixel implementation on the other side of the sensor. This slight overlap does not reduce the efficiency.

The 3D sensors use electrodes inside the bulk material, which is a new technique. For details on the 3D geometry, see chapter 5.4. The production of these sensors is very demanding. As a result, the size of one sensor corresponds to only one front end chip. 



\section{CHAPTER 5}

\section{Diamond Pixel Detectors}

Diamond has some unique properties, which make it an interesting sensor material. Especially its high radiation tolerance gives it an advantage over silicon sensors in specific regions of a detector. In these regions, the sensors are exposed to a high particle flux and suffer from heavy radiation damage. The innermost layer of a tracking detector at HL-LHC, which would be at a radius of few centimetres away from the beam, is such an environment.

The high radiation tolerance comes at a price, namely the lower deposited signal compared to silicon sensors. This can be compensated by the lower intrinsic noise and special electrode geometries. Such electrodes would be not on the surface of the diamond but also inside its bulk.

This chapter gives an overview of diamond as a sensor material and what is needed to achieve a working sensor for a pixel detector. Accordingly, section 5.1 gives a summary of the relevant properties of diamond and how they compare to silicon. Natural diamond is too expensive to use and so artificial diamond is the material of choice, its production presented in section 5.2. As the major advantage of diamond over silicon is its higher radiation tolerance, this is discussed in more detail in section 5.3. An overview over the different electrode geometries is given in section 5.4 and the production of electrodes inside the diamond bulk is described in section 5.5. One crucial aspect is the contacting of such electrodes, which is discussed in section 5.6.

\subsection{Diamond Properties}

Diamond consists solely of carbon atoms. These atoms have four electrons in their outermost shell. Their orbital hybridisation of the electrons is $s p^{3}$ [55]. The orbitals are 


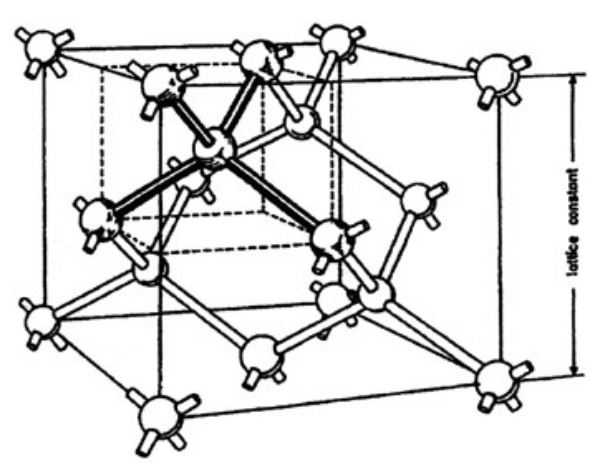

(a)

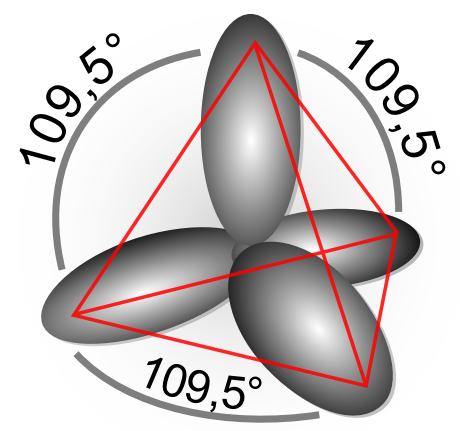

(b)

Figure 5.1.: (a) Schematic view of one cell of a diamond lattice [56] and (b) of a single carbon atom with its three $s p^{3}$ orbitals [57].

\begin{tabular}{l|ll}
\hline Property & Diamond & Silicon \\
\hline Band gap $(\mathrm{eV})$ & 5.5 & 1.12 \\
Intrinsic carrier density $\left(\mathrm{cm}^{-3}\right)$ & $<10^{3}$ & $1.5 \times 10^{10}$ \\
Dielectric constant & 5.7 & 11.9 \\
Displacement energy $(\mathrm{eV} /$ atom $)$ & 43 & $13-20$ \\
Energy to create e-h pair $(\mathrm{eV})$ & 13 & 3.6 \\
Radiation length $(\mathrm{cm})$ & 12.2 & 9.4 \\
Average signal created / $\mu \mathrm{m}(\mathrm{e})$ & 36 & 89 \\
\hline
\end{tabular}

Table 5.1.: Properties of diamond and silicon [58].

aligned in a tetrahedral way, so that the maximum angle between the orbitals is realised. Each of these orbitals overlaps with an orbital of the same kind from a neighbouring atom and consequently forms a stable bond. The crystal structure is a combination of two cubic face-centred crystals, shifted by a quarter of the cube diagonal (see figure 5.1(a)). This yields eight carbon atoms per unit cell. The enormous strength of the diamond cannot be explained by its crystal structure alone, as e.g. silicon has the same. The difference is the very high bond enthalpy of the carbon-carbon bond of $348 \mathrm{~kJ} / \mathrm{mol}$. This translates to a higher energy to remove an atom from the crystal lattice for diamond compared to silicon. As shown in table 5.1, this displacement energy is at least twice as high in diamond as in silicon [58]. As a result, this contributes to the radiation hardness of diamonds.

The radiation length of diamond is with $12.2 \mathrm{~cm}$ longer than the radiation length of silicon, which is $9.4 \mathrm{~cm}$. This is beneficial, as for a tracking detector the material bud- 


\subsection{Diamond Properties}

get should be as low as possible. However, since thinning diamonds below $500 \mu \mathrm{m}$ is technically challenging, diamond detectors tend to have a larger thickness than silicon sensors [59]. This compensates the advantage.

With a band gap of $5.5 \mathrm{eV}$ diamond is an insulator [58]. Like silicon, its band gap is indirect, which results in the required energy to create an electron hole pair of $13 \mathrm{eV}$. This is approximately 3.6 times more energy than for silicon. Combined with the longer radiation length, the average created signal per micrometer is $36 \mathrm{e}$ to $89 \mathrm{e}$ for diamond and silicon, respectively.

In order to be able to detect a signal, its amplitude should be sufficiently large compared to the noise, the so called signal to noise ratio. The lower signal from diamond sensors with respect to silicon sensors, does not conclusively result in a lower signal to noise ratio. The band gap of diamond is large enough to suppress thermal noise quite effectively. Additionally, the dielectric constant is only half as large as for silicon, which for the same electrode geometry results in half the detector capacitance with respect to silicon. This detector capacitance is one source for noise. For lower capacities, the noise is smaller. The very low number of intrinsic charge carriers in diamond leads to a behaviour similar to that of a depleted silicon sensor, with respect to this property. Accordingly, diamond does not need to be doped or depleted with a bias voltage. In practice, however, a high electric field is needed for a fast charge collection in diamond, as otherwise the charges would be trapped. Therefore, the applied bias voltage especially for low quality diamond sensors tend to be higher than for silicon sensors.

A major difference of diamond to silicon is the trapping of charges. In a perfect crystal, no states exist between the valence and the conduction band. However, in a real crystal, defects create localised states in the band gap. These states can trap a charge and prevent it from drifting further to the electrode. The average time before the charge carrier is detrapped depends on the energy of the state. If the charge is trapped longer than the time needed to collect the other charges, it is effectively removed from the signal. According to the Shockley-Ramo theorem (see equation 4.6), it only contributes a fraction of its charge to the signal, while it was drifting towards the electrode. Such defect states are present even in unirradiated diamond, while they do not occur in unirradiated silicon.

In case of unirradiated diamond, such defect states are often, but not exclusively, introduced by grain boundaries. Their amount increases with the radiation dose the sensor is exposed to. In order to calculate the amount of the deposited charge, the influence of trapping has to be measured. A figure of merit to quantify the quality of charge collection of the diamond is the charge collection distance (CCD). It is defined as the mean distance an electron hole pair separates, before it is being trapped. It can be calculated using the mobility $\mu$ and lifetime $\tau$ of electrons and holes and the electric field $E$ via

$$
\mathrm{CCD}=\left(\mu_{e} \tau_{e}+\mu_{h} \tau_{h}\right) E .
$$




\section{Diamond Pixel Detectors}

As a result, the CCD increases with the applied electric field and for that reason high fields are needed to successfully operate a diamond detector. A more convenient definition is

$$
\mathrm{CCD}=\frac{Q}{Q_{0}} d
$$

with the collected charge $Q$, the initially deposited charge $Q_{0}$ and the sensor thickness $d$. This formula can be derived from equation 5.1 assuming that the ratio of measured charge to deposited charge equals the fractional distance the charge carriers drift in the sensor, namely

$$
\frac{Q}{Q_{0}}=\frac{l_{e}+l_{h}}{d}
$$

with $l_{e, h}$ being the distance an electron or hole drift, respectively. The distance is calculated via the lifetime and the velocity of the charge carrier, with the later being the product of mobility and applied electric field. This yields

$$
\begin{aligned}
\frac{l_{e}+l_{h}}{d} & =\frac{\mu_{e} \tau_{e}+\mu_{h} \tau_{h}}{d} E \\
\Rightarrow \frac{Q}{Q_{0}} d & =\left(\mu_{e} \tau_{e}+\mu_{h} \tau_{h}\right) E .
\end{aligned}
$$

The assumption in equation 5.3 is only valid, as long as the thickness of the sensor exceeds the drift length of the charge carriers. Accordingly, equation 5.2 can only be applied, if the sensor thickness is significantly larger than the CCD.

According to equation 5.1 an infinitely strong electrical field would result in an infinitely large CCD. This is not the case, as the increase of CCD with the increase of the electric field is limited by the saturation velocity of the charge carriers [60]. The velocity $v$ can be calculated via

$$
v=\frac{\mu_{0} E}{1+\frac{\mu_{0} E}{v_{s}}}
$$

with $\mu_{0}$ being the mobility at low electric fields, $E$ the electric field and $v_{s}$ the saturation velocity. As a result of the finite saturation velocity, the measured CCD saturates for high electric fields.

Another method to increase the CCD of a diamond, other than applying a higher electric field, is pumping the diamond sensor with a very active radioactive source. The irradiation from this source deposits charges in the sensor, which are trapped by the defect states. If such a defect is close to the middle of the band gap, its detrapping time is typically large. This means, that the state remains filled and cannot trap other charges. Consequently, this reduces the concentration of unfilled defect states and therefore increases the CCD. Exposing the diamond sensor to UV-light, depumps the sensor, i.e. the trapped charges are released from the traps. 


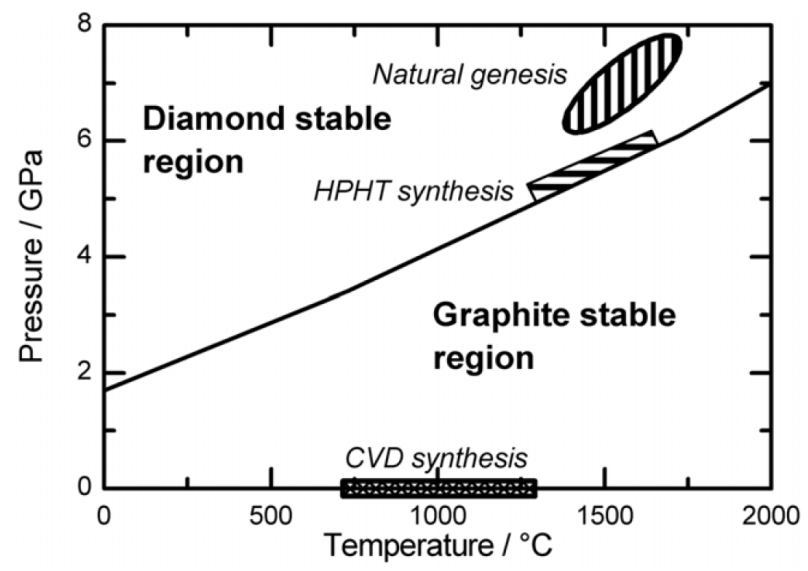

Figure 5.2.: Phase diagram of carbon. Regions of the different types of diamond formation are indicated [64].

\subsection{Production of artificial Diamonds}

Natural diamond is too expensive to use as a sensor material. Additionally, the shape of natural diamonds does most of the time not fit to the required specifications. Therefore, all the diamonds used as sensors are artificial diamonds. Historically, the first approach to manufacture artificial diamond, was to expose graphite to very high pressures and high temperatures (HPHT). In 1955, H. Liander and E. Lundblad reported the first successful production of diamonds using HPHT synthesis [61]. As the diamonds, which can be manufactured using this technique, are smaller than a millimetre in diameter, these are not suitable for tracking sensors. Consequently, the diamonds used as sensors are made via chemical vapour deposition (CVD).

Allegedly, the first CVD diamonds were already produced in 1952 by W. Eversole, but these results were not published until four years later [62]. Such diamonds are produced using a hydrocarbon plasma inside a reactor. The gas mixture typically consists of methane and hydrogen [63]. Its pressure can range from a few $\mathrm{kPa}$ to tens of $\mathrm{kPa}$, which is well below the threshold of natural diamond growth, as shown in figure 5.2. While at these conditions graphite is the preferred allotrope of carbon, the formation of diamond is possible, but at a much lower rate.

In order to begin the growth of diamond, the plasma has to be ignited. Several options are available, but the most common one is using microwaves. The very intensive microwaves can reach up to a power in the order of one $\mathrm{kW}$ and form a strong electromagnetic field inside the reactor. This causes the plasma to ignite. A scheme of such a reactor is shown in figure 5.3. The plasma is located only a few millimetres above a substrate, where the diamond film will be grown on. Inside the plasma, the hydrogen splits into free radicals, i.e. atomic hydrogen. These radicals remove one hydrogen atom 


\section{Diamond Pixel Detectors}

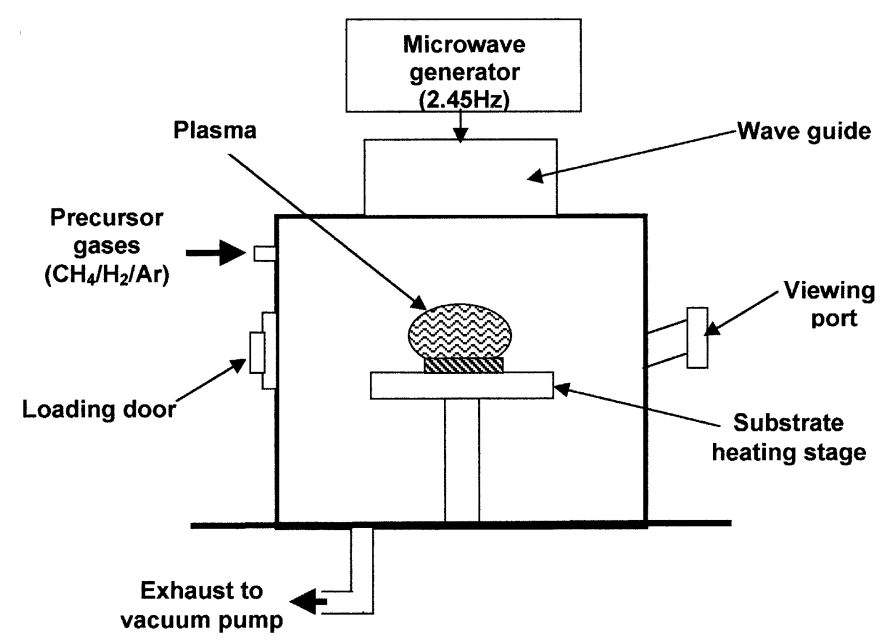

Figure 5.3.: Sketch of a microwave CVD reactor [65].

from the hydrocarbon gas, e.g. transforming $\mathrm{CH}_{x}$ to $\mathrm{CH}_{x-1}$, with $x=1-4$ [63]. The surface of the substrate also undergoes many collisions with the hydrogen radicals. In case of a single crystalline diamond substrate, it is hydrogen terminated, meaning that hydrogen atoms are attached to the open bonds of the carbon atoms at the surface. These hydrogen atoms can be removed by the collisions with the hydrogen atoms from the plasma, which leaves an open bond behind. A methane radical, e.g. $\mathrm{CH}_{2}$, can now use this open bond to form a carbon-carbon bond. In case of an $s p^{2}$ type bond, which is the preferred bond, the carbon atom can be etched away by the hydrogen radicals of the plasma. An $s p^{3}$ bond is formed less often, but is much more unlikely to be removed from the crystal. As a result, the crystal grows containing only $s p^{3}$ bonds.

The substrate used for the process has a large influence on the properties of the grown material. If a single crystalline diamond substrate is used, the resulting diamond will also be single crystalline (scCVD). The growth will start at random points of the substrate, but each of these grains has the same orientation, due to the orientation of the substrate. In case of a silicon substrate, the orientation of the diamond grains is not predefined, as silicon has the same lattice structure, but a larger lattice constant. The growth rate of these diamond grains depends on their orientation. Consequently, some grains grow faster than others and even outgrow them. This leads to an increase of grain size in the direction of total growth. A scheme of such a polycrystalline (pCVD) diamond is shown in figure 5.4.

For use as a sensor material, the density of the grain boundaries has a huge influence on the quality of the diamond. At grain boundaries, the crystal lattice is not perfect, but has a slight mismatch in the orientation between two grains. This results in some unbound electron states, which can act as charge traps. Consequently, a diamond with a lot of grain boundaries has a low CCD. For this reason, the diamonds are grown thicker 


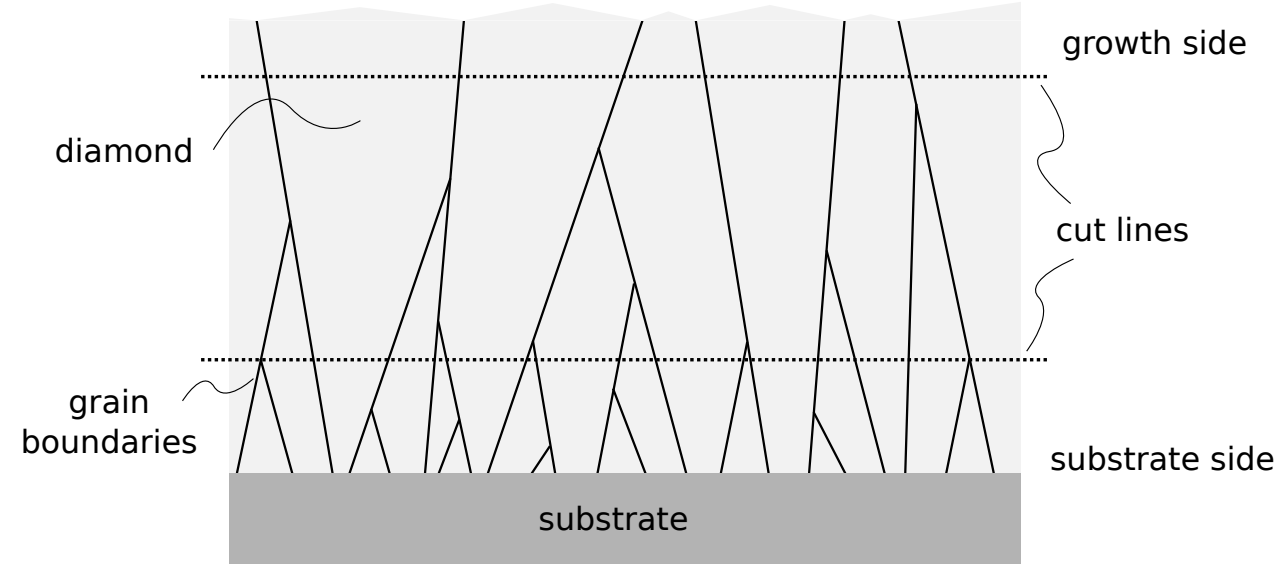

Figure 5.4.: Schematic cut through a pCVD diamond. The density of grains decreases in the direction of growth. Lines, where to cut the diamond to achieve the best possible quality, are indicated.

than needed, in order to remove the region near the substrate, where the density of grains is high. In order to distinguish between these sides they are referred to as substrate and growth side.

Due to the need of a single crystalline diamond substrate in order to grow scCVD diamonds, they are limited in size compared to pCVD diamonds, which can be grown on full silicon wafers. The high purity of scCVD diamond also demands low growth rates. These two factors combined lead to a significant pricing difference between scCVD and pCVD diamonds of the same size, with the former being more expensive.

\subsection{Radiation Damage and Radiation Tolerance}

The interactions of traversing particles with the sensor material do not always leave the crystallographic structure unaltered like e.g. the ionisation of an electron or the creation of an electron hole pair. Instead, some particles cause permanent damage to the material due to non-ionising energy loss (NIEL). According to the NIEL hypothesis, the total damage of the crystal scales with the fluence. This hypothesis has been tested numerous times for silicon and also for diamond [66,67]. The damage to the material is often done by removing an atom from the crystal lattice. Such a defect is called a vacancy. The displaced atom now resides between the regular sites of the lattice, a so called interstitial defect. Whether or not such defects occur depend on the sensor material, the type, and the energy of the traversing particle.

The minimal amount of energy needed to displace an atom depends on the crystal itself and the recoil direction. This energy threshold is two to three times higher than in 


\section{Diamond Pixel Detectors}

silicon (see table 5.1), which is advantageous. If the recoil energy is much higher than the minimal energy needed (e.g. $43 \mathrm{keV}$ for diamond), the displaced atom has enough energy to cause further displacements, creating a so called defect cluster [42]. At such energies, the incident particle still scatters elastically with the first displaced atom. If energies of a few hundred $\mathrm{MeV}$ are transferred, the particle scatters inelastically. This means that the atom of the crystal lattice is transmuted into a lighter atom which then scatters elastically with other atoms of the lattice grid. As a silicon atom is much heavier than a carbon atom, the possible transmuted cores are also heavier. These heavier cores cause a lot more damage to the grid than lighter cores, which explains the superior radiation tolerance of diamond compared to silicon for very high energetic radiation [67].

The actual amount of energy, which is transferred to the primary knock on atom depends on the energy and type of the incident particle. Charged particles, like e.g. electrons or protons, scatter via electromagnetic interaction with the atomic nucleus. Neutral particles, like a neutral pion, or heavy particles, like the proton, can scatter also via nuclear interaction with the nucleus [42]. The relative amount of energy loss due to ionisation, which does not result in permanent damage, and NIEL, which can cause permanent damage, does also depend on the energy of the incident particle. For charged particles with an energy above $10 \mathrm{MeV}$ almost all energy loss is due to ionisation [67]. At lower energies the fraction of energy loss due to NIEL increases significantly. As a result, low energetic particles have a higher potential of causing defects to the lattice. In order to compare the damage caused by the different types of radiation, their impact is normalised to the damage done by an incident neutron with a kinetic energy of $1 \mathrm{MeV}$.

Defects like a vacancy or an interstitial can introduce new energy states. These states can be in the energy gap between valence and conduction band and act as donors or acceptors. This changes the effective doping concentration of a sensor and in the case of silicon it can even change the effective sign of the space charge of the material. As radiation causes more p-type than n-type defects, a highly irradiated n-type sensor can change to a p-type-like sensor (e.g. see figure 4.4). For diamond, the introduced new energy states act predominantly as traps. Consequently, the CCD decreases with the total radiation fluence the sensor has been exposed to. This decrease can be quantified in terms of the mean free path $\lambda$ for electrons and holes [68]

$$
\frac{1}{\lambda}=\frac{1}{\lambda_{0}}+k \Phi .
$$

$\lambda_{0}$ is the mean free path before irradiation, $k$ the damage constant, and $\Phi$ the non ionising fluence. A decrease in the mean free path results in a decrease of CCD. As the formula describes the evolution of the mean free path, it can also be applied to silicon. The damage constant is a material specific quantity, i.e. it differs for silicon and diamond. However, it was measured that it does not depend on the type of diamond material, i.e. scCVD or pCVD diamond, as shown in figure 5.5 [69]. Both types of diamond material show the same behaviour as predicted by equation 5.7, with the same damage constant $k$. The difference between the two types of diamond lies in the 


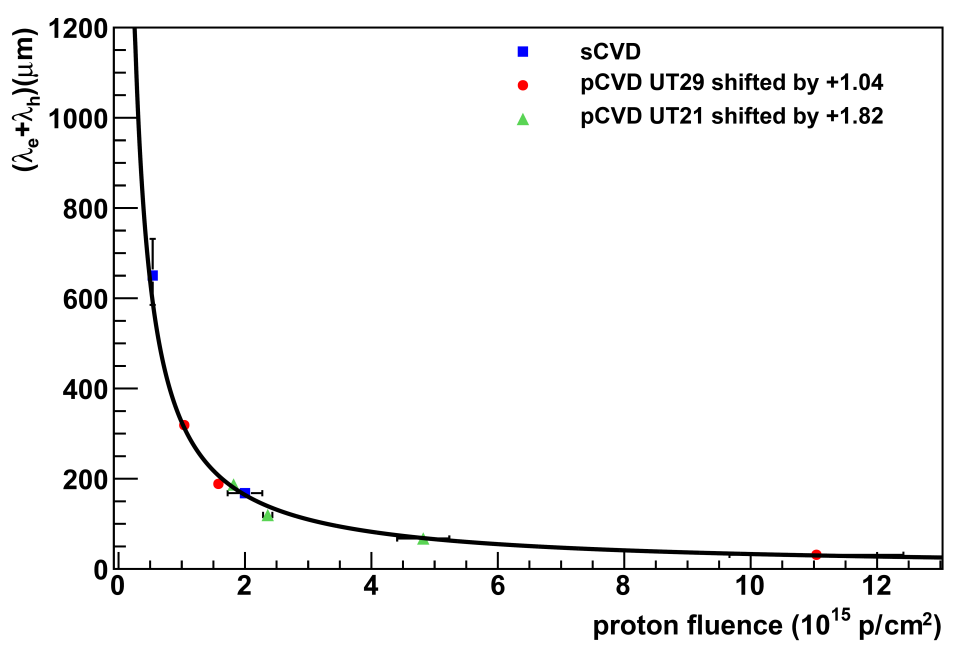

Figure 5.5.: Evolution of the combined mean free path of electrons and holes with respect to the accumulated fluence for different diamond samples [69]. The samples were irradiated with $25 \mathrm{MeV}$ protons. The values for the two pCVD samples have been shifted to a higher fluence of $1.04 \cdot 10^{15}$ protons $/ \mathrm{cm}^{2}$ and $1.82 \cdot 10^{15}$ protons $/ \mathrm{cm}^{2}$ for sample UT29 and UT21, respectively. This minimises the $\chi^{2}$ of a fit according to equation 5.7.

different values of the CCD for unirradiated samples. Generally, pCVD diamond start with a lower CCD than scCVD diamonds. In order to describe both types of diamond with one curve, pCVD samples are shifted to higher fluences so that the $\chi^{2}$ of the fit according to equation 5.7 is minimised. The shift indicates that the defects at grain boundaries can act as charge traps, with the same properties as the radiation induced traps. As the evolution of the mean free path is proportional to $\Phi^{-1}$, it decreases very rapidly for high quality samples. Therefore, the amount of charges collected after the exposure of the sensor to high radiation fluences does only slightly differ for high and low quality samples. Consequently, the use of high quality samples is no viable solution for a diamond sensor, which collects almost all charges even after being exposed to a high radiation fluence, as the CCD drops sharply.

With the known dependence of the mean free path on the fluence, the most probable value for the collected charge can be calculated [69], as shown in figure 5.6. In silicon much more charge is generated and initially also collected. However, after fluences of $10^{16}$ protons $/ \mathrm{cm}^{2}$ diamond collects at least the same amount of charges. The defects introduced by radiation into the silicon crystal do not only act as charge traps and as a result reduce the mean free path. As the band gap in silicon is very small, they can also act as donors or, more often, as acceptors. This increases the leakage current of the sensor and therefore the noise from the detector. Combining the increase of the noise and the decrease of the signal in silicon yields a significant decrease in signal to noise 


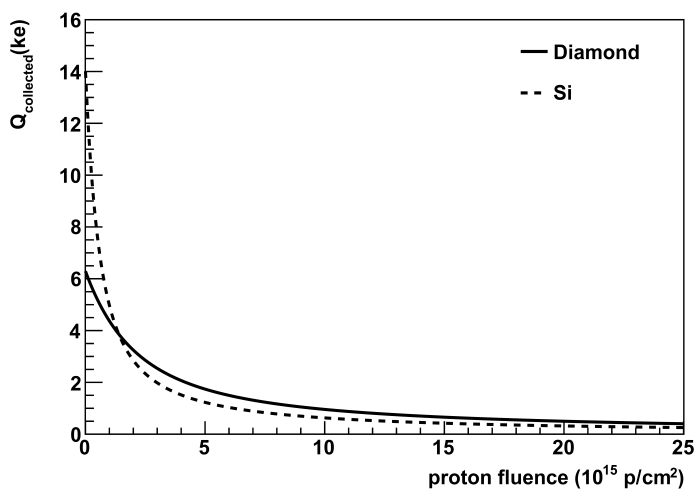

(a)

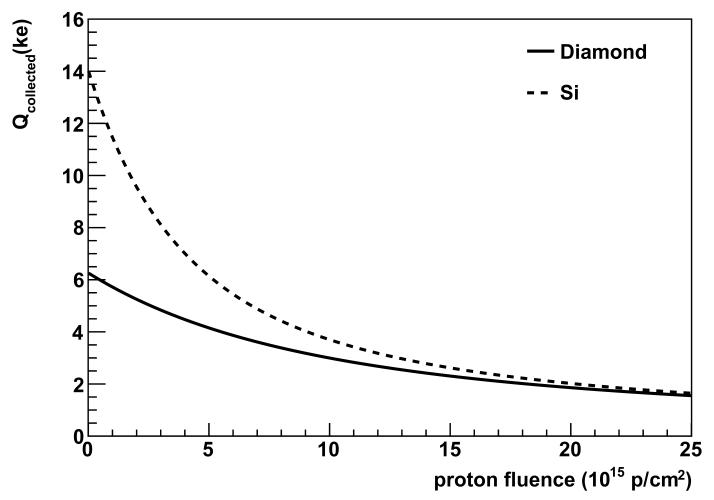

(b)

Figure 5.6.: Calculated most probable value for the amount of collected charge of a traversing $1 \mathrm{GeV}$ pion with respect to the accumulated fluence. The silicon and diamond sensor are both $200 \mu \mathrm{m}$ thick and were exposed to (a) $25 \mathrm{MeV}$ and (b) $24 \mathrm{GeV}$ protons [69].

ratio. Compared to diamond, the signal decreases too, but the leakage current stays negligible due to the large band gap [67]. Hence the signal to noise ratio is advantageous for diamond even at lower fluences, as shown in figure 5.7.

\subsection{Pixel Electrode Geometries}

As shown above, it is not enough to rely on high quality diamonds, in order to collect as many charges as possible even after a high irradiation dose. For such a high quality material, like scCVD diamond, the CCD decreases quite rapidly to a level of pCVD diamonds. Combined with the significantly higher costs of scCVD diamonds, other solutions are favoured.

With increasing radiation dose, the mean free path decreases. This decrease cannot be compensated by applying a higher electric field, as the operation voltage is already about $1 \mathrm{kV}$ and the velocity of the charge carriers saturates. Accordingly, in order to still collect all charges, the distance between the electrodes has to be reduced. Pixel sensors, diamond as well as silicon, typically have their electrodes on the surface, so called planar electrodes. Therefore, a reduction of the distance between the electrodes means thinning the sensor. But this also reduces the amount of deposited energy by traversing particles. Additionally, thinning a diamond sensor of the size of e.g. $2 \mathrm{~cm}^{2}$ from $500 \mu \mathrm{m}$ to $400 \mu \mathrm{m}$ is very delicate and can break the diamond [59].

An alternative are electrodes within the sensor bulk, as proposed by Parker et. al. in 1997 [70]. A schematic comparison of these so called 3D electrodes with planar electrodes 


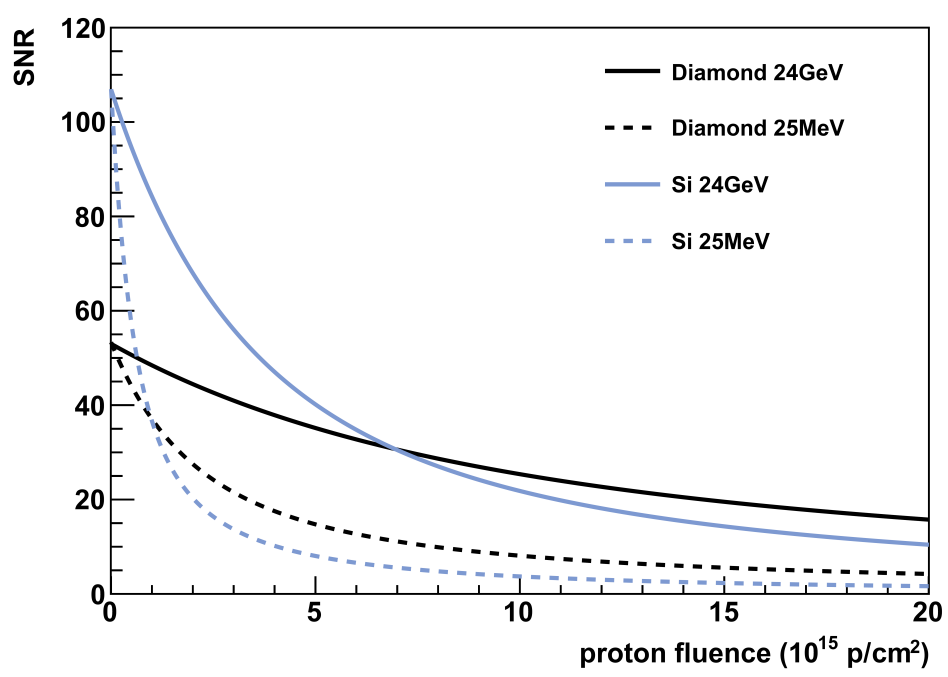

Figure 5.7.: Calculated signal to noise ratio (SNR) for diamond and silicon for a traversing $1 \mathrm{GeV}$ pion with respect to the accumulated fluence. The silicon and diamond sensor are both $200 \mu \mathrm{m}$ thick and have been exposed to $25 \mathrm{MeV}$ (dashed) and $24 \mathrm{GeV}$ (solid) protons [69].

is shown in figure 5.8. An advantage of this geometry is that it decouples the spacing between the electrodes from the thickness of the sensor. Consequently, the distance between the electrodes can be lower than the CCD, while still using a relatively thick sensor with a large deposited signal. As the geometry of the electric field changes to a more radial geometry and the distance between two opposing electrodes is reduced, a lower applied voltage is needed for the same strength of the electric field. The higher segmentation also reduces the time needed by the charges to drift to the electrodes and therefore the total collection time. Due to all these advantages, the 3D electrodes allow the use of low quality pCVD diamonds as sensors.

For silicon sensors, this technique has been established in the past years. Testbeam results show that they achieve a comparable performance with respect to the newest generation of planar pixel sensors [54]. Accordingly, 25\% of the IBL consists of 3D silicon sensors [71]. These come in two different flavours, differing in the depth of the electrodes in the sensor. The electrodes can either be etched from one side fully through the sensor or from both sides only partially through the sensor. This influences the handling of the sensors and the number of production steps.

An example of a possible layout of a 3D pixel cell is shown in figure 5.9. In this case one pixel cell consists of two electrodes which collect charges and six field electrodes. The latter are shared with neighbouring pixel cells, so that in total two field and two charge collecting electrodes in the diamond bulk per pixel cell have to be produced. It is possible to contact the field electrodes from the same side as the charge collecting 


\section{Diamond Pixel Detectors}
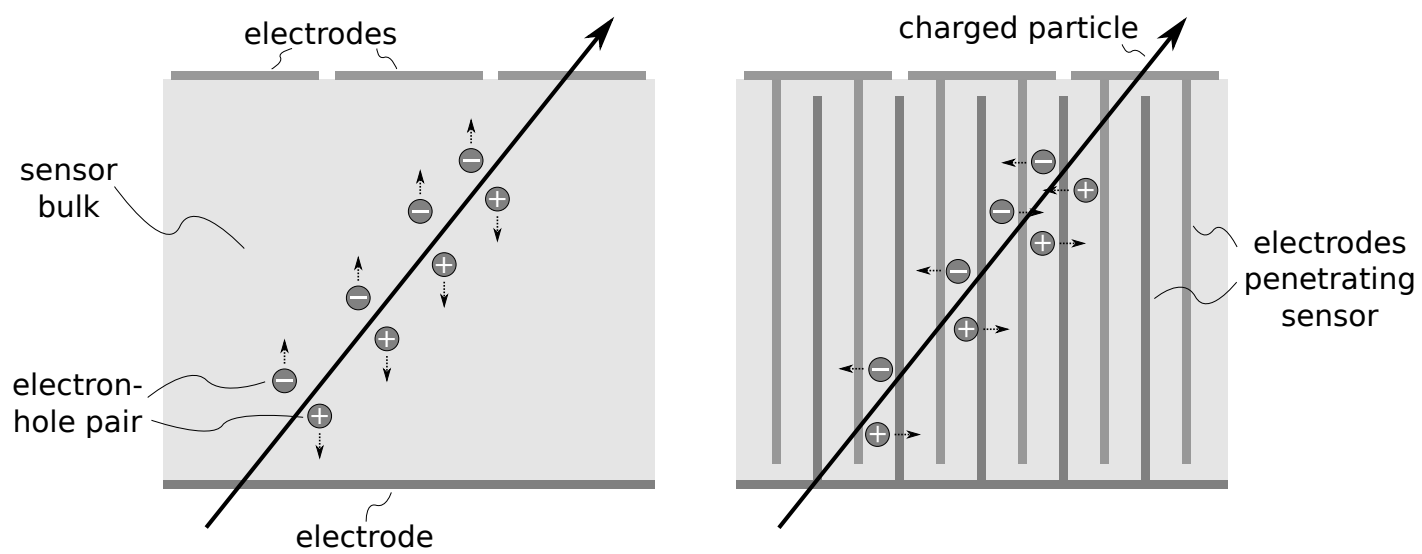

Figure 5.8.: Schematic cut through a sensor with planar (left) and 3D (right) electrodes. The direction of charge drift is indicated. Note that the diameter of the 3D electrodes and their spacing are not to scale.

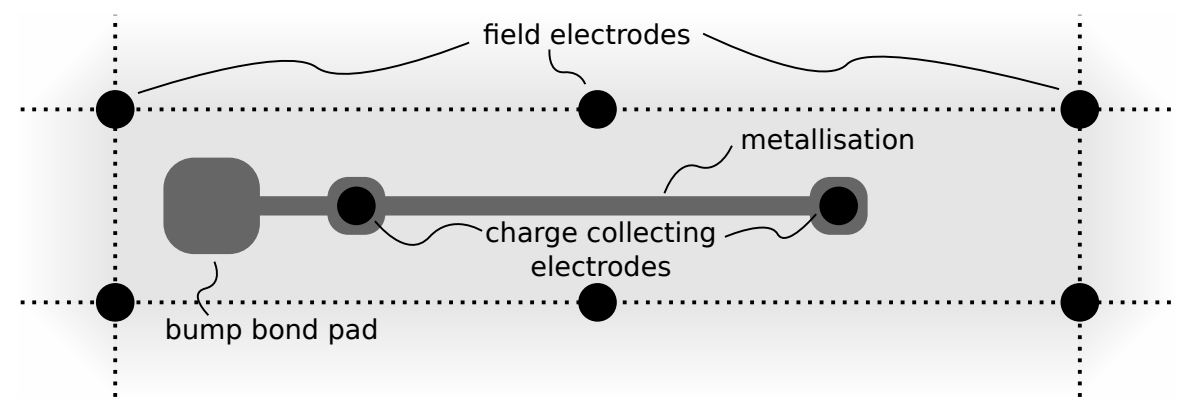

Figure 5.9.: Top view of a possible layout of one pixel cell. The size of one pixel is indicated by the dotted line. One pixel consists of two charge collecting electrodes and six field electrodes, which are shared with neighbouring pixel cells. A metal trace connects the two charge collecting electrodes. The field electrodes in this example are connected on the backside with each other via a trace. But it is also possible to connect them on this side. 
electrodes as long as the applied bias voltage is not too high, so that sparks between the electrodes occur. This option simplifies the connections of the sensor.

An advantage of diamond with respect to silicon is the fabrication of the electrode on the surface. In case of diamond this electrode consists of two metal layers, which are evaporated onto the surface ${ }^{1}$. For silicon sensors the electrode also consists of a metal layer. But like planar silicon sensors a doping with the opposed dopant around the electrode is needed in order to prevent shortening of pixels [54]. This increases the number of production steps and the complexity of the procedure.

3D electrodes minimise the inactive area due to guard rings with respect to planar electrodes, as the applied voltage is lower. Due to the high resistivity of diamond this area is even further reduced. It might even be possible that no guard rings are needed at all.

\subsection{Production of Electrodes inside the Diamond Bulk}

For 3D silicon sensors, the electrodes are produced by etching holes in the sensor bulk and filling them with appropriate electrode material [54]. This technique cannot be easily copied for diamond sensors. Diamond is chemically inert and as a consequence it cannot not be wet etched [72]. Using reactive ion etching is possible, but etch rates are fairly low (typically in the order of $200 \mathrm{~nm} / \mathrm{min}$ ) [72,73]. Alternatives like inductively coupled plasma and electron cyclotron resonance plasma etching are available, but are, at the moment, limited to a few micrometer depth [74-76].

Instead of drilling holes into the diamond and filling them with conductive material, another approach is to exploit the carbon nature of diamond. Converting the $s p^{3}$ bonded carbon atoms in diamond to $s p^{2}$ bonded atoms would yield a conductive connection. Femtosecond lasers can be used to induce such a phase change [77]. These lasers can reach a very high power for each pulse, reaching up to petawatts per pulse [78].

The typical range of available wavelengths of femtosecond lasers is from around $200 \mathrm{~nm}$ up to a bit more than $1000 \mathrm{~nm}$ [79]. For these wavelengths diamond is transparent [80], electrons cannot be directly ionised or even excited to the conduction band at these wavelengths. However, with the high fluence of photons accessible using a femtosecond laser, processes like multiphoton ionisation are possible [81]. This process, predicted in 1931 by Göppert-Mayer, is the simultaneous absorption of two or more photons by an electron and is only likely to happen at very high photon fluences [82]. It allows the excitation of electrons to the conduction band and eventually the ionisation the carbon atom. In order to understand the process of the phase change, it has to be differentiated between a phase change in pure diamond and a phase change at an already phase

\footnotetext{
${ }^{1}$ See section 5.6
} 


\section{Diamond Pixel Detectors}

changed seed layer.

For the initial phase change in pure diamond, the multiphoton ionisation is essential. The excited electrons collide with the atoms of the diamond lattice, effectively heating it up. Once a threshold energy $T_{g}$ is reached, the carbon atoms have enough energy to change their bonding from $s p^{3}$ to $s p^{2}$, which has a lower binding energy [79]. The threshold temperature depends highly on the environmental conditions. For example, at normal pressure and atmospheric air values of $T_{g} \approx 1000 \mathrm{~K}$ and $T_{g}<1370 \mathrm{~K}$ have been reported $[83,84]$. However, these are values measured for the phase change of a whole diamond surface. The threshold temperature for the phase change of individual atoms could be lower [85]. The excited electrons gain even more energy by inverse bremsstrahlung. In this process, the free electrons absorb photons while colliding elastically with lattice atoms in order to conserve the total momentum [86]. Depending on the parameters of the laser, i.e. power and pulse duration, the excited electrons can heat up the crystal lattice to the threshold temperature. For a low power and long pulse duration, the electrons stay in thermal equilibrium with the lattice. For short pulses with a high intensity, the electrons gain sufficient energy to ionise the atoms via direct impact, which creates an electron avalanche. Eventually, the temperature of the crystal lattice increases above the threshold energy [79].

As the density of the phase changed material is lower than for diamond, this initial phase change is more likely to happen in regions where some free volume is available [79]. This can be for example at grain boundaries or at the surface of the sample [87]. This hypothesis is supported by the observation of an incubation effect [88]. It was determined by measuring the transmittance of a diamond sample with respect to the number of pulses. For low fluencies, where no spontaneous phase change is possible, the transmittance does not immediately change, but sometimes it decreases only after tens or even thousands of pulses. This hints at the generation of stable micro defects in the sample, which locally create some free space. These cannot be observed by a decrease of the transmittance, but lower the threshold for the phase change.

The direct comparison of the minimal energy density needed to achieve the phase transition shows that the threshold on the surface is significantly lower [80]. While a phase change at the surface is observed with a pulse duration of $130 \mathrm{fs}$ at a minimal energy density of approximately $1 \mathrm{~J} / \mathrm{cm}^{2}$, a phase change in the bulk needs at least $5 \mathrm{~J} / \mathrm{cm}^{2}$. The threshold for a phase change of the bulk also only slightly depends on the duration of the pulse. It increases to approximately $8 \mathrm{~J} / \mathrm{cm}^{2}$ and $10 \mathrm{~J} / \mathrm{cm}^{2}$ for a pulse duration of $1 \mathrm{ps}$ and $3 \mathrm{ps}$, respectively.

This threshold is even lower, if some phase changed material is already present in the diamond [77]. Illuminating a pure diamond bulk with a sufficiently intens laser beam results in the formation of a discrete and continuous area of phase change. This is illustrated in figure 5.10. It shows that the phase change happens upstream of the focal 


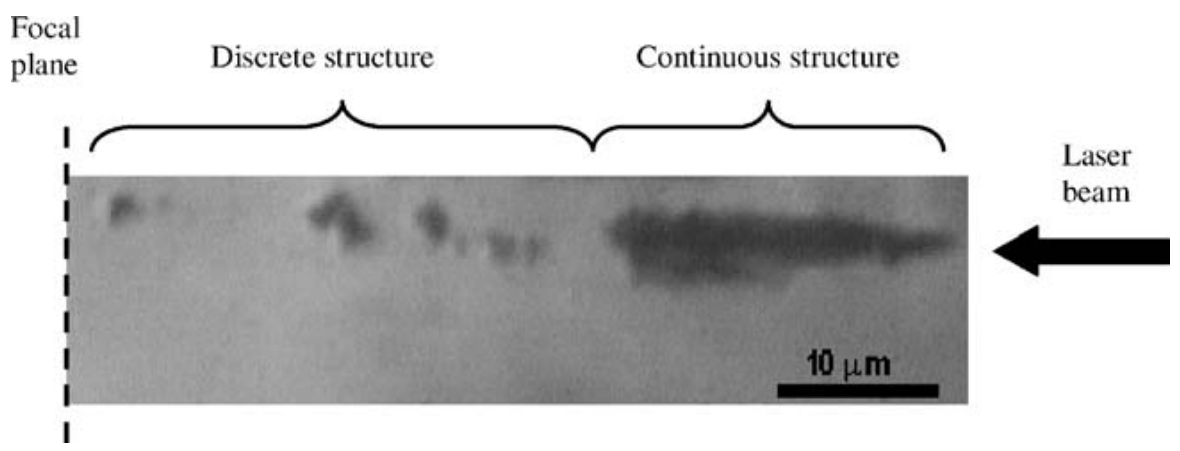

Figure 5.10.: Side view of a phase changed area (dark spots) inside a diamond. The direction of the laser beam and the position of the focal plane are indicated. Upstream of the focal plane the fluence decreases and different structures depending on the fluence can be observed [77].

plane. In regions near the focal plane, where the fluence is still high, isolated spots of phase changed material can be observed. As the picture was not taken after one, but several hundred pulses, it can be assumed, that the first spot which was created is the one nearest to the focal plane. At a later pulse the isolated spot more upstream was induced, as the fluence at that point is high enough to cause spontaneous phase change. This spot is optically dense for the laser and as a result the spot more downstream is conserved. This process repeats further upstream as long as the fluence is sufficient to cause spontaneous phase change. If this is not any more the case, the most upstream phase change spot grows continuously upstream. The growth stops once the fluence is too low even for continuous growth.

The lower energy density threshold of the continuous growth can be explained by the absorption of the laser beam by the phase changed material. Two hypotheses were proposed to describe the process in detail [77]. The first one assumes that the diamond layer surrounding the phase changed material thermally changes its phase. As the laser irradiation is completely absorbed in about $50 \mathrm{~nm}$ inside of the phase changed material, it heats up to temperatures around $100 \mathrm{eV}$. This heat dissipates equally from the source, resulting in about half of the energy being transmitted to the diamond layer. The diamond is then hot enough to undergo a phase transformation.

The second hypothesis revolves around the electrons in the phase changed material. As this material is $s p^{2}$ bound, some electrons need no additional energy to switch from the valence to the conduction band. These electrons absorb the laser beam and can reach very high velocities. As an example, assuming a laser wavelength of $1043 \mathrm{~nm}$ a single photon transfers an energy of $E_{\gamma}=h c \lambda^{-1} \approx 1.2 \mathrm{eV}$ to one electron. The electron then has an velocity of

$$
v_{e} \approx \sqrt{\frac{2 h c}{\lambda m_{e}}} \approx 6.5 \times 10^{7} \frac{\mathrm{cm}}{\mathrm{s}}
$$




\section{Diamond Pixel Detectors}

with $m_{e}$ being the mass of the electron. At these velocities the electrons move in the ballistic regime, i.e. they do not scatter with a large angle [79]. Consequently, the distance the electrons penetrate the diamond within a $290 \mathrm{fs}$ laser pulse is approximately $190 \mathrm{~nm}$. This triggers an optical breakdown of the diamond layer, meaning the hot electrons absorb the laser. These electrons collide with the lattice atoms, which eventually leads to a phase change [77].

Despite the incubation effect due to micro defects, each laser pulse can be considered as an isolated event. The pulses heat up the diamond locally above the threshold for a phase change, but due to the high thermal conductivity, the temperature decreases between the pulses, so that no significant heat from the previous pulse is noticeable. The time $t$ needed to spread the heat in a heat wave can be calculated via [89]

$$
t=\frac{E \cdot l}{\lambda \cdot A \cdot \Delta T}
$$

with $E$ being the energy deposited by one laser shot, $l$ the distance the heat wave travels, $\lambda$ the thermal conductivity of diamond, $A$ the surface area the heat flow crosses and $\Delta T$ the temperature difference. A typical value for the power of the laser used for this thesis is $100 \mathrm{~mW}$. With a pulse frequency of $200 \mathrm{kHz}$ this yields an energy of $E=500 \mu \mathrm{J}$ for a single shot. As calculated above, within one shot the electrons travel up to $190 \mathrm{~nm}$ inside the diamond ${ }^{2}$. Assuming a half sphere ${ }^{3}$ of this diameter yields a surface area of $A \approx 270 \times 10^{-15} \mathrm{~m}^{2}$. As stated above, a single laser pulse can heat up the material to temperatures up to $100 \mathrm{eV}$, which translates to $T \approx 10^{8} \mathrm{~K}$. With the diamond sample at room temperature of $T_{\text {cold }}=300 \mathrm{~K}, \Delta T=T$ can be approximated. The propagation length $l$ of the heat wave is defined as the distance at which the surface $A_{\text {cold }}$ of the resulting half sphere is that large, that the temperature is back to room temperature. The size of this sphere can be calculated via $A \cdot T=A_{\text {cold }} \cdot T_{\text {cold }}$. This yields a surface area of $A_{\text {cold }}=90 \times 10^{-9} \mathrm{~m}^{2}$. The resulting radius of this half sphere is $l=120 \mu \mathrm{m}$. Finally, the thermal conductivity of diamond is $2200 \mathrm{Wm}^{-1} \mathrm{~K}^{-1}$ [90]. Filling all these values into equation 5.9 gives $t \approx 1 \mu \mathrm{s}$. Although this is only a quick calculation and every simplification done increases the time the heat wave needs to propagate, the result is still significantly lower than the time between two laser pulses, which is $5 \mu \mathrm{s}$. Consequently, the laser pulses can be viewed as individual events.

In order to grow long continuous channels of phase changed, conductive material, the fluence of the laser beam has to be below the threshold for spontaneous phase change inside the diamond bulk and above the threshold for continuous growth. This can be achieved by setting the fluence in the focal area inside this parameter range. This of

\footnotetext{
${ }^{2}$ This assumes a point like source, which is not the case. Instead the electrons are excited from the tip of the phase changed material, which has a non-zero diameter. This would make the surface area even larger.

${ }^{3}$ The other half of the sphere consists of already phase changed material, which does not have a thermal conductivity as good as diamond. For this reason it is assumed, that the heat only dissipates into the diamond.
} 
course demands a seed for the phase change inside the bulk. Luckily, the threshold for surface phase change is in the same order as for exclusively continuous growth inside the diamond bulk [79]. Consequently, the technique to grow continuous channels is to move the focal plane through the diamond, starting at the downstream surface of the sample, which in this thesis will be referred to as the seed side. Using this technique, channels of $670 \mu \mathrm{m}$ length were reported [91]. Depending on the movement of the focal area inside the diamond, also three dimensional structures are possible [92].

Experimentally, the easiest way to move the focal plane with respect to the diamond is to move the sample. Besides the fluence per pulse, the speed of this movement along the beam axis is an important parameter for the growth of phase changed, conductive channels. Although the diameter of the channels depends more on the fluence, it is also correlated with the velocity of the sample [92]. A lower velocity yields a smaller diameter. This can be understood by assuming the generation of micro defects. If the focal plane moves very slowly through the diamond, the distance the focal plane moves between two pulses is smaller than the area of phase change triggered by one pulse with sufficient fluence. Accordingly, the phase change always happens very upstream, where the fluence threshold for phase change is barely reached. As the movement of the focal plane is so slow, the same interface between diamond and phase changed material is illuminated repeatedly before the fluence is high enough to trigger a phase change. However, the creation of stable micro defects is possible even at such a low fluence. If enough micro defects are accumulated, the threshold for phase change is decreased. As a result, regions with the highest density of micro defects are the first to change their phase. These regions are located at the very centre of the beam axis. Such a small phase changed area is enough to absorb the beam and prevent additional phase change more downstream. Consequently, lower velocities of the sample yield a slightly smaller diameter of the channels.

In order to use such channels as electrodes, the shape of the channel inside the diamond bulk, the shape at the diamond surface, and the conductivity are important. As the channels should collect charges the field should be as uniform as possible. This means that the surface of the channels inside the diamond bulk should be as smooth as possible.

The shape at the surface is critical with respect to the contact via a metal electrode (see section 5.6). If the interface between the conductive channel and the metal is properly established, charges can be collected with the channel. Depending on the growth direction of the channel, the structures at the surface differ significantly. On the seed side, the phase changed material is only exposed once to the laser beam. Once the material changes its phase, it blocks the laser beam and prevents further illumination of the surface. As the density of the transformed material is lower than for diamond, a small bump of phase changed material can be observed [93].

This situation is different for the side where the focal plane leaves the diamond, the 


\section{Diamond Pixel Detectors}

so called exit side. In this case, no further material can block the laser beam once the exit side has undergone a phase transformation. This leads to a repeated absorption of the laser beam on the surface, which heats up the material and eventually evaporates parts of it. Consequently, crater like structures are created [94].

In order to collect charges, the channels have to be conductive. But their resistance also has an influence on the thermal noise from the sensor. The current $i_{n}$ of an ideal resistor $R$, which is as large as the thermally generated noise of that resistance is given via $[42]$

$$
\frac{\mathrm{d}\left\langle i_{n}^{2}\right\rangle}{\mathrm{d} f}=\frac{4 k T}{R}
$$

with $k$ being the Boltzman constant, $T$ the temperature and $f$ the frequency of the noise current. The frequency spectrum of this noise equals the spectrum for shot noise, which is caused by the discrete nature of the elementary charge. This can be calculated via

$$
\frac{\mathrm{d}\left\langle i_{n}^{2}\right\rangle}{\mathrm{d} f}=2 I q
$$

where $I$ is the current which causes the noise and $q$ the charge of the charge carriers, hence in this case the elementary charge. Combining equations 5.10 and 5.11 , the current of the resistance of the electrode which is as large as the thermally generated noise can be calculated:

$$
I=\frac{2 k T}{q R} .
$$

Assuming a resistance of $R=1 \mathrm{M} \Omega$ for the electrode and room temperature $(T=300 \mathrm{~K})$ this yields that currents lower than $I \approx 50 \mathrm{nA}$ can not be detected due to the thermal noise.

This current has to be compared to the current induced by the drifting charges. In a $500 \mu \mathrm{m}$ thick diamond sample, a mip deposits around $Q=18,000 \mathrm{e}$. Assuming a drift velocity of $5 \times 10^{6} \mathrm{~cm} / \mathrm{s}$ [58] and a maximum distance to the nearest collecting electrode of $75 \mu \mathrm{m}$ these charges would be collected within $t=1.5 \mathrm{~ns}$. Accordingly, the resulting current would be $I_{\text {coll }}=Q / t \approx 2 \mathrm{~mA}$. This is two orders of magnitudes higher than the noise from the electrode. Accordingly, the noise from the electrodes inside the diamond bulk is not too high to measure the deposited charge.

\subsection{Metallisation of Diamonds}

In case of planar electrodes, the interface between the diamond and the metal electrodes is very delicate. In order to achieve a good contact, the surface of the diamond has to be oxygen terminated [59]. The surface of the diamond should also be cleaned from any residual metal atoms or any other contamination. For this purpose, a cleaning recipe has been developed at Ohio State University, which in a final step uses oxygen plasma etching in order to terminate the diamond surface. This cleaning is crucial for planar 
diamond sensors, as in this case the charges are collected through the diamond metal interface. For samples with 3D electrodes, the charges should be predominantly collected by the electrodes inside the diamond bulk, so that they do not rely on a good diamond to metal contact. Nevertheless, this procedure is also applied to them. As some of the cleaning steps potentially remove phase changed material from the channels, this is done prior to the fabrication of the 3D electrodes.

The metal electrodes themselves typically consist of $50 \mathrm{~nm}$ of chromium with $100 \mathrm{~nm}$ gold on top. After a photo lithography process, which defines the geometrical structure of the planar electrodes, they are evaporated onto the sample. For this, the diamond is put in a vacuum vessel. Inside this vessel, a heat source evaporates the metal for the electrodes, which condensates on the diamond surface. In order to achieve a uniform coating, the diamond is rotated horizontally. After removing the diamond from the vessel, the photoresist is lifted off, which also removes all the metal on it. Only the metal on the diamond remains.

This method of electrode fabrication suffers from difficulties in coating steps with an aspect ratio of more than 0.5, i.e. a hole should not be deeper than half its diameter [95]. On the exit side of the diamond, crater like structures are present, which can have a higher aspect ratio. Assuming that on the walls of these craters only little conductive material is left, the metal of the electrode has to reach the bottom of the crater in order to establish a contact. 

CHAPTER 6

\section{D Electrodes in optical grade polycrystalline Diamond}

As shown in the previous chapter, 3D electrodes are a promising concept to use low grade pCVD diamond as a sensor material. In this chapter the production and measurements of the channel quality are discussed. Section 6.1 gives an overview of the utilised diamond samples and section 6.2 explains the setup of the femtosecond laser. First results of the phase change are discussed in section 6.3 and in section 6.4 the induced mechanical stress is determined. Not only straight channels of phase changed material are possible, but also tilted channels, which have two contacts on one side of the diamond, as shown in section 6.5. Finally, in section 6.6 the concept of Raman spectroscopy and measured spectra of phase changed material are discussed.

\subsection{Optical grade polycrystalline Diamond Samples}

The diamond samples used throughout this chapter are pCVD diamonds, which were purchased from Diamond Materials GmbH in Freiburg. In total four samples with equal properties were bought, of which three were used for this thesis. The types of measurements performed with each sample are summarised in table 6.1. A picture of a sample can be seen in figure 6.1. These are transparent and of optical grade ${ }^{1}$, which is the lowest grade suitable for radiation sensors. A lower grade generally means that the diamond has more grains and defects, which results in a lower CCD. These diamonds can be grown faster than higher grades, which reduces their price. Such diamonds are intended to be used e.g. as beam windows for lasers. The samples have a size of $5 \times 5 \mathrm{~mm}^{2}$ with polished front- and backsides. The vendor claims that the diamonds have a thickness of $500 \mu \mathrm{m}$ with a tolerance of $+100 \mu \mathrm{m}$. One sample has been measured to be $540 \pm 5 \mu \mathrm{m}$ thick.

\footnotetext{
${ }^{1}$ Higher grades are electronic grade and detector grade, with the latter having a higher CCD.
} 
6. 3D Electrodes in optical grade $p C V D$ Diamond

\begin{tabular}{l|ll}
\hline ID & Channels & Measurements \\
\hline Goe-pCVD-01 & two sets with variation of velocity and resistivity \\
& power & \\
Goe-pCVD-02 & variation of segmentation, channels not & induced stress, \\
& fully extending through the sample & Raman spectra \\
Goe-pCVD-03 & triangular channels, higher velocity & resistivity \\
\hline
\end{tabular}

Table 6.1.: List of the optical grade pCVD diamond samples used for this thesis. The main focus of the produced channels and the performed measurements are stated.

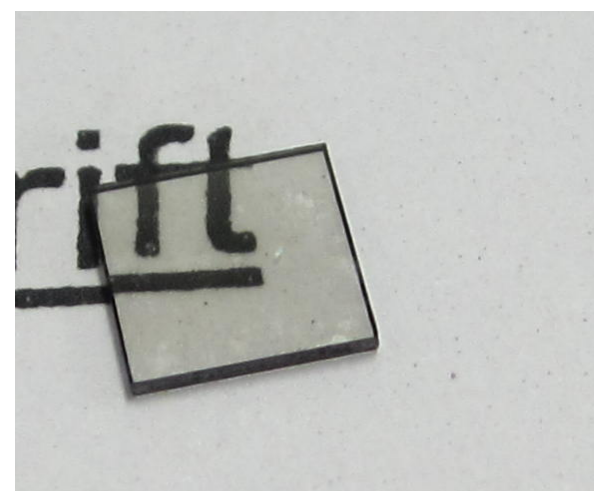

Figure 6.1.: Picture of a pCVD diamond sample from Diamond Materials GmbH in Freiburg. 


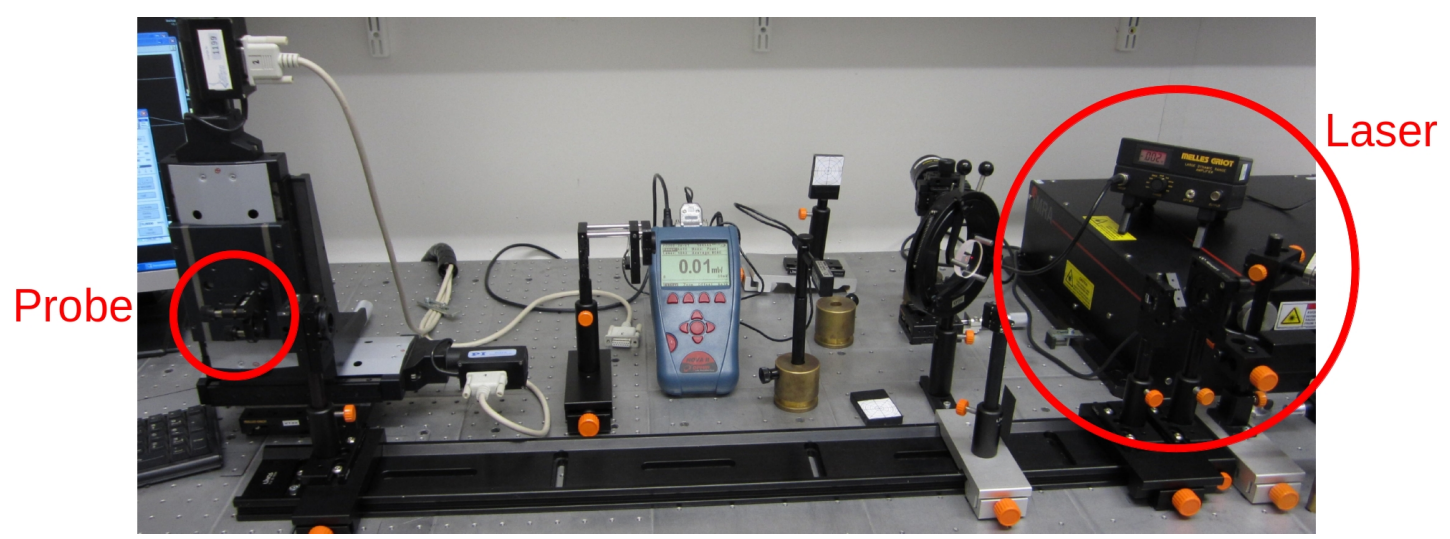

Figure 6.2.: Photograph of the setup at Laser-Laboratorium Göttingen e.V. The femtosecond laser is located on the right. The diamond sample is mounted on a motorised stage on the left. The power meter in the centre is used to measure the average power of the laser.

\subsection{Femtosecond Laser Setup}

For the production of all channels in this thesis, a femtosecond laser from Laser-Laboratorium Göttingen e.V. was used. A picture of the setup can be seen in figure 6.2. The laser itself is an FCAL $\mu$ Jewel D-400 ytterbium-doped fibre based laser from IMRA America. It provides pulses of $290 \mathrm{fs}$ length with a wavelength of $1043 \mathrm{~nm}$ and a repetition rate of $200 \mathrm{kHz}$. This rate is very high compared to other setups, which use lasers pulsed at $1 \mathrm{kHz}[77,88,94]$. The average power of the laser can reach up to $300 \mathrm{~mW}$, which equals a maximum pulse energy of $25 \mathrm{~mJ}$.

Figure 6.3 shows a schematic view of the setup. The diamond is mounted on a motorised, 3-dimensional, movable stage with its surface perpendicular to the laser beam. The positions of the axes are controlled via a PC. Their maximum velocity in each direction is $100 \mu \mathrm{m} / \mathrm{s}$. A lens directly in front of the sample focuses the beam, resulting in a focal region of $31.8 \mu \mathrm{m}$ in length and $4.6 \mu \mathrm{m}$ in diameter [96]. A shutter is used to block the laser beam when moving the diamond to the next channel. It is controlled by a PC within the same program, which moves the stage. The power of the laser beam is measured before and after the production of channels by moving a power meter into the beam. It is adjusted by an attenuator with an accuracy of $\pm 1 \mathrm{~mW}$.

In order to align the surface of the diamond with respect to the beam, the beam line is shared with a He-Ne-laser. Although the diamond is transparent for this laser beam, parts of it are reflected at the interface of air to diamond. The reflected light from the diamond is reflected by a semitransparent mirror, which guides the beam onto a power meter. A maximum in the intensity of the reflected light can be measured, when the focus is on the front surface of the diamond $[97,98]$. This maximum can be used to 


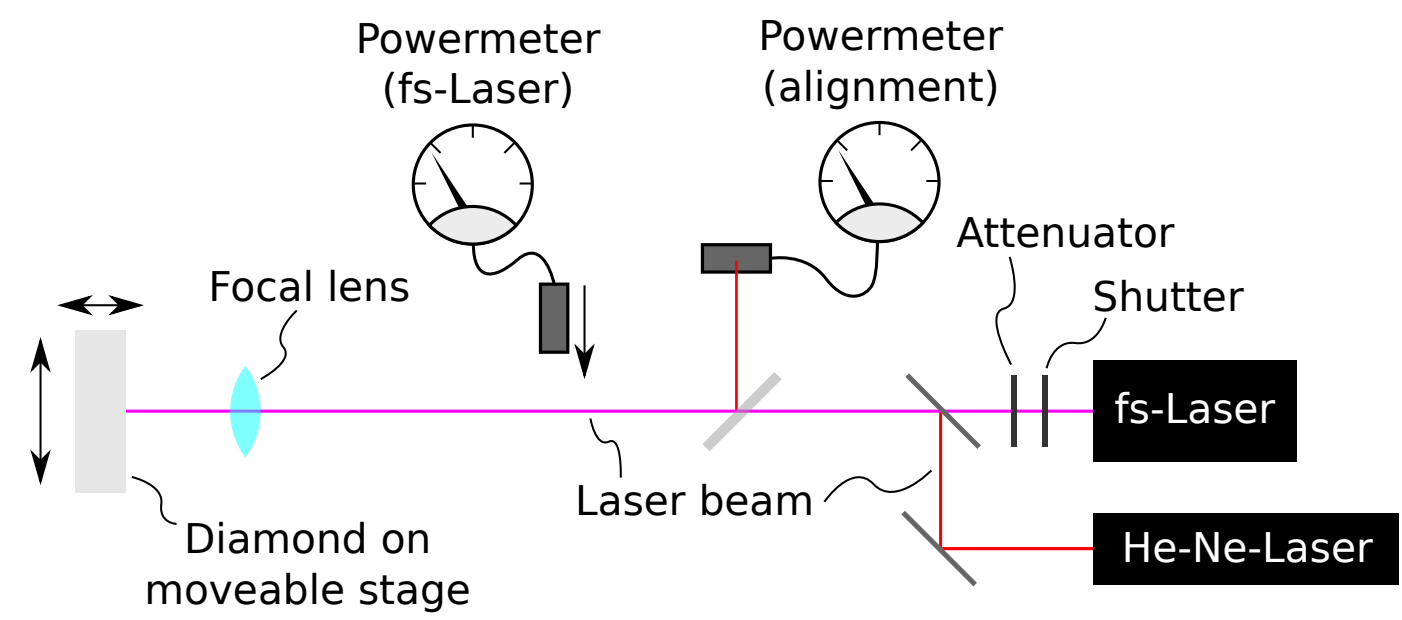

Figure 6.3.: Schematic view of the femtosecond laser setup. In order to align the moveable diamond sample, the beamline is shared with a He-Ne-laser.

measure and adjust the orientation of the surface with respect to the laser beam. A relative deviation of the surface with respect to being perfectly perpendicular to the beam of $5 \mu \mathrm{m}$ along a distance of $2 \mathrm{~mm}$ can be achieved, which equals $0.15^{\circ}$.

Before growing the channels, the diamond sample is moved, so that the focal area of the laser beam is behind the downstream surface of the sample. In order to produce a channel, the shutter is opened and the diamond is moved downstream until the focal area exits the diamond through the upstream surface. The shutter is closed and the diamond is moved upstream and perpendicular with respect to the beam to the position of the next channel. This process is repeated until all channels are produced. In order to ensure that the focal area is behind or in front of the diamond, respectively, an overdrive of $100 \mu \mathrm{m}$ to $150 \mu \mathrm{m}$ for each side is chosen.

\subsection{Observation of phase changed Material}

The first phase changed material was produced as a proof of concept with very few channels in Goe-pCVD-01. Dark channels inside the transparent diamond bulk could be visually observed. In order to study the dependence of the channel growth on the laser parameters, subsequently the velocity of the diamond and the power of the laser were varied for a second batch of channels. These two parameters are the only parameters which can be varied without changing the setup or the laser.

For the second batch of channels, values of $5,10,20,40,70 \mu \mathrm{m} / \mathrm{s}$ were chosen for the velocity of the diamond with respect to the beam. The power was set to $40,45,50,60,80$, 


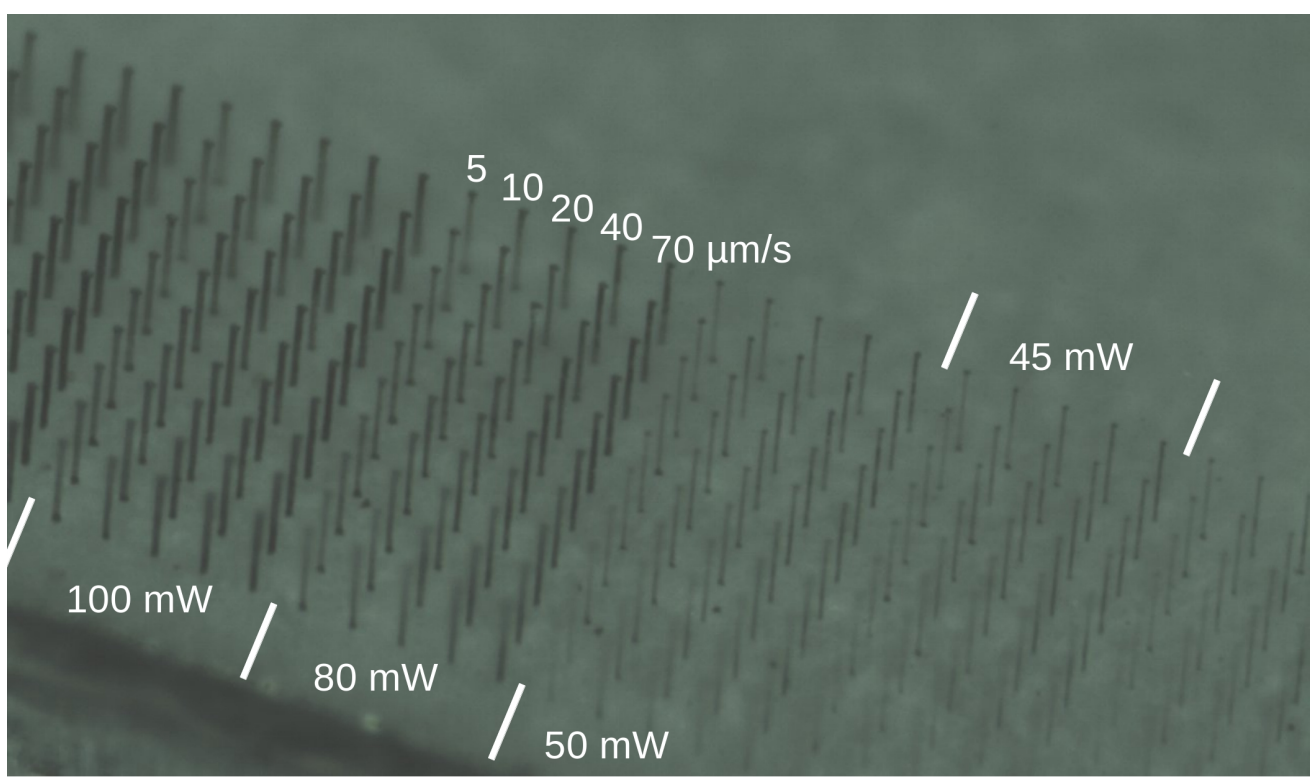

Figure 6.4.: Side view of channels produced in a pCVD diamond. The power of the laser and the velocity of the diamond sample are indicated. The order of the velocities is the same for all power settings. For all channels in a vertical line the same set of parameters was used.

$100,120 \mathrm{~mW}$. For each combination of these parameters ten channels were produced, totalling 350 channels. These are organised in a rectangular grid of $10 \times 35$ channels with a distance of $65 \mu \mathrm{m}$ to their nearest neighbour. A picture of some of these channels is shown in figure 6.4. For all channels, continuous unbroken pillars of phase changed material can be observed, stretching through the complete bulk of the sample. The diameter of the channels inside the diamond bulk varies with the power of the laser, as well as with the velocity of the sample. For higher powers an increase of the channel diameter inside the diamond can be observed. To a lesser degree, a lower velocity yields a lower diameter, especially for channels with a low power setting. This agrees with the behaviour of the channel diameter with respect to velocity and power as reported by Kononenko et. al. [92]. The exact change of the diameter with respect to the growth parameters is hard to quantify, as the resolution of the camera of the microscope is too low. The width of the channels is only between two and three pixels, which results in an upper limit of $18 \mu \mathrm{m}$ for their diameter.

In order to measure the resistivity of the channels, they have to be contacted. As the diameter of the channels at the surface is between $7 \mu \mathrm{m}$ and $14 \mu \mathrm{m}$, a direct contact with a probe needle is almost impossible to achieve. For this reason, metal electrodes have to be fabricated on the surface of the diamond. A mask was designed for this metallisation, which can be seen in figure 6.5. On the exit side, the metallisation consists of 350 


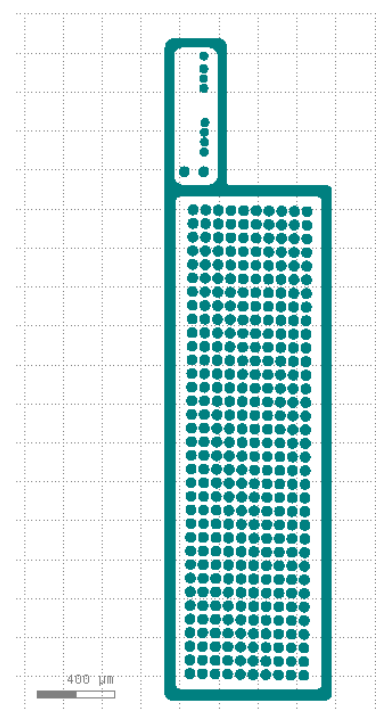

(a) Frontside

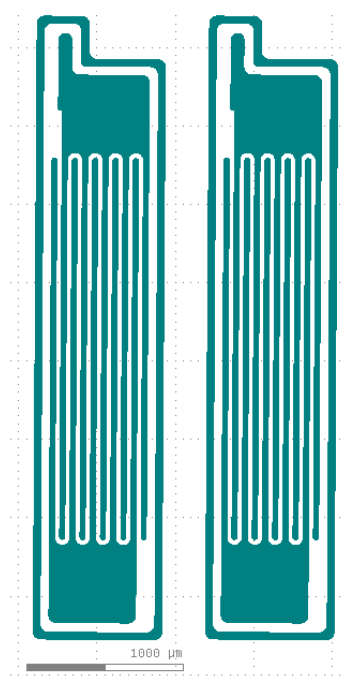

(b) Backside

Figure 6.5.: Masks used for the metallisation of Goe-pCVD-01. On the backside, the strip structure is copied to an area with no channels. This is done in order to measure the influence of the channels. Note the slightly different scales of front- and backside.

dots with a diameter of $50 \mu \mathrm{m}$. Additionally, the channels which were produced during the first test can also be contacted. On the seed side, the channels are connected via strip electrodes. In total ten strips span across the whole channel grid. The strips are alternately connected to large metallised areas at the top and at the bottom of the grid. The metallisation of this sample was done at Ohio State University (OSU) via vacuum evaporation [99]. In order to measure the resistivity of the channels, the seed side is connected via the two large pads of the strip electrodes and the exit side via a probe needle. For all channels the resistance is measured individually. The obtained values are in the order of the resistance of the diamond bulk and do not differ for channels with different growth parameters. Accordingly, these channels are not conductive [98].

The very high measured resistance of the channels can be caused by a very high resistance of the phase changed material. Another reason can be an insufficient contact between the phase changed material and the metal of the electrode. This might have originated from an insufficient cleaning of the sample prior to the production of the channels. For this reason, another batch of channels was produced in this sample, which was properly cleaned before metallisation. A cleaning of the diamond surface after the production of channels might etch some of the phase changed material. The new batch follows the same structure as the channel grid before, so that the metallisation mask could be reused. The same values were used again for the power and for the velocity values of $20,40,70 \mu \mathrm{m} / \mathrm{s}$ were chosen. For each pair of parameters, ten channels were 


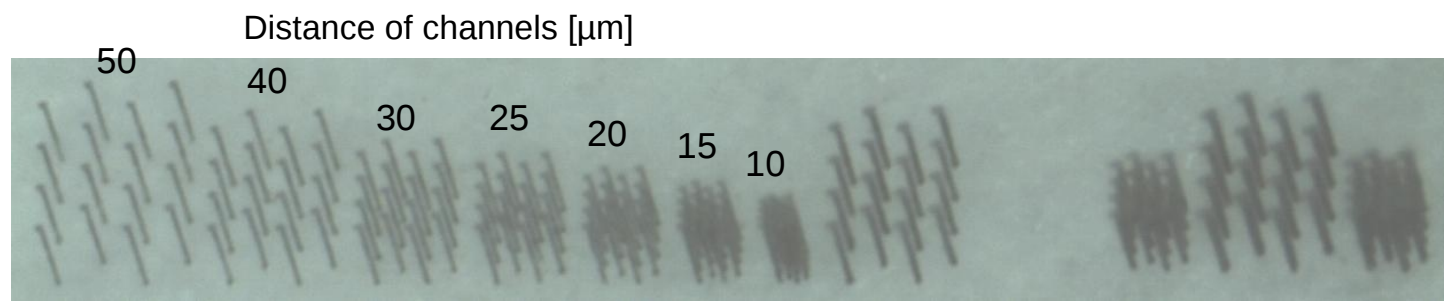

Figure 6.6.: Picture of channels intended for the measurement of the induced mechanical stress. For the left half, the spacing of the channels is indicated. These were grown with a laser power of $50 \mathrm{~mW}$ and a sample velocity of $50 \mu \mathrm{m} / \mathrm{s}$. Additionally, in the right half grids grown at $80 \mathrm{~mW}$ and $120 \mathrm{~mW}$ and distances between channels of $40 \mu \mathrm{m}$ and $20 \mu \mathrm{m}$, respectively, can be seen.

produced. Additionally, for a power of $46 \mathrm{~mW}$ in total 30 channels were grown under a nitrogen atmosphere. The reason for this is the assumption, that on the exit side the laser beam ignites a plasma and burns away parts of the phase changed material. In order to avoid this, the exit side was put under a nitrogen atmosphere. The sample was again metallised at OSU after removal of the metallisation from the first batch. Again, the measured conductivity for all channels was of the same order as for the diamond bulk.

\subsection{Measurement of channel induced Mechanical Stress}

The phase transformation changes for some atoms the bond configuration from $s p^{3}$ to $s p^{2}$, modifying the bond lengths. For diamond these are in every direction $152 \mathrm{pm}$. For graphite, which consists solely of $s p^{2}$ bonds, within a lattice plane the distance is only $142 \mathrm{pm}$, but between two crystal lattices the distance is $340 \mathrm{pm}$ [100]. This results in an overall higher density for diamond compared to graphite, which is $3.51 \mathrm{~g} / \mathrm{cm}^{3}$ and $2.26 \mathrm{~g} / \mathrm{cm}^{3}$, respectively [101]. As the phase changed material is not pure graphite, but more like amorphous graphite or diamond like carbon, the density of it may be in between these two values. Nevertheless, it is lower than for the surrounding diamond, which will induce mechanical stress in the material.

This stress could be critical for the stability of the sensor, so the induced stress has to be measured. In order to do this, a second pCVD diamond sample, Goe-pCVD-02, was used. In this sample grids of channels were produced with constant parameters for power of the laser and velocity of the sample, but with varying distances between the channels. One grid consists of four by four channels. Every second row of channels is staggered by half the channel spacing. This resembles a more realistic electrode configuration for read out and biasing channels, than a rectangular grid. A picture of the resulting channels is shown in figure 6.6. In table 6.2 the settings used to grow the channels are summarised. 
6. $3 D$ Electrodes in optical grade $p C V D$ Diamond

\begin{tabular}{rr|r}
\hline Laser power $[\mathrm{mW}]$ & Sample velocity $[\mu \mathrm{m} / \mathrm{s}]$ & Distance between channels $[\mu \mathrm{m}]$ \\
\hline 50 & 50 & 50 \\
50 & 50 & 40 \\
50 & 50 & 30 \\
50 & 50 & 25 \\
50 & 50 & 20 \\
50 & 50 & 15 \\
50 & 50 & 10 \\
\hline 80 & 50 & 40 \\
80 & 50 & 20 \\
\hline 120 & 50 & 40 \\
120 & 50 & 20 \\
\hline
\end{tabular}

Table 6.2.: Settings used to grow the channels in the four by four grid in order to measure the induced stress.

Out of the 176 produced channels, 21 do not extend fully through the diamond, as seen by optical inspection. This yields a success rate of $(88 \pm 2) \%$. This rate depends on the spacing of the channels. For channels with a segmentation of $25 \mu \mathrm{m}$ or more the success rate is $(95 \pm 3) \%$, for channels with a tighter spacing $(77 \pm 6) \%$ at a laser power of $50 \mathrm{~mW}$ and a sample velocity of $50 \mu \mathrm{m} / \mathrm{s}$. This indicates that stress is induced around the channels, which alters the threshold for phase change.

The influence of neighbouring channels on the growth of new channels can be very well observed at the grid grown with a laser power of $120 \mathrm{~mW}$ and a spacing of $20 \mu \mathrm{m}$. A picture of the seed side is shown in figure 6.7. The channels have a diameter of approximately $10 \mu \mathrm{m}$. In the picture the channels were produced in horizontal rows from top right to bottom left. As it can be seen, the first channel starts inside the diamond and does not reach the seed side. The shape of the other channels is not circular as expected but more bean-shaped. It shows a clear indentation in the direction of the first channel. This shape is a strong indication that the induced mechanical stress has an influence on the production of channels and that the production of very tightly spaced channels has to be done carefully.

The induced mechanical stress in the diamond sample is visualised using crossed polarisers. These linear polarisers are placed above and below the diamond sample, with the light through the diamond being parallel to the channels. The polarisers are either orientated parallel or perpendicular to each other. A schematic view of this setup is shown in figure 6.8. With this method the tension perpendicular to the propagation direction of the light can be measured [102]. This tension can be described by two modules 


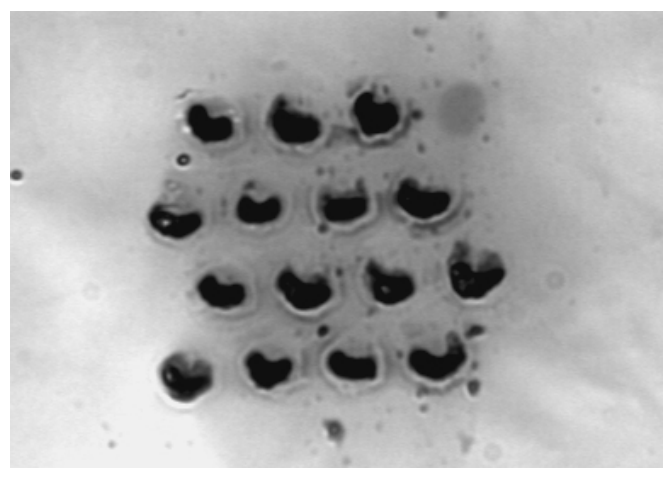

Figure 6.7.: Picture of the seed side for channels produced with a laser power of $120 \mathrm{~mW}$, a sample velocity of $50 \mu \mathrm{m} / \mathrm{s}$, and a spacing of $20 \mu \mathrm{m}$. The deviation of the shape of the channels from the expected circular shape can be observed.

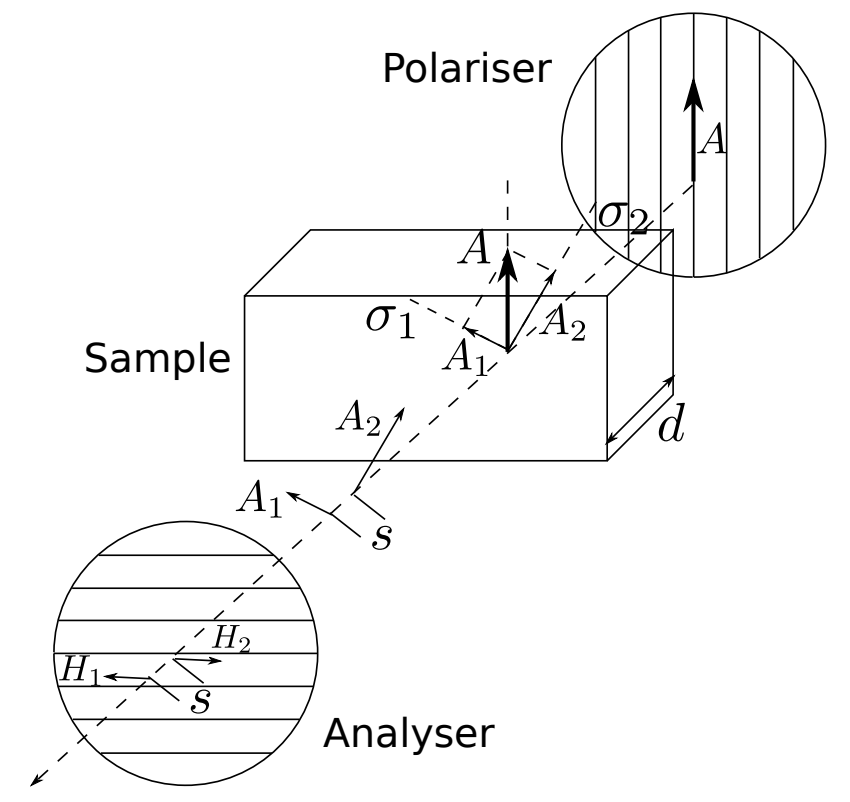

Figure 6.8.: Schematic of the setup measuring the induced stress in the diamond [102]. Polarised light is guided through the material. Depending on the induced stress, the speed of light varies for different directions of polarisation, leading to a shift in polarisation. This can be anlaysed by a second polariser. 


\section{6. $3 D$ Electrodes in optical grade $p C V D$ Diamond}

$\sigma_{1,2}$, which are perpendicular to each other. They are defined as

$$
\sigma=\frac{F}{A}
$$

with $F$ being the force perpendicular to the area $A$. The tension points in the direction of the force. If the absolute values of the two modules are not equal, the speed of light in the material along these moments differs. This results in a phase difference of the light with respect to the two modules of tension behind the material. The phase difference $\delta$ can be expressed as [102]

$$
\delta=C\left(\sigma_{1}-\sigma_{2}\right) \frac{s}{\lambda}
$$

with $\lambda$ being the wavelength of the light, $C$ a material specific constant, and $s$ the thickness of the sample. Consequently, if the tension inside the diamond is not uniform, a shift in polarisation can be observed.

This measurement was performed and analysed by H. Beck as part of his bachelors thesis, where a more in-depth discussion can be found [97]. In order to quantify the strength of the tension inside the diamond, the relative intensity $I$ of the polarisation shift is calculated via

$$
I=\frac{I_{\perp}}{I_{\perp}+I_{\|}}
$$

with $I_{\|}$and $I_{\perp}$ being the intensities for parallel and perpendicular polarisers, respectively. As the overall transmitted intensity for the channels is very low, a minimal total transmitted intensity $I_{\perp}+I_{\|}$is required. A visualisation of this calculation is shown in figure 6.9. What can be observed is that the diamond itself is not free of tension. Even areas without channels show a shift in polarisation. This is attributed to the polycrystalline structure of the sample. During production, the grains grow together and at grain boundaries tension arises. The result also shows that, in the immediate vicinity of the channels mechanical stress is induced. For the wider separation of $25 \mu \mathrm{m}$ and above, regions of increased stress remain isolated around the channels and does not connect with the stress from the neighbouring channel. For lower separations they do connect and for a separation of $10 \mu \mathrm{m}$ the channels themselves are that thick, that the inter-channel region can not be properly resolved.

Stress from a single channel alone is not critical, as no cracks resulting from these were observed. If the induced stress from several channels starts to connect over a large area, this might result in a crack of the diamond. In order to check, if this is problematic for realistic spacings, namely $50 \mu \mathrm{m}$ and $25 \mu \mathrm{m}$, two large areas of eight by 32 channels were grown with a laser power of $50 \mathrm{~mW}$ and a sample velocity of $50 \mu \mathrm{m} / \mathrm{s}$. Intentionally, these were produced in order to test the growth of channels, which do not extend fully through the diamond, but only reach one surface. Accordingly, the focal plane of the laser either started up to $30 \mu \mathrm{m}$ inside the diamond upstream of the seed side or stopped up to $30 \mu \mathrm{m}$ downstream of the exit side. As it turned out, almost all of these channels 


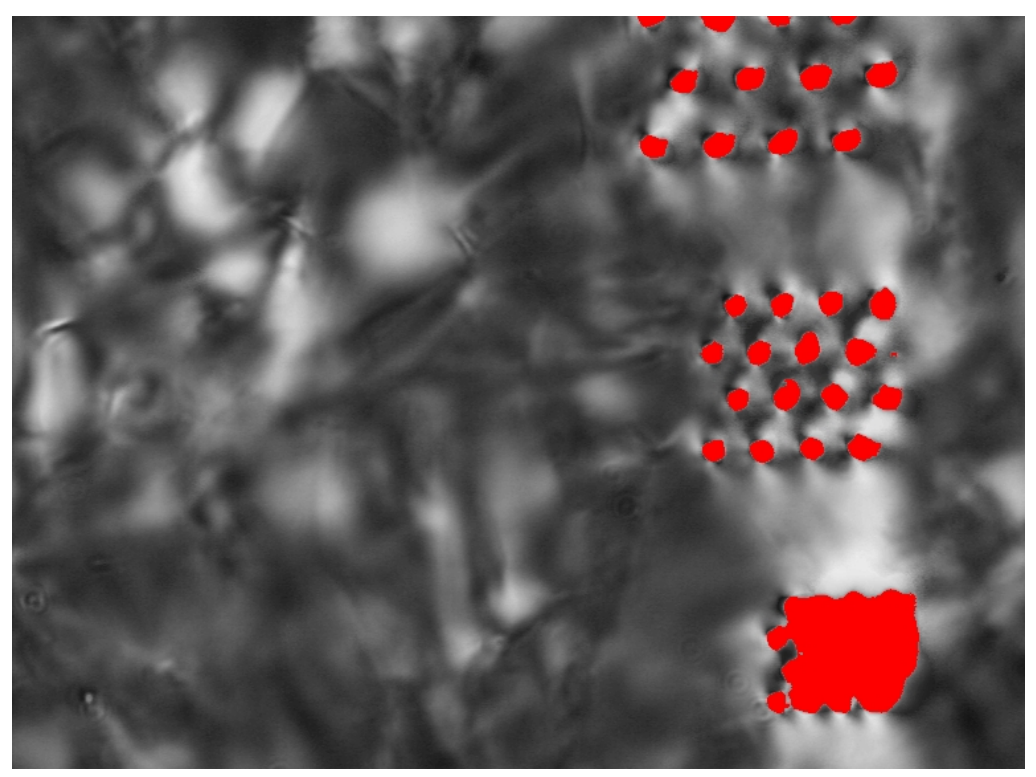

Figure 6.9.: Picture showing the relative intensity $I$ according to equation 6.3. Dark areas have a low, bright a high relative intensity. Areas with a low overall intensity are marked red and show the location of channels. The spacing of the channels is from top to bottom $20 \mu \mathrm{m}, 15 \mu \mathrm{m}$, and $10 \mu \mathrm{m}$ [97].

extended fully through the diamond, which could be due to the huge length of the focal plane of $31.8 \mu \mathrm{m}$. The visualisation of the induced mechanical stress for these two grids can be seen in figure 6.10 .

For the grid with a spacing of $50 \mu \mathrm{m}$ it is observed that regions of increased stress are exclusively located around the channels and do not connect to neighbouring channels. Consequently, such a spacing can be considered as safe. In case of the tighter spacing of $25 \mu \mathrm{m}$ in some rare cases a connection of the stress of two to five channels can be observed. Most of the time, the stress still does not connect. As a result, this spacing can also be considered as safe. This is especially important, as the spacing resembles the spacing in the short pixel direction of a possible pixel electrode geometry like the one shown in figure 5.9. As in this example the spacing along the long pixel direction is $125 \mu \mathrm{m}$, the situation in the diamond sample is more rigid than it would be in a real detector.

\subsection{D Writing in Diamond}

The very high measured resistance of the channels in Goe-pCVD-01, which is in the order of the resistance of diamond, could be due to surface effects. Such effects can prevent a proper contacting of the conductive channels and can have two causes. The first 


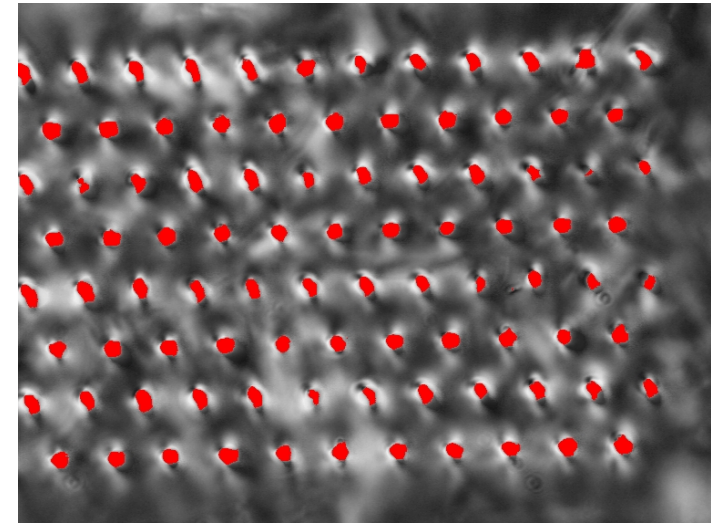

(a)

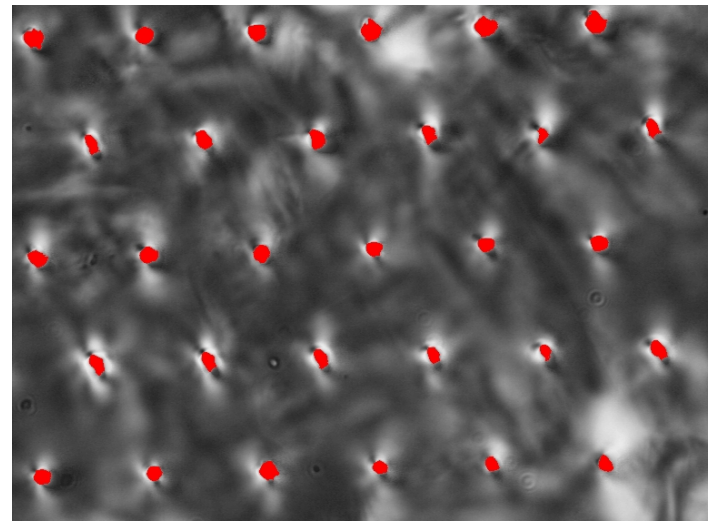

(b)

Figure 6.10.: Relative intensity for a large grid of channels with a spacing of (a) $25 \mu \mathrm{m}$ and (b) $50 \mu \mathrm{m}[97]$.

problem can be the improper cleaning of the diamond. This was already tried for the second batch of channels in Goe-pCVD-01 and did not yield a positive result. However, before metallising the third batch, the metal from the second batch was removed with acids, which might have also etched away some parts of the phase changed channels. The second cause is due to the different structure of the top of the channels for seed and exit side. On the exit side, the crater like structure can prevent a proper contact between the phase changed material and the metal.

Accordingly, the sample Goe-pCVD-03 is cleaned before the production of channels and special channels are grown, which have two contacts on the same side. This is challenging, as the phase change can only be performed in one direction of the beam, namely upstream. For example, starting on the seed side, moving the focal plane upstream, to the side and then downstream would not result in a $\mathrm{u}$-shaped channel. When moving the focal plane downstream, The phase changed material more upstream would absorb the beam, preventing new phase change downstream to occur. In order to grow channels with two contacts on one side, two individual channels are grown under a slight angle with respect to the surface. Both individual channels have a common point inside the diamond bulk, forming an intersection and effectively merging the two channels into one triangular channel.

For the production of such channels, the stage holding the diamond has to move not only parallel but also perpendicular to the beam. Accordingly, it was programmed to move in a way, that for the triangular channels the intersection is either $200 \mu \mathrm{m}$ or $300 \mu \mathrm{m}$ below the surface, from where the channel will be contacted. For this it has to be kept in mind that due to the diffractive index of 2.4 of diamond [103], a step of the stage of one micrometer length equals a move of the focal plane inside the diamond of $2.4 \mu \mathrm{m}$. 


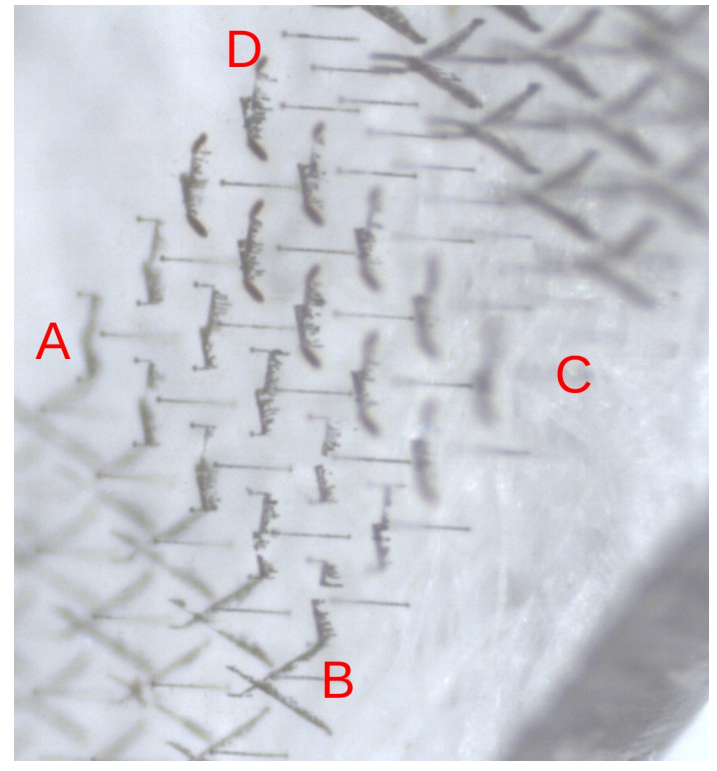

(a)

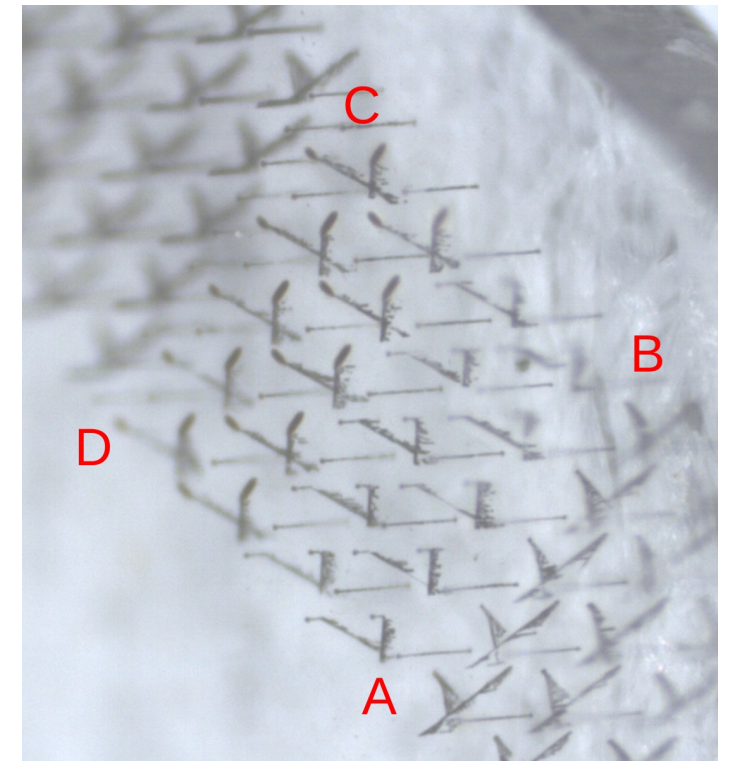

(b)

Figure 6.11.: (a) Tilted view from the seed side on triangular channels and (b) for the same channels with the sample rotated by $90^{\circ}$. The channels have two contacts on the seed side and an intersection inside the diamond bulk.

In order to ensure that the two individual channels forming the triangular channel do really connect, they are extended $50 \mu \mathrm{m}$ inside the diamond beyond the expected point of the intersection. A picture of some of these channels can be seen in figure 6.11. The two channels are perpendicular with respect to each other, when viewing from one of the surfaces of the sample. The reason for this geometry is the reuse of the mask from Goe-pCVD-01 for metallisation, which has a rectangular pattern. As shown in figure 6.5 the mask has on one side dots, which allows the individual measurement of the resistance of the triangular channels with contacts on this side. On the other side the mask consists of strip electrodes, triangular channels on this side cannot be measured individually, but only several in parallel. In total 40 triangular channels were produced. The varied parameters are the depth, where the intersection is located with $200 \mu \mathrm{m}$ or $300 \mu \mathrm{m}$ below the surface, the surface side, the channels will be contacted from, the velocity of the sample in beam direction with $50 \mu \mathrm{m} / \mathrm{s}$ and $100 \mu \mathrm{m} / \mathrm{s}$, and the power of the laser with $50 \mathrm{~mW}$ and $100 \mathrm{~mW}$. For each set of parameters five channels were produced.

The resulting channels show, that the production of channels which are not parallel to the beam is possible. However, most of the channels show significant fraying. This is especially true for channels grown with a laser power of $100 \mathrm{~mW}$. Here, the frays are longer than for a laser power of $50 \mathrm{~mW}$ and, like the main channel itself, thicker. The frays are all located downstream of the main channel. This indicates that the phase change of 
6. $3 D$ Electrodes in optical grade $p C V D$ Diamond

\begin{tabular}{rrrr}
\hline Power $[\mathrm{mW}]$ & Velocity $[\mu \mathrm{m} / \mathrm{s}]$ & Measured distance $[\mu \mathrm{m}]$ & Focal distance $[\mu \mathrm{m}]$ \\
\hline 50 & 50 & $305 \pm 4$ & 350 \\
50 & 100 & $305 \pm 5$ & 350 \\
50 & 50 & $210 \pm 7$ & 250 \\
50 & 100 & $215 \pm 8$ & 250 \\
100 & 50 & $269 \pm 9$ & 350 \\
100 & 100 & $275 \pm 10$ & 350 \\
100 & 50 & $170 \pm 9$ & 250 \\
100 & 100 & $169 \pm 9$ & 250 \\
\hline
\end{tabular}

Table 6.3.: Mean values of the position of the first phase change in the diamond bulk relative to the surface of the exit side for channels with contacts on the exit side. The position of the focal plane relative to this surface is also given.

\begin{tabular}{rrrr}
\hline Power $[\mathrm{mW}]$ & Velocity $[\mu \mathrm{m} / \mathrm{s}]$ & Measured distance $[\mu \mathrm{m}]$ & Focal distance $[\mu \mathrm{m}]$ \\
\hline 50 & 50 & $175 \pm 5$ & 250 \\
50 & 100 & $171 \pm 7$ & 250 \\
100 & 50 & $142 \pm 8$ & 250 \\
100 & 100 & $136 \pm 6$ & 250 \\
\hline
\end{tabular}

Table 6.4.: Mean values of the position of the final phase change in the diamond bulk relative to the surface of the exit side for channels with contacts on the seed side. The position of the focal plane relative to this surface is also given.

the main channel happens at the minimal fluence needed to induce a phase change. In case of the tilted channels, the focal area does not only move along the beam axis, but also sideways. Accordingly, the already produced channel can not completely absorb the laser beam. This allows spontaneous phase change to occur in the high fluence areas farther downstream. These then grow until the fluence drops below the threshold of continued phase change. This is exactly where the main channel is located. The length of individual frays depends on their origin. For channels with contacts at the exit side, long frays start at the surface and can reach a length of about $200 \mu \mathrm{m}$. Frays, which begin inside of the diamond bulk achieve only a maximum length in the order of $100 \mu \mathrm{m}$. This shows, that the threshold for phase transformation is lower on the surface and also at interfaces between phase changed material and diamond, than in pure diamond.

The depths of the end of the channels inside the diamond with respect to the exit side are summarised in table 6.3 for channels with contacts on the exit side and in table 6.4 for channels with contacts on the seed side. These were measured using microscope photos of the tilted diamond. The resolution of one pixel is about $6 \mu \mathrm{m}$. For all channels the phase change stops upstream of the position of the focal plane. This is expected, as 


\begin{tabular}{rrr}
\hline Contact side & Power $[\mathrm{mW}]$ & Relative distance $[\mu \mathrm{m}]$ \\
\hline exit & 50 & $43 \pm 3$ \\
exit & 100 & $79 \pm 5$ \\
seed & 50 & $76 \pm 4$ \\
seed & 100 & $112 \pm 5$ \\
\hline
\end{tabular}

Table 6.5.: Difference between the measured distance to the exit side and the position of the focal plane. For all settings, the phase change happens upstream of the focal plane.

the phase change happens upstream of the focal plane. Within the uncertainties, channels grown with different velocities but with the same power stop at the same position. Therefore, the velocity has, for the chosen values, no or only a minor impact on the location of the phase change relative to the focal plane. This might be different for lower velocities due to the formation of micro defects.

As expected, the parameter with the largest influence on the relative position of the phase change is the power of the laser. In table 6.5 the resulting differences between the position of the focal plane and the distance of the channel to the exit side are summarised. For channels, which start in the diamond bulk, the relative distance between location of the focal plane and the actual phase change is $(43 \pm 3) \mu \mathrm{m}$ and $(79 \pm 5) \mu \mathrm{m}$ for a laser power of $50 \mathrm{~mW}$ and $100 \mathrm{~mW}$, respectively. This yields an increase in the relative distance of $36 \mu \mathrm{m}$ with the increase of laser power. The same increase if the channels end inside the diamond bulk. This increase of relative distance is expected, as for a higher laser power, the fluence more upstream of the focal plane is sufficiently high to initiate phase change.

Furthermore, the relative distance between location of the focal plane and the actual phase change depends on whether the channel starts or ends in the diamond bulk. Channels with contacts on the exit side start their phase change inside the diamond bulk, which requires spontanous phase change. As this has a higher fluence threshold than phase change on the surface, they should start more closely to the focal plane. This is observed and the shift of relative distance for the same laser power is also consistent.

Another observation is that all channels which should only be contacted from the seed side and have their intersection $300 \mu \mathrm{m}$ above the seed side, do reach the exit side. A reason for this could be the low distance to the exit side at the planned end of the channel. As the channels extend $50 \mu \mathrm{m}$ further into the diamond after the intersection, this leaves only a distance to the exit side of about $150 \mu \mathrm{m}$. This distance is further reduced, as the phase change happens upstream of the focal region, as shown above. However, the measured relative distances from above are too small to explain, why these channels reach the exit side. Another reason could be the movement of the stages for this setting. 


\section{6. $3 D$ Electrodes in optical grade $p C V D$ Diamond}

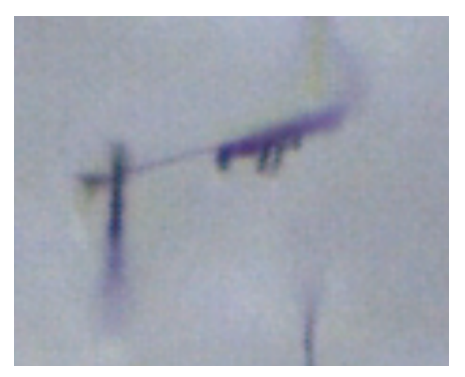

Figure 6.12.: Picture of a tilted channel with parts of it having a significantly smaller diameter than the normally observed thickness.

The shutter of the beam is only shut, when all axes of the stage are at their designated destinations. A mismatch of the velocities of the stages could yield a longer time of exposure at the end of the channel than intended.

In some rare cases significantly thinner parts of channels can be observed. An example can be seen in figure 6.12. Such channels are only observed for a laser power of $50 \mathrm{~mW}$. The small diameter can be explained by the hypothesis that the phase change happens at the very upstream limit, where the fluence barely exceeds the threshold for continued phase change. These thin parts continue with parts which have a normal thickness. These are always initiated by a fray, which allows the phase change to happen more downstream, resulting in the observed normal thickness.

The triangular channels occupy only three contacts ${ }^{2}$ in a two by two grid. Consequently, the remaining contact is used to further investigate the influence of the velocity of the sample on the growth of the channels. The parameters of these straight channels are velocities of $50,100,200,400,800 \mu \mathrm{m} / \mathrm{s}$ and powers of $50 \mathrm{~mW}$ and $100 \mathrm{~mW}$. For each combination of these parameters, ten channels were produced. All of these fully extend through the diamond.

A first measurement of the resistance of the triangular and straight channels yielded no resistance lower than the resistance of the diamond bulk. Unfortunately, the diamond was lost during handling, which prevented a more detailed investigation. Nevertheless, the results hint that the very low conductivity of the channels is probably not only due to surface effects. Otherwise the channels with contacts from the seed side only, where no crater like structure is observed, should have yielded a lower resistance than the resistance of diamond. This indicates, that the phase transformation might be insufficient and still a large proportion of the carbon atoms is $s p^{3}$ bound.

\footnotetext{
${ }^{2}$ The channels reach the surface under two contacts and have their intersection under the third one.
} 


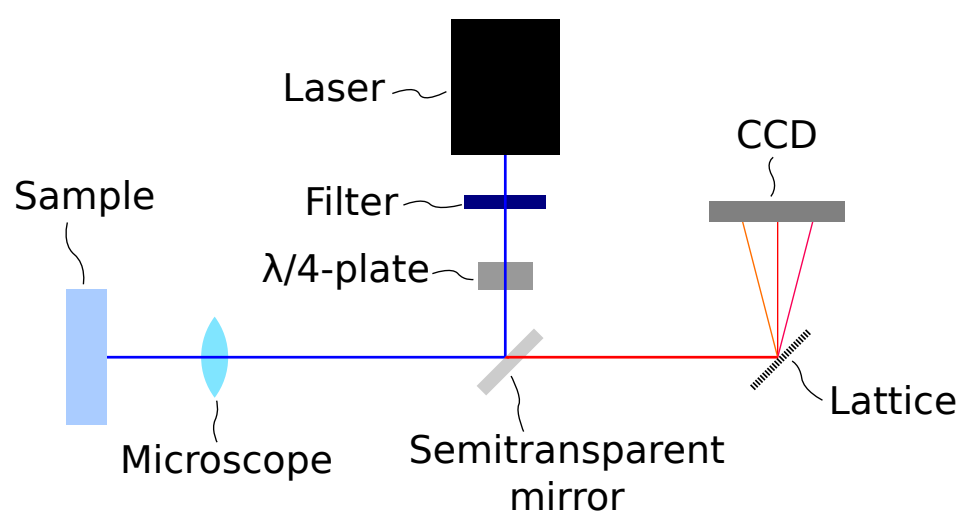

Figure 6.13.: Drawing of a Raman spectroscopy setup.

\subsection{Raman Spectroscopy}

\subsubsection{Raman basics and setup}

The measurement of the resistance does not yield any information about the actual composition of the phase changed material. Furthermore, in order to measure it, the sample has to be metallised. This involves manipulations of the surface, which could affect the outcome of the measurement.

A technique, which allows the investigation of bound states is Raman spectroscopy, which was discovered in 1928 by C.V. Raman [104]. A schematic setup is shown in figure 6.13. The sample is illuminated with a laser and the spectrum of the backscattered light is analysed. Most photons scatter elastically with the material and thus their wavelength is not changed. In some cases the photons scatter inelastically and absorb or emit a phonon, altering the wavelengths of the photons. A phonon is a quantum mechanical representation of a vibrational mode, that behaves like a particle [105]. It has properties of a real particle like e.g. energy, mass and frequency, but cannot exist outside of the crystal. The vibrational modes a crystal can have are discrete and depend on the lattice structure of the crystal. Consequently, the energies of the phonons in a crystal are characteristic for it. These energies can be determined by measuring the shift of wavelength of the scattered photons. As a result, with Raman spectroscopy it is possible to measure the composition of the phase changed material.

A schematic drawing of the dominant scattering processes of photons in a crystal is given in figure 6.14. In case of elastic scattering, the direction of the photon may be changed, but not its energy. The dominant process for this is Rayleigh scattering. If the photon scatters inelastically and emits a phonon, its wavelength is shifted to higher values. This process is called Stokes-Raman scattering. The absorption of a phonon 
6. 3D Electrodes in optical grade $p C V D$ Diamond

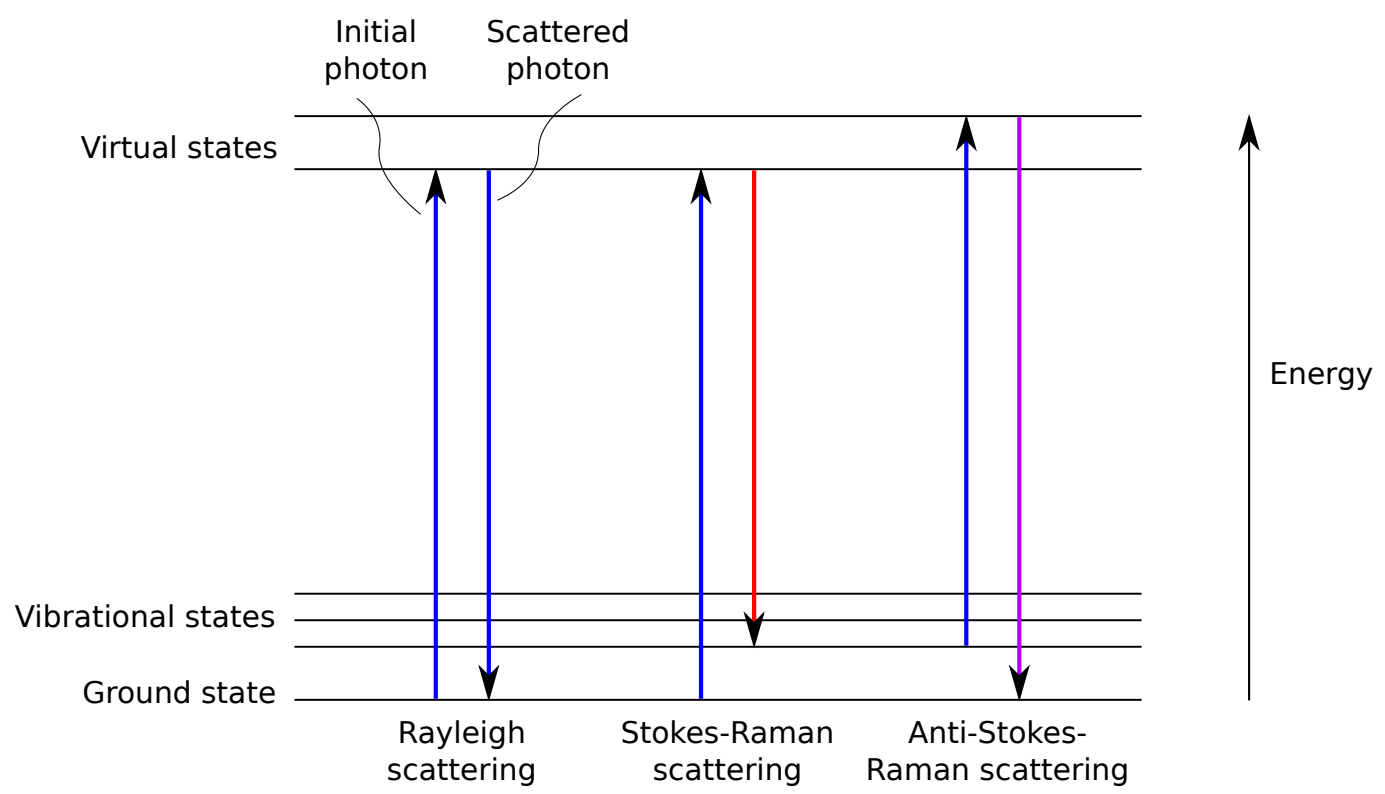

Figure 6.14.: Schematic drawing of possible scattering processes of photons at a crystal lattice. The photon can scatter elastically or inelastically. In the later case either a phonon is emitted or absorbed. The possible energies of such phonons are represented in the schematic view by the vibrational states. 
is also possible. In this so called Anti-Stokes-Raman scattering the wavelength of the photon is lowered. The premise for this process is the existence of such a phonon. As more phonons are present at higher temperatures of the sample, the cross section of Anti-Stokes-Raman scattering increases with the temperature. However, the cross section is significantly lower than that of Stokes-Raman scattering for samples at room temperature [105].

The Raman shift is typically quoted in $\mathrm{cm}^{-1}$. This can be easily converted into the energy of the emitted or absorbed phonon, as

$$
\Delta E=\frac{h c}{\Delta \lambda} .
$$

In order to analyse the bonds, an intensity profile of the shifted light is recorded.

For this thesis, the Raman setup in the mineralogy department of the faculty for geology was used. The wavelength of the laser of a Raman setup is chosen such that it does only excite the electrons, but does not ionise them. In this case the wavelength is $488 \mathrm{~nm}$. It is critical to ensure that the laser is monochromatic in order to exactly know the excitation energy. For this reason the laser is guided through several filters, which are transparent only for its main wavelength. Due to the crystal structure, the scattering on the sample depends on anisotropic effects. Consequently, the polarisation of the laser is changed from linear to circular via a $\lambda / 4$-plate, as circular polarised light is insensitive to such effects. The laser light is further guided onto a semitransparent mirror, which only reflects light with the wavelength of the laser. It is reflected into a microscope and focused on the sample. The backscattered light follows the same route back through the microscope and onto the semitransparent mirror. Here, only the shifted wavelengths are going through. They then propagate onto a lattice. The resulting wavelength separated beam is detected with a photo detector, which counts the number of photons. This detector is located with respect to the lattice in a way, that it detects the photons, which are Stokes-Raman shifted. One row of pixels of the sensor equals about $2 \mathrm{~cm}^{-1}$. Prior to the measurement, the setup is calibrated with a pure silicon sample.

\subsubsection{Calibration and Verification of the Raman Setup}

In order to be able to interpret the Raman spectra for the channels, first the spectra for pure diamond and graphite have to be measured. Diamond has only one phonon mode, which is in first order Raman active [106]. First order means that only one phonon is emitted or absorbed. This results in one sharp peak at approximately $1332 \mathrm{~cm}^{-1}$. For the sample Goe-pCVD-02 such a signal is shown in figure 6.15. The laser of the Raman spectrometer was focused on the surface of the sample at least $500 \mu \mathrm{m}$ away from channels or edges. A clean peak at $(1334 \pm 2) \mathrm{cm}^{-1}$ can be seen without any other visible reflex in the range of $1000 \mathrm{~cm}^{-1}$ to $2000 \mathrm{~cm}^{-1}$. 


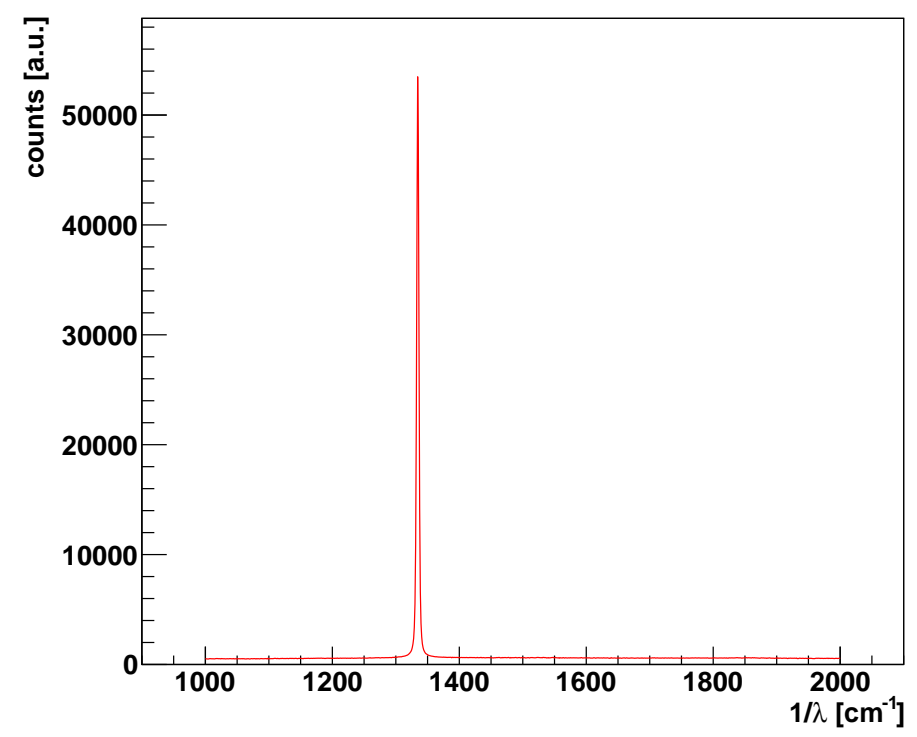

Figure 6.15.: Raman spectrum of pure diamond. The diamond peak is clearly visible.

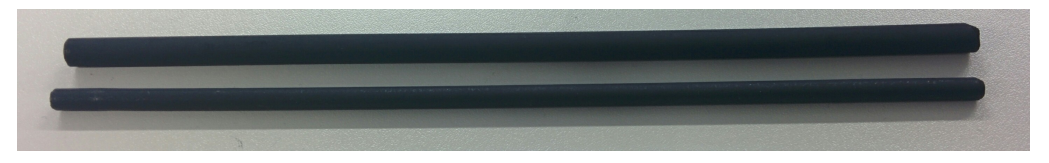

Figure 6.16.: Graphite sticks used for reference Raman spectra of disordered, conductive graphite.

For a perfect graphite crystal, only one Raman mode at $1581 \mathrm{~cm}^{-1}$ is expected [107]. However, if graphite is produced in the channels, it is expected to be disordered to some extend. In such a case, two broad peaks are measurable with Raman spectroscopy [108]. One is the broadened peak of perfect graphite, the so called g-peak. It is located around $1580 \mathrm{~cm}^{-1}$. The second peak arises from the disordering of the crystal and is called d-peak. Accordingly, the intensity of this peak depends on the grain size. The peak is centred around $1350 \mathrm{~cm}^{-1}$. These two peaks have been measured using two conductive graphite sticks. A photo of these is shown in figure 6.16. Both sticks are $(20.0 \pm 0.5) \mathrm{cm}$ long. The thicker one has a diameter of $(6.4 \pm 0.1) \mathrm{mm}$ and a resistance of $(1.9 \pm 0.1) \Omega$. The thinner stick has a diameter of $(4.9 \pm 0.1) \mathrm{mm}$ and a resistance of $(2.3 \pm 0.2) \Omega$. This yields a resistivity of $(0.31 \pm 0.02) \Omega \mathrm{mm}$ and $(0.22 \pm 0.02) \Omega \mathrm{mm}$ for the thick and thin graphite stick, respectively. Their Raman spectra can be seen in figure 6.17(a) and figure 6.17(b) for the thicker and thinner stick, respectively. For both sticks the g-peak and the d-peak are observed. As expected, the g-peak is located around $1580 \mathrm{~cm}^{-1}$ and the d-peak around $1350 \mathrm{~cm}^{-1}$. This shows that even for very conductive graphite, a disordering is present in the crystal. 


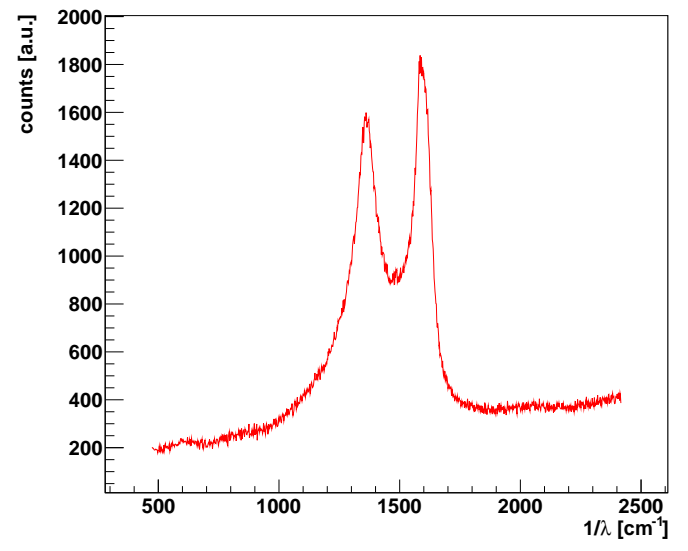

(a)

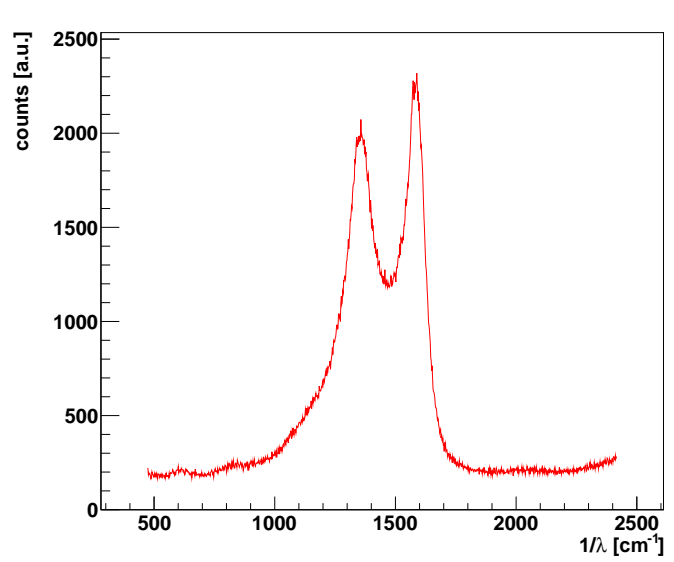

(b)

Figure 6.17.: Raman spectra of (a) the thick and (b) the thin graphite stick shown in figure 6.16. The g- and d-peak are clearly visible.

This disordering can be quantified by the relative intensities of the two peaks. Starting from a mean ordered crystal size of $2 \mathrm{~nm}$ the relative intensity of the d-peak decreases inversely with growing ordered crystal size [109]. For crystal sizes below $2 \mathrm{~nm}$, the material is called amorphous carbon. In this region the relative intensity of the d-peak with respect to the g-peak increases quadratically with growing crystal sizes. This is attributed to the increasing presence of sixfold carbon rings, which cause the d-peak.

Between pure diamond and pure graphite other materials consisting solely of carbon can be identified. A schema of this is shown in figure 6.18. If around $10 \%$ to $40 \%$ of the atoms are $s p^{3}$ bound and the remaining atoms are $s p^{2}$ bound, the material is called amorphous carbon. For a share of up to $90 \% s p^{3}$ bound atoms, the material is identified as diamond like carbon (DLC). The channels produced inside the diamond are expected to consist of a mixture of these materials. The exact mixture depends on the growth parameters, namely the power of the laser and the velocity of the sample.

The Raman spectrum of DLC consists of two broad peaks. One is located at $1540 \mathrm{~cm}^{-1}$ and the other one between $1300 \mathrm{~cm}^{-1}$ and $1350 \mathrm{~cm}^{-1}$ [110]. With decreasing $s p^{3}$ contribution, the relative intensities and the peak positions change, resembling more the g- and d-peaks of amorphous graphite. Accordingly, the mixture of $s p^{2}$ and $s p^{3}$ bound atoms in the channel can be determined by these values. A detailed discussion of this can be found in a publication by Ferrari and Robertson [109].

Before measuring the channels, the diameter of the focal plane of the Raman laser has to be determined, using a silicon diode. It has partially a aluminium electrode on top, which has a sharp edge to the silicon. The edge was produced with photolitography, 


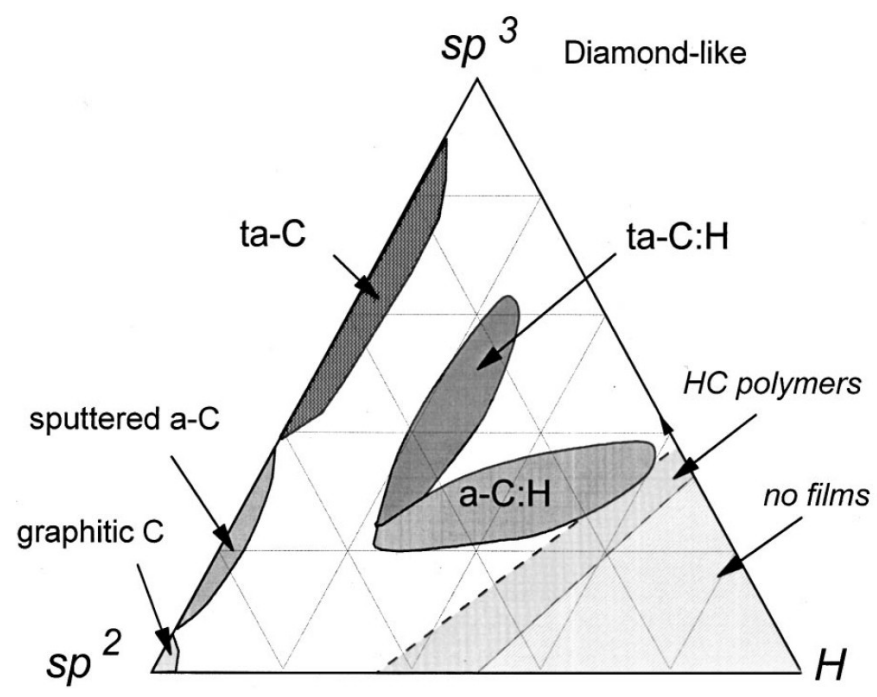

Figure 6.18.: Phase diagram of $s p^{2}, s p^{3}$, and hydrogen bound carbon [109]. For this thesis relevant are the phases for different ratios of $s p^{2}$ and $s p^{3}$ bound carbon. With decreasing contribution of $s p^{2}$ bound carbon, these are graphite, amorphous carbon (a-C), diamond like carbon (DLC, ta-C), and diamond.

resulting in a deviation from the perfect edge of less than $0.25 \mu \mathrm{m}$. The focus of the Raman laser is moved across this interface like shown in figure 6.19. Focused on the silicon, the Raman spectrum shows the silicon peak at $520 \mathrm{~cm}^{-1}$. Aluminium has no Raman mode in the recorded wave number interval and absorbs the laser completely. Accordingly, moving the focus over the edge of the metal electrode, the silicon peak disappears. This has been measured at four different spots. In two cases the edge is crossed moving the focus vertically and horizontally, respectively. The step size is $0.1 \mu \mathrm{m}$ with a total of 100 steps. An example of the resulting spectra is shown in figure 6.20(a).

In order to calculate the diameter of the focus, the disappearance of the silicon peak has to be quantified. For this, the background of the spectra is determined by fitting a linear function with the range of $1100 \mathrm{~cm}^{-1}$ to $2400 \mathrm{~cm}^{-1}$ to each spectrum. The intensities are corrected by this value and the entries from $500 \mathrm{~cm}^{-1}$ to $540 \mathrm{~cm}^{-1}$ are summed up. The result is shown in figure 6.20(b). Assuming a Gaussian beam shape, the diameter of the focus can be determined by fitting the error function

$$
I(x)=c \cdot \operatorname{Erfc}\left(\frac{x-p}{\sqrt{2} \sigma}\right)+d
$$

to the data. Here, $c$ and $d$ are scaling factors and $p$ is the position of the edge. $2 \sigma$ denotes the diameter of the focus in which $39.3 \%$ of the intensity is deposited [111]. The results for the four different spots are given in figure 6.21(a). It can be seen that the variance between spots is larger than the statistical error suggests. One contribution to this 
6.6. Raman Spectroscopy

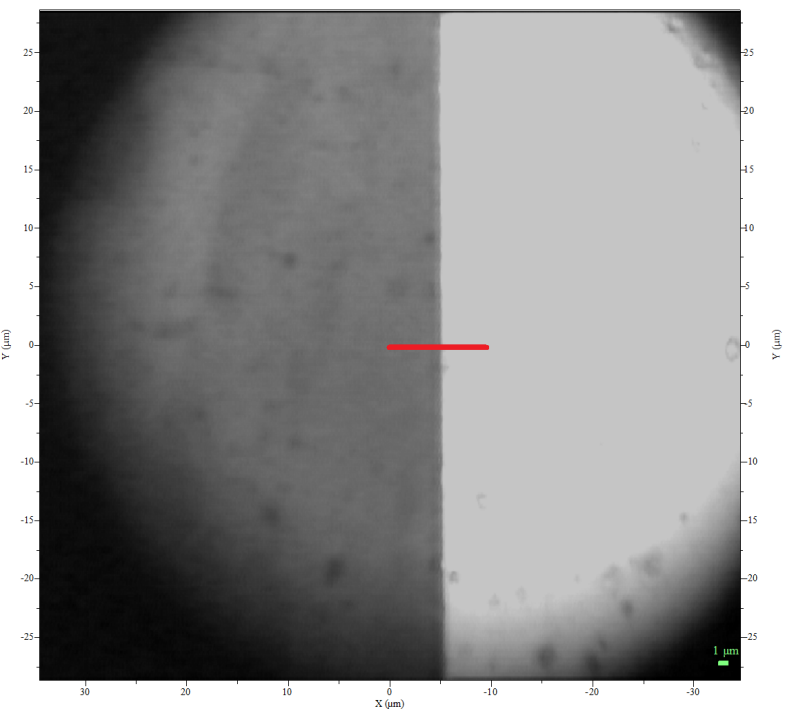

Figure 6.19.: Photo of the surface of the silicon diode used to determine the spot size of the focus of the Raman laser. The silicon appears dark and the aluminium electrode bright. The movement of the focal plane on the surface is indicated by the red line.

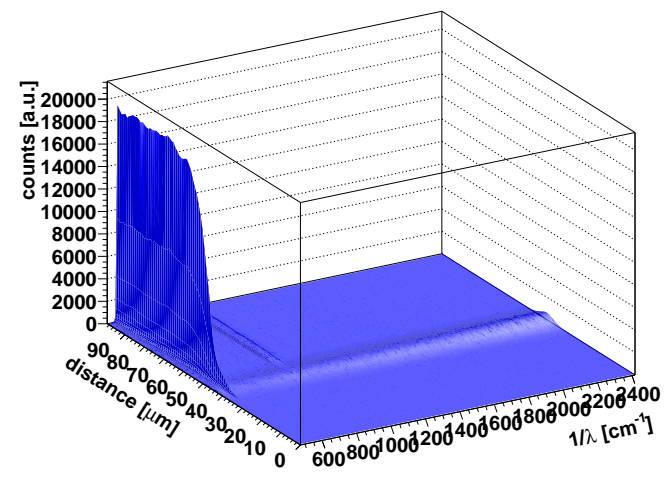

(a)

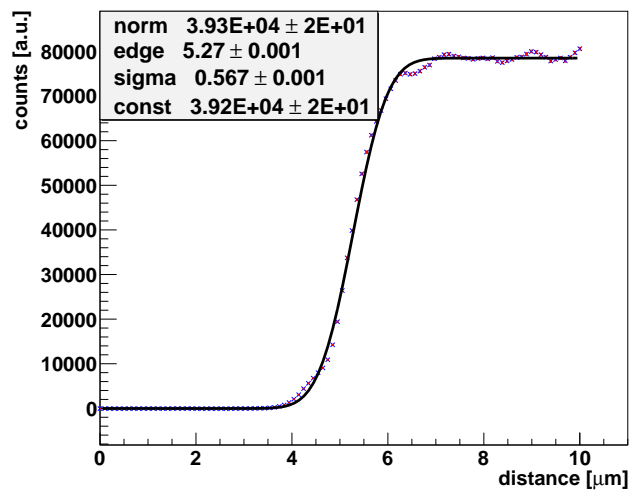

(b)

Figure 6.20.: Spectra of the diode while moving the Raman laser over the edge of an aluminium electrode (a). The disappearance of the silicon peak can be observed. From the integral of this peak, the diameter of the focus can be calculated (b). 
6. $3 D$ Electrodes in optical grade $p C V D$ Diamond

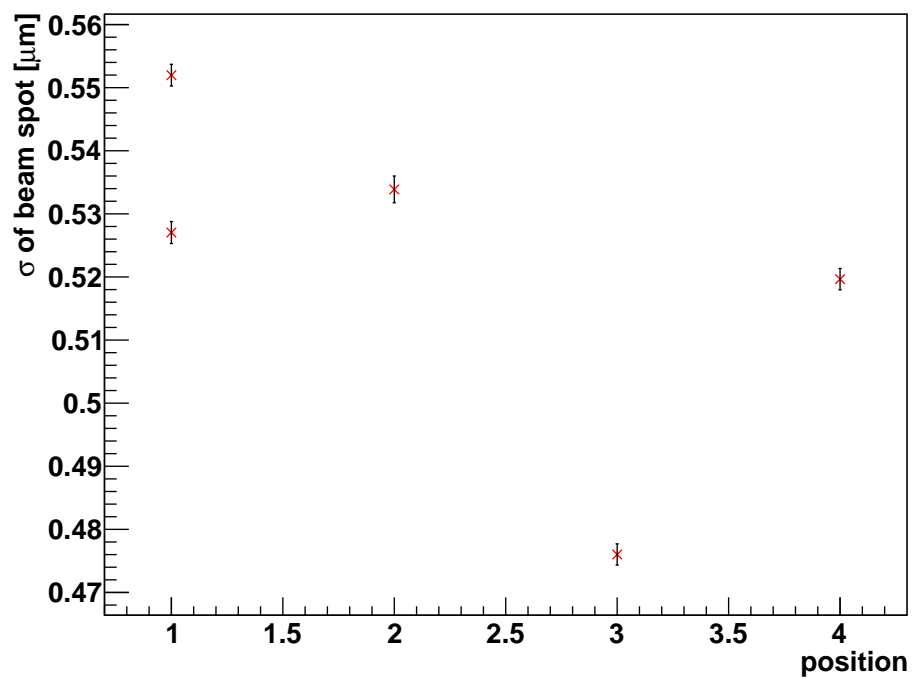

(a)

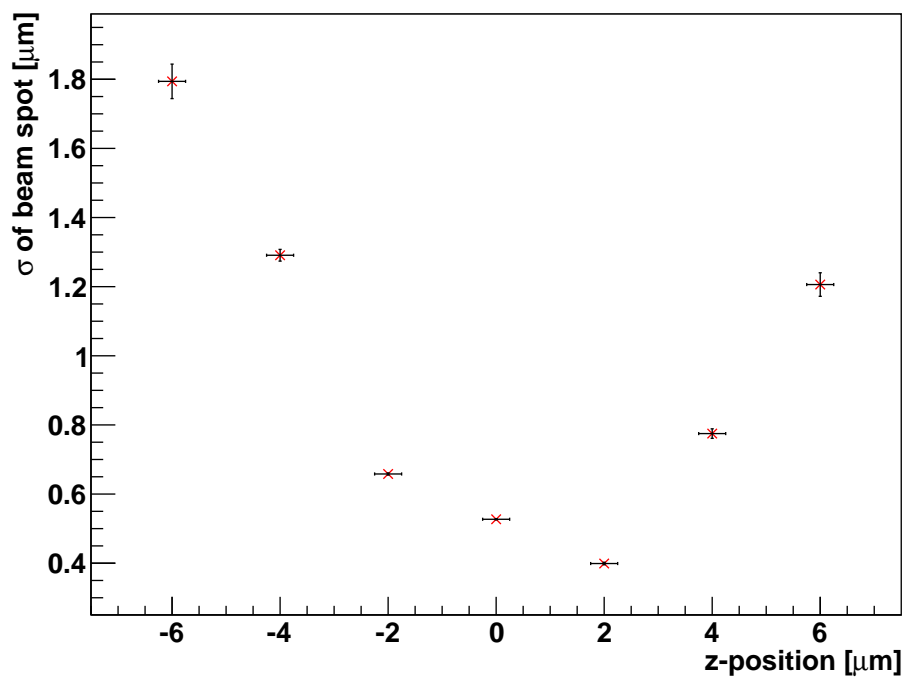

(b)

Figure 6.21.: Size of the focus measured at four different spots (a) and its dependence on the z-position of the sample (b). This position is measured with respect to the focus of the camera of the Raman setup. For positive values, this camera focus is above, for negative values below the surface of the diode. 
systematic error is the angle of the edge with respect to the movement of the focus. This has to be $90^{\circ}$ in order to measure the diameter of the focus. As the diode was positioned manually, an uncertainty of few degrees is possible. For one spot, the measurement was repeated with the trajectory shifted by a few micrometer along the edge. The deviation between the measurements shows that the angle between the surface of the silicon diode and the metallization is not exactly 90 degrees. Therefore, the measurement yields only an upper limit of $(1.04 \pm 0.01) \mu \mathrm{m}$ on the diameter of the laser beam spot. This is sufficiently small, as the smallest diameter of a channel is $4 \mu \mathrm{m}$. In this case $86.5 \%$ of the laser intensity would be on the phase changed material [111]. The size of the focus also depends on the z-position of the sample. As shown in figure 6.21(b), the minimal size of the laser beam spot is not achieved if the microscope is focused onto the surface. Instead, it is located about $1 \mu \mathrm{m}$ downstream. This is advantageous for the measurement of the crater like structures, as the microscope is focused on the level of the surrounding surface.

\subsubsection{Raman Spectra of Goe-pCVD-02}

The diamond sample Goe-pCVD-02 is used for Raman spectroscopy, as its surface has not been exposed to any chemicals before, nor was it metallised. The former could remove material from the channel and the latter prevents inspection via Raman spectroscopy. The resulting spectra for two channels grown at a laser power of $50 \mathrm{~mW}$ and a sample velocity of $50 \mu \mathrm{m} / \mathrm{s}$ for both the seed and the exit side is shown in figure 6.22. In all spectra the diamond peak is clearly visible.

Both spectra from the seed side show nearly the same qualitative characteristics. In both of them the d- and g-peak of $s p^{2}$ bound carbon is visible. However, the diamond peak is very dominant. It has also be taken into account, that the signals originating from $s p^{2}$ bound atoms are up to 230 times more sensitive to visible Raman spectroscopy than from $s p^{3}$ bound atoms [112]. As the seed side is after the phase change not exposed to the femtosecond laser any more and the pressure from the surrounding material is lower than inside the diamond bulk, the highest ratio of $s p^{2}$ bound atoms can be found here. Nevertheless, the Raman spectra show a very low share of phase changed material. As the growth parameters are typical parameters used also for the other diamonds, this explains why a very high resistance has been measured for such channels.

On the exit side a lower contribution from $s p^{2}$ bound carbon is expected, as some of the material is evaporated. This can be seen in the spectra in figure $6.22(\mathrm{~b})$ where in case of a spacing of $25 \mu \mathrm{m}$ the g-peak is nearly invisible. Considering the enhanced visibility of $s p^{2}$ sites, it can be concluded, that almost all of this material is removed on the exit side. This result agrees with the high resistance of metallised channels. In both spectra a broad signal at $650 \mathrm{~cm}^{-1}$ can be seen, which originates from a contamination on the surface. 
6. 3D Electrodes in optical grade pCVD Diamond

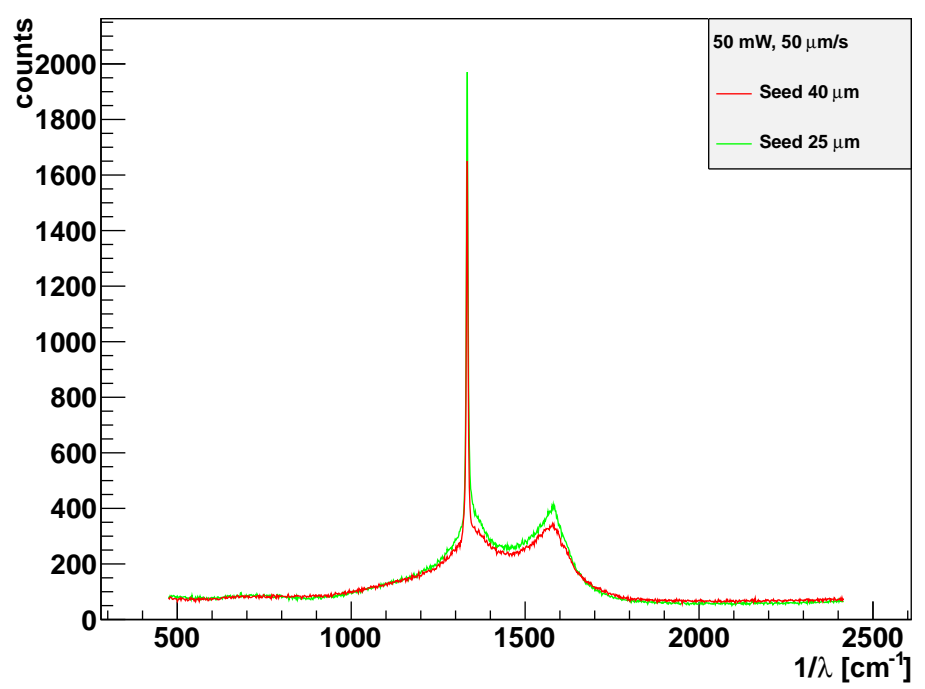

(a)

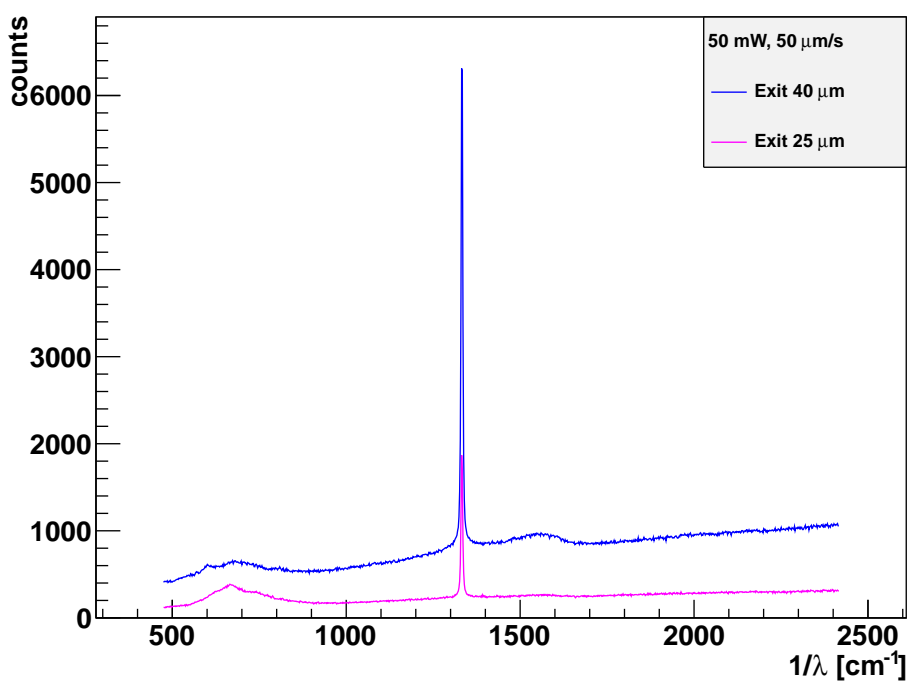

(b)

Figure 6.22.: Raman spectra of two channels on Goe-pCVD-02 for (a) the seed and (b) the exit side. The channels were produced with a laser power of $50 \mathrm{~mW}$ and a sample velocity of $50 \mu \mathrm{m} / \mathrm{s}$. The distance to their neighbours is either $25 \mu \mathrm{m}$ or $40 \mu \mathrm{m}$. 
CHAPTER 7

\section{D Electrodes in detector grade polycrystalline Diamond}

This chapter describes the successful production of conductive channels in a detector grade polycrystalline diamond sample, which is introduced in section 7.1. The production of the conductive channels and changes to the femtosecond laser setup are summarised in section 7.2. These channels are investigated thoroughly and in section 7.3 their Raman spectra are discussed, which yield indications of the structure of the phase changed material. The crater like structure on the exit side is measured with an atomic force microscope and the results are presented in section 7.4. In section 7.5 the measured resistances of the conductive channels are discussed.

\subsection{Detector grade polycrystalline Diamond Sample}

The diamond sample used for all measurements presented in this chapter is a detector grade polycrystalline diamond. Detector grade means, that the diamond has a higher CCD than lower grade samples described in chapter 6 . This sample was lent to us by the University of Manchester, which produced conductive channels in similar samples before. Accordingly, this eliminates the uncertainty, due to the use of low grade diamond material. The sample is about $1 \times 1 \mathrm{~cm}^{2}$ large and $500 \mu \mathrm{m}$ thick.

\subsection{Produced 3D Electrodes}

As seen in section 6.6 , the very high resistivity of the channels can have two sources. The first one is the overall insufficient phase transition from $s p^{3}$ bonds to $s p^{2}$ bonds. This results in a very high resistivity of the channel, which is unacceptable for electrodes. Second, the ratio of $s p^{2}$ to $s p^{3}$ is lower on the exit side of the channel, as parts of the conductive material are evaporated, forming a crater like structure. 


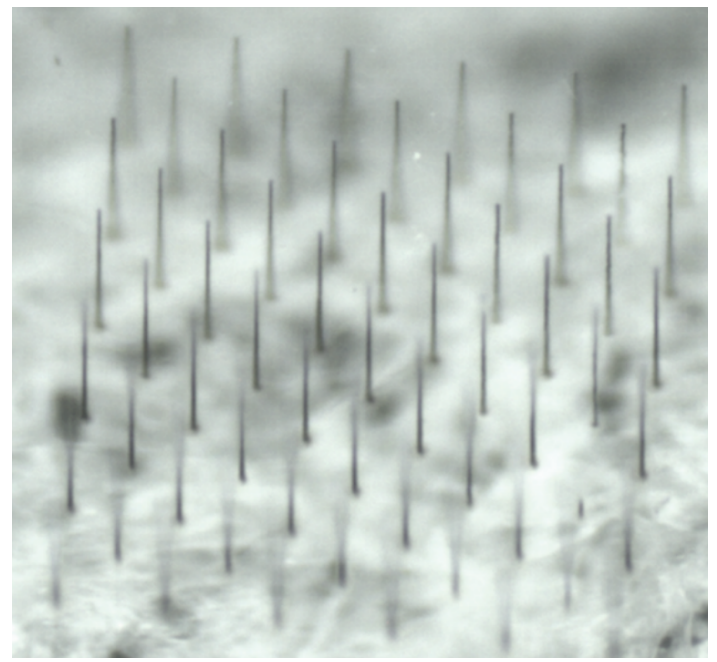

(a)

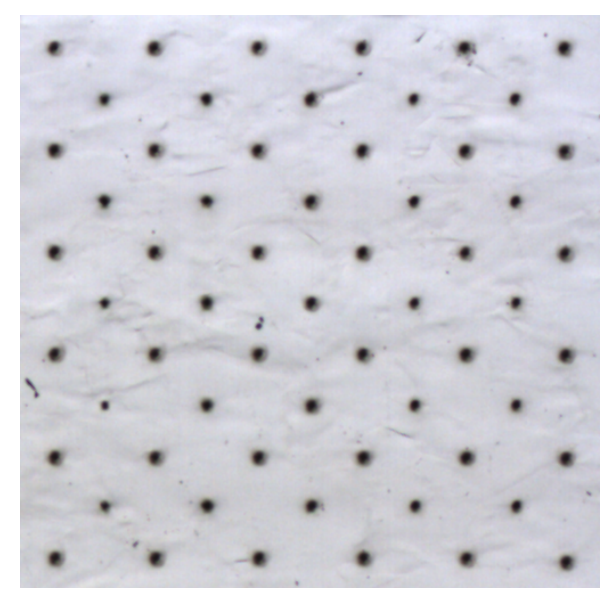

(b)

Figure 7.1.: Photos of the four grids produced in the first batch. In (a) the diamond is tilted with the seed side on top. In (b) the exit side is shown. The structure of a six by six channels grid interleaved with a grid of five by five channels is visible.

Both sources have been addressed by changes in the femtosecond laser setup. In order to achieve a higher fluence, the focal lens is replaced and the size of the focal plane is now $3.9 \mu \mathrm{m}$ in length and $1.6 \mu \mathrm{m}$ in diameter [96]. This yields for the same laser power a 8.2 times higher fluence. With this higher fluence, more energy is locally available for a more complete phase transformation. The tighter focus also results in a higher aperture. This means, that the fluence decreases much faster than for the previously used focal lens, as the beam before and after the focal plane opens at a larger angle. A phase change closer to the actual focal plane is the result. This would ease the production of channels not fully expanding through the sensor.

In order to reduce the evaporation of phase changed material on the exit side, a faster stage is used to move the diamond sample along the beam direction. This stage allows velocities of up to $10 \mathrm{~mm} / \mathrm{s}$. Combined with the higher aperture of the focal lens, the number of high fluence pulses on the exit side is significantly reduced.

Two batches of channels were produced. The first batch consists of four grids of six by six channels. A picture of one of these grids is shown in figure 7.1. All channels within one grid have the same growth parameter, namely a laser power of $25 \mathrm{~mW}$ or $60 \mathrm{~mW}$ and a velocity of $200 \mu \mathrm{m} / \mathrm{s}$ or $2 \mathrm{~mm} / \mathrm{s}$. The distance between the channels is $120 \mu \mathrm{m}$. These large grids are each interleaved with a grid of five by five channels. The channels of the smaller grid have as well a spacing of $120 \mu \mathrm{m}$ and are each located in the centre of a two by two channel cell of the large grid. This results in a distance of $85 \mu \mathrm{m}$ to 
their nearest neighbour for all channels. The small grids are grown with laser powers of $10,25,60,150 \mathrm{~mW}$ and velocities of $50,200,2000,10000 \mu \mathrm{m} / \mathrm{s}$. For each parameter combination at least five channels are produced. The reason for this structure is that the intended mask for metallisation allows individual contacting of channels in the small grids only.

The values for the laser power include one setting, which is comparable to the power settings used before. A laser power of $10 \mathrm{~mW}$ with the new focal lens yields the same fluence, achieved by the previous setup with a power of $82 \mathrm{~mW}$. From the Raman spectra of Goe-pCVD-02 it is suspected that this fluence is too low to sufficiently change the phase of the carbon. However, this setting is included in order to better understand, why previously produced channels have a very high resistance. All remaining power settings achieve significantly higher fluences for a potentially better phase transformation. The same argument holds true for the chosen velocities. A setting of $50 \mu \mathrm{m} / \mathrm{s}$ has been used before and did not yield conducting channels. However, for this setting it has to be kept in mind, that the length of the focal plane is drastically reduced, so that the number of pulses with high intensity on the same spot is reduced even for this setting with a slow velocity. This is important for the exit side, since less phase changed material might be evaporated.

The success rate of phase changed material stretching through the whole diamond determined by optical inspection is shown in table 7.1. In this case failed channels are defined as channels, where the phase changed material does either not reach the exit or the seed side (28\% and $3 \%$ of the failed channels, respectively), the channel is interrupted in the bulk (62\%) or no phase change is observed at all (7\%). All channels grown at a velocity of $200 \mu \mathrm{m} / \mathrm{s}$ or slower extend without interruptions fully through the sensor, according to optical inspection. The fraction of good channels decreases with higher velocities. For these velocities, the success rate drops with decreasing power. An explanation for this is the length of the phase changed region per pulse. For low velocities, the phase change happens upstream of the focal region, where the fluence is barely high enough to initiate phase transformation. Accordingly, the fluence in this region is independent of the power of the laser, as the power only influences the distance of this region with respect to the focal region. Once the velocity is sufficiently high, in other words the movement of the focal plane between two pulses is larger than the length of phase change at the low fluence threshold, the power of the laser actually determines the fluence at the region of phase change. The closer this region is to the focal plane, the higher the fluence is and the longer the phase changed region is. This yields a continuous channel as long as the length of the phase change per pulse is equally long as the movement of the focal plane per pulse. For the combination of high velocity and low power, this is not anymore the case. The channel grows so slow, that the interface between the phase changed material and diamond can slide downstream of the focal area. As the fluence in the focal area is high enough to cause spontaneous phase change, the channel is interrupted. 
7. $3 D$ Electrodes in detector grade $p C V D$ Diamond

\begin{tabular}{rr|r}
\hline Power $[\mathrm{mW}]$ & Velocity $[\mu \mathrm{m} / \mathrm{s}]$ & Success rate $[\%]$ \\
\hline 10 & 50 & 100 \\
10 & 200 & 100 \\
10 & 2000 & $20 \pm 18$ \\
10 & 10000 & $30 \pm 14$ \\
\hline 25 & 50 & 100 \\
25 & 200 & 100 \\
25 & 2000 & $68 \pm 7$ \\
25 & 10000 & $60 \pm 22$ \\
\hline 60 & 50 & 100 \\
60 & 200 & 100 \\
60 & 2000 & $95 \pm 3$ \\
60 & 10000 & $80 \pm 18$ \\
150 & 50 & 100 \\
150 & 200 & 100 \\
150 & 2000 & 100 \\
150 & 10000 & $90 \pm 9$ \\
\hline
\end{tabular}

Table 7.1.: Success rate by optical inspection of the channels grown in the first batch.

A second batch is produced in order to study the influence of the time of exposure to the femtosecond laser on the exit side. The observed crater like structure on the exit side shows that parts of the phase changed material are evaporated. The fluence threshold of the evaporation is unknown, but could be well below the threshold for phase change. This means, that the phase changed material is still evaporated, even if the focal plane is far upstream. In this case, the local fluence is too low to cause additional phase change, which leaves only unchanged diamond behind. After production, this could be compensated by focusing the beam once again onto the exit side. The idea is, that due to the high fluence in the focal area, phase changed material is evaporated, but at the same time new diamond sections are phase changed. This may result in more phase changed material on the exit side than without this repeated irradiation.

For this second batch, laser powers of $60,100,150 \mathrm{~mW}$ and velocities of $2,10 \mathrm{~mm} / \mathrm{s}$ are used. Twenty channels for each parameter combination are grown. For ten of these, after production the focal plane is again focused on the exit side, while the shutter is closed. The shutter is then opened in order to reirradiate the channel. The steering of the shutter does not allow to open it for a defined time. It can only be opened while at least one of the axes is moving. Accordingly, a minor movement along the beam axis is programmed in order to open the shutter. This movement is far below the step size of the stage, so that the shutter should close immediately after being opened. The 


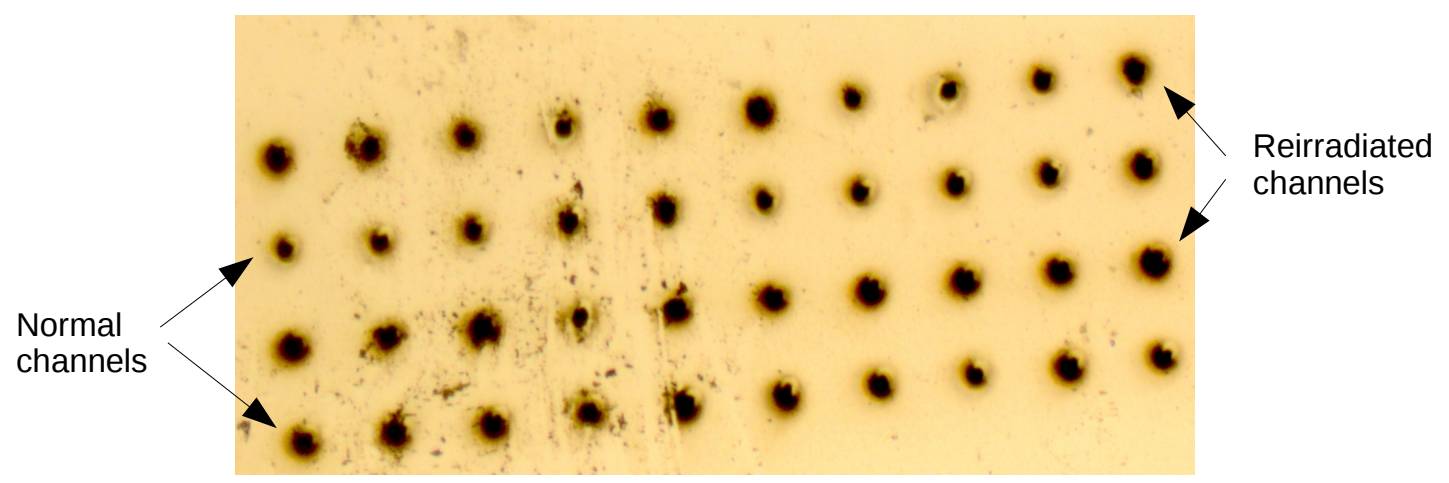

Figure 7.2.: Picture of the exit side of channels grown with a laser power of $100 \mathrm{~mW}$. The lower two rows were produced with a sample velocity of $2 \mathrm{~mm} / \mathrm{s}$, the upper two rows with $10 \mathrm{~mm} / \mathrm{s}$. For every second row, the exit side was reirradiated for about $50 \mathrm{~ms}$.

opening time of the shutter is estimated to be in the order of $50 \mathrm{~ms}$ which equals 10,000 pulses. A picture of the exit side of channels from the second batch is shown in figure 7.2.

For all of the 120 channels a continuous phase change expanding through the whole diamond is observed. This success rate is in agreement with the rates of the first batch. On the exit side dark material around the channels can be observed. This could also be seen for all previous produced channels. The diameters of the ejecta depend in first order on the used laser power. But they can also differ significantly between two channels with the same setting. For reirradiated channels, a slightly larger diameter is observed.

\subsection{Raman Spectroscopy}

For the Raman spectroscopy of this diamond sample, the same setup is used as described in section 6.6. In case of the channels produced in batch one, for each parameter combination, spectra of at least five channels are recorded on the seed side. After metallisation, each of these channels can be contacted individually. Spectra of the seed side for channels grown with a power of $10 \mathrm{~mW}$ and a velocity of $200 \mu \mathrm{m} / \mathrm{s}$ are shown in figure $7.3(\mathrm{a})$ and for $60 \mathrm{~mW}$ and $2 \mathrm{~mm} / \mathrm{s}$ in figure $7.3(\mathrm{~b})$. The spectra are normalised to the amplitude of the g-peak. It can be seen that for the low speed and power combination a dominant diamond peak is present, whereas for the high laser power and sample velocity combination, the diamond contribution is for some channels nearly invisible. Accordingly, the contribution of phase changed material is small compared to the diamond peak for the first parameter combination. The dominant diamond peak is partly caused by the very small diameter of these channels, which is in the order of $2 \mu \mathrm{m}$. Assuming a diameter of the laser of $1 \mu \mathrm{m}$, which is the upper limit measured in section 6.6 , about $37 \%$ of the Raman laser does not hit the channel but the surrounding diamond [111]. Still, the phase changed material of the channel is much more sensitive to Raman scattering than 
7. 3D Electrodes in detector grade $p C V D$ Diamond

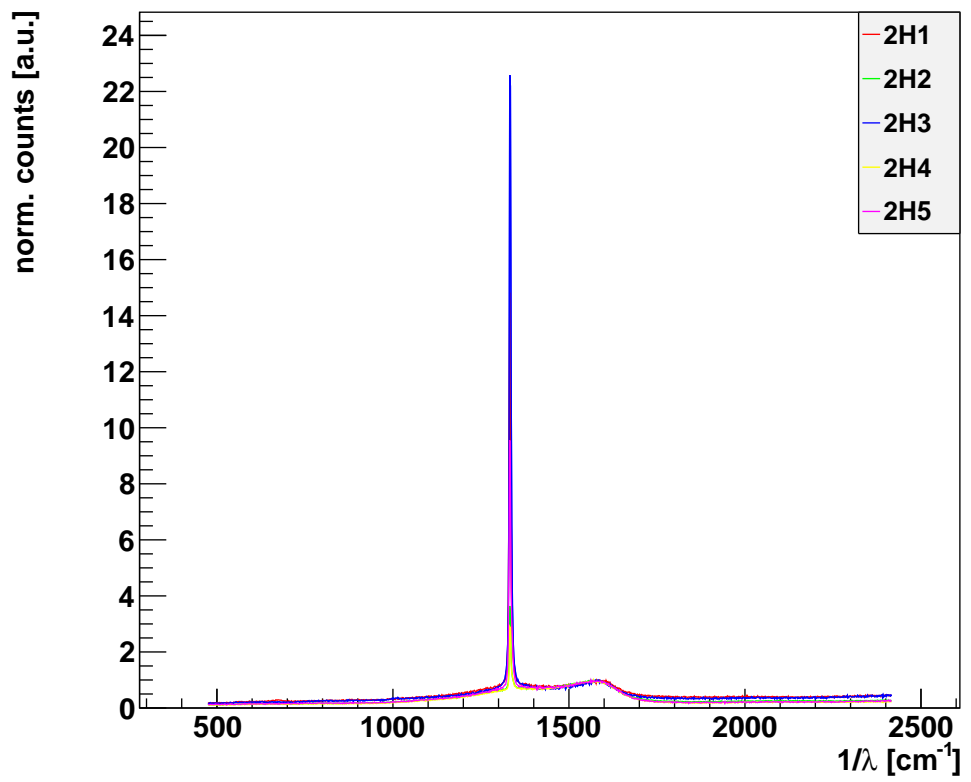

(a)

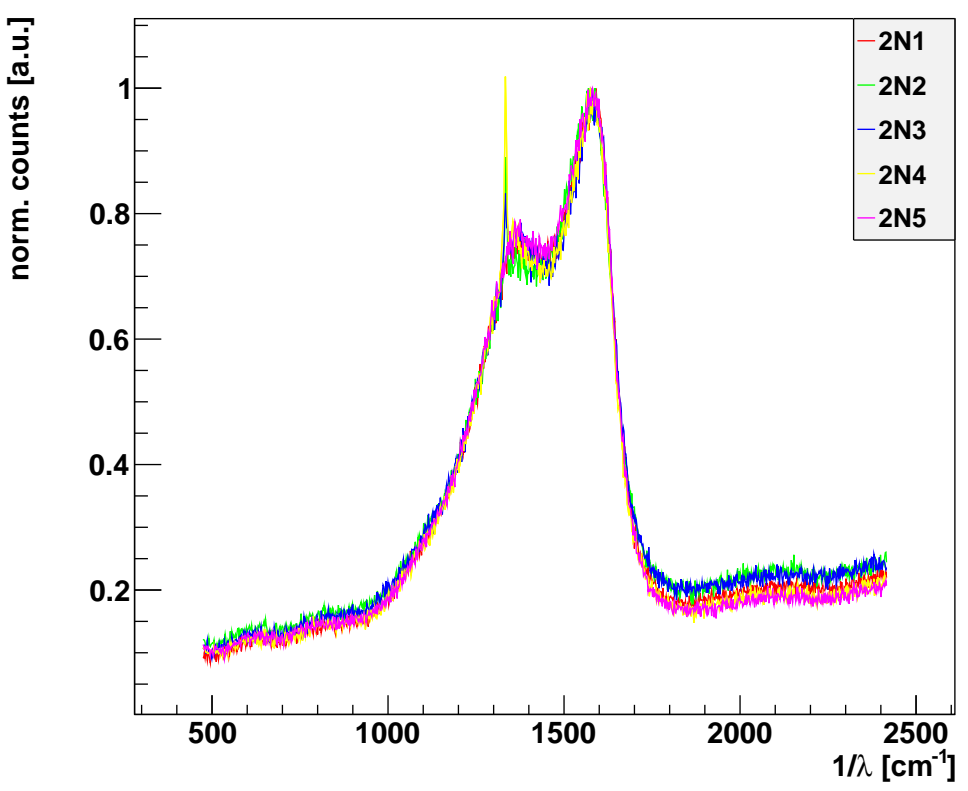

(b)

Figure 7.3.: Raman spectra of the seed side for channels produced with (a) $10 \mathrm{~mW}$ and $200 \mu \mathrm{m} / \mathrm{s}$ and (b) $60 \mathrm{~mW}$ and $2 \mathrm{~mm} / \mathrm{s}$. The intensity is normalised to the g-peak. Individual channels are indicated by different colours ${ }^{a}$.

${ }^{a}$ Channels grown with the same settings are indicated by having the same leading number and letter. 
the diamond [112]. Therefore, the spectra confirm the insufficient phase transition as measured with Goe-pCVD-02 before. Such spectra with a dominant diamond contribution are observed for channels produced at a power of $25 \mathrm{~mW}$ or less or at a velocity of $200 \mu \mathrm{m} / \mathrm{s}$ or less. This is in good agreement with the results shown in section 6.6.

For the parameter combination of $60 \mathrm{~mW}$ and $2 \mathrm{~mm} / \mathrm{s}$ the amplitude of the diamond peak in the spectra decreases significantly with respect to spectra for channels grown at a power of $10 \mathrm{~mW}$ and a velocity of $200 \mu \mathrm{m} / \mathrm{s}$. The peak is now only minor compared to the very visible d- and g-peaks. For two channels it is even completely invisible. This decrease of the amplitude of the diamond peak compared to g-and d-peak shows that at this higher laser power more atoms change their phase. Therefore, an increased conductivity of these channels on the seed side can be expected. Comparing the spectra with the ones measured for the graphite sticks in section 6.6, shows that the d- and the g-peak of the channels are not as well separated as for the graphite sticks. Especially the d-peak, which arises from disordering of the crystal, is broader. This hints at a lower conductivity of the channels compared with the graphite sticks [113]. The shape of the $s p^{2}$ contribution, i.e. the relative amplitudes of the d- and g-peak and their width, remains the same, regardless of the magnitude of the diamond peak. As the diameter of the channel is in the order of $4 \mu \mathrm{m}$ this diamond peak might also be caused by the surrounding material.

Furthermore, for channels grown with a laser power of $60 \mathrm{~mW}$ and $150 \mathrm{~mW}$ the spectra of the exit side are measured. Channels produced with a lower power show already on the seed side a minor contribution of phase changed material only, see figure B.1 - B.4. On the exit side this contribution is negligible compared to the diamond peak, as already seen for Goe-pCVD-02. Therefore, the spectra of the exit side for channels produced with a power of $10 \mathrm{~mW}$ or $25 \mathrm{~mW}$ are not measured. For the parameter combination of a laser power of $60 \mathrm{~mW}$ and a sample velocity of $2 \mathrm{~mm} / \mathrm{s}$, spectra for six additional channels from the large grid are recorded.

The spectra measured on the exit side show for velocities of $2 \mathrm{~mm} / \mathrm{s}$ or faster a significant non-diamond contribution. An example of this is shown in figure 7.4 for channels produced at a power of $150 \mathrm{~mW}$ and a velocity of $2 \mathrm{~mm} / \mathrm{s}$. The relative intensity of the diamond peak is nearly the same on both sides. The difference is the relative intensity of the d-peak, which is higher on the exit side. The g- and d-peaks are also better separated. Still, the amount of $s p^{2}$ contribution in the spectra hints at an increased conductivity of these channels.

In order to quantify the quality of the phase changed material, every spectrum is fitted by a function consisting of five Gaussian distributions and a linear function for the background. An example is shown in figure 7.5. In literature a wide variety of functions describing Raman spectra of carbon films can be found [114]. Most of the time these use either Gaussian or Lorentzian distributions (or both) and two to four peaks. For 
7. 3D Electrodes in detector grade $p C V D$ Diamond

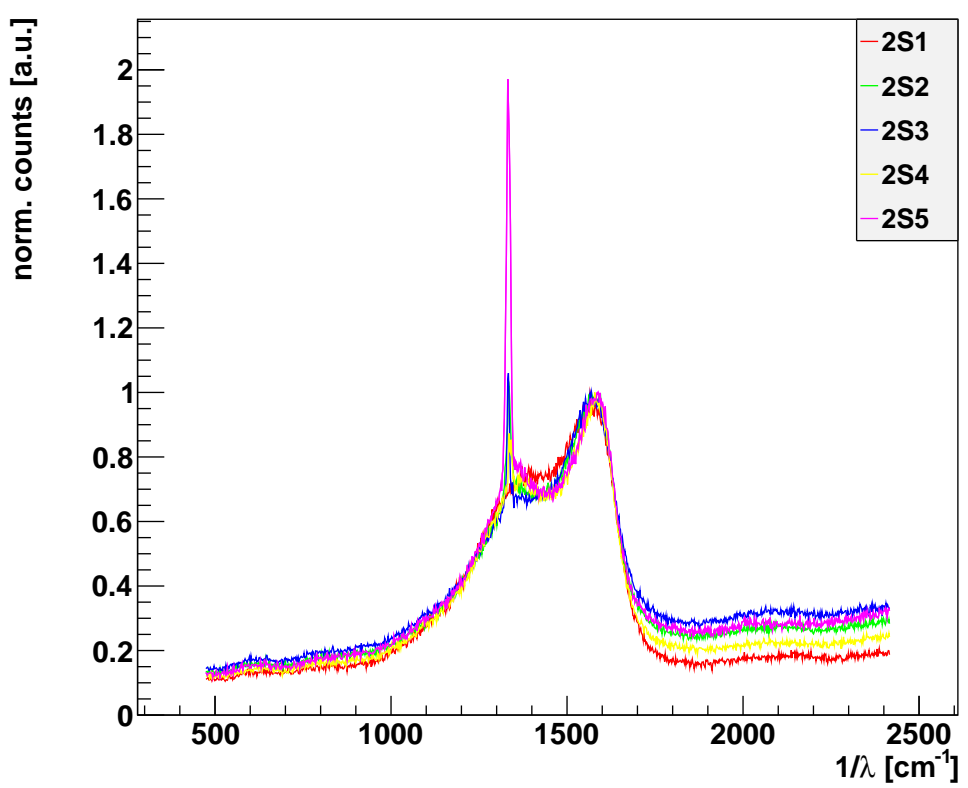

(a)

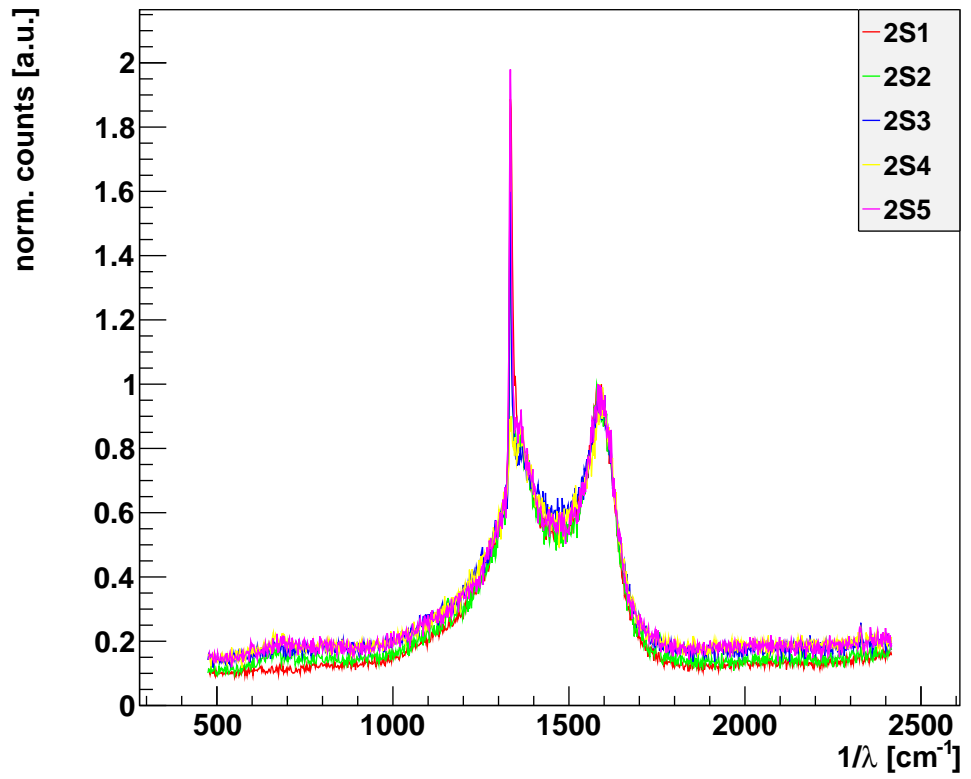

(b)

Figure 7.4.: Raman spectra for channels produced with $150 \mathrm{~mW}$ and $2 \mathrm{~mm} / \mathrm{s}$ for (a) the seed side and (b) the exit side. The intensity is normalised to the g-peak. Individual channels are indicated by different colours. 


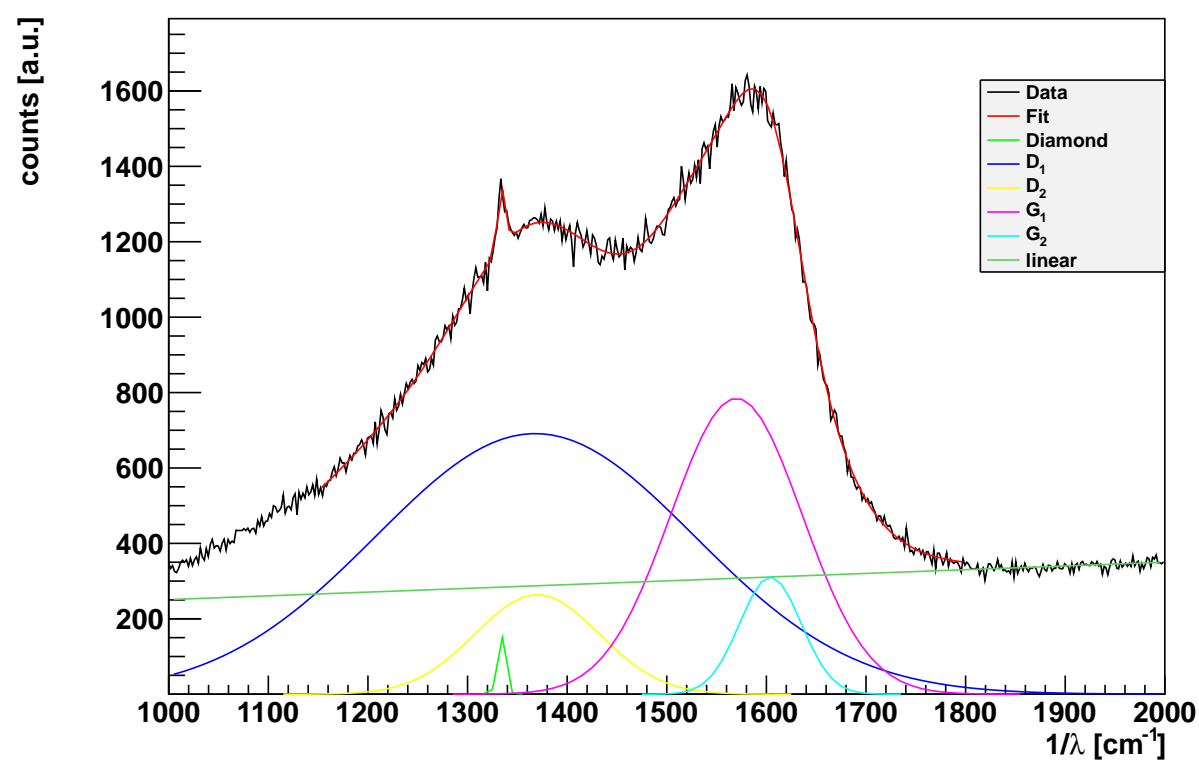

Figure 7.5.: Fit on the Raman spectrum of the seed side of a channel produced at $60 \mathrm{~mW}$ and $2 \mathrm{~mm} / \mathrm{s}$. The g- and d-peak are fitted using two Gaussian distributions each $\left(G_{1,2}\right.$ and $D_{1,2}$, respectively. One Gaussian distribution is fitted onto the diamond peak and the background is fitted with a linear function.

this thesis, four Gaussian distributions as fitting functions on the d- and g-peak and one Gaussian for the diamond peak are chosen.

The slope of the linear function is determined by fitting it to the spectra in the region starting at $1800 \mathrm{~cm}^{-1}$ until $2400 \mathrm{~cm}^{-1}$. In this region no signal is observed. The fit on the signal is performed in the region between $1150 \mathrm{~cm}^{-1}$ and $1800 \mathrm{~cm}^{-1}$. The mean values of the five Gaussian distributions are not fixed in the signal fit, but are only allowed to vary within a certain range. These limits are given in table 7.2. The whole function is fitted at once to the spectra with the linear function being fixed to the value obtained by the background fit. Out of the spectra of the 124 measured channels, the fit converges for 117. As the width of the diamond peak is only about five data points, the fit on its amplitude is generally poor. For this reason, the diamond peak is fitted again with a Gaussian distribution to determine its amplitude.

A figure of merit for the quality of the phase change is the ratio of the amplitude of the diamond peak to the amplitude of the g-peak. The amplitude of the diamond peak is determined by the extra fit described above. For the amplitude of the g-peak, the Gaussian distributions $\mathrm{G}_{1}$ and $\mathrm{G}_{2}$ are summed up and their peak amplitude is determined. Its uncertainty is taken from the fit, with the correlations between the fit parameters taken into account. The main component of the uncertainty of the ratio is 
7. 3D Electrodes in detector grade $p C V D$ Diamond

\begin{tabular}{r|rr}
\hline Peak & Lower limit $\left[\mathrm{cm}^{-1}\right]$ & Upper limit $\left[\mathrm{cm}^{-1}\right]$ \\
\hline Diamond & 1331 & 1336 \\
$\mathrm{D}_{1}$ & 1300 & 1370 \\
$\mathrm{D}_{2}$ & 1320 & 1400 \\
$\mathrm{G}_{1}$ & 1450 & 1580 \\
$\mathrm{G}_{2}$ & 1580 & 1610 \\
\hline
\end{tabular}

Table 7.2.: Limits for the mean values of the five Gaussian distributions used to fit the Raman spectra.

the amplitude of the diamond peak, due to its limited resolution. As a lower amplitude of the diamond peak decreases the uncertainty on its value, the mean ratio of a setting is heavily influenced by spectra with a low amplitude of the diamond peak. Therefore, the variance of the individual channels from the mean value of a setting is calculated. Figure 7.6 shows the ratios of the amplitudes with respect to different growth parameters.

For the channels grown at a laser power of $10 \mathrm{~mW}$, very high ratios, up to values of 73 , are observed with large variance for individual channels on the seed side. This low power is comparable to the power values used for all the other diamond samples described in chapter 6 . The high ratios are in good agreement with the ratios measured for Goe-pCVD-02 and indicate that the phase transformation of the diamond is insufficient.

On the seed side, channels grown at a velocity of $50 \mu \mathrm{m} / \mathrm{s}$, which is a setting comparable to the velocities used before, also show a higher ratio and variance than channels grown at higher velocities. Especially the large variance hints that the phase transition happens in this case very upstream of the focal plane at the fluence threshold for phase transformation. As described in section 5.5, the focal plane moves very slowly through the diamond, so that micro defects can occur inside the diamond, lowering the threshold for phase transformation. If this threshold is barely exceeded, the phase might be changed for enough atoms to absorb the laser beam, but not to yield a good conductive channel. As the formation of such micro defects is a statistical process, this can explain the large variance.

For channels which were produced at a laser power of at least $25 \mathrm{~mW}$ and sample velocity of at least $200 \mu \mathrm{m} / \mathrm{s}$, the ratio of the diamond amplitude to the amplitude of the g-peak is for almost all channels with values below 2 sufficiently low, so that a conductivity of the channel is possible. Especially channels grown at $60 \mathrm{~mW}$ or more and $2 \mathrm{~mm} / \mathrm{s}$ or faster have a very low ratio.

The influence of the velocity on the composition of the channels on the exit side is very apparent in the Raman spectra. For velocities up to $2 \mathrm{~mm} / \mathrm{s}$, the ratio decreases with increasing velocity. This is probably due to the reduced number of laser pulses on 


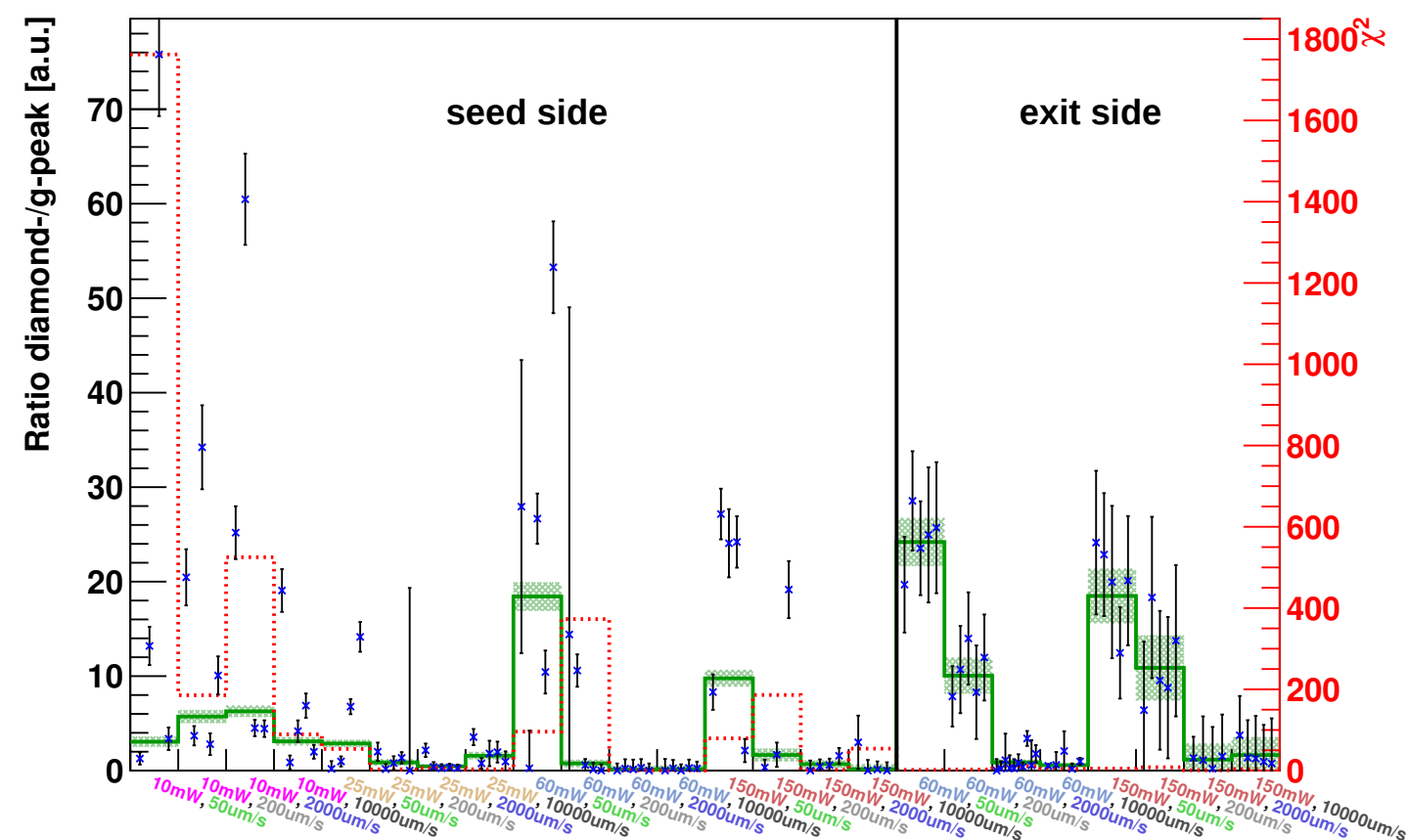

Figure 7.6.: Ratio of the amplitudes of the diamond peak with respect to the g-peak for different growth parameters. Blue crosses indicate measurements for single channels, green bins the mean value and its uncertainty for a setting. The $\chi^{2}$ for each setting is plotted in red to get a handle on the variance of each setting. 


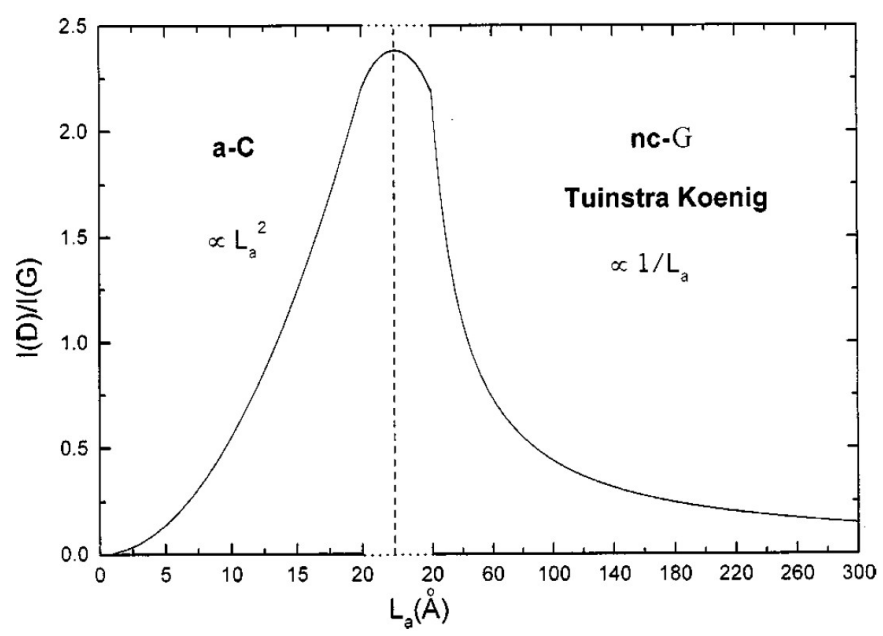

Figure 7.7.: Relation between the length of the $s p^{2}$ grains and the ratio of the amplitude of the d-peak to the amplitude of the g-peak $[109,115]$.

the exit side for higher velocities. Velocities of $2 \mathrm{~mm} / \mathrm{s}$ and $10 \mathrm{~mm} / \mathrm{s}$ do not significantly differ in the measured ratio, which is comparable to the ratios obtained for these channels on the seed side. Accordingly, an increased conductivity of these channels on the exit side is expected. The power of the laser of either $60 \mathrm{~mW}$ or $150 \mathrm{~mW}$ does not have a significant influence on the ratio.

According to F. Tuinstra and J.L. Koenig, the ratio of the amplitude of the d-peak to the amplitude of the g-peak can be used to determine the grain size of the $s p^{2}$ bound atoms, as shown in figure 7.7 [115]. The relation is ambiguous, so that it has to be known if the material is more like amorphous carbon or nanocrystalline graphite. Figure 7.8 shows the ratio of the amplitude of the d-peak to the amplitude of the g-peak for the measured channels. It can be seen that it is almost constant for most parameter combinations, except for channels on the exit side, which were grown with a velocity of $200 \mu \mathrm{m} / \mathrm{s}$ or less. For these channels the amplitude of the d-peak with respect to the g-peak is significantly higher than for the other settings. The change of this ratio is an indication, that the remaining material on the exit side for these channels has a different composition than the other channels [109]. A high spread in the measured ratios on the seed side is also observed for channels grown at $50 \mu \mathrm{m} / \mathrm{s}$ with most of the ratios being higher than the values measured for other settings. As mentioned, more information is needed in order to determine the size of a $s p^{2}$ grain. Settings, which have a higher ratio of the amplitude of the d-peak to the amplitude of the g-peak, typically also have a higher diamond contribution in the recorded spectra. Accordingly, their phase transformation should be not as complete as for channels with lower ratios. Therefore, their grain size should be smaller. This indicates that the phase changed material is nanocrystalline graphite. 


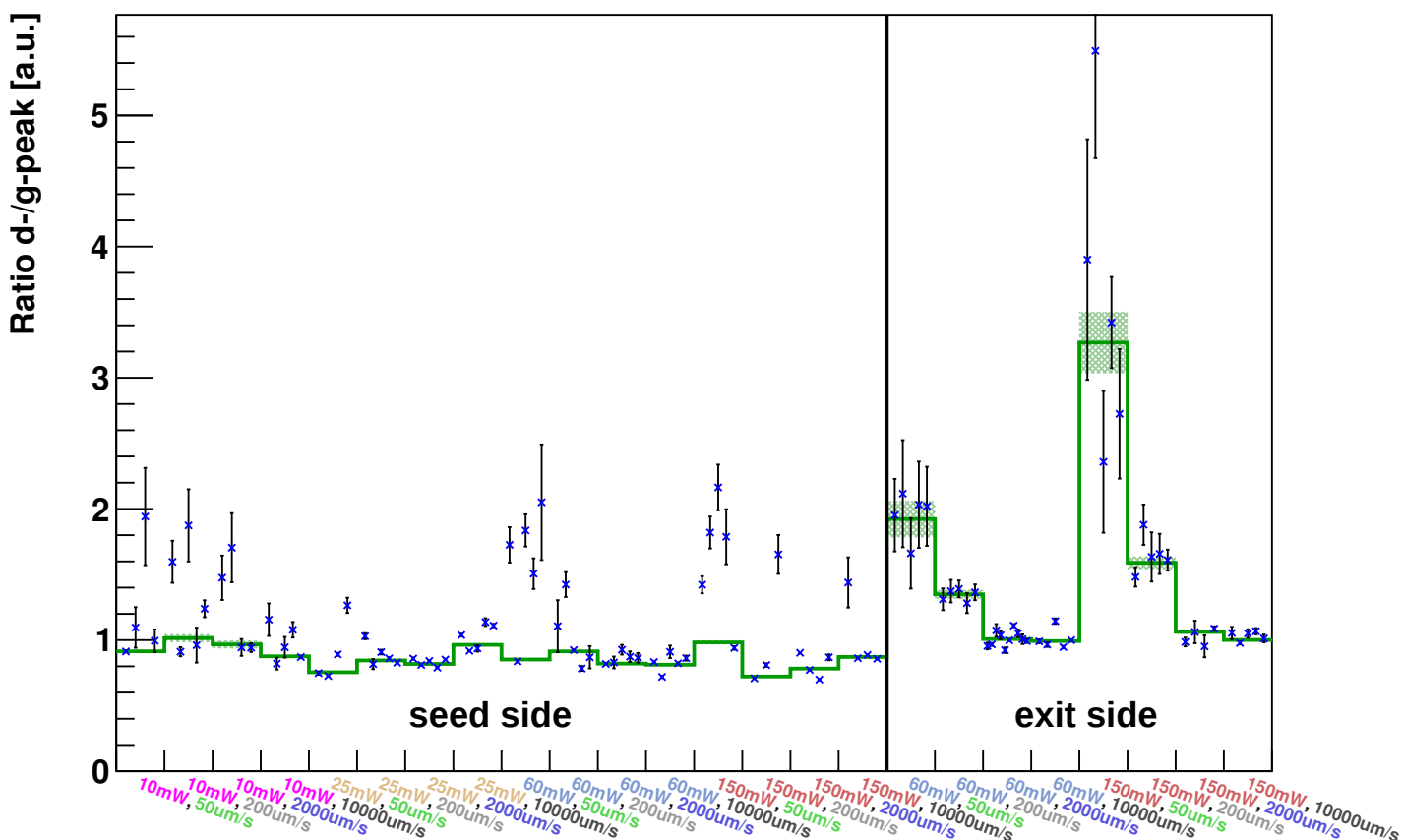

Figure 7.8.: Ratio of the amplitudes of the d-peak with respect to the g-peak. Blue crosses indicate measurements for single channels, green bins the mean value for a setting. 


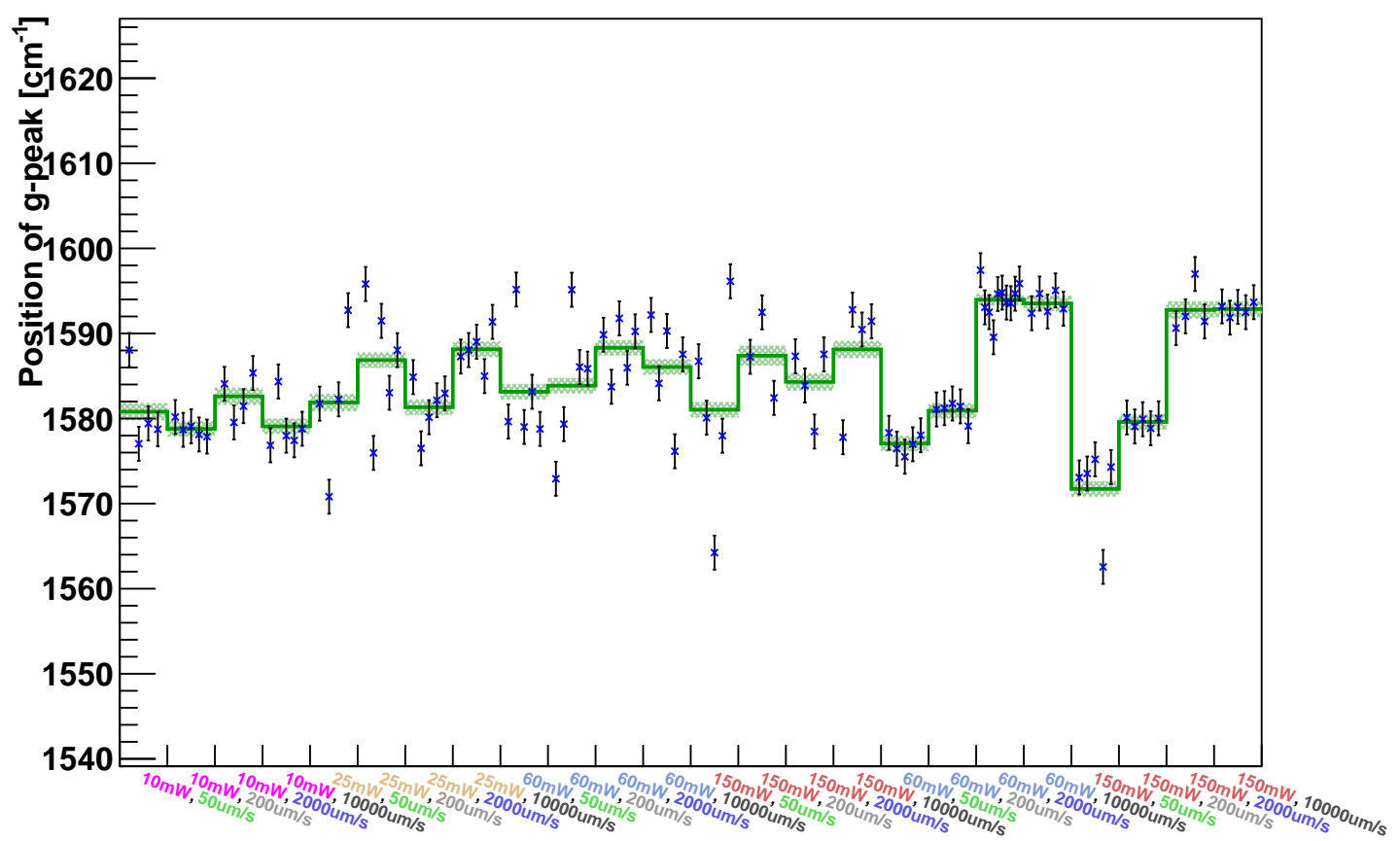

Figure 7.9.: Position of the g-peak. Blue crosses indicate measurements for single channels, green bins the mean value for a setting.

Another quantity which supports this hypothesis is the position of the g-peak. For a perfect graphite crystal it should be at $1581 \mathrm{~cm}^{-1}$ and for nanocrystalline graphite the peak position moves to approximately $1600 \mathrm{~cm}^{-1}[107,109]$. In case of amorphous carbon, the g-peak is located around $1510 \mathrm{~cm}^{-1}$ [109]. The position of the g-peak of the fitted function for different growth parameters is shown in figure 7.9. It can be seen, that for almost all channels it is located between $1570 \mathrm{~cm}^{-1}$ and $1600 \mathrm{~cm}^{-1}$.

With the hypothesis of nanocrystalline graphite, the mean grain size $L_{a}$ of the $s p^{2}$ bound atoms can be calculated via [115]

$$
L_{a}=C(\lambda) \frac{I_{g}}{I_{d}}
$$

with $I_{d, g}$ being the amplitude of the d- and g-peak, respectively. $C(\lambda)$ depends on the wavelength of the Raman laser and is for $488 \mathrm{~nm}$ approximately $3.5 \mathrm{~nm}$ [116]. Accordingly, for the seed side channels grown with a power of $25 \mathrm{~mW}$ or higher and a velocity of $200 \mu \mathrm{m} / \mathrm{s}$ or more crystal sizes between approximately $3.9 \mathrm{~nm}$ and $5.0 \mathrm{~nm}$ are observed. Most of the channels grown at a velocity of $50 \mu \mathrm{m} / \mathrm{s}$ only have crystal sizes of $1.8 \mathrm{~nm}$ to $2.3 \mathrm{~nm}$ on the seed side, which is disadvantageous for their conductivity. This is also observed on the exit side, where channels grown at a velocity of $200 \mu \mathrm{m} / \mathrm{s}$ have a crystal 


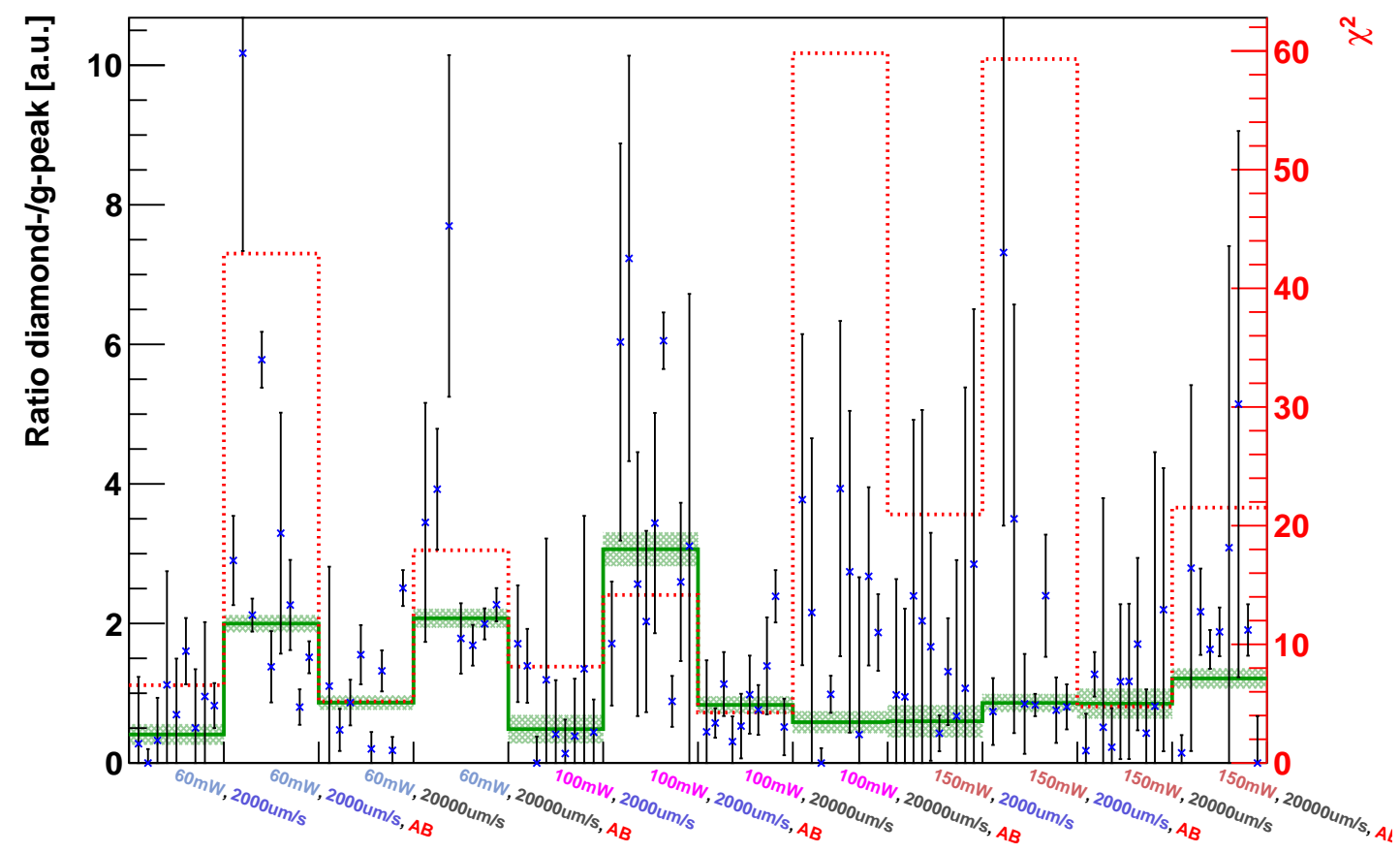

Figure 7.10.: Ratio of the amplitudes of the diamond peak with respect to the g-peak on the exit side. Blue crosses indicate measurements for single channels, green bins the mean value for a setting. The $\chi^{2}$ for each setting is plotted in red to get a handle on the variance of each setting. Channels, which have been reirradiated are indicated by "AB".

size of approximately $2.3 \mathrm{~nm}$ compared to $3.5 \mathrm{~nm}$ large grains for channels grown at a velocity of at least $2 \mathrm{~mm} / \mathrm{s}$. Therefore, channels which have a minor diamond contribution also have a larger grain size than channels with a dominant diamond peak.

From the Raman spectra it is suggested that channels grown with at least $60 \mathrm{~mW}$ and $2 \mathrm{~mm} / \mathrm{s}$ are conductive, as they have on the seed and exit side a low ratio of the amplitude of the diamond peak to the amplitude of the g-peak. However, the ratios on the exit side are still slightly higher than on the seed side. Therefore, the reirradiation of channels on the exit side is investigated with the third batch of channels. The ratios of the amplitude of the diamond peak to the amplitude of the g-peak are shown in figure 7.10 and the spectra of individual channels in section B.2. From the spectra of 118 channels, the fit converges for 108 channels. It can be seen that for all settings the measured ratio is reasonably low, with the results for channels without reirradiation being in agreement with the results obtained from the channels from the second batch. However, in all cases the reirradiated channels yield a higher ratio than channels of the same setting, which were not reirradiated. Further, the spread of the results, indicated by the value of $\chi^{2}$, is larger for reirradiated channels, which is considered unfavourable. 


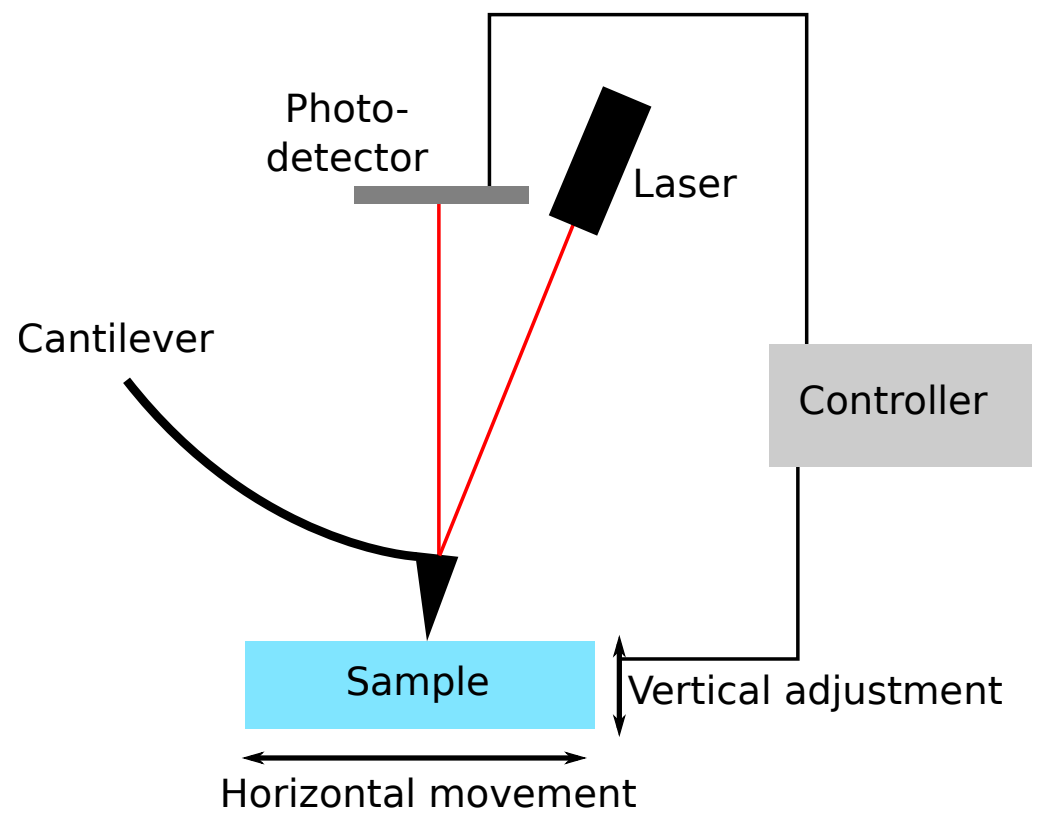

Figure 7.11.: Schema of an AFM measurement in contact mode. Note that the size of the tip of the cantilever and the roughness of the sample are not to scale.

This result disproves the hypothesis that a reirradiation is beneficial for the composition of the exit side.

\subsection{Atomic Force Microscopy}

The crater like structure on the exit side is critical for the contacting of the channel with a metal electrode. If the crater is too deep with respect to its diameter, the metal will not reach the bottom of the crater. In order to measure the structure of the crater, they are scanned with an Atomic Force Microscope (AFM). For this thesis an Asylum Research MFP-3D SA AFM located in the Institute of Materials Physics at the University of Göttingen is used.

A schema of the setup is shown in figure 7.11. The central component is a cantilever with a very fine tip. A picture of these can be seen in figure 7.12. The cantilever is a $160 \mu \mathrm{m}$ long, $40 \mu \mathrm{m}$ wide, and $3.7 \mu \mathrm{m}$ thick spring of silicon. At one end, it has a $14 \mu \mathrm{m}$ high tip, which has at its apex a radius of $9 \mathrm{~nm}$. If the AFM is operated in contact mode - also called constant force mode - this tip always touches the surface of the sample. The silicon cantilever is hereby slightly bend. The amplitude of this bending is measured using a laser, which is focused on the top of the cantilever, from where it is reflected onto a photo detector [118]. If the amplitude of the bending, i.e. the height of the surface of the sample, changes, the reflected laser spot moves across the photo detector. The vertical position of the sample is adjusted, so that the amplitude of the bending of the cantilever 


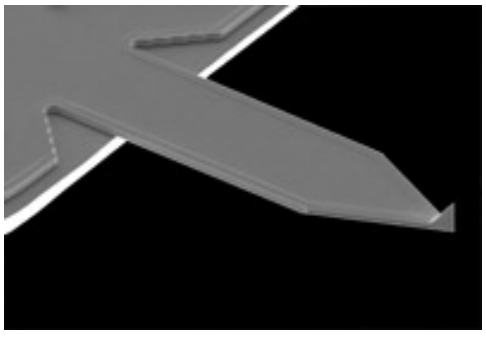

(a)

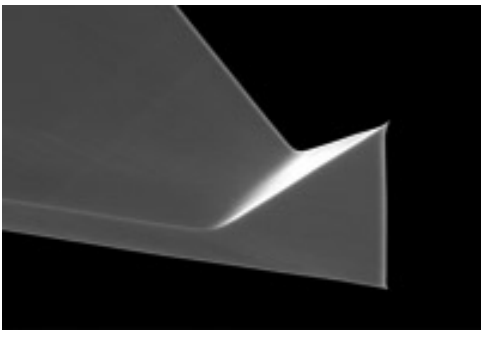

(b)

Figure 7.12.: Picture of (a) the cantilever and (b) a zoom on its tip [117]. The cantilever has a length of $160 \mu \mathrm{m}$, a width of $40 \mu \mathrm{m}$, and is $3.7 \mu \mathrm{m}$ thick. The tip has a height of $14 \mu \mathrm{m}$.

returns to its calibrated level. This adjustment is recorded and allows a relative height measurement of the surface of the sample.

In contact mode, soft structures can be modified by the movement of the tip [118]. For this reason, for the measurement of the phase changed material, the AFM is operated in tapping mode. In this mode the cantilever oscillates with a low amplitude, which is approximately $20 \mathrm{~nm}$ in our case. This oscillation is recorded by the photo detector by a movement of the reflected laser light. When the distance of the cantilever to the surface of the sample is smaller than the amplitude of the oscillation, the oscillation is dampened and its amplitude decreased. Therefore, a distance of the cantilever with respect to the surface of the sample is chosen, so that the amplitude of the oscillation is slightly decreased with respect to a free oscillation. If the height of the surface of the sample changes, its vertical position is adjusted, so that the amplitude of the oscillation is returned to its predefined value.

In order to measure the crater like structure of a channel, the sample is moved horizontally with respect to the cantilever. First, the sample is moved transversely with respect to the long direction of the cantilever. This direction of movement, called trace, slightly twists the cantilever in contact mode [118]. Therefore, in order to compensate this effect, the sample is moved back along the same route, which is called retrace. The height of the sample is then calculated by the arithmetic mean of trace and retrace heights. Although in tapping mode the cantilever is not twisted due to this movement, trace and retrace are still recorded. In a second step, the sample is moved along the long direction of the cantilever and the movement of trace and retrace is repeated.

Although the apex of the tip is very small, the tip itself has, as seen in figure 7.12(b), a pyramid shape, with dimensions in the order of the investigated structures. This shape influences the measurement, when moving across a steep edge. Due to the size of the tip, it might not touch the surface with its apex, but with its side. This results in 


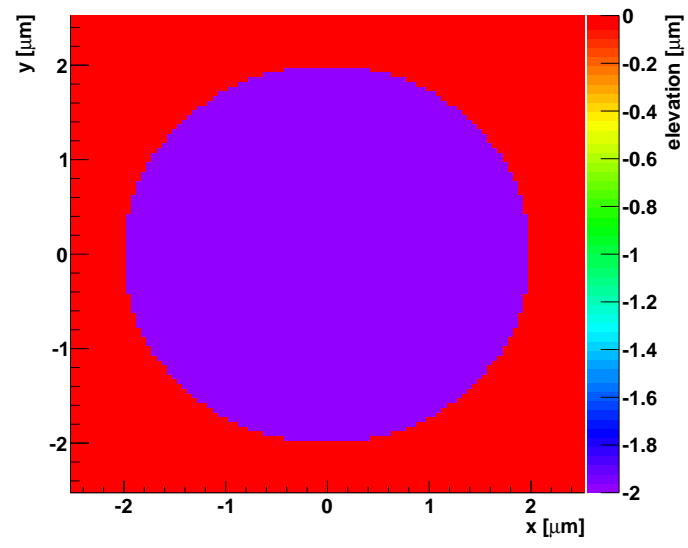

(a)

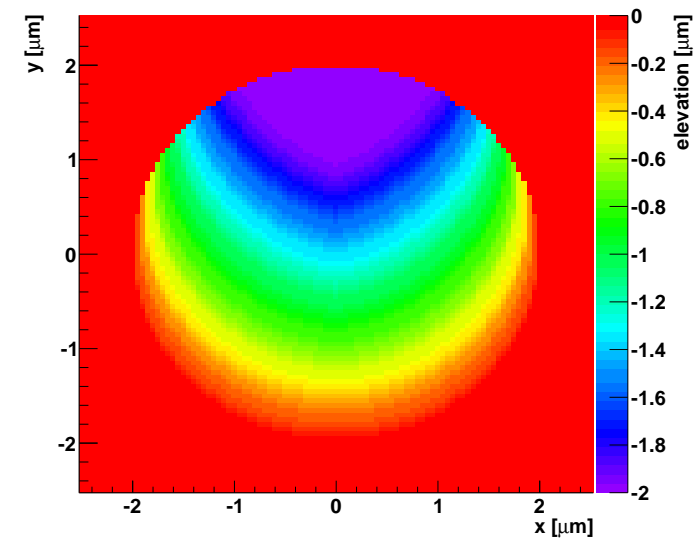

(b)

Figure 7.13.: Simulation of the effects of the size of the tip of the cantilever on the measurement of steep edges. The real surface is shown in (a) and the measured shape in (b).

mismeasurement of the actual shape of the surface. As the crater like structure on the exit side of the channels is expected to be steep, this effect is simulated. The result is shown in figure 7.13. It shows a circular structure with a diameter of $4 \mu \mathrm{m}$ and a depth of $2 \mu \mathrm{m}$. This structure is scanned from the bottom to the top with a tip of the same shape as the one used in the AFM. The tilted surface of the pyramid shape of the tip points towards the bottom. It has a side angle of $15^{\circ}$ and a back angle of $35^{\circ}$ [117]. The result shows that only the upper edge of the circular structure is measured accurately. For the lower half, the edge of the structure is hardly visible. It looks like a constant decrease in height and not like a sharp step.

A result of an AFM scan can be seen in figure 7.14(a), which shows the exit side of a channel produced with a laser power of $60 \mathrm{~mW}$ and a sample velocity of $10 \mathrm{~mm} / \mathrm{s}$. It is observed, that the resolution of the edge of the crater is better than in the simulation. This is due to an inclination of the cantilever with respect to the sample even if it does not touch the surface of the sample. The inclination of the cantilever reduces the inaccuracy of the measurement of the bottom edge shown in figure 7.13(b), but at the same time introduces an inaccuracy in the measurement of the upper edge. In total, the inclination of the cantilever increases the accuracy of the measurement. Figure 7.14(b) shows a height profile of the crater, starting at the lower left corner and ending on the upper right corner of the scan. The crater has a diameter of approximately $3.5 \mu \mathrm{m}$ and a depth of about $0.5 \mu \mathrm{m}$. This depth is reached across a distance of about $0.5 \mu \mathrm{m}$, although this measurement is limited by the physical dimensions of the tip. Nevertheless, the crater wall can be considered as very steep and challenging for metallisation. 


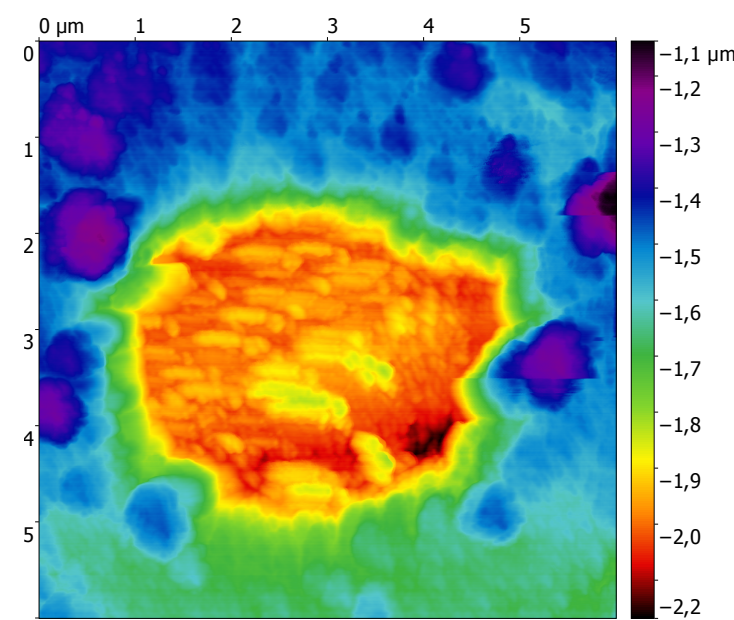

(a)

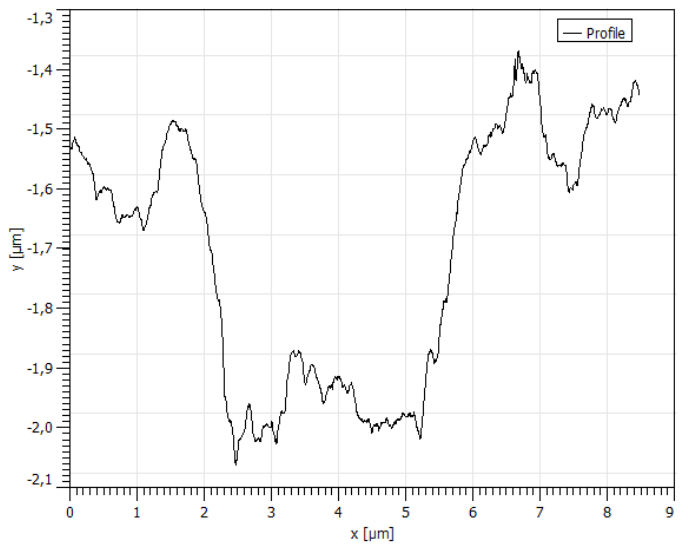

(b)

Figure 7.14.: (a) Height profile of the exit side of a channel grown with a laser power of $60 \mathrm{~mW}$ and a sample velocity of $10 \mathrm{~mm} / \mathrm{s}$ measured with an AFM. (b) Height profile of the crater along the diagonal from the lower left to the upper right corner.

The Raman spectra of the channels show a high variance of the amplitude of the diamond peak for some growth parameter combinations. For this reason, the exit sides of three channels grown at $60 \mathrm{~mW}$ and $10 \mathrm{~mm} / \mathrm{s}$ which have a very diverse diamond peak amplitude are scanned with an AFM. The pictures togehter with the respective Raman spectra are shown in figure 7.15. It can be seen that the lowest amplitude of the diamond peak is recorded for channel $2 \mathrm{O} 4$ shown in figure $7.15(\mathrm{f})$, which is the channel already presented in figure 7.14. For channel $2 \mathrm{O} 2$ the amplitude of the diamond peak is significantly higher, as can be seen in figure 7.15(b). The crater is deeper than channel 2O4, with a depth of about $1 \mu \mathrm{m}$. This is also reflected in the resolution of the crater walls. The plateau at the bottom of the crater seems to have a smaller area than for channel 2O4, but this is likely caused by the physical dimensions of the tip of the cantilever as the inclination is compareable. Out of the three channels, channel $2 \mathrm{O} 3$ has the highest relative amplitude of the diamond peak in the Raman spectrum. This is also reflected in the AFM scan of its surface. The diameter of the crater of channel $2 \mathrm{O} 3$ is comparable to the results for the other two channels. However, for most parts the crater is with only $0.2 \mu \mathrm{m}$ very shallow. The exception is a slit in the left half of the crater, which is with respect to the surrounding diamond surface $1 \mu \mathrm{m}$ deep. This slit covers an area of only $(1.5 \times 2.5) \mu \mathrm{m}^{2}$, which makes it very difficult for a metallisation to reach its bottom. 
7. 3D Electrodes in detector grade pCVD Diamond

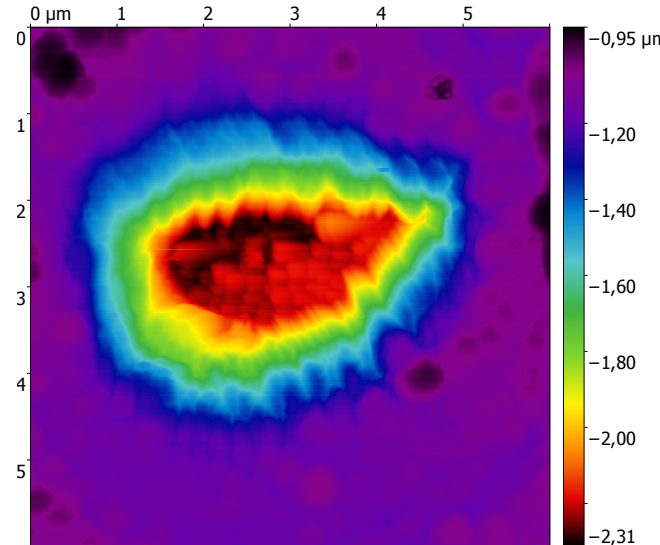

(a) Channel 2O2: AFM scan

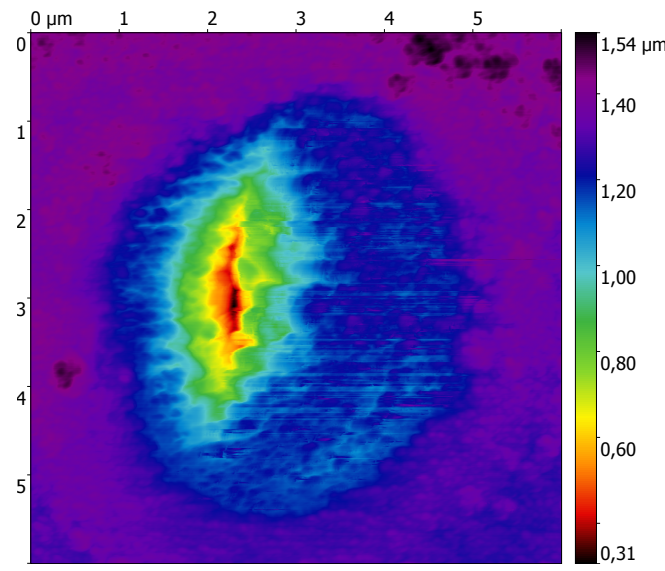

(c) Channel 2O3: AFM scan

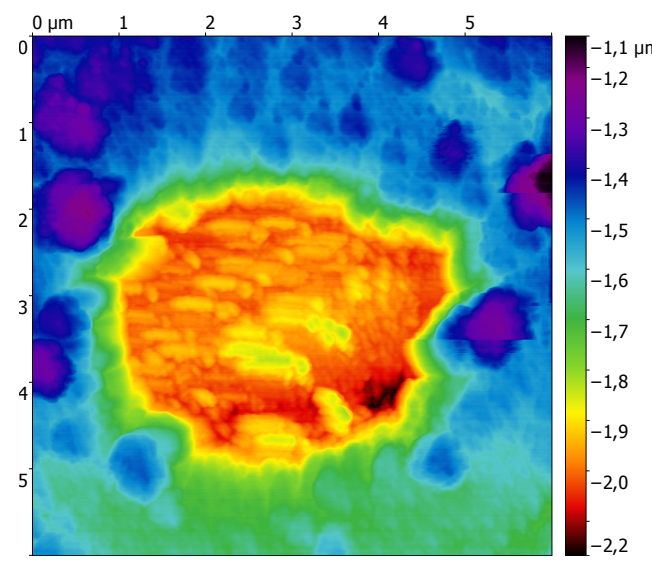

(e) Channel 2O4: AFM scan

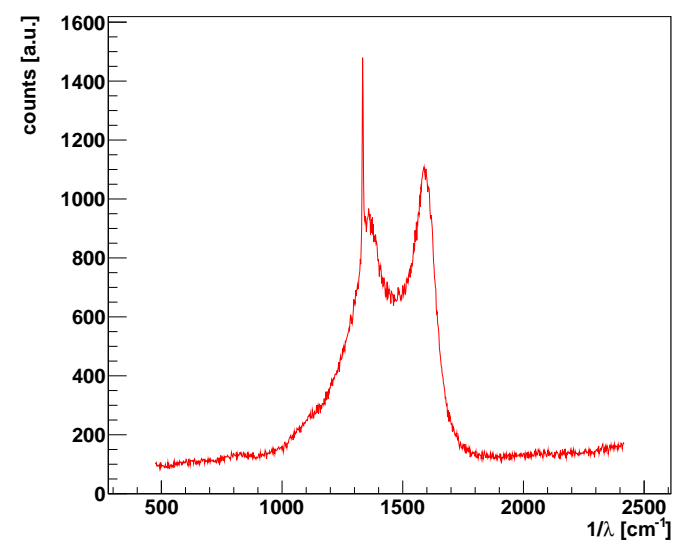

(b) Channel 2O2: Raman spectrum

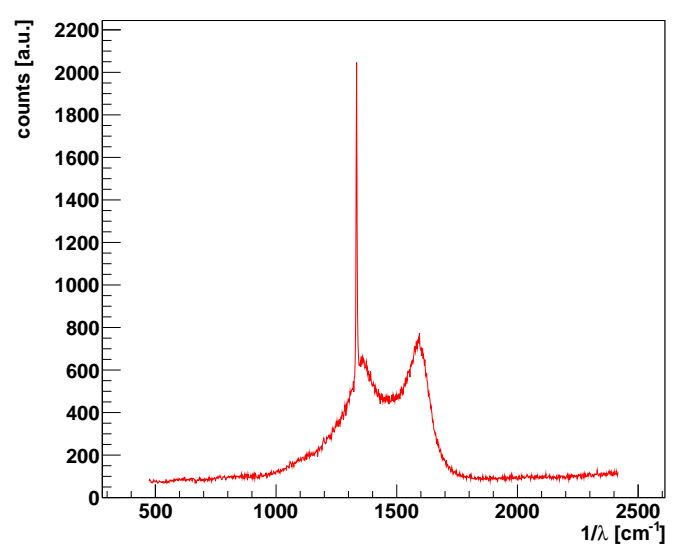

(d) Channel 2O3: Raman spectrum

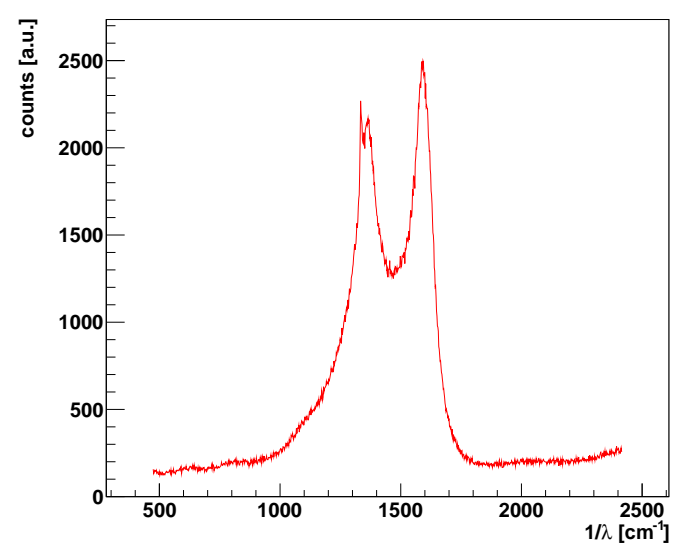

(f) Channel 2O4: Raman spectrum

Figure 7.15.: AFM scans of the exit side of three different channels grown at $60 \mathrm{~mW}$ and $10 \mathrm{~mm} / \mathrm{s}$ and their respective Raman spectra. 


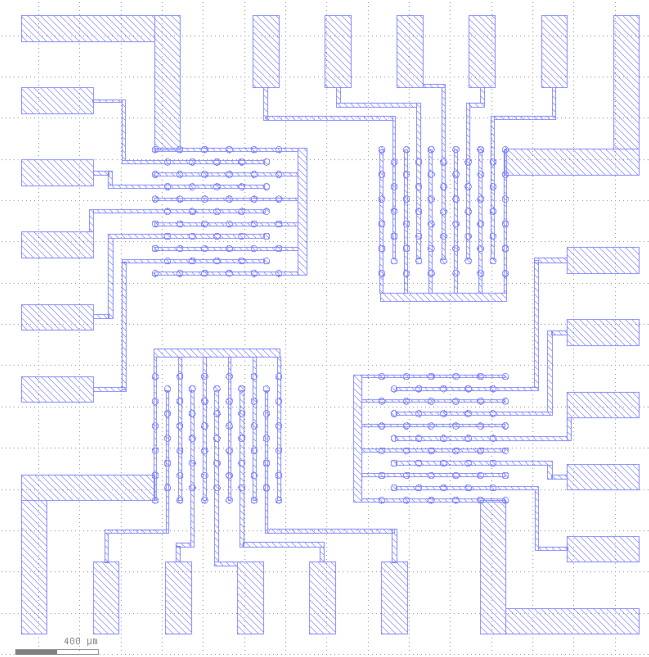

(a) Seed side

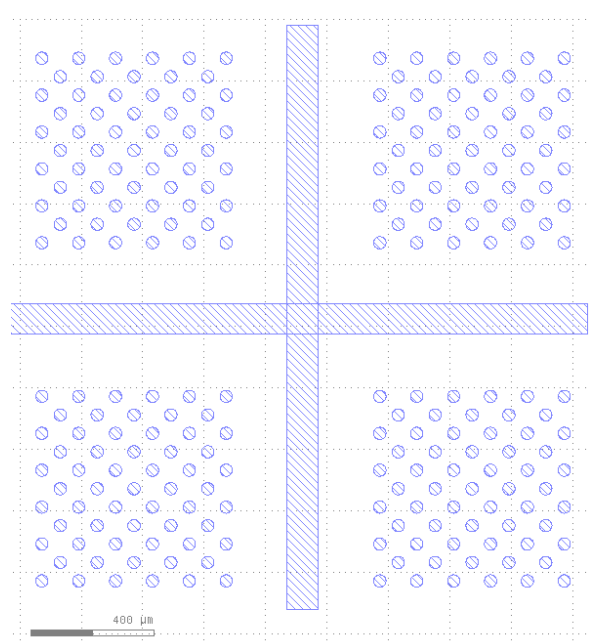

(b) Exit side

Figure 7.16.: Mask used for metallisation of the detector grade pCVD diamond sample. Note the slightly different scales.

\subsection{Resistivity Measurements}

The channels of the first batch were metallised at the University of Manchester with the mask shown in figure 7.16. The metallisation pattern on the exit side allows an individual contact via a probe needle for every channel. On the seed side each of the four six by six channels grid is connected with one strip like electrode. The small five by five channel grids are contacted via five strips, each of which connect five channels with the same setting. Each strip is routed to a large pad, which surround the four large grids.

In order to measure the resistance of the channels, the diamond sample is mounted on a PCB. The side with the strip electrodes is wire bonded to the PCB and connected to an Agilent 34410A multimeter. This multimeter is able to measure resistances of up to $1 \mathrm{G} \Omega$. The other side of the diamond sample is contacted via a needle. Contacting the pads of the strip electrodes with the needle yields a resistance of the measurement setup of under $4 \Omega$.

The resistance for all channels of the first batch grown with at least $60 \mathrm{~mW}$ of laser power are measured. Out of the eight growth parameter combinations in this batch, only for three combinations at least one channel has a resistance lower than $1 \mathrm{G} \Omega$. These parameter combinations are $60 \mathrm{~mW}$ and $2 \mathrm{~mm} / \mathrm{s}, 150 \mathrm{~mW}$ and $2 \mathrm{~mm} / \mathrm{s}$, and $150 \mathrm{~mW}$ and $10 \mathrm{~mm} / \mathrm{s}$. From their Raman spectra, these channels were assumed to be conductive. Their results are shown in figure 7.17 and in table 7.3. 


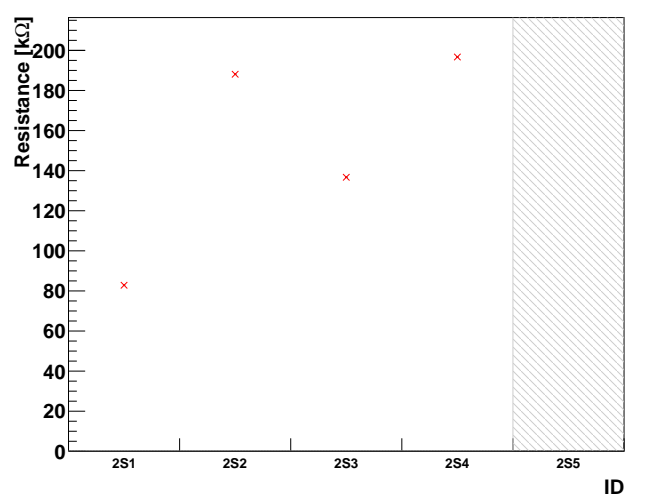

(a) $150 \mathrm{~mW}, 2 \mathrm{~mm} / \mathrm{s}$

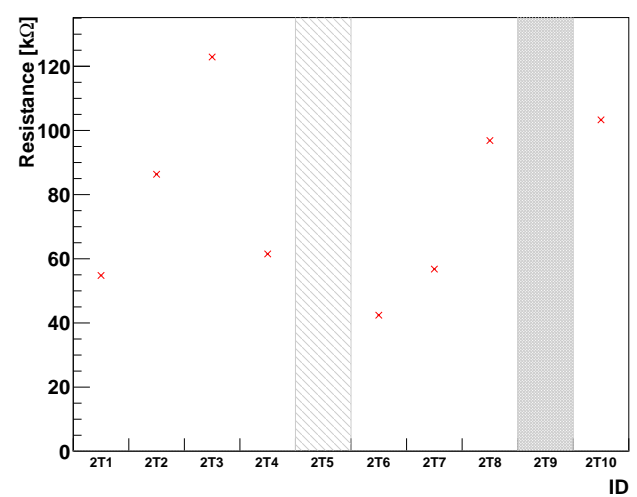

(b) $150 \mathrm{~mW}, 10 \mathrm{~mm} / \mathrm{s}$

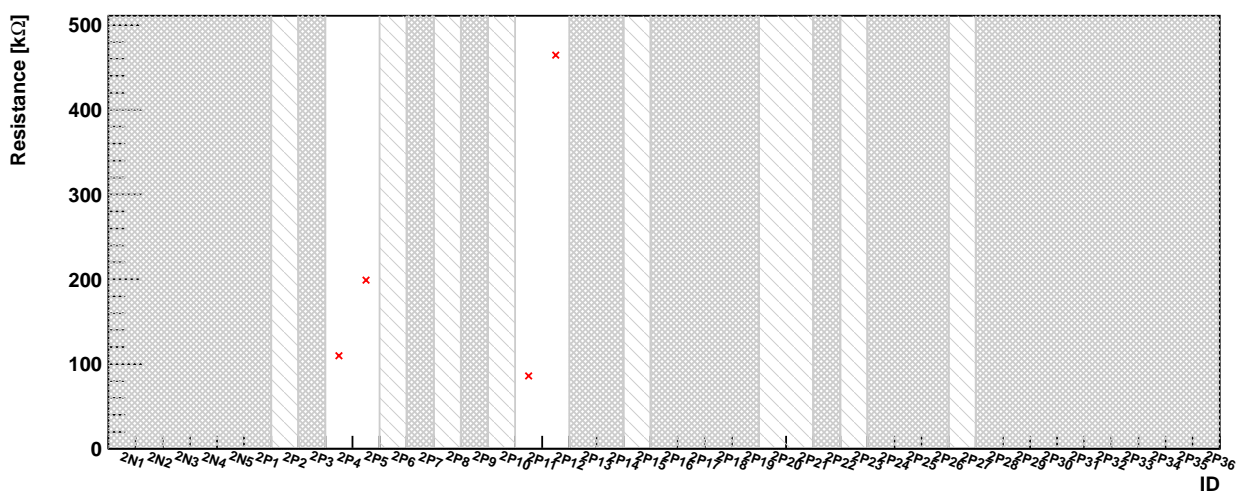

(c) $60 \mathrm{~mW}, 2 \mathrm{~mm} / \mathrm{s}$

Figure 7.17.: Results of the measurement of the resistance for channels produced with different growth parameters. A cut on the resistance for good channels of $1 \mathrm{M} \Omega$ is performed. Channels exceeding this value, but having a resistance below $1 \mathrm{G} \Omega$ are indicated by light grey bins, channels exceeding $1 \mathrm{G} \Omega$ by dark grey bins. The success rate for each growth parameter setting is given in table 7.3 .

\begin{tabular}{r|rrr}
\hline Growth parameters & Number of channels & $R<1 \mathrm{G} \Omega[\%]$ & $R<1 \mathrm{M} \Omega[\%]$ \\
\hline $60 \mathrm{~mW}, 2 \mathrm{~mm} / \mathrm{s}$ & 41 & $31.7 \pm 7.3$ & $9.8 \pm 4.7$ \\
$150 \mathrm{~mW}, 2 \mathrm{~mm} / \mathrm{s}$ & 5 & 100 & $80 \pm 18$ \\
$150 \mathrm{~mW}, 10 \mathrm{~mm} / \mathrm{s}$ & 10 & $90 \pm 9$ & $80 \pm 13$ \\
\hline
\end{tabular}

Table 7.3.: Fractions of channels for different growth parameters, which have a resistance $R$ lower than the indicated value. For all other combinations of growth parameters, the resistance of all channels is above $1 \mathrm{G} \Omega$. 


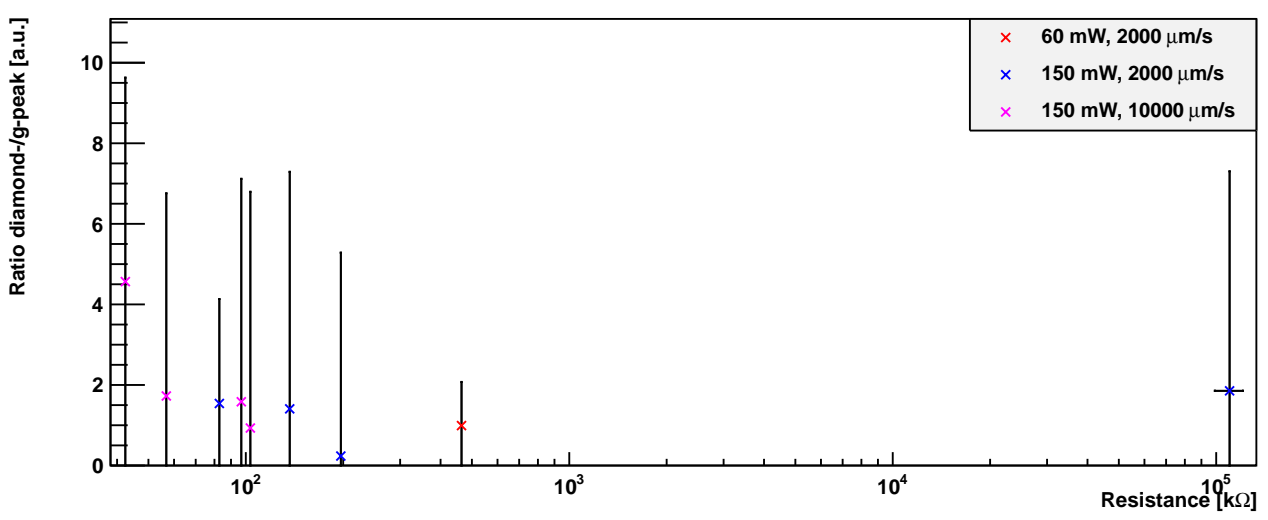

Figure 7.18.: Correlation between the ratio of the amplitudes of the diamond peak to the g-peak and the measured resistance.

Of the channels grown with a laser power of $150 \mathrm{~mW}$ and a sample velocity of at least $2 \mathrm{~mm} / \mathrm{s}, 80 \%$ show a resistance, which is below $1 \mathrm{M} \Omega$. In fact, for the resistance of channels grown at a velocity of $2 \mathrm{~mm} / \mathrm{s}$ and a power of $150 \mathrm{~mW}$ values between $80 \mathrm{k} \Omega$ and $200 \mathrm{k} \Omega$ for all channels except one are measured. The resistance of channels grown at a velocity of $10 \mathrm{~mm} / \mathrm{s}$ is even lower, with values between $40 \mathrm{k} \Omega$ and $125 \mathrm{k} \Omega$. For channels which have a measured resistance below $1 \mathrm{G} \Omega$ but above $1 \mathrm{M} \Omega$, typical values are in the order of $10^{\prime} \mathrm{s} \mathrm{M} \Omega$. Therefore, with both settings channels are produced, which are reasonably conductive, with a higher velocity yielding even better results.

Only a minority, namely $(31.7 \pm 7.3) \%$ of the channels grown with a laser power of $60 \mathrm{~mW}$ and a sample velocity of $2 \mathrm{~mm} / \mathrm{s}$ has a resistance below $1 \mathrm{G} \Omega$ and even fewer, namely $(9.8 \pm 4.7) \%$, a resistance below $1 \mathrm{M} \Omega$. Only four out of 41 channels are very conductive with values for the resistance between $100 \mathrm{k} \Omega$ and $500 \mathrm{k} \Omega$. This is a significantly higher resistance than for channels grown at a power of $150 \mathrm{~mW}$. Judging from the results of the Raman spectroscopy presented in section 7.3, a similar conductivity is expected for channels grown with a laser power of at least $60 \mathrm{~mW}$ and a velocity of at least $2 \mathrm{~mm} / \mathrm{s}$, as no significant differences in their spectra are observed. This is illustrated in figure 7.18, where the ratio of the amplitudes of the diamond peak to the g-peak with respect to the measured resistance is plotted. The ratios of the amplitudes of the diamond peak to the g-peak are for all channels below 5 and do not significantly differ due to the large uncertainty, but the resistance spans over three orders of magnitude. The large difference in the success rate between a laser power of $60 \mathrm{~mW}$ and $150 \mathrm{~mW}$ can therefore not be explained by differences in the composition of the phase changed material on the surface. A disruption of the channels inside the diamond bulk, which cannot be seen by optical inspection, is unlikely for such a huge number of channels. From the 41 channels grown at a power of $60 \mathrm{~mW}$ and a velocity of $2 \mathrm{~mm} / \mathrm{s}$ two of them have been identified to have a disruption in the bulk, from the five channels grown at a 


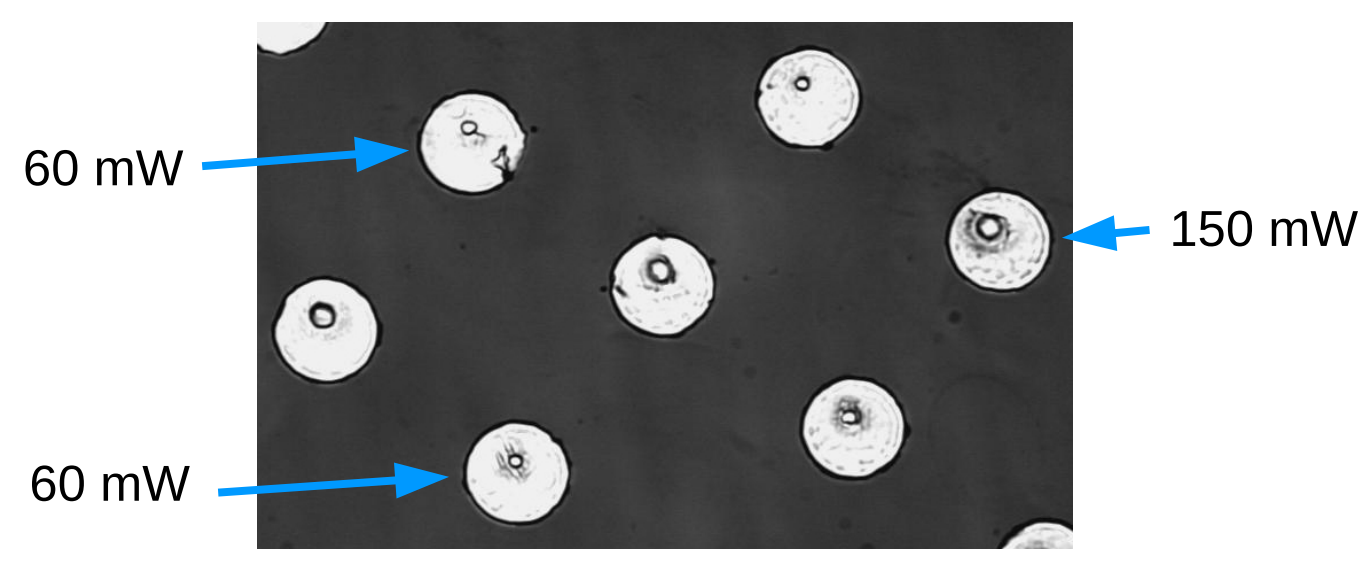

Figure 7.19.: Picture of the metallisation on the exit side of the channels. The three channels in the vertical centre of the picture are grown with a laser power of $150 \mathrm{~mW}$, the remaining four with $60 \mathrm{~mW}$. The differences in the diameter of the crater like structure can easily be seen.

power of $60 \mathrm{~mW}$ and a velocity of $10 \mathrm{~mm} / \mathrm{s}$ this is the case for only one channel. Furthermore, the resistance of more than $1 \mathrm{G} \Omega$ for all channels produced with a laser power of $60 \mathrm{~mW}$ and a sample velocity of $10 \mathrm{~mm} / \mathrm{s}$ can be explained by the low statistic of only four potential candidates ${ }^{1}$, assuming the same success rate measured for channels grown with a velocity of $2 \mathrm{~mm} / \mathrm{s}$.

As the composition of the phase changed material and the continuity of the channels are ruled out to cause the low success rate for channels grown at a laser power of $60 \mathrm{~mW}$, the problem might be caused by an insufficient contacting by the metal electrode. If the crater like structure on the exit side is too deep with respect to its diameter, the metal does not reach the bottom of the crater, which leads to an insufficient contact. With the metallisation on top of the channels, the diameter of the crater can easily be measured, as shown in figure 7.19. The mean values for the crater diameter for different growth parameters are given in table 7.4.

For laser powers of $60 \mathrm{~mW}$ and $150 \mathrm{~mW}$, a decrease in diameter with increasing velocity of the sample up to $2 \mathrm{~mm} / \mathrm{s}$ is measured. The larger diameter in case of a slower movement can be explained by the higher number of high intensity pulses on the exit side. This leads to a higher amount of ablation than for fewer pulses. Between velocities of $2 \mathrm{~mm} / \mathrm{s}$ and $10 \mathrm{~mm} / \mathrm{s}$ no significant difference in diameter is observed. The influence of the power is much higher than the influence of the velocity. Channels grown at a laser power of $150 \mathrm{~mW}$ are are about 1.5 times larger in diameter than channels grown at a laser power of $60 \mathrm{~mW}$.

\footnotetext{
${ }^{1}$ The channel shown in figure $7.15(\mathrm{e})$ has a disruption in the diamond bulk.
} 


\begin{tabular}{r|r}
\hline Growth parameters & Mean diameter $[\mu \mathrm{m}]$ \\
\hline $60 \mathrm{~mW}, 50 \mu \mathrm{m} / \mathrm{s}$ & $5.79 \pm 0.04$ \\
$60 \mathrm{~mW}, 200 \mu \mathrm{m} / \mathrm{s}$ & $5.30 \pm 0.02$ \\
$60 \mathrm{~mW}, 2 \mathrm{~mm} / \mathrm{s}$ & $4.78 \pm 0.02$ \\
$60 \mathrm{~mW}, 10 \mathrm{~mm} / \mathrm{s}$ & $4.81 \pm 0.07$ \\
\hline $150 \mathrm{~mW}, 50 \mu \mathrm{m} / \mathrm{s}$ & $8.63 \pm 0.08$ \\
$150 \mathrm{~mW}, 200 \mu \mathrm{m} / \mathrm{s}$ & $8.59 \pm 0.05$ \\
$150 \mathrm{~mW}, 2 \mathrm{~mm} / \mathrm{s}$ & $7.99 \pm 0.05$ \\
$150 \mathrm{~mW}, 10 \mathrm{~mm} / \mathrm{s}$ & $8.09 \pm 0.04$ \\
\hline
\end{tabular}

Table 7.4.: Mean values for the diameter of the crater like structure on the exit side with respect to the growth parameters.

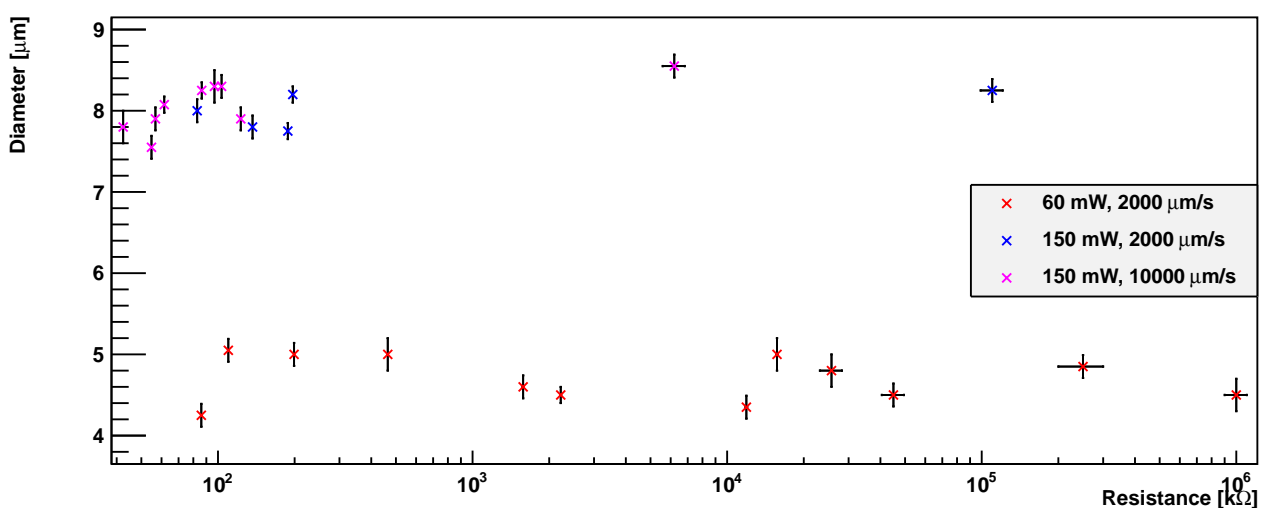

Figure 7.20.: Correlation between the diameter of the crater and the measured resistance.

The correlation between the diameter of the crater and the measured resistance of the channels is plotted in figure 7.20 for three different parameter settings. The diameter of the channels is nicely separated into groups of channels grown at a power of $60 \mathrm{~mW}$ and channels grown at a power of $150 \mathrm{~mW}$. The channels grown at a laser power of $150 \mathrm{~mW}$ cluster, with three exceptions ${ }^{2}$, at resistances below $200 \mathrm{k} \Omega$, whereas the resistances of channels grown at a laser power of $60 \mathrm{~mW}$ span from around $100 \mathrm{k} \Omega$ to $1 \mathrm{G} \Omega$. Most, namely $72 \%$, of the channels grown at a power of $60 \mathrm{~mW}$ and at least a sample velocity of $2 \mathrm{~mm} / \mathrm{s}$ even have a resistance above $1 \mathrm{G} \Omega$ and are not plotted.

Accordingly, the correlations between the ratio of the amplitudes of the diamond-peak and the g-peak, the diameter of the crater, and the resistance support the hypothesis of the insufficient contact of the metal electrode with the channel for small diameters.

\footnotetext{
${ }^{2}$ The resistance of one channel is not plotted, as it is above $1 \mathrm{G} \Omega$.
} 

CHAPTER 8

Conclusion \& Outlook

The LHC and its experiments, including the ATLAS detector, have shown an extraordinary performance in the past years, culminating in the discovery of the Higgs boson in $2012[15,16]$. In order to measure the properties of the Standard Model with a new level of precision and possibly discover physics beyond the Standard Model, more statistics is needed. This is achieved in the HL-LHC upgrade of the collider by increasing the instantaneous luminosity, which is planned for 2024. Since a higher instantaneous luminosity means a higher particle flux in the detector, with consequences for i.e. radiation hardness, read out bandwidth, and cost efficiency, a full replacement of the tracking detector is planned [31].

The innermost layer of this tracking detector is only few centimetres away from the interaction points of the particle beams and therefore has to withstand a huge particle flux, which causes radiation damage. One possible sensor candidate for this harsh environment is diamond, as it is intrinsically very radiation tolerant. After irradiation with doses of $10^{16}$ protons $/ \mathrm{cm}^{2}$ it performs, in terms of signal to noise ratio better than its competitor silicon [69]. The major challenge which has to be addressed when using diamond as a sensor material, is the trapping of charges. This trapping reduces the collected charge and therefore the signal. Especially cheaper diamonds suffer from a high probability of charge trapping.

In this thesis, a technique to use low quality polycrystalline diamonds as sensor material was presented, which is the utilisation of electrodes inside the diamond bulk, so called 3D electrodes [70]. These electrodes were produced using a femtosecond laser, which changes the phase of the diamond bulk material. It was observed, that a femtosecond laser reliably produces columns of phase changed material in the diamond bulk. Although it has a lower density than the surrounding diamond material, its induced me- 


\section{Conclusion \& Outlook}

chanical stress does not cause cracks in the diamond bulk. Even writing of channels, which are not perpendicular to the surface of the diamond, is possible.

The produced channels were found to have resistance in the order of the resistance of the diamond material. For this reason, Raman spectroscopy was performed, which showed that the phase change was insufficient. Therefore, the femtosecond laser setup was upgraded in order to allow higher fluences and higher velocities of the diamond sample. This new setup was tested with a detector grade diamond sample. The Raman spectra of the channels showed that the improved growth parameters significantly enhance the quality of the phase changed material. Especially on the exit side, a reduction of the remaining diamond material is achieved. Based on the spectra, the material is identified as nanocrystalline graphite, which is conductive.

The resistance of these channels was measured. Channels grown with the highest laser power of $150 \mathrm{~mW}$ and a sample velocity of at least $2 \mathrm{~mm} / \mathrm{s}$, show with values of less then $200 \mathrm{k} \Omega$ a sufficiently low resistance. For channels grown with a laser power of $60 \mathrm{~mW}$, a high resistance was measured, although they do not significantly differ in the Raman spectra. This is caused by the crater like structure on the exit side of the channels, which has a higher aspect ratio for lower powers. The structures on the exit sides were measured for some channels using an AFM, which showed that the used metallisation technique is not suitable for these structures.

This thesis revealed, that the production of conductive channels inside the diamond bulk is possible. The next step is the production of large areas of channels and connecting them to a read out chip. In order to test the performance of this sensor hybrid, it has to be evaluated in a testbeam. Meanwhile, other methods of metallisation like sputter deposition should be considered in order to contact the channels from the exit side more reliably [99]. The crater like structure on the exit side should also be minimised, e.g. by a transparent coating of the exit side. For the sensor production for a large detector, like the innermost layer of the tracker upgrade of the ATLAS detector, the production time and complexity is an important issue. The utilised femtosecond laser has a very high pulse frequency of $200 \mathrm{kHz}$, which greatly reduces the time needed to produce a channel compared to common $1 \mathrm{kHz}$ femtosecond lasers. In order to optimise the production process, beam splitters might be used to grow several channels at once. The overall complexity of a production of a $3 \mathrm{D}$ diamond sensor is due to its simple channel production and metallisation much lower than the complexity for 3D silicon sensors, which has a positive influence on the production costs. 
Zuerst möchte ich Arnulf Quadt dafür danken mir die Möglichkeit gegeben zu haben an diesem spannenden Thema in seiner Arbeitsgruppe zu forschen.

Im Rahmen dieser Arbeit wurden einige Geräte, welche nicht im II. Physikalischen Instituts verfügbar waren, benötigt, für deren Benutzung ich mich herzlich bedanken möchte. Insbesondere beim Laser Laboratorium Göttingen e.V. und hier speziell bei Thomas Fricke-Begemann, der mir bei der Bedienung des Femtosekundenlasers jedesmal sehr geholfen hat. Bei Burkhard Schmidt aus der Mineralogie des Geowissenschaftlichen Zentrums der Universität Göttingen bedanke ich mich für die Unterstützung bei der Ramanspektroskopie. Einen Dank auch an Hendrik Schmidt für die Hilfe bei den AFMMessungen.

Jens Weingarten gebührt mein Dank für die Unterstützung im Laufe der gesamten Arbeit. Die zahlreichen Ratschläge und Anregungen sowie aufbauenden Worte haben sehr geholfen. Für die gute Atmosphäre, beruflich wie privat, möchte ich mich bei der gesamten Hardwaregruppe, insbesondere bei Jörn, Jens, Julia, Matze, Johannes und Tobias bedanken. Auch allen anderen aktuellen wie ehemaligen Mitgliedern des II. Physikalischen Institutes einen herzlichen Dank für die gute Zeit und netten Gespäche.

Für das Korrekturlesen dieser Arbeit und die hilfreichen Anregungen einen großen Dank an Jens, Sabrina, Julia und Boris.

Meinen Freunden und meiner Familie danke ich für die Unterstützung während meines gesamten Studiums. Einen besonderen Dank an meine Eltern, die mir ein sorgenfreies Studium ermöglichten und mich seit Kindertagen mit ganzer Kraft und Liebe unterstützen.

\section{Danke!}



[1] J. Chadwick, The existence of a neutron, in Proceedings of the Royal Society of London A: Mathematical, Physical and Engineering Sciences, vol. 136, pp. 692-708, The Royal Society. 1932.

[2] M. Gell-Mann, A schematic model of baryons and mesons, Phys. Lett. 8 (1964) no. $3,214-215$.

[3] G. Zweig, An $\mathrm{SU}_{3}$ model for strong interaction symmetry and its breaking, Tech. Rep. CERN-TH-412, CERN, Geneva, 1964.

[4] J. J. Aubert et al., Experimental Observation of a Heavy Particle J, Phys. Rev. Lett. 33 (Dec, 1974) 1404-1406.

[5] J. E. Augustin et al., Discovery of a Narrow Resonance in $e^{+} e^{-}$Annihilation, Phys. Rev. Lett. 33 (Dec, 1974) 1406-1408.

[6] S. W. Herb et al., Observation of a Dimuon Resonance at $9.5 \mathrm{GeV}$ in 400-GeV Proton-Nucleus Collisions, Phys. Rev. Lett. 39 (Aug, 1977) 252-255.

[7] S. Abachi et al., D0 Collaboration, Search for High Mass Top Quark Production in $p \bar{p}$ Collisions at $\sqrt{s}=1.8 \mathrm{TeV}$, Phys. Rev. Lett. 74 (Mar, 1995) 2422-2426.

[8] F. Abe et al., CDF Collaboration, Observation of Top Quark Production in $\bar{p} p$ Collisions with the Collider Detector at Fermilab, Phys. Rev. Lett. 74 (Apr, 1995) 2626-2631.

[9] Super-Kamiokande Collaboration Collaboration, Y. Fukuda et al., Evidence for Oscillation of Atmospheric Neutrinos, Phys. Rev. Lett. 81 (Aug, 1998) $1562-1567$.

[10] Particle Data Group Collaboration, K. A. Olive et al., Review of Particle Physics, Chin. Phys. C38 (2014) 090001. 


\section{BIBLIOGRAPHY}

[11] E. Majorana, Teoria simmetrica dellelettrone e del positrone, Il Nuovo Cimento (1924-1942) 14 (1937) no. 4, 171-184.

[12] F. Englert and R. Brout, Broken Symmetry and the Mass of Gauge Vector Mesons, Phys. Rev. Lett. 13 (Aug, 1964) 321-323.

[13] P. W. Higgs, Broken Symmetries and the Masses of Gauge Bosons, Phys. Rev. Lett. 13 (Oct, 1964) 508-509.

[14] G. S. Guralnik, C. R. Hagen, and T. W. B. Kibble, Global Conservation Laws and Massless Particles, Phys. Rev. Lett. 13 (Nov, 1964) 585-587.

[15] ATLAS Collaboration, Observation of a new particle in the search for the Standard Model Higgs boson with the ATLAS detector at the LHC, Phys. Lett. B 716 (2012) no. 1, 1-29.

[16] CMS Collaboration, Observation of a new boson at a mass of $125 \mathrm{GeV}$ with the CMS experiment at the LHC, Phys. Lett. B 716 (2012) no. 1, 30-61.

[17] ATLAS Collaboration, Measurements of Higgs boson production and couplings in diboson final states with the ATLAS detector at the LHC, Phys. Lett. B 726 (2013) no. 1-3, 88-119.

[18] ATLAS Collaboration, Evidence for the spin-0 nature of the Higgs boson using ATLAS data, Phys. Lett. B 726 (2013) no. 1-3, 120-144.

[19] ATLAS Collaboration, Evidence for the Higgs-boson Yukawa coupling to tau leptons with the ATLAS detector, JHEP 2015 (2015) no. 4,.

[20] CMS Collaboration, Precise determination of the mass of the Higgs boson and tests of compatibility of its couplings with the standard model predictions using proton collisions at 7 and 8 TeV, EPJ C 75 (2015) no. 5, .

[21] CMS Collaboration, Constraints on the spin-parity and anomalous $H V V$ couplings of the Higgs boson in proton collisions at 7 and $8 \mathrm{TeV}$, Phys. Rev. D 92 (Jul, 2015) 012004.

[22] V. C. Rubin and W. K. Ford Jr, Rotation of the andromeda nebula from a spectroscopic survey of emission regions, ApJ 159 (1970) 379.

[23] D. Clowe et al., A Direct Empirical Proof of the Existence of Dark Matter, ApJL 648 (2006) no. 2, L109.

[24] A. Einstein, Über den Einfluß der Schwerkraft auf die Ausbreitung des Lichtes, Ann. Phys. 340 (1911) no. 10, 898-908.

[25] Planck Collaboration, P. A. R. Ade et al., Planck 2013 results. XVI. Cosmological parameters, Astron. Astrophys. 571 (2014) A16. 
[26] S. P. Martin, A Supersymmetry primer, ch. 1, pp. 1-98. World Scientific, 2011.

[27] ATLAS Collaboration, Search for the Standard Model Higgs boson produced in

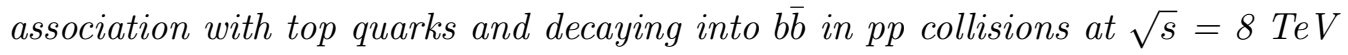
with the ATLAS detector, Eur. Phys. J. C75 (2015) no. 7, 349.

[28] LHC Higgs Cross Section Working Group, S. Dittmaier, C. Mariotti, G. Passarino, and R. Tanaka (Eds.), Handbook of LHC Higgs Cross Sections: 1. Inclusive Observables, CERN-2011-002 (CERN, Geneva, 2011) .

[29] ATLAS Collaboration, Search for flavour-changing neutral current top quark decays $t \rightarrow H q$ in pp collisions at $\sqrt{s}=8$ TeV with the ATLAS detector, Tech. Rep. CERN-PH-EP-2015-229. arXiv:1509.06047, CERN, Geneva, Sep, 2015.

[30] ATLAS Collaboration, The ATLAS experiment at the CERN large hadron collider, JINST 3 (2008) no. 08, S08003.

[31] ATLAS Collaboration, ATLAS Phase-II Upgrade Scoping Document, Tech. Rep. CERN-LHCC-2015-020. LHCC-G-166, CERN, Geneva, Sep, 2015.

[32] ALICE Collaboration, The ALICE experiment at the CERN LHC, JINST 3 (2008) no. 08, S08002.

[33] CMS Collaboration, The CMS experiment at the CERN LHC, JINST 3 (2008) no. 08 , S08004.

[34] LHCb Collaboration, The LHCb detector at the LHC, JINST 3 (2008) no. 08, S08005.

[35] L. Evans and P. Bryant, LHC machine, JINST 3 (2008) no. 08, S08001.

[36] https :

//twiki.cern.ch/twiki/bin/view/AtlasPublic/LuminosityPublicResults. retrieved on 21.08.2015.

[37] J. R. Pater, The ATLAS SemiConductor Tracker operation and performance, JINST 7 (2012) no. 04, C04001.

[38] V. Cindro et al., The ATLAS Beam Conditions Monitor, JINST 3 (2008) no. 02, P02004.

[39] M. Cerv, The ATLAS Diamond Beam Monitor, JINST 9 (2014) no. 02, C02026.

[40] http://hilumilhc.web.cern.ch/. retrieved on 17.09.2015.

[41] W. R. Leo, Techniques for Nuclear and Particle Physics Experiments: A How-To Approach. Springer Science \& Business Media, 1994.

[42] G. Lutz, Semiconductor radiation detectors. Springer, 1999. 


\section{BIBLIOGRAPHY}

[43] A. Holleman, E. Wiberg, and N. Wiberg, Lehrbuch der anorganischen Chemie. de Gruyter, 1995.

[44] H. Spieler, Semiconductor detector systems, vol. 12. Oxford University Press, 2005.

[45] W. Shockley, Currents to Conductors Induced by a Moving Point Charge, J. Appl. Phys. 9 (1938) no. 10, 635-636.

[46] S. Ramo, Currents Induced by Electron Motion, Proceedings of the IRE 27 (Sept, 1939) 584-585.

[47] G. Aad et al., ATLAS pixel detector electronics and sensors, JINST 3 (2008) no. 07, P07007.

[48] M. Capeans et al., ATLAS Insertable B-Layer Technical Design Report, Tech. Rep. CERN-LHCC-2010-013. ATLAS-TDR-19, CERN, Geneva, Sep, 2010.

[49] ATLAS Collaboration, Expected Performance of the ATLAS Experiment Detector, Trigger and Physics, arXiv:0901.0512 [hep-ex].

[50] R. Wunstorf, Systematische Untersuchungen zur Strahlenresistenz von Silizium-Detektoren für die Verwendung in Hochenergiephysik-Experimenten. PhD thesis, Universität Hamburg, 1992.

[51] M. Alam et al., The ATLAS silicon pixel sensors, Nucl. Instrum. Meth. A 456 (2001) no. 3, 217-232.

[52] M. Benoit, A. Lounis, and N. Dinu, Simulation of guard ring influence on the performance of ATLAS pixel detectors for inner layer replacement, JINST 4 (2009) P03025.

[53] C. Goessling et al., Evaluation of the breakdown behaviour of ATLAS silicon pixel sensors after partial guard-ring removal, Nucl. Instrum. Meth. A 624 (2010) no. 2, 410-413. New Developments in Radiation Detectors: Proceedings of the 11th European Symposium on Semiconductor Detectors.

[54] ATLAS IBL collaboration, Prototype ATLAS IBL modules using the FE-I4A front-end readout chip, JINST 7 (2012) no. 11, P11010.

[55] H. Briehl, Chemie der Werkstoffe. Springer Vieweg, 2014.

[56] E. Kasper and K. Lyutovich, eds., Properties of Silicon Germanium and SiGe: Carbon. IET, 1999.

[57] https://commons.wikimedia.org/wiki/File:Sp3-Orbital.svg. retrieved on 17.11.2015. 
[58] H. Pernegger, High mobility diamonds and particle detectors, Phys. Status Solidi A 203 (2006) no. 13, 3299-3314.

[59] H. Kagan, private communication, .

[60] Z. Li and H. Kraner, Modeling and simulation of charge collection properties for neutron irradiated silicon detectors, in Advanced technology and particle physics: Proceedings of the Third International Conference on Advanced Technology and Particle Physics, Como, Italy, 22-26 June 1992. 1993.

[61] H. Liander and E. Lundblad, Artificial diamonds, Asea Journal 28 (1955) no. 5-6, 97-98.

[62] A. D. Kiffler, Synthesis of diamond from carbon monoxide, tech. rep., Tonowanda Laboraties, Linde Air Products Co., 1956.

[63] I. Friel, Optical Engineering of Diamond, ch. 2. Optical Quality Diamond Grown by Chemical Vapor Deposition, pp. 35-69. Wiley-VCH Verlag GmbH \& Co. KGaA, 2013.

[64] R. S. Balmer et al., Chemical vapour deposition synthetic diamond: materials, technology and applications, J. Phys. Condens. Matter 21 (2009) no. 36, 364221.

[65] K. L. Choy, Chemical vapour deposition of coatings, Progress in Materials Science 48 (2003) no. 2, 57-170.

[66] M. Moll, E. Fretwurst, and G. Lindstrm, Leakage current of hadron irradiated silicon detectors material dependence, Nucl. Instrum. Meth. A 426 (1999) no. 1, $87-93$.

[67] W. de Boer et al., Radiation hardness of diamond and silicon sensors compared, Phys. Status Solidi A 204 (2007) no. 9, 3004-3010.

[68] E. Borchi and M. Bruzzi, Radiation damage in silicon detectors, La Rivista del Nuovo Cimento (1978-1999) 17 (1994) no. 11, 1-63.

[69] J.-W. Tsung, Diamond and Silicon Pixel Detectors in High Radiation Environments. PhD thesis, Universität Bonn, BONN-IR-2012-05, 2012.

[70] S. Parker, C. Kenney, and J. Segal, 3D A proposed new architecture for solid-state radiation detectors, Nucl. Instrum. Meth. A 395 (1997) no. 3, 328-343. Proceedings of the Third International Workshop on Semiconductor Pixel Detectors for Particles and X-rays.

[71] C. D. Via et al., 3D silicon sensors: Design, large area production and quality assurance for the ATLAS IBL pixel detector upgrade, Nucl. Instrum. Meth. A 694 (2012) 321-330. 


\section{BIBLIOGRAPHY}

[72] G. S. Sandhu and W. K. Chu, Reactive ion etching of diamond, Appl. Phys. Lett. 55 (1989) no. 5, 437-438.

[73] F. Nikolajeff and M. Karlsson, Optical Engineering of Diamond, ch. 4. Refractive and Diffractive Diamond Optics, pp. 109-142. Wiley-VCH Verlag GmbH \& Co. KGaA, 2013.

[74] C. Lee, E. Gu, and M. Dawson, Micro-cylindrical and micro-ring lenses in CVD diamond, Diam. Relat. Mater. 16 (2007) no. 47, 944-948. Proceedings of Diamond 2006, the 17th European Conference on Diamond, Diamond-Like Materials, Carbon Nanotubes, Nitrides and Silicon Carbide - Diamond 2006.

[75] L. Ondi et al., Effective Extraction of Photoluminescence from a Diamond Layer with a Photonic Crystal, ACS Nano 5 (2011) no. 1, 346-350.

[76] S. Tomljenovic-Hanic et al., Optical Engineering of Diamond, ch. 10. Diamond-Based Optical Waveguides, Cavities, and Other Microstructures, pp. 311-351. Wiley-VCH Verlag GmbH \& Co. KGaA, 2013.

[77] T. Kononenko et al., Microstructuring of diamond bulk by IR femtosecond laser pulses, Appl. Phys. A 90 (2008) no. 4, 645-651.

[78] W. Leemans et al., The BErkeley Lab Laser Accelerator (BELLA): A $10 \mathrm{GeV}$ Laser Plasma Accelerator, in AIP Conference Proceedings, vol. 1299, pp. 3-11. 2010 .

[79] V. I. Konov, T. V. Kononenko, and V. V. Kononenko, Optical Engineering of Diamond, ch. 12. Laser Micro- and Nanoprocessing of Diamond Materials, pp. 385-443. Wiley-VCH Verlag GmbH \& Co. KGaA, 2013.

[80] V. Konov, Laser in micro and nanoprocessing of diamond materials, Laser Photon. Rev. 6 (2012) no. 6, 739-766.

[81] G. Mainfray and G. Manus, Multiphoton ionization of atoms, Rep. Prog. Phys. 54 (1991) no. 10, 1333.

[82] M. Göppert-Mayer, Über Elementarakte mit zwei Quantensprüngen, Ann. Phys. 401 (1931) no. 3, 273-294.

[83] L. Orlov Yu, Mineralogy of Diamond, Izd. Acad. of Sci. of the USSR, Moscow (1973) .

[84] Y. V. Butenko et al., Kinetics of the graphitization of dispersed diamonds at low temperatures, J. Appl. Phys. 88 (2000) no. 7, 4380-4388.

[85] V. Strekalov, Graphitization of diamond stimulated by electron-hole recombination, Appl. Phys. A 80 (2005) no. 5, 1061-1066.

[86] J. Ready, Effects of high-power laser radiation. Academic Press, 1971. 
[87] V. Strekalov et al., Early stages of laser graphitization of diamond, Appl. Phys. A 76 (2003) no. 4, 603-607.

[88] V. V. Kononenko et al., Laser-induced structure transformations of diamonds, Proc. SPIE 5121 (2003) 259-270.

[89] G. Gottstein, Physikalische Grundlagen der Materialkunde. Springer Berlin Heidelberg, 2007.

[90] T. R. Anthony et al., Thermal diffusivity of isotopically enriched ${ }^{12} \mathrm{C}$ diamond, Phys. Rev. B 42 (Jul, 1990) 1104-1111.

[91] M. Neff et al., Femtosecond laser writing of buried graphitic structures in bulk diamond, Appl. Phys. A 97 (2009) no. 3, 543-547.

[92] T. Kononenko et al., Three-dimensional laser writing in diamond bulk, Diam. Relat. Mater. 20 (2011) no. 2, 264-268.

[93] T. Kononenko, A. Khomich, and V. Konov, Peculiarities of laser-induced material transformation inside diamond bulk, Diam. Relat. Mater. 37 (2013) $50-54$.

[94] F. Bachmair et al., A 3D diamond detector for particle tracking, Nucl. Instrum. Meth. A 786 (2015) 97-104.

[95] S. A. Campbell, The science and engineering of microelectronic fabrication. Oxford University Press, 2001.

[96] T. Fricke-Begemann, private communication, .

[97] H. C. Beck, Charakterisierung von Graphitelektroden in Diamant, Bachelor's thesis, Universität Göttingen, 2013.

[98] J. Kollmeier, Wachstum von Graphitsäulen im Diamant mittels Femtosekundenlaser, Bachelor's thesis, Universität Göttingen, 2013.

[99] D. M. Mattox, Handbook of Physical Vapor Deposition (PVD) Processing. Elsevier Inc., 2010.

[100] W. A. Harrison, Electronic Structure and the Properties of Solids: The Physics of the Chemical Bond. Dover Publications, Inc., 1989.

[101] J. W. Anthony et al., eds., Handbook of Mineralogy. Mineralogical Society of America, 2001.

[102] L. Föppel and E. Mönch, Praktische Spannungsoptik. Springer-Verlag, 1959.

[103] R. Mildren, Optical Engineering of Diamond, ch. 1. Intrinsic Optical Properties of Diamond, pp. 1-34. Wiley-VCH Verlag GmbH \& Co. KGaA, 2013. 


\section{BIBLIOGRAPHY}

[104] C. V. Raman, A new radiation, Indian J. Phys. 2 (1928) 387-398.

[105] C. Kittel, Einführung in die Festkörperphysik. Oldenbourg, 2005.

[106] S. A. Solin and A. K. Ramdas, Raman Spectrum of Diamond, Phys. Rev. B 1 (Feb, 1970) 1687-1698.

[107] R. Al-Jishi and G. Dresselhaus, Lattice-dynamical model for graphite, Phys. Rev. B 26 (Oct, 1982) 4514-4522.

[108] R. J. Nemanich and S. A. Solin, First- and second-order Raman scattering from finite-size crystals of graphite, Phys. Rev. B 20 (Jul, 1979) 392-401.

[109] A. C. Ferrari and J. Robertson, Interpretation of Raman spectra of disordered and amorphous carbon, Phys. Rev. B 61 (May, 2000) 14095-14107.

[110] A. M. Bonnot, Raman microspectroscopy of diamond crystals and thin films prepared by hot-filament-assisted chemical vapor deposition, Phys. Rev. B 41 (Mar, 1990) 6040-6049.

[111] G. Cowan, Statistical data analysis. Oxford University Press, 1998.

[112] S. R. Sails et al., Monitoring the quality of diamond films using Raman spectra excited at 514.5 nm and $633 \mathrm{~nm}$, Diam. Relat. Mater. 5 (1996) no. 68, 589-591. Proceedings of the 6th European Conference on Diamond, Diamond-like and Related Materials Part 2.

[113] P. K. Chu and L. Li, Characterization of amorphous and nanocrystalline carbon films, Mater. Chem. Phys. 96 (2006) no. 23, 253-277.

[114] F. C. Tai et al., Multipeak fitting analysis of Raman spectra on DLCH film, J. Raman Spectrosc. 40 (2009) no. 8, 1055-1059.

[115] F. Tuinstra and J. L. Koenig, Raman Spectrum of Graphite, J. Chem. Phys. 53 (1970) no. 3, 1126-1130.

[116] M. J. Matthews et al., Origin of dispersive effects of the Raman D band in carbon materials, Phys. Rev. B 59 (Mar, 1999) R6585-R6588.

[117] https://www. asylumresearch.com/Probe/AC160TS-R3, Olympus. retrieved on 01.12.2015.

[118] S. N. Magonov and M.-H. Whangbo, Surface analysis with STM and AFM. VCH, 1996. 
Appendices 

APPENDIX $\mathrm{A}$

Photos of Phase Change in polycrystalline Diamond A.1. Goe-pCVD-01 


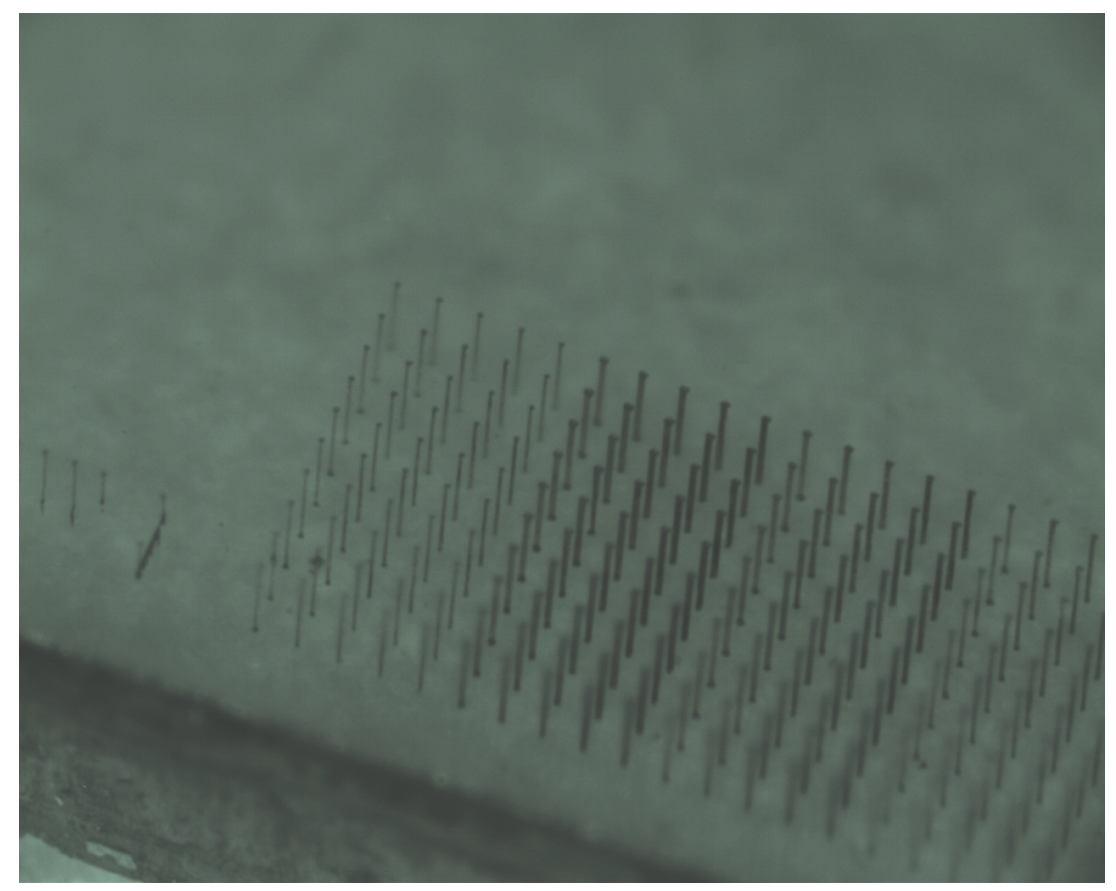

(a)

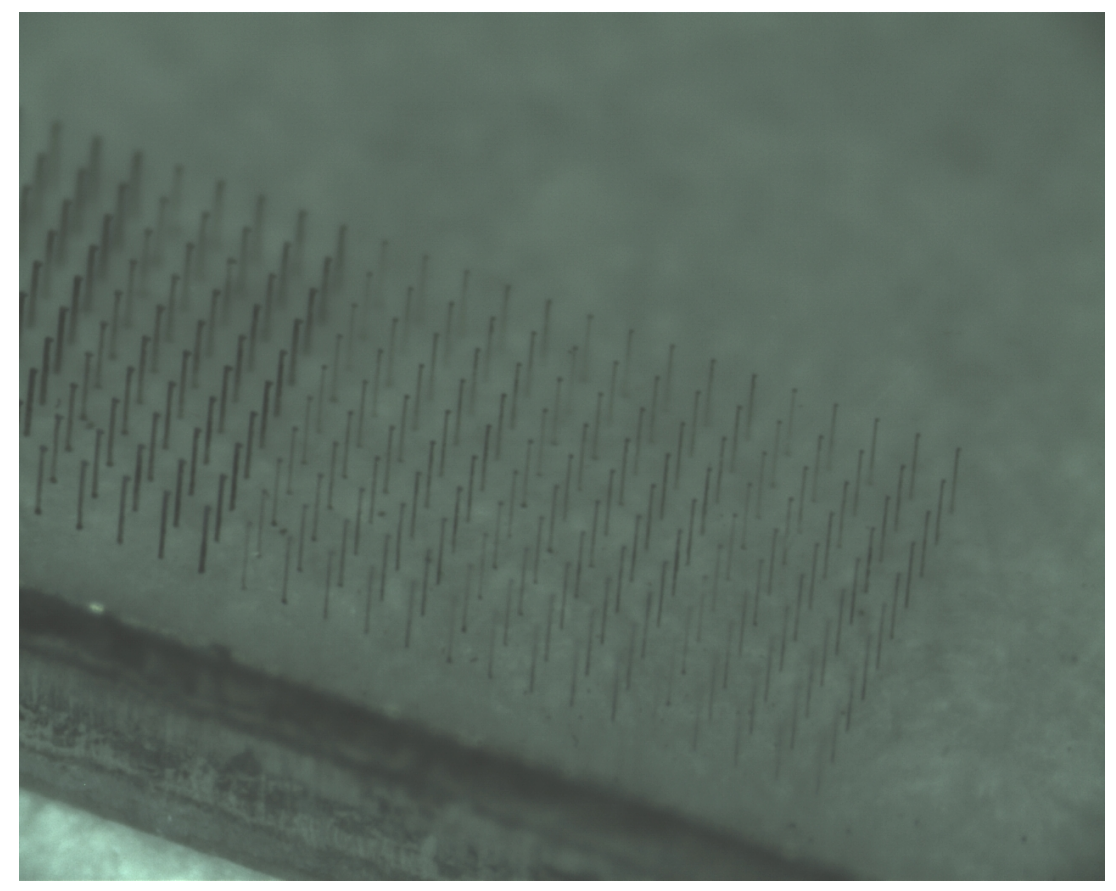

(b)

Figure A.1.: Channels produced in the second batch in Goe-pCVD-01. The growth parameters for the channels are a laser power of 40,45, 50,60, 80,100,120 mW and a sample velocity of $5,10,20,40,70 \mu \mathrm{m} / \mathrm{s}$. On the left side of figure (a), channels produced in the first batch can be seen. The diamond is slightly tilted with the exit side being on top. 


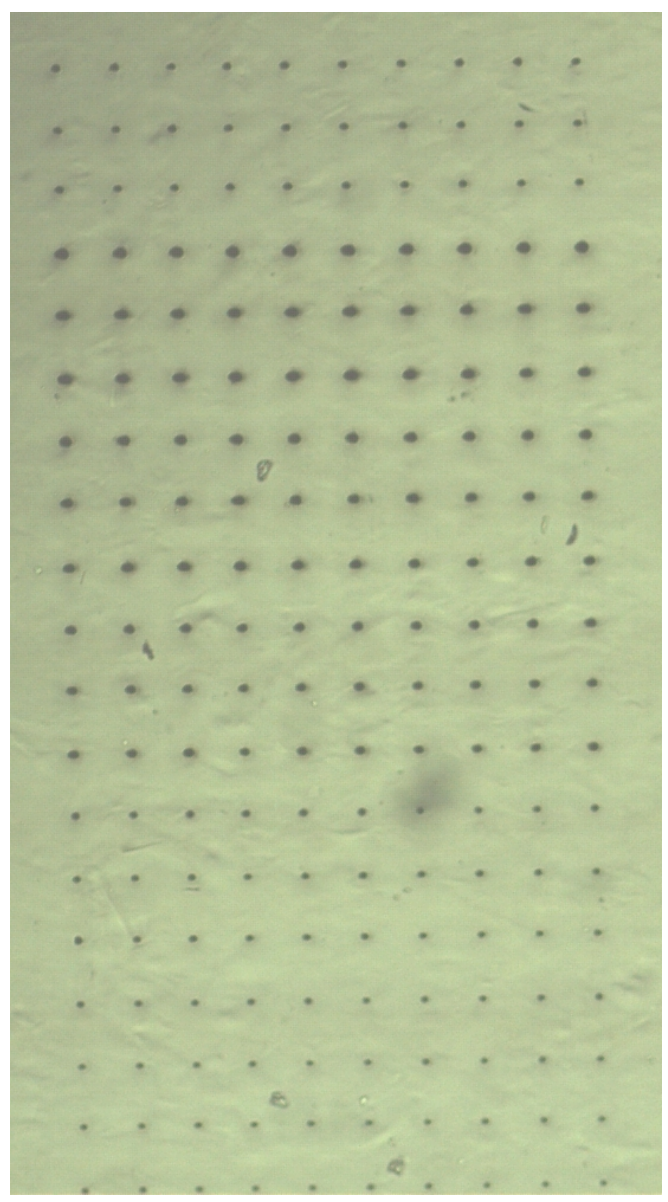

(a)

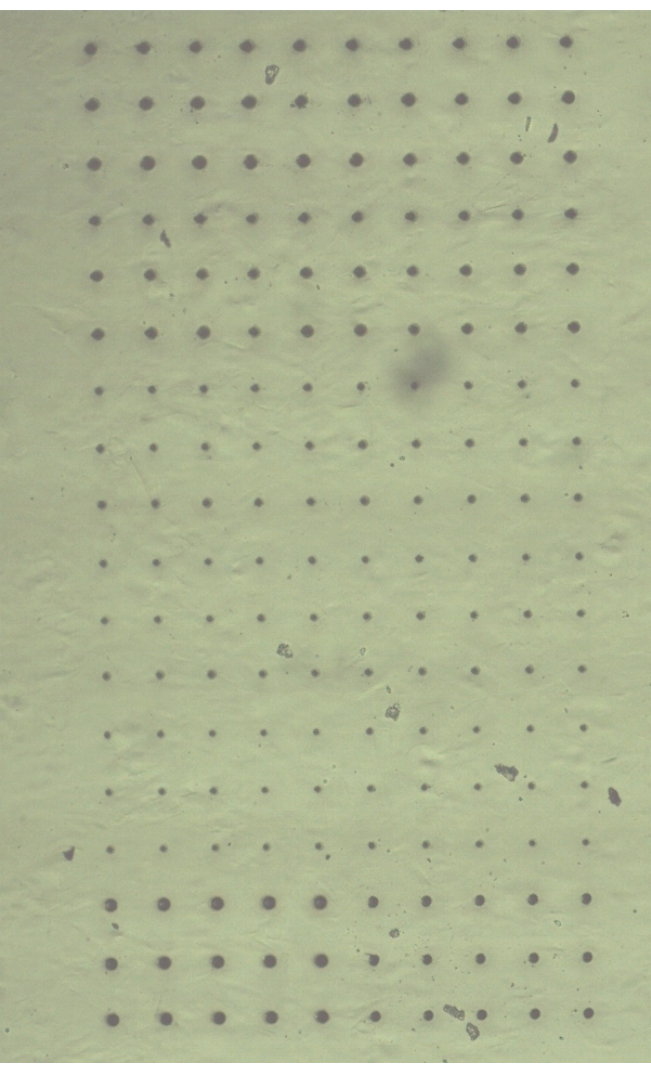

(b)

Figure A.2.: Photos of the exit side of channels produced in the third batch in GoepCVD-01. The same laser powers as for the first batch were used and sample velocities of $20,40,70 \mu \mathrm{m} / \mathrm{s}$. The lower three rows in figure (b) were produced under a nitrogen atmosphere. 


\section{A.2. Goe-pCVD-02}

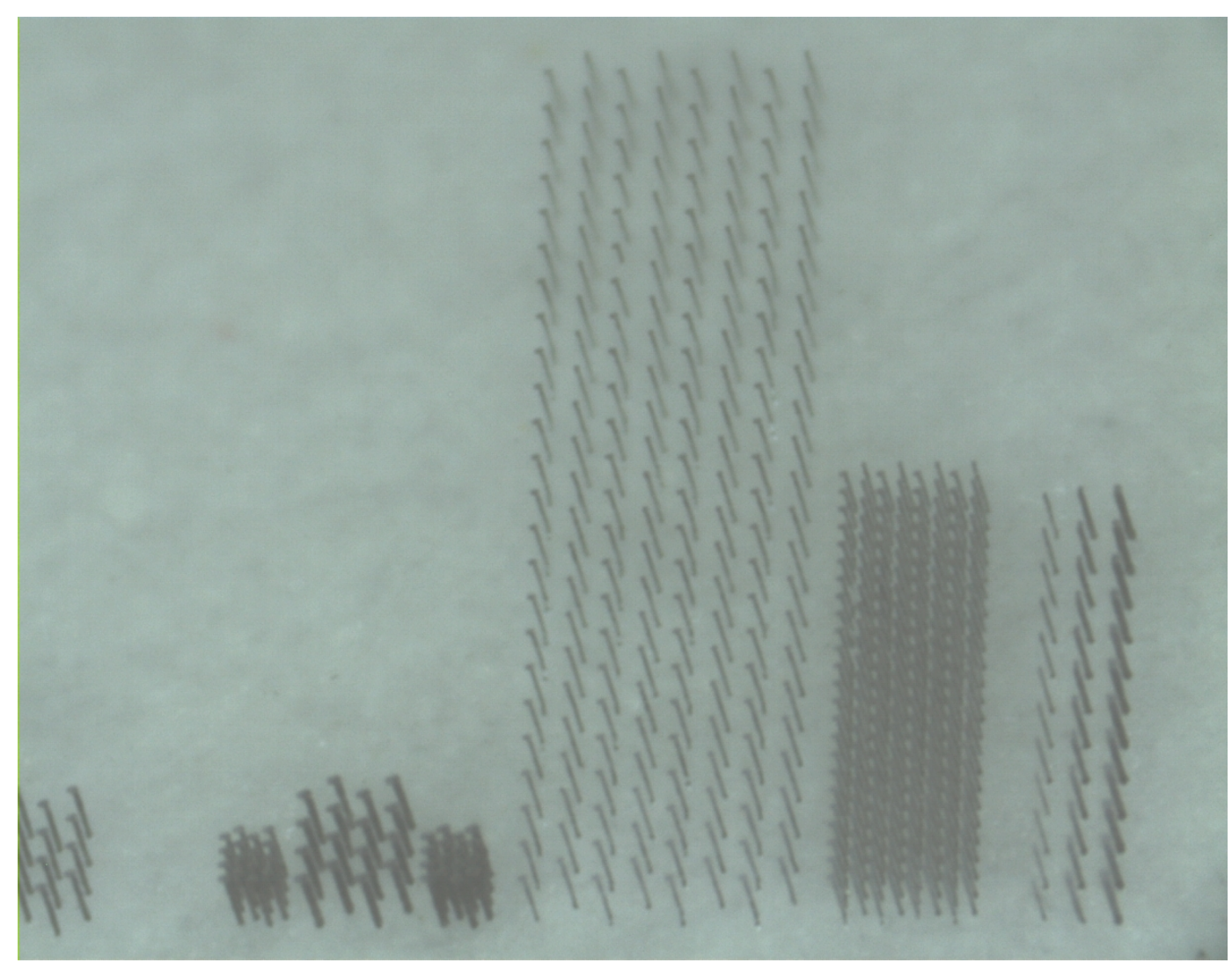

Figure A.3.: Photo of the channels produced in Goe-pCVD-02. In the centre the large grid with a spacing of $50 \mu \mathrm{m}$ can be seen. Right next to it a grid with an equal number of channels but with a spacing of $25 \mu \mathrm{m}$. The remaining channels on the left can be seen in figure 6.6. 


\section{A.3. Goe-pCVD-03}

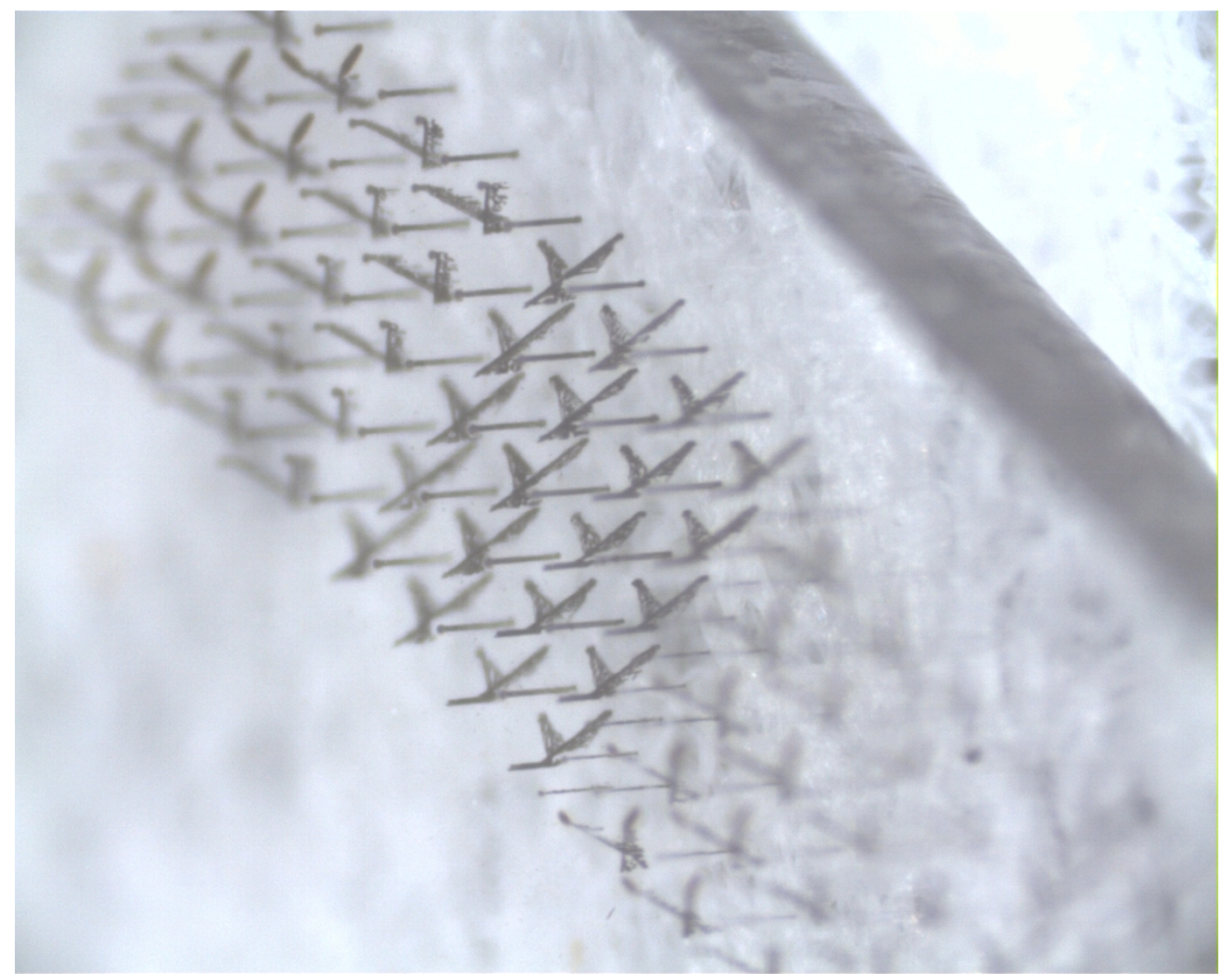

Figure A.4.: Photo of triangular channels with contacts on the seed side. The channels were produced with a laser power of $100 \mathrm{~mW}$. 

APPENDIX B

Raman Spectra of Phase Changed Material

\section{B.1. Batch 2 of the detector grade pCVD Diamond Sample}

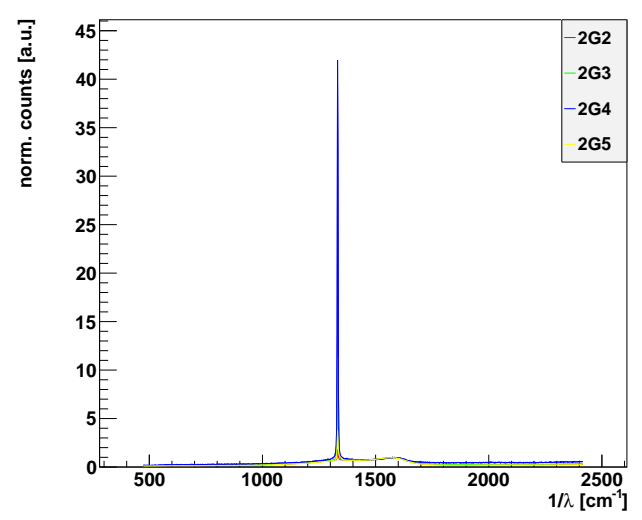

(a)

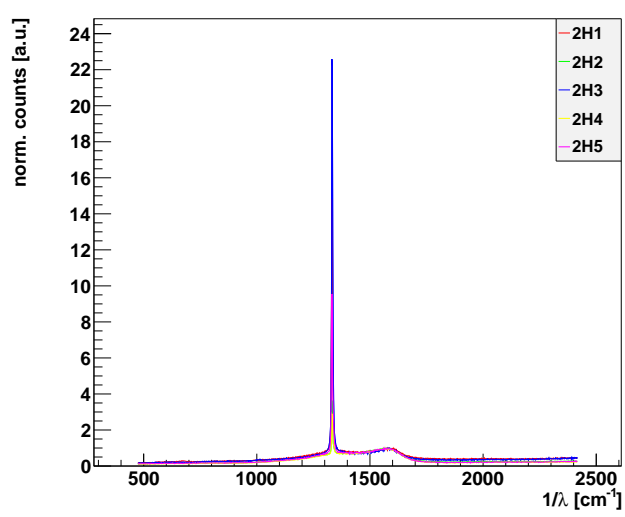

(b)

Figure B.1.: Raman spectra of the seed side of channels produced with a laser power of $10 \mathrm{~mW}$ and a sample velocity of (a) $50 \mu \mathrm{m} / \mathrm{s}$ and (b) $200 \mu \mathrm{m} / \mathrm{s}$. 


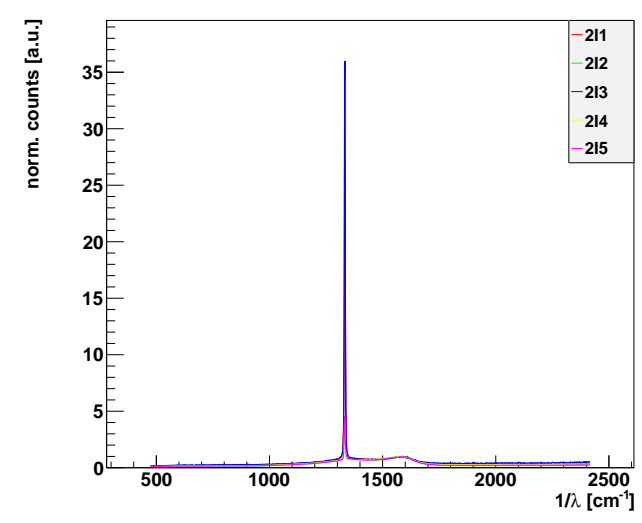

(a)

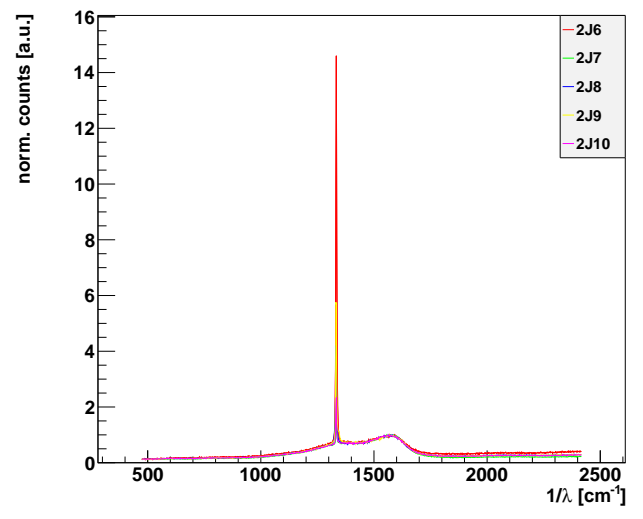

(b)

Figure B.2.: Raman spectra of the seed side of channels produced with a laser power of $10 \mathrm{~mW}$ and a sample velocity of (a) $2 \mathrm{~mm} / \mathrm{s}$ and (b) $10 \mathrm{~mm} / \mathrm{s}$.

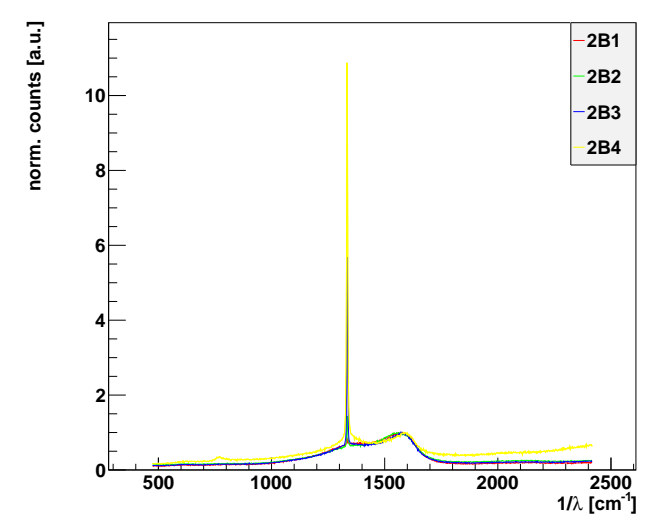

(a)

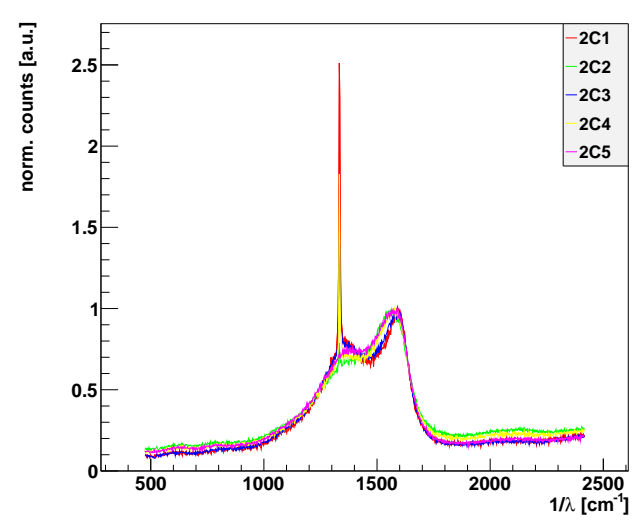

(b)

Figure B.3.: Raman spectra of the seed side of channels produced with a laser power of $25 \mathrm{~mW}$ and a sample velocity of (a) $50 \mu \mathrm{m} / \mathrm{s}$ and (b) $200 \mu \mathrm{m} / \mathrm{s}$. 


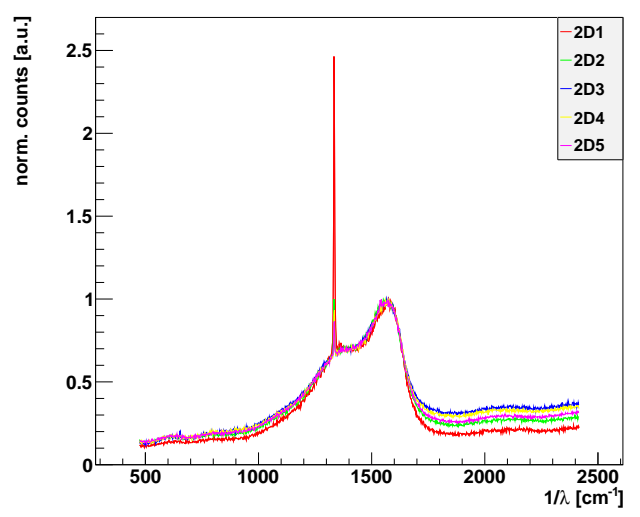

(a)

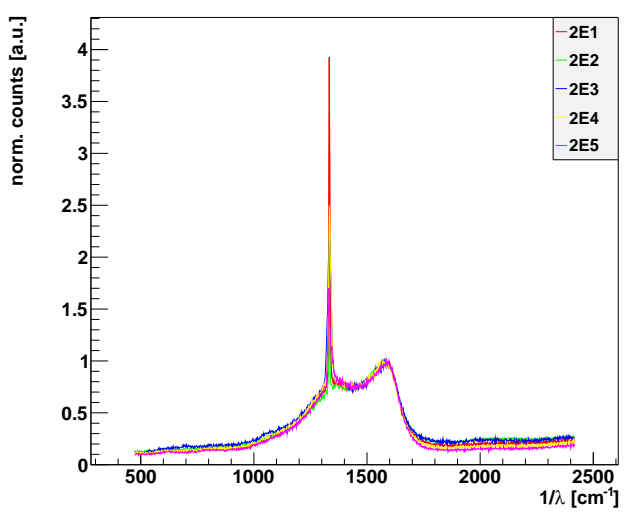

(b)

Figure B.4.: Raman spectra of the seed side of channels produced with a laser power of $25 \mathrm{~mW}$ and a sample velocity of (a) $2 \mathrm{~mm} / \mathrm{s}$ and (b) $10 \mathrm{~mm} / \mathrm{s}$.

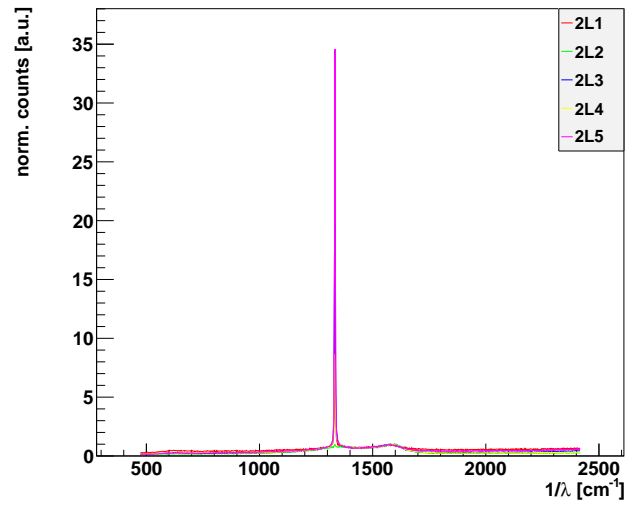

(a)

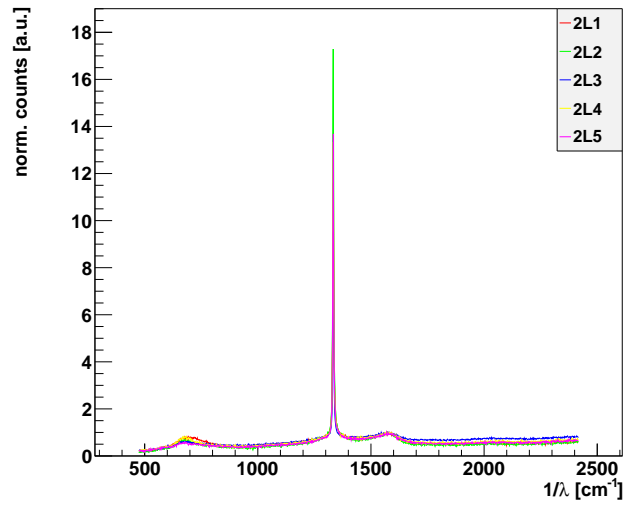

(b)

Figure B.5.: Raman spectra of channels produced with a laser power of $60 \mathrm{~mW}$ and a sample velocity of $50 \mu \mathrm{m} / \mathrm{s}$ for (a) the seed and (b) the exit side. 


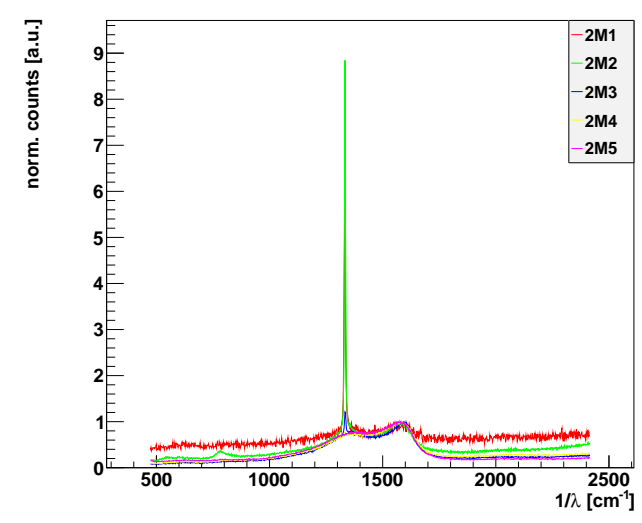

(a)

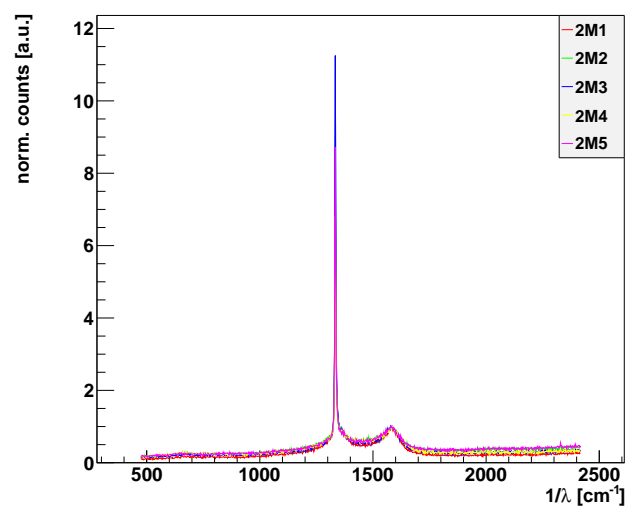

(b)

Figure B.6.: Raman spectra of channels produced with a laser power of $60 \mathrm{~mW}$ and a sample velocity of $200 \mu \mathrm{m} / \mathrm{s}$ for (a) the seed and (b) the exit side.

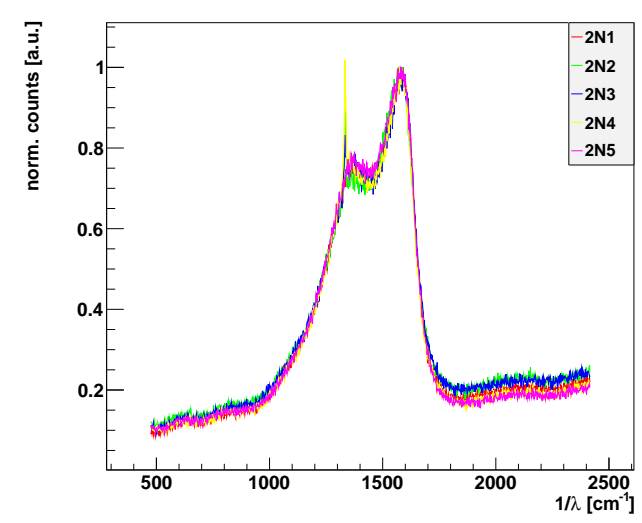

(a)

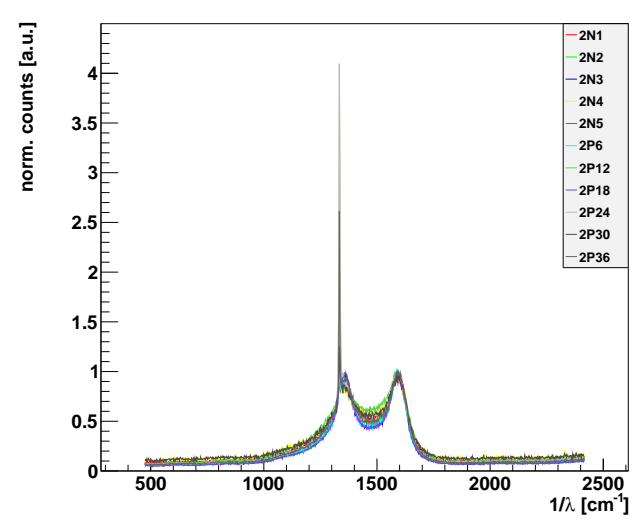

(b)

Figure B.7.: Raman spectra of channels produced with a laser power of $60 \mathrm{~mW}$ and a sample velocity of $2 \mathrm{~mm} / \mathrm{s}$ for (a) the seed and (b) the exit side. 


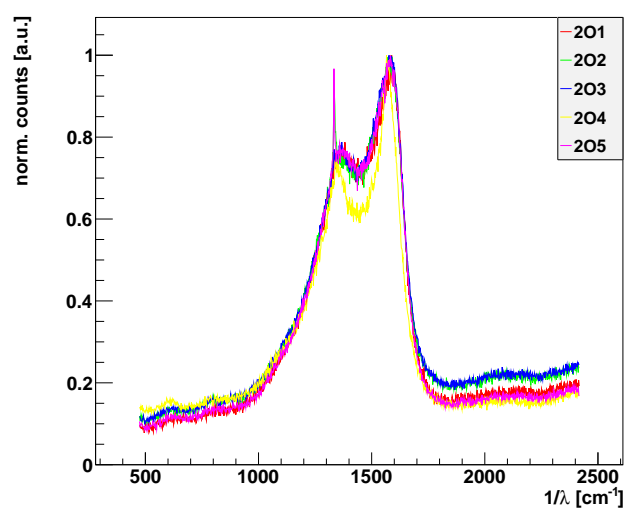

(a)

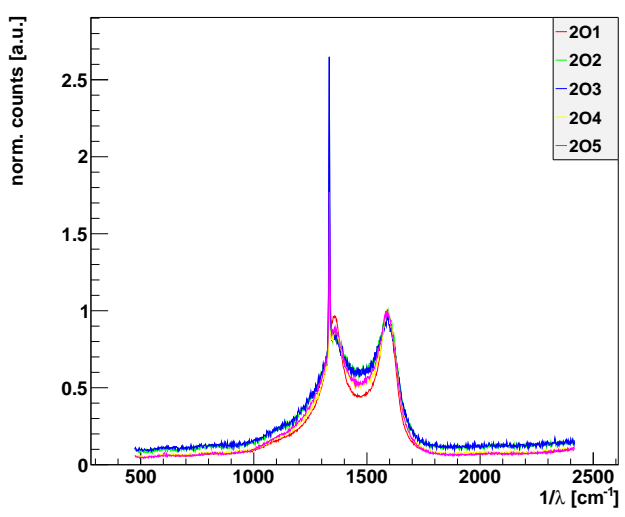

(b)

Figure B.8.: Raman spectra of channels produced with a laser power of $60 \mathrm{~mW}$ and a sample velocity of $10 \mathrm{~mm} / \mathrm{s}$ for (a) the seed and (b) the exit side.

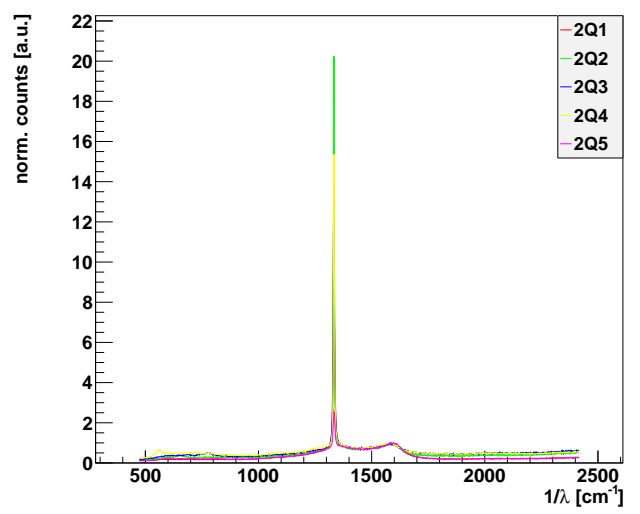

(a)

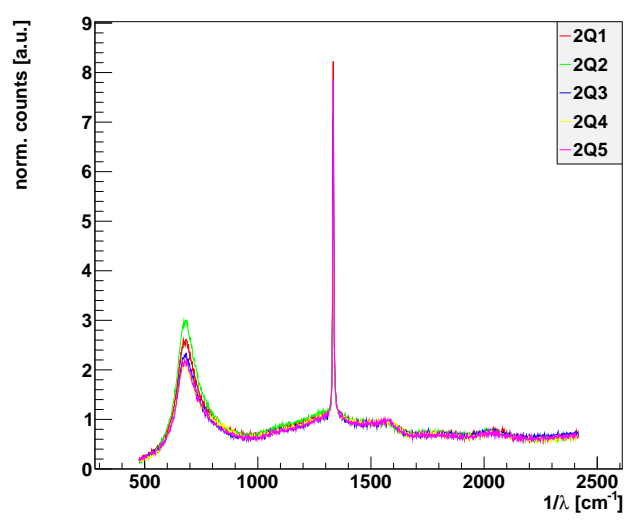

(b)

Figure B.9.: Raman spectra of channels produced with a laser power of $150 \mathrm{~mW}$ and a sample velocity of $50 \mu \mathrm{m} / \mathrm{s}$ for (a) the seed and (b) the exit side. 


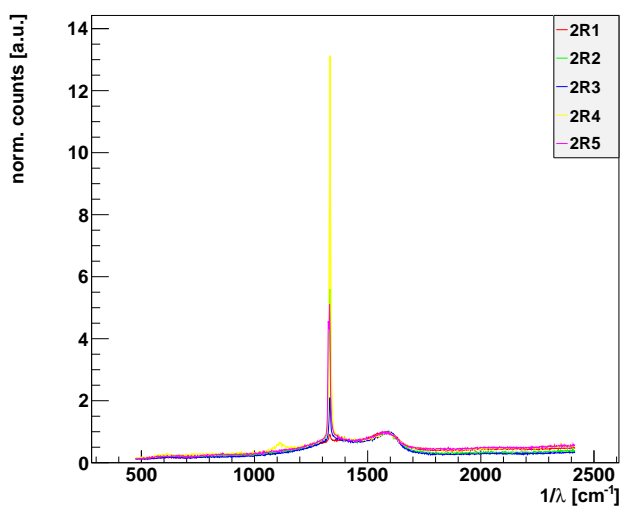

(a)

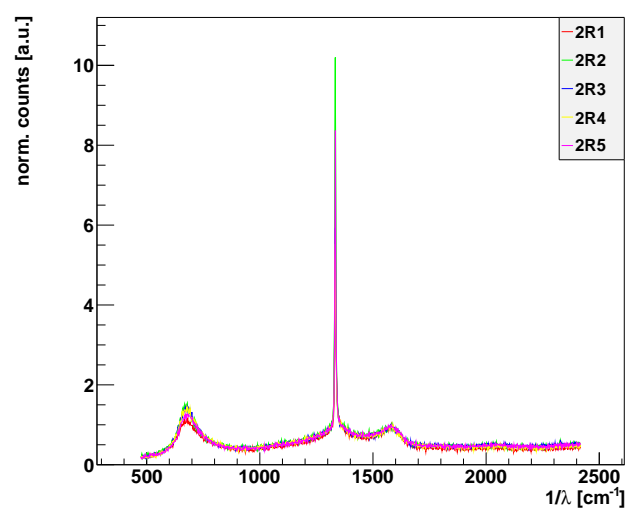

(b)

Figure B.10.: Raman spectra of channels produced with a laser power of $150 \mathrm{~mW}$ and a sample velocity of $200 \mu \mathrm{m} / \mathrm{s}$ for (a) the seed and (b) the exit side.

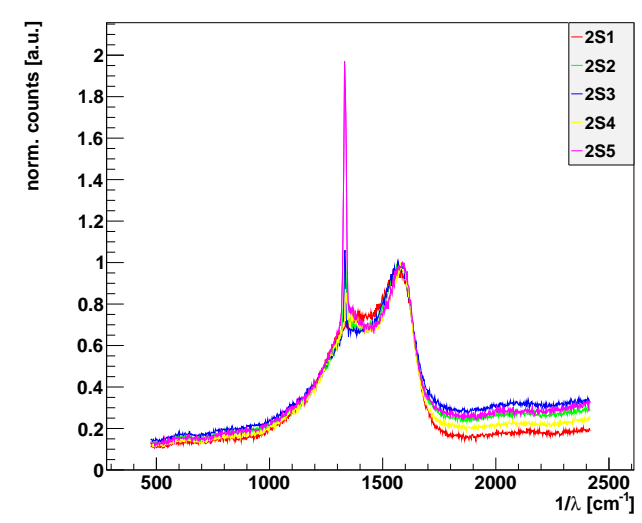

(a)

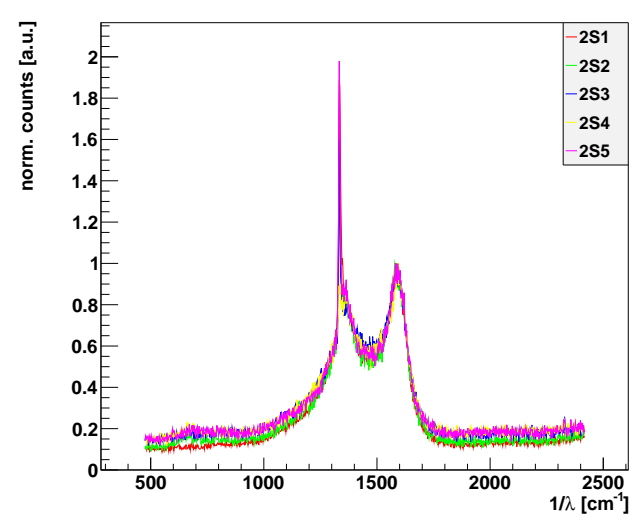

(b)

Figure B.11.: Raman spectra of channels produced with a laser power of $150 \mathrm{~mW}$ and a sample velocity of $2 \mathrm{~mm} / \mathrm{s}$ for (a) the seed and (b) the exit side. 


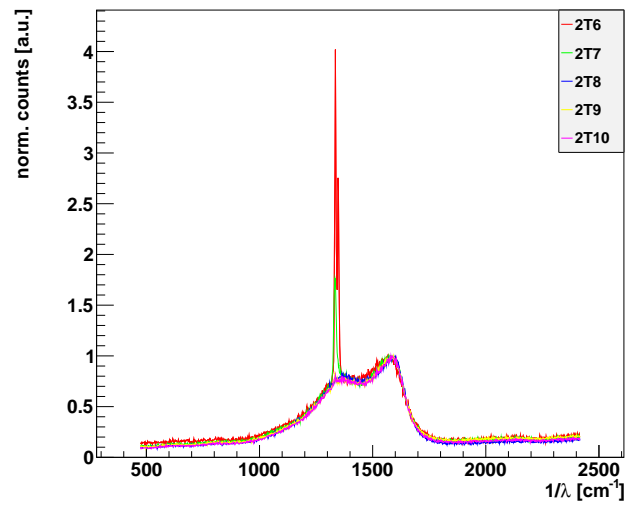

(a)

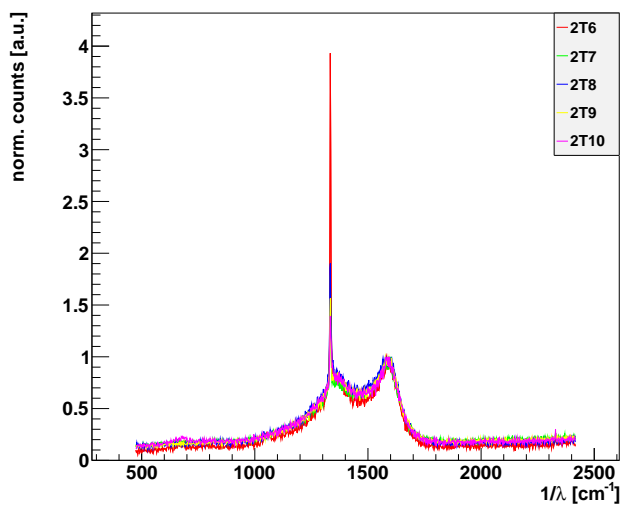

(b)

Figure B.12.: Raman spectra of channels produced with a laser power of $150 \mathrm{~mW}$ and a sample velocity of $10 \mathrm{~mm} / \mathrm{s}$ for (a) the seed and (b) the exit side. 


\section{B.2. Batch 3 of the detector grade pCVD Diamond Sample}

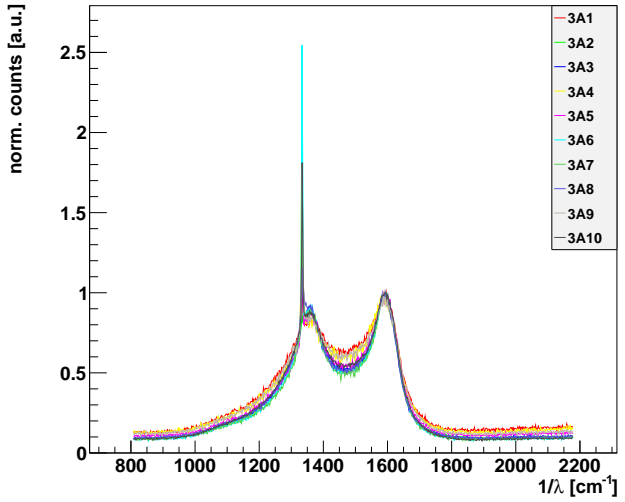

(a)

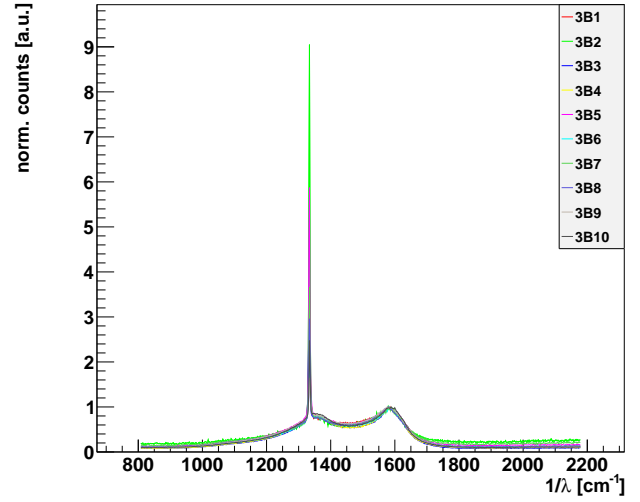

(b)

Figure B.13.: Raman spectra of channels produced with a laser power of $60 \mathrm{~mW}$ and a sample velocity of $2 \mathrm{~mm} / \mathrm{s}$ (a) without and (b) with reirradiation of the exit side.

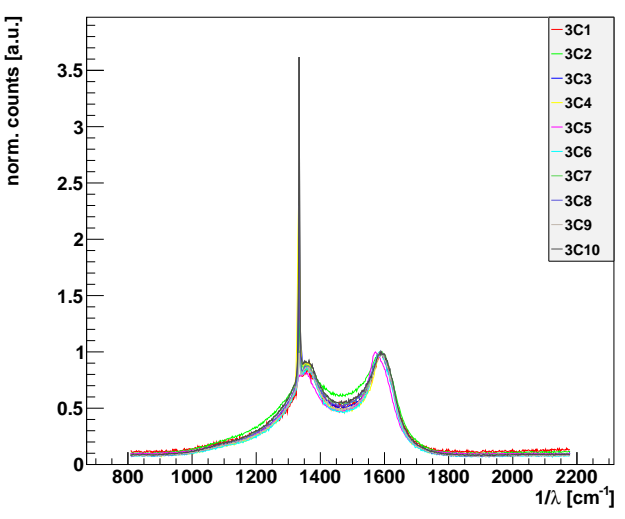

(a)

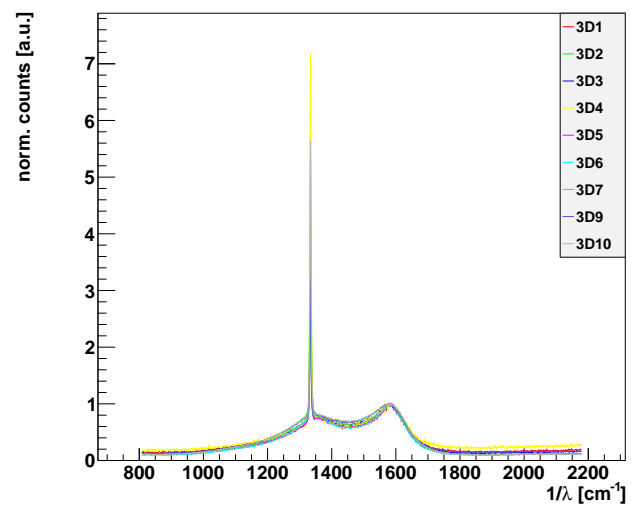

(b)

Figure B.14.: Raman spectra of channels produced with a laser power of $60 \mathrm{~mW}$ and a sample velocity of $10 \mathrm{~mm} / \mathrm{s}$ (a) without and (b) with reirradiation of the exit side. 


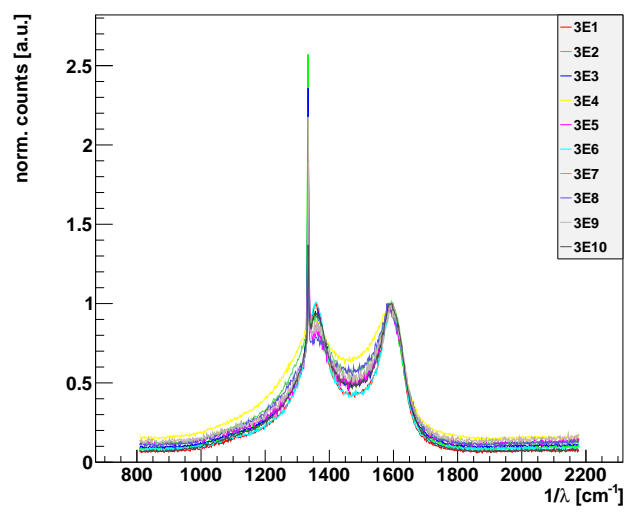

(a)

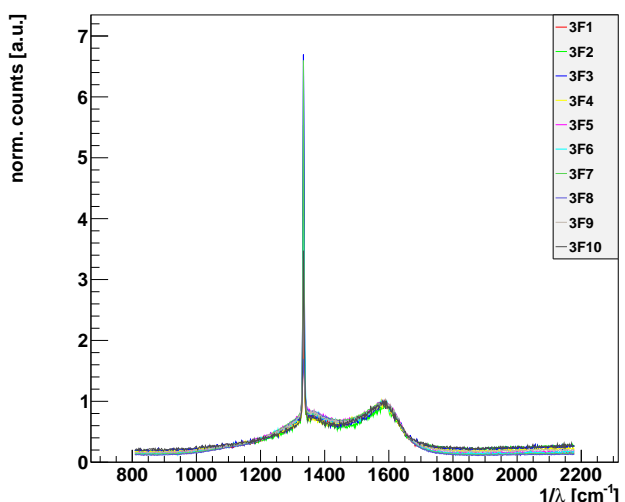

(b)

Figure B.15.: Raman spectra of channels produced with a laser power of $100 \mathrm{~mW}$ and a sample velocity of $2 \mathrm{~mm} / \mathrm{s}$ (a) without and (b) with reirradiation of the exit side.

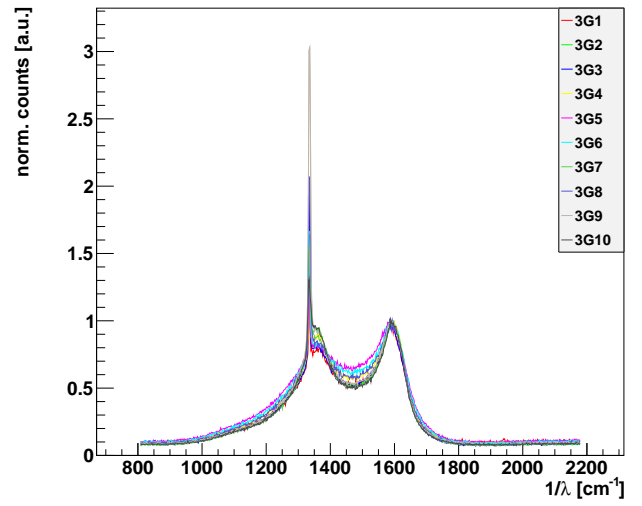

(a)

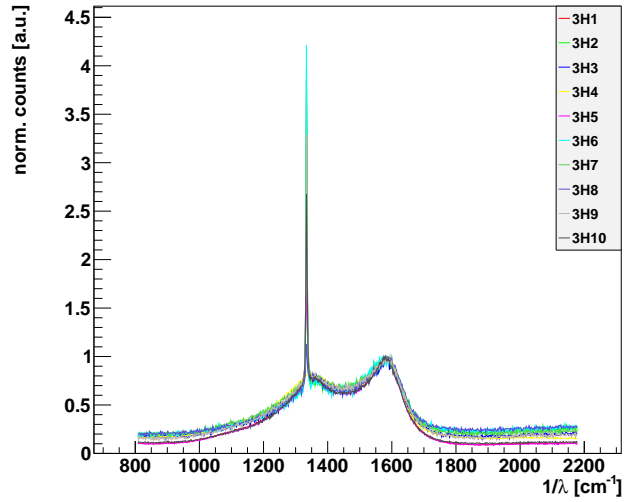

(b)

Figure B.16.: Raman spectra of channels produced with a laser power of $100 \mathrm{~mW}$ and a sample velocity of $10 \mathrm{~mm} / \mathrm{s}$ (a) without and (b) with reirradiation of the exit side. 


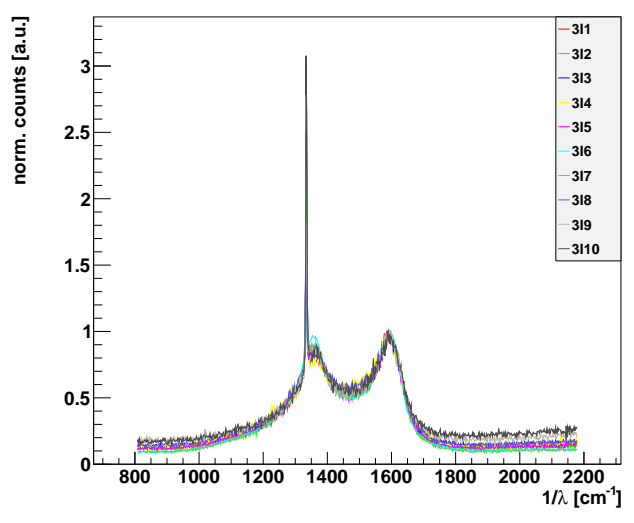

(a)

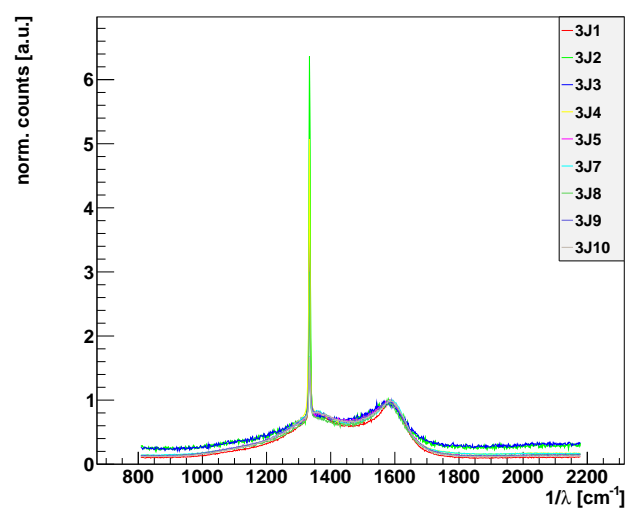

(b)

Figure B.17.: Raman spectra of channels produced with a laser power of $150 \mathrm{~mW}$ and a sample velocity of $2 \mathrm{~mm} / \mathrm{s}$ (a) without and (b) with reirradiation of the exit side.

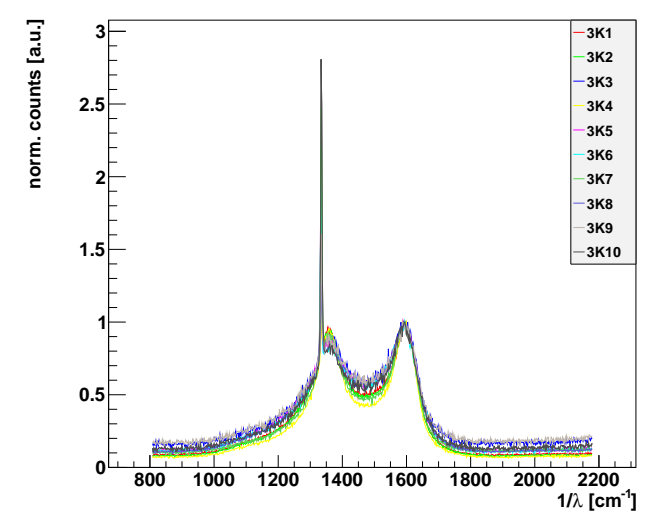

(a)

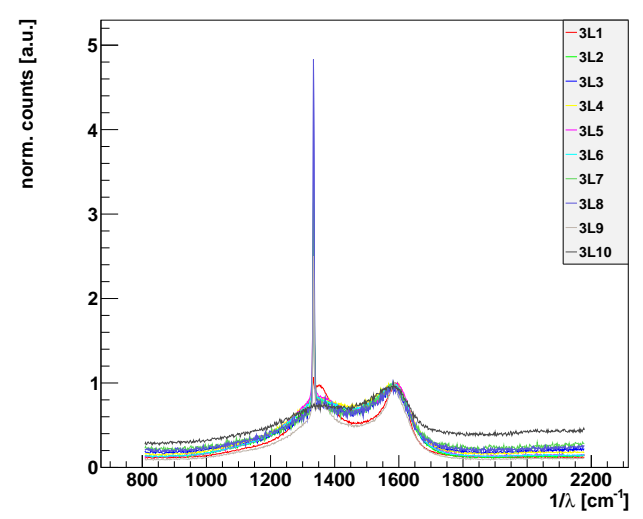

(b)

Figure B.18.: Raman spectra of channels produced with a laser power of $150 \mathrm{~mW}$ and a sample velocity of $10 \mathrm{~mm} / \mathrm{s}$ (a) without and (b) with reirradiation of the exit side. 